\title{
Detector Physics of Resistive Plate Chambers
}

\author{
Dissertation \\ zur Erlangung des Doktorgrades \\ der Naturwissenschaften
}

\begin{abstract}
vorgelegt beim Fachbereich Physik
der Johann Wolfgang Goethe-Universität

in Frankfurt am Main
\end{abstract}

\author{
durchgeführt am \\ Europäischen Kernforschungszentrum, \\ European Organization for Nuclear Research \\ (CERN) \\ in Genf, Schweiz
}

\section{von \\ Christian Lippmann aus Kronberg/Ts.}

Frankfurt am Main, 2003

(DF1) 
vom Fachbereich Physik der Johann Wolfgang Goethe-Universität als Dissertation angenommen.

Dekan: Prof. Dr. Horst Schmidt-Böcking

1. Gutachter: Prof. Dr. Reinhard Stock

2. Gutachter: Prof. Dr. Herbert Ströbele

3. Gutachter: Dr. Werner Riegler

Datum der Disputation: 


\section{Zusammenfassung}

Widerstandsplattenkammern (im englischen "Resistive Plate Chambers" oder abgekürzt "RPCs") sind Teilchendetektoren, die aus zwei parallelen planaren Elektroden bestehen, die einen gasgefüllten Spalt von wenigen hundert Micrometern bis zu einigen Millimetern umschließen. Wenigstens eine der beiden Elektroden besteht aus einem Material mit hohem Volumenwiderstand von $10^{7}$ bis $10^{12} \Omega \mathrm{cm}$. Die Vorteile dieser Technologie sind die gute Zeitauflösung (bis zu $50 \mathrm{ps)} \mathrm{bei} \mathrm{einer} \mathrm{guten} \mathrm{Nach-}$ weiseffizienz $(\approx 99 \%$ für mehrere kombinierte Zähler) und der einfache technische Aufbau. In den Hochenergiephysikexperimenten ATLAS und CMS, die derzeit am CERN in Genf aufgebaut werden, sollen im sogenannten Lawinenmodus betriebene RPCs als schnelle Auslösezähler (Trigger RPCs) auf Flächen von mehreren tausend Quadratmetern eingesetzt werden. Im Experiment ALICE am CERN finden Timing $R P C s$ für präzise Flugzeitmessungen auf einer Fläche von $176 \mathrm{~m}^{2}$ Anwendung.

RPCs wurden ursprünglich im Streamermodus betrieben, welcher die Anforderungen an die Ausleseelektronik und die Genauigkeit des Elektrodenabstandes vereinfacht. Um verbesserte Hochratenfestigkeit und verminderte Alterung der RPCs zu erlangen, wurde der Betrieb im Lawinenmodus populär. Diese Entwicklung wurde möglich durch die Einführung neuer Gasmischungen auf der Basis von $\mathrm{C}_{2} \mathrm{~F}_{4} \mathrm{H}_{2}$ mit geringen $\mathrm{SF}_{6}$-Beimischungen. Während Streamer schwer zu studieren sind, eröffnete der Lawinenmodus die Möglichkeit detaillierter Studien der physikalischen Prozesse in RPCs.

Trotz des intensiven Einsatzes der RPC Technologie sind einige experimentelle Ergebnisse noch nicht genau verstanden. Insbesondere hinsichtlich der Erklärung der guten Nachweiseffizienz der Timing RPCs mit ihrem kleinen Plattenabstand von 0.2 bis $0.3 \mathrm{~mm}$ kamen vielerlei Fragen auf. So steht der für die gute Nachweiseffizienz nötige hohe Wert für die Gasverstärkung in krassem Widerspruch zu den gemessenen niedrigen Ladungen um 1 pC. Es tut sich eine Diskrepanz auf, die sieben Größenordnungen erreichen kann. Deshalb wurde vorgeschlagen, die hohe Effizienz anhand von Begleitelektronen zu erklären, die vom Primärteilchen aus dem Detektorrahmen gelöst werden. Auf der anderen Seite könnte ein sehr starker Raumladungseffekt die Ladungen zu höheren Werten hin begrenzen. Der Begriff Raumladungseffekt beschreibt den dynamischen Prozess der Verzerrung des angelegten elektrischen Feldes durch die Ladungsträger in der Lawine. Um die großen Ladungen unterdrücken zu können, muss der Raumladungseffekt eine gewisse Stärke haben, und das angelegte elektrische Feld an den Positionen, an denen sich der Großteil der driftenden Elektronen in der Lawine befindet, stark erniedrigen. Dann muss aber das Feld an anderen Positionen durch den gleichen Effekt stark erhöht sein, was neue Fragen hervorruft. Viele Autoren lehnen die Möglichkeit ab, dass sich eine Lawine unter diesen extremen Umständen ausbreiten kann, ohne dass sie sich in einen Streamer umwandelt. 
Schließlich ist der Grund für die experimentell beobachtete Form der Ladungsspektren an RPCs nicht eindeutig geklärt. Man beobachtet einen Scheitelpunkt, der zu höheren Spannungen hin ausgeprägter wird. Die Statistik der Elektronenlawinen sagt jedoch ein monoton zu höheren Ladungen hin abfallendes Spektrum voraus.

In der vorliegenden Arbeit werden im Detail die physikalischen Prozesse beschrieben, die grundlegend für die Funktion und für das Verständnis von RPCs sind, beginnend bei der Primärionisation, über die Lawinenstatistik bis zur Signalinduktion und zur Ausleseelektronik. Die für die Primärionisation und die Lawinenpropagation wichtigen Gasparameter werden mit den bekannten Simulationsprogrammen HEED, MAGBOLTZ und IMONTE errechnet. Es werden Monte-Carlo Simulationsroutinen vorgestellt, die auf den beschriebenen Prozessen basieren. Ein einfaches eindimensionales Modell ohne Diffusionseffekte und ohne detaillierte Implementierung eines Raumladungseffektes ergibt Simulationsergebnisse für Effizienz und Zeitauflösung, die sehr nahe an Messungen liegen. Dieses Modell und die Ergebnisse wurden publiziert in [1]]. Den Beitrag der vorliegenden Arbeit bilden die Implementierung des Raumladungseffektes in dieses Modell und seine detaillierte Untersuchung.

Der Raumladungseffekt wird mit eingebunden, indem angenommen wird, dass die Lawinenladungen in Scheiben untergebracht sind, welche radiale Gaußförmige Ladungsverteilungen tragen, die von der transversalen Diffusion abhängen. Für die Berechnung des elektrischen Feldes der Raumladung wird eine analytische Lösung für das Potential einer Punktladung im Gasspalt einer RPC verwendet. Diese wurden in Zusammenarbeit mit der Technischen Universität Graz [2] erarbeitet und in [3, 4] publiziert. Schließlich werden mehrdimensionale Modelle präsentiert, die auch die Auswirkungen des Raumladungsfeldes in transversale Richtung implementieren. Insbesondere das 2-D Modell, welches Zylindersymmetrie der Lawinen annimmt und den Spalt in ein zweidimensionales Netz der longitudinalen und radialen Koordinaten einteilt, erlaubt die detaillierte und erkenntnisreiche Simulation einzelner Ladungslawinen.

Es wird gezeigt, dass die hohe RPC Effizienz tatsächlich durch eine hohen Dichte von Primärionisationszentren (etwa $9.5 / \mathrm{cm}$ ) und durch einen hohen effektiven Townsend-Koeffizienten (etwa $113 / \mathrm{mm}$ ) erklärt wird. Es ergibt sich, dass das Raumladungsfeld bei hoher Gasverstärkung die Größenordnung des angelegten Feldes erreicht, in longitudinaler wie in transversaler Richtung. Der Raumladungseffekt unterdrückt tatsächlich die großen Werte der Ladungen. Es wird gezeigt, dass die Form der simulierten Ladungsspektren sehr genau denen von gemessenen Spektren gleicht, und dass die mittlere Ladung der simulierten Spektren nahe an den gemessenen liegt. Außerdem wird gezeigt, dass RPCs in einem Raumladungsmodus betrieben werden, welcher sich über einen großen Bereich angelegter Spannungen erstreckt, im Gegensatz zu Drahtkammern.

Die Ergebnisse der vorliegenden Arbeit wurden zum Teil auf der 'RPC 2001'Konferenz [5] und auf der '2002 NSS/MIC'-Konferenz [6] vorgestellt und diskutiert. 


\begin{abstract}
Resistive Plate Chambers (RPCs) are gaseous parallel plate avalanche detectors that implement electrodes made from a material with a high volume resistivity between $10^{7}$ and $10^{12} \Omega \mathrm{cm}$. Large area RPCs with $2 \mathrm{~mm}$ single gaps operated in avalanche mode provide above $98 \%$ efficiency and a time resolution of around $1 \mathrm{~ns}$ up to a flux of several $\mathrm{kHz} / \mathrm{cm}^{2}$. These Trigger RPCs will, as an example, equip the muon detector system of the ATLAS experiment at CERN on an area of $3650 \mathrm{~m}^{2}$ and with 355.000 independent read out channels. Timing RPCs with a gas gap of 0.2 to $0.3 \mathrm{~mm}$ are widely used in multi gap configurations and provide $99 \%$ efficiency and time resolution down to $50 \mathrm{ps}$. While their performance is comparable to existing scintillator-based Time-Of-Flight (TOF) technology, Timing RPCs feature a significantly, up to an order of magnitude, lower price per channel. They will for example equip the $176 \mathrm{~m}^{2} \mathrm{TOF}$ barrel of the ALICE experiment at CERN with 160.000 independent read out cells.

RPCs were originally operated in streamer mode providing large signals which simplifies readout electronics and gap uniformity requirements. However, high rate applications and detector aging issues made the operation in avalanche mode popular. This was also facilitated by the development of new highly quenching $\mathrm{C}_{2} \mathrm{~F}_{4} \mathrm{H}_{2}$-based gas mixtures with small contents of $\mathrm{SF}_{6}$. While the physics of streamers is difficult to study, the avalanche mode opened the possibility for a detailed simulation of the detector physics processes in RPCs.

Even though RPCs were introduced in the early eighties and have been (will be) used in experiments, there are still disagreements about the explanation of several aspects of the RPC performance. The high efficiency of single gap RPCs would require a large ionization density of the used gases, which according to some authors contradicts measurements. Even in the case of a large ionization density the gas gain has to be extremely large, in order to arrive at the observed RPC efficiency. This raises other questions: A very strong space charge effect is required to explain the observed small avalanche charges around $1 \mathrm{pC}$. Doubts have been raised whether an avalanche can progress under such extreme conditions without developing into a streamer. To overcome these difficulties, other processes, like the emission of an electron from the cathode, were suggested. Moreover, the shape of measured charge spectra of single gap RPCs differs largely from what is expected from the statistics of the primary ionization and the avalanche multiplication.

In this thesis we discuss the detector physics processes of RPCs, from the primary ionization and the avalanche statistics to the signal induction and the read out electronics. We present Monte-Carlo simulation procedures that implement the described processes. While the fundament of the described model and some results were already published elsewhere [1] , the subject of this thesis is the implementation of the space charge effect. We present analytic formulas for the electrostatic potential of a point charge in the gas gap of an RPC. These formulas were developed in collaboration with
\end{abstract}


the University of Graz [2] and were published in [3, 4]. The simulation model presented in [1] is completed by the dynamic calculation of the space charge field using these formulas. Since the gas parameters like drift velocity and the Townsend and attachment coefficients depend on the electric field, they are calculated dynamically as well. The functional dependence of these parameters on the field is obtained with the simulation programs MAGBOLTZ and IMONTE. For the primary ionization parameters, we use the values that are predicted by the program HEED. While the described procedure only simulates the longitudinal avalanche development towards the anode of the RPC, we also present more dimensional models that allow a careful study of the transverse repulsive and attractive forces of the space charge fields, and of the consequences for the avalanche propagation.

We shall show that the efficiencies of single gap Timing RPCs is indeed explained by the high primary ionization density (about $9.5 / \mathrm{cm}$ as predicted by HEED) and a large effective Townsend coefficient (around $113 / \mathrm{mm}$ as predicted by IMONTE). We show that the space charge field reaches the same magnitude as the applied electric field in avalanches at large gas gain. This strong space charge effect effectively suppresses large values for the avalanche charges. The shape of the simulated charge spectra is very similar to the measurements. Also the simulated average charges are close to the experimental results. RPCs are operated in a strong space charge regime over a large range of applied voltage, contrary to wire chambers.

We apply only standard detector physics simulations to RPCs. The performance of Timing and Trigger RPCs is well reproduced by our simulations. The results concerning the space charge effect were presented and discussed at the 'RPC 2001' workshop [5] and on the '2002 NSS/MIC' conference [6]. 


\section{Contents}

1 Introduction 1

1.1 Particle Physics and Experiments . . . . . . . . . . . . . . 3

1.2 Interactions of Particles with Matter . . . . . . . . . . . . 8

1.2.1 Energy Loss due to Ionization and Excitation . . . . . . . . 9

$\llbracket 2.2$ Other Interaction Mechanisms of Radiation with Matter . . . 14

1.2 .3 Energy Loss and Particle Detection with RPCs . . . . . . . 18

1.3 Large Area Particle Detectors . . . . . . . . . . . . . . . . . . . . . 19

T.3.1 Time Resolution . . . . . . . . . . . . . . . . 20

1.3 .2 Spark Counter . . . . . . . . . . . . . . . 20

1.3.3 Paralle Plate Avalanche Chambers . . . . . . . . . 21

5.3 .4 Resistive Plate Chambers . . . . . . . . . . . . . . . . . . 22

1.4 Trigger RPCs and their Applications . . . . . . . . . . . . . . . . 25

1.5 Timing RPCs and their Applications $\ldots \ldots \ldots \ldots$

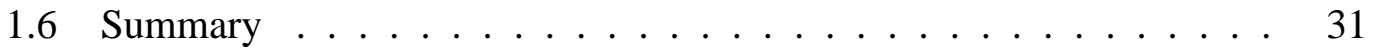

2 Detector Physics of RPCs 33

2.1 Gas Ionization by Fast Charged Particles . . . . . . . . . . . . . . . 34

2.1 .1 Distance between Primary Clusters $\ldots \ldots \ldots \ldots$

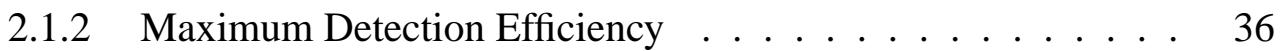

2.1.3 Cluster Size Distribution . . . . . . . . . . . . . . . . 37

2.2 Electron Drift and Multiplication . . . . . . . . . . . . . . . . . 37

2.2 .1 Thermal Motion and Diffusion . . . . . . . . . . . . . 38

2.2 .2 Electron Motion due to an Electric Field . . . . . . . . . . . 39

2.2 .3 Electron Multiplication . . . . . . . . . . . . . . . . . 40

2.2 .4 Avalanche Statistics . . . . . . . . . . . . . . . . 41

2.3 Electrostatics of Three Layer Geometries _. . . . . . . . . . . 47

2.3.1 Potential of a Point Charge for the Three Layer Problem . . . 47

2.3.2 Electric Field of a Point Charge for the Three Layer Problem . 51

2.4 Signal Induction Process $\ldots \ldots \ldots \ldots \ldots \ldots$

2.4 .1 Weighting Field in the Gas Gap of an RPQ . . . . . . . . 55

2.4 .2 Induced Charge . . . . . . . . . . . . . . . . . . . 58

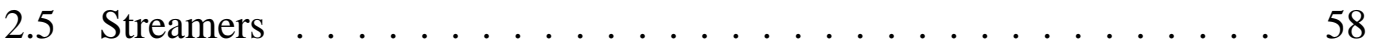

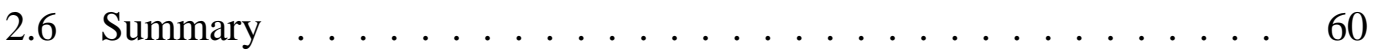


3 Monte Carlo Avalanche Simulation 63

3.1 The I-D Model . . . . . . . . . . . . . . . . . . . . . 64

3.2 The 1.5 -D Model . . . . . . . . . . . . . . . 66

3.2.1 Longitudinal Diffusion . . . . . . . . . . . . . . 67

$\$ .2 .2$ Transverse Diffusion $\ldots \ldots \ldots \ldots \ldots \ldots$

3.2 .3 Space Charge Effect . . . . . . . . . . . . . . . . . . . . . . 69

3.2.4 Electrons in the Anode Resistive Layer . . . . . . . . . . 72

3.2.5 Field Dependence of the Electron Multiplication . . . . . . 72

3.2 .6 Field Dependence of the Drift Velocity . . . . . . . . . . . 74

3.2.7 Induced Current Signal and Induced Charge . . . . . . . . . 76

3.3 The 2-D Modell . . . . . . . . . . . . . . . . . . 77

3.3.1 Calculation of the Electric Field Vector . . . . . . . . . 78

3.3 .2 Propagation of the Charges . . . . . . . . . . . . 80

3.4 The 3 -D Model . . . . . . . . . . . . . . . 82

3.4.1 Convergence of the 3-D Model . . . . . . . . . . . . 83

B.4.2 Drawbacks of the 3-D Model . . . . . . . . . . . . . 86

3.5 Summary $\ldots \ldots \ldots \ldots \ldots \ldots \ldots \ldots$

\begin{tabular}{|lll}
4 & Geometries and Typical Operating Parameters & 87
\end{tabular}

5 Results Obtained with the 1-D Modell 93

$5.1 \quad$ Efficiency and Time Resolution . . . . . . . . . . . . . . . . . 93

5.2 Charge-to-Time Correlation . . . . . . . . . . . . . . . . . . . 97

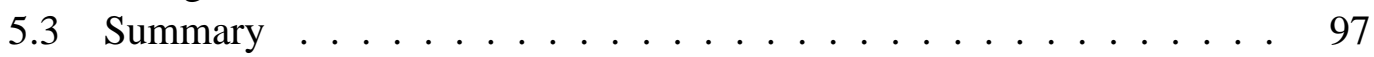

6 Results Obtained with the 1.5-D Model 99

$6.1 \quad$ Signal Rise Time $\ldots \ldots \ldots \ldots \ldots$

6.2 Charge Spectra . . . . . . . . . . . . . . . . . . . . 102

6.2 .1 Timing RPCs with $0.3 \mathrm{~mm}$ Gaps $\ldots \ldots \ldots \ldots$

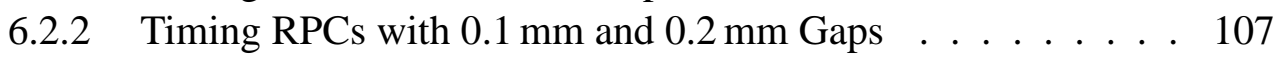

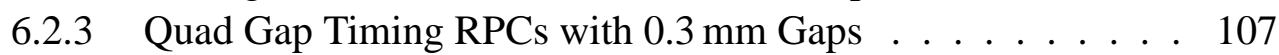

6.2 .4 Trigger RPCs with $2 \mathrm{~mm} \mathrm{Gaps} \ldots \ldots \ldots \ldots \ldots$

6.3 Operational Mode of RPCS . . . . . . . . . . . . . . . . . 113

6.4 Charge-to-Time Correlation . . . . . . . . . . . . . . . . . . . 115

6.5 Avalanches in Pure lsobutane . . . . . . . . . . . . . . . . 117

6.6 Streamers . . . . . . . . . . . . . . . . . . . . . 120

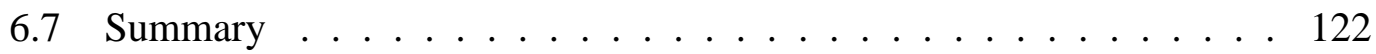

7 Results Obtained with the 2-D Model 123

7.1 Comparison of the Different Moders . . . . . . . . . . . . . 123

7.2 Avalanches in $\mathrm{C}_{2} \mathrm{~F}_{4} \mathrm{H}_{2} / \mathrm{i}_{2} \mathrm{C}_{4} \mathrm{H}_{10} / \mathrm{SF}_{6} \ldots \ldots \ldots \ldots \ldots$

7.2 .1 Electron Density . . . . . . . . . . . . . . . . . . . . . 126

7.2 .2 Total Ion Density . . . . . . . . . . . . . . . . . . . . . . 128 
7.2 .3 Total Charge Density . . . . . . . . . . . . . . . . . . . 130

7.2 .4 Electric Field . . . . . . . . . . . . . . . . . . . . . . 132

7.2 .5 Drift Velocity . . . . . . . . . . . . . . . . . . . . . 135

T.2.6 Effective Townsend Coefficient . . . . . . . . . . . 137

7.2 .7 Longitudinal Electric Field . . . . . . . . . . . . . . . . . . 139

7.2.8 RadialElectric Field . . . . . . . . . . . . . . . . . 141

7.2 .9 Radial Drift Velocity . . . . . . . . . . . . . . . . . . . . . 145

7.3 Avalanches in Pure lsobutane . . . . . . . . . . . . . . . . . 147

7.3 .1 Early Stage of the Avalanches . . . . . . . . . . . . . . 147

7.3 .2 Later Stages of the Avalanches . . . . . . . . . . . . . . . 149

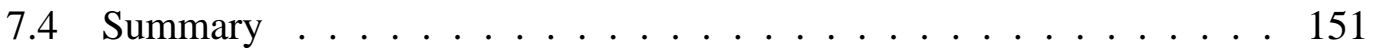

8 Conclusions and Outlook 155

\begin{tabular}{lll}
\hline D DifferentialCollision Cross Sections & 157
\end{tabular}

A.1 Electron-Electron Scattering . . . . . . . . . . . . . . . . . . 157

A.2 Positron-Electron Scattering $\ldots \ldots \ldots \ldots$

A.3 Scattering of Massive Spin 0 Particles off Electrons . . . . . . . . . 158

4 A.4 Scattering of Massive Spin $\frac{1}{2}$ Particles off Electrons . . . . . . . . . . 159

A.5 Scattering of Massive Spin 1 Particles off Electrons . . . . . . . . . 159

A.6 Collision Cross Section Dependence on the Spin . . . . . . . . . . 159

\begin{tabular}{lll}
\hline Überblick & 161
\end{tabular}

B. Motivation für die Arbeit . . . . . . . . . . . . . . . . . 163

B.2 Detektorphysik von RPCs . . . . . . . . . . . . . . . . . . 163

B.3 Ergebnisse . . . . . . . . . . . . . . . . . . . . 166

B.4 Schlussfolgerung und Ausblick . . . . . . . . . . . . . . . 167 


\section{List of Figures}

1.1 Schematic image of an avalanche . . . . . . . . . . . . . 2

1.2 Components of a typical high energy physics experiment . . . . . . 7

1.3 Energy loss of muons in copper . . . . . . . . . . . . . . . . 13

1.4 Energy loss due to ionization and excitation in different materials . . . 19

1.5 Schematic image of an RPC geometry . . . . . . . . . . . . 23

1.6 Schematic image of the development of an avalanche in an RPQ . . . 23

1.7 Schematic image of the development of a streamer . . . . . . . . . 24

1.8 View of the Large Hadron Collider . . . . . . . . . . . . . . . 26

L.9 View of the ACLAS detector . . . . . . . . . . . . . . . 27

1.10 Transverse view of the ATLAS muon spectrometer . . . . . . . . 28

1.11 Crossection of a single gap Trigger RPC . . . . . . . . . . . . . . . . 28

1.12 The ALICE detector and the TOF system . . . . . . . . . . . . . . . 29

1.13 Particle separation with a Time-of-Flight detector . . . . . . . . . 30

1.14 The ALICE multigap Timing RPCS . . . . . . . . . . . . . . 30

2.1 Coordinate system used for the RPC studies . . . . . . . . . . . . . . . . 34

2.2 Average number of cluster per mm for typical gases . . . . . . . . . 36

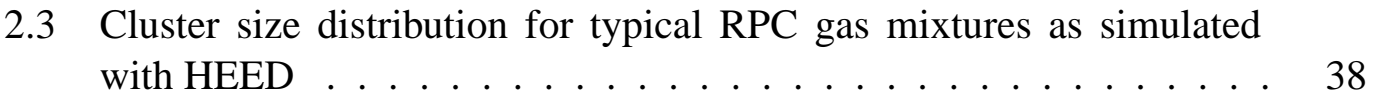

2.4 Drift velocity as calculated with MAGBOLTZ . . . . . . . . . . . . . 39

2.5 Diffusion coefficients calculated with MAGBOLTZ . . . . . . . . . 41

2.6 Cloud chamber photograph of electron avalanches . . . . . . . . . . 42

2.7 Charge distributions for avalanches starting with a single electron. . . 43

2.8 Townsend and attachment coefficients for different gas mixtures . . . 46

2.9 RPC geometry . . . . . . . . . . . . . . . . . . . . . . 47

2.10 Potential of a point charge in an RPC . . . . . . . . . . . . . . . . . 49

2.11 Convergence of the integrand term . . . . . . . . . . . . . 49

2.12 The different terms for the potential of a point charge in an RPC . . . 50

2.13 Dependence of the space charge field on the relative permittivity . . . 50

$2.14 z$-component of the electric field of a point charge in an RPC . . . . . 52

2.15 Comparison of the full electric field solution to the field of free point

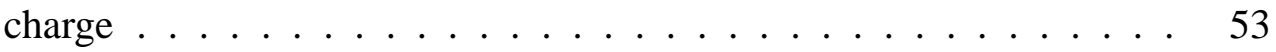

2.16 Comparison of the electric field solution to the field of free point charge and one mirror charge . . . . . . . . . . . . . 53 
2.17 The weighting field mechanism to calculate induced currents . . . . 55

2.18 RPC geometry with strips . . . . . . . . . . . . . . . . 56

$2.19 z$-and $x$-component of the weighting field $\ldots \ldots \ldots . \ldots 57$

2.20 Precursor and streamer signal . . . . . . . . . . . . . . . . . 59

3.1 Avalanches started by one electron at the same position . . . . . . . 65

3.2 Examples for avalanches with longitudinal diffusion . . . . . . . . . 68

3.3 Geometry for the 1.5-D simulation . . . . . . . . . . . . . . 70

3.4 Comparison of the potentials of a point charge and transverse charge distributions . . . . . . . . . . . . . . 70

3.5 The influence of the step size on the simulation . . . . . . . . . 71

3.6 Comparison of the fields of two transverse Gaussian charge distribu-

tions with different standard deviations . . . . . . . . . . . . . . . . 72

3.7 Avalanches with space charge effect . . . . . . . . . . . . . . 73

3.8 Snapshots of a simulated avalanches with space charge effect . . . . . 75

3.9 Example avalanches with longitudinal repulsive/attractive forces . . . 76

3.10 Geometry for calculating the electric field of a charge ring . . . . . 78

3.11 Plots of the elliptic integrals of the first kind and of the second kind $\quad$. 80

3.12 The coordinate system and the electric field vector in the two dimen-

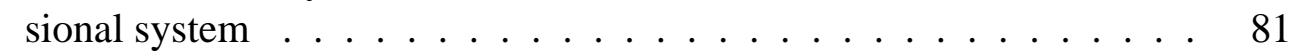

3.13 Electron density in the 3-D simulation . . . . . . . . . . . 85

4.1 Schematic images of Timing RPCs . . . . . . . . . . . . . . . . 88

4.2 Calculated Timing RPC spectra . . . . . . . . . . . . . . . 92

$5.1 \quad$ Simulated efficiencies and time resolution of Timing RPCs . . . . . . 94

5.2 Simulated efficiencies and time resolution of Trigger RPCs . . . . . . 95

5.3 Measured charge-to-time correlation . . . . . . . . . . . . 96

5.4 Correlation of the charge threshold crossing time to the induced charge with read out electronics, simulation . . . . . . . . . . . . . . . . 96

6.1 Influence of the space charge effect on the Timing RPC time resolution 100

6.2 Influence of the space charge effect on the Trigger RPC time resolution 100

6.3 Some simulated avalanches in Timing RPCs . . . . . . . . . . . . 101

$6.4 \quad$ Simulated and measured value of $(\alpha-\eta) v_{D} \ldots \ldots$. . . . . . . . . 102

6.5 Simulated Timing RPC charge spectrum without space charge effect . 103

6.6 Simulated avalanche in a Timing RPG . . . . . . . . . . . . . . 105

6.7 Simulated signal charge spectra for Timing RPCs, $0.3 \mathrm{~mm}$ gap . . . . 106

6.8 Comparison of simulated and measured spectra of the signal charge for Timing RPCs, $0.3 \mathrm{~mm}$ gap $\ldots \ldots \ldots \ldots 6$

6.9 Comparison of simulated and measured spectra of the signal charge

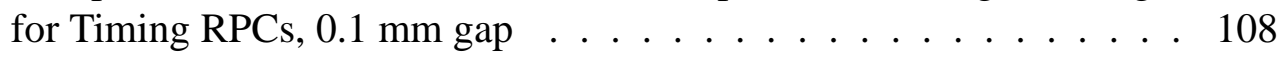

6.10 Simulated spectra of the total signal charge for Timing RPCs, $0.2 \mathrm{~mm}$ gap . . . . . . . . . . . . . . . . 108 
6.11 Simulated induced charge spectra for a quad gap Timing RPC . . . . 109

6.12 Simulated avalanche in a Trigger RPC . . . . . . . . . . . . . . 111

6.13 Simulated induced charge spectra for Trigger RPCs . . . . . . . . . . 112

6.14 Simulated total signal charge spectra for Trigger RPCs . . . . . . . . 112

6.15 Average total signal charges of Timing RPCs versus the applied elec-

tric tield . . . . . . . . . . . . . . . . . . 113

6.16 Average induced charges of Timing RPCs versus the applied electric

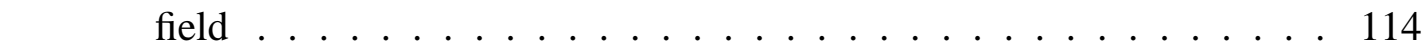

6.17 Intrinsic correlation of the charge threshold crossing time to the in-

6.18 Correlation of the total signal charge to the position of the formation of the first cluster that reached the anode . . . . . . . . . . . . . . . 116

6.19 Charge-time correlations for avalanches started by one electron . . . . 117

6.20 Simulated avalanche in a Timing RPC filled with pure isobutane . . . 118

6.21 Comparison of simulated and measured spectra of the signal charge

for Timing RPCs with pure isobutane, $0.3 \mathrm{~mm}$ gap $\ldots . . . . .119$

6.22 Total number of charge carriers in simulated avalanches and maximum value of the electric field . . . . . . . . . . . . . . . . 121

7.1 Comparison of avalanches simulated with the different models . . . . 124

7.2 Electron distribution . . . . . . . . . . . . . . . . . . . 127

7.3 Electron distribution, contour plot $\ldots \ldots \ldots$

7.4 lon distribution . . . . . . . . . . . . . . . . . . . . . . . . . . . . 129

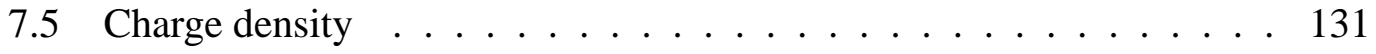

7.6 Absolute value of the space charge field . . . . . . . . . . . 133

7.7 Absolute value of the soace charge field sensed by the electrons . . . 134

7.8 Drift velocity in the gas gap . . . . . . . . . . . . . . 136

7.9 Effective Townsend coefficient in the gas gap $\ldots \ldots \ldots$

$7.10 z$-component of the space charge field . . . . . . . . . . . . 140

$7.11 z$-component of the space charge field sensed by the electrons . . . . 142

$7.12 r$-component of the space charge field $\ldots \ldots \ldots \ldots . \ldots 144$

$7.13 r$-component of the space charge field sensed by the electrons . . . . . 145

$7.14 r$-component of the drift velocity sensed by the electrons . . . . . . . 146

7.15 Several parameters in an avalanche in pure isobutane, early stage . . . 148

7.16 Several parameters in an avalanche in pure isobutane, later stage . . . 150

7.17 Several parameters in an avalanche in pure isobutane, final stage . . . 152 


\section{Chapter 1}

\section{Introduction}

Resistive Plate Chambers (RPCs) are gaseous parallel plate avalanche detectors with time resolutions down to $50 \mathrm{ps}$, making them attractive for trigger and Time-Of-Flight applications, in some cases covering large areas up to a few thousand square meters. Advantages compared to other technologies are the robustness and simplicity of construction. They are also well adapted to inexpensive industrial production. RPCs were originally operated in streamer mode providing large signals, which simplifies read out electronics and gap uniformity requirements. However, high rate applications and detector aging issues made the operation in avalanche mode popular. This was also facilitated by the development of new highly quenching $\mathrm{C}_{2} \mathrm{~F}_{4} \mathrm{H}_{2}$-based gas mixtures. While the physics of streamers is difficult to study, the avalanche mode opened the possibility for detailed simulations of the detector physics processes in RPCs.

The fundamental processes that underly the operation of RPCs are well known: a charged particle leaves free charge carriers in the gas, which are drifted towards the anode and multiplied by an appropriate electric field. The propagation of the growing number of charges induces a signal on a read out electrode. The high voltage that is applied to the parallel plate electrodes leads to a uniform electric field in the gas gap. However, there are still disagreements about the explanation of several aspects of the performance of RPCs [7]. Especially since the introduction of Timing RPCs with gas gaps of a few hundred microns and very high applied field strengths $(\approx 100 \mathrm{kV} / \mathrm{cm})$ in the last years of the 20th century, a seeming disagreement between the high efficiencies of the device and the rather low observed signal charges was observed. To explain the observed detection efficiencies, a large density of primary clusters in the used gases is necessary, which according to some authors contradicts experimental values [8, 9]. A large ionization density leads to a higher probability for the deposit of electrons close to the cathode from where avalanches cross almost the whole gas gap and can thus reach sufficient sizes to cross the threshold. Even in the case of a large ionization density the gas gain has to be extremely large to increase the number of avalanches that cross the threshold and thus explain the observed efficiencies. This raises other questions: Assuming exponential growth of the avalanches, the average 


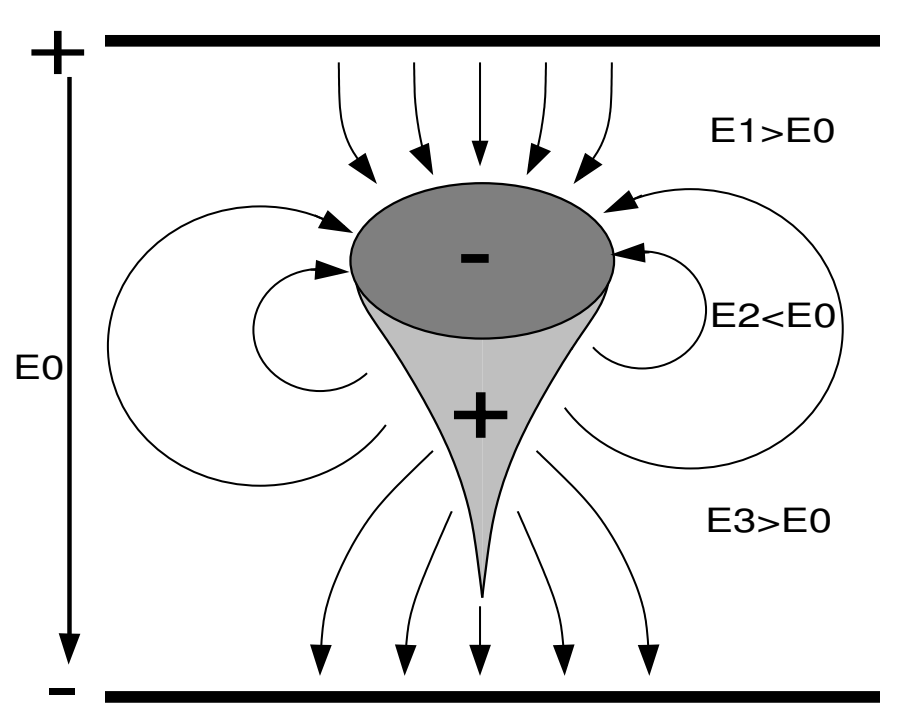

Figure 1.1: A schematic picture of an avalanche and the electric field deformations caused by the avalanche charge carriers. At the tip and the tail of the charge distribution the fields $E_{1}$ and $E_{3}$ are higher than the applied electric field $E_{0}$. In the center of the charge distribution the field $E_{2}$ is lower than $E_{0}$. As a consequence, the value of the gas parameters like drift velocity and Townsend coefficient may vary with the position in the gas gap.

avalanche charge would be up to seven orders of magnitude larger than the measured values. A very 'strong' space charge effect is required to explain the small observed charges of around $1 \mathrm{pC}$ [10] and doubts have been raised whether an avalanche can progress under such extreme conditions without developing into a streamer [9]. The space charge effect is shown schematically in Fig. 1.1. Some authors propose that other mechanisms like the extraction of surface electrons by the incident particle from the detector frame contribute to the detection efficiency of the device [8].

Another disagreement concerns the shape of the charge spectra. While the statistics of avalanche multiplication predicts a shape following a power law, measurements show a peak that is becoming more pronounced with higher voltages. Many authors applied the so-called Polya distribution to the statistics of avalanche fluctuations in RPCs. This model assumes that the probability for the multiplication of electrons depends on the current size of the avalanche, which includes some kind of space charge effect in an incorrect way.

In this thesis we discuss in detail the detector physics processes that are relevant for the operation of RPCs. We present Monte-Carlo simulation procedures that implement the described processes, from the primary ionization and the avalanche statistics to the induced signals and the front end electronics. A simple one dimensional simulation model without diffusion and space charge effects and results obtained with that model 
were published in [1]]. The subject of this thesis is the implementation of the space charge effect into this model.

In this introduction we give a short overview on modern high energy physics experiments and the different tasks the detector subsystems have to perform (section 1.1). Then we will summarize very briefly the fundamentals of the interactions of particles with matter (section 1.2) and the history of the development of particle detectors in general and the Resistive Plate Chamber in particular (section 1.3). Finally we present the two different types of RPCs that are commonly used today: the Trigger RPC (section (1.4) and the Timing RPC (section 1.5), and how they are implemented in present and future high energy physics experiments.

In chapter 2 the fundamental detector physics of RPCs are described in detail and in chapter 3 we describe four different simulation models based on those physical effects. In chapters 5, 6 and 7 we finally present the results that were obtained with the different models and for different detector geometries.

\subsection{Particle Physics and Experiments}

Modern high energy physics experiments with accelerators generally use a beam of charged particles. This beam is either focused on a fixed target or collides with another beam of opposite direction. The experiments built around the interaction point(s) are complex systems made of many layers of different particle detectors, each with a specific task. Each of the detectors produces electrical signals that contain information about the path of a particle, the energy it deposited, or the time at which it passed through. All the gathered information must be pieced together, ultimately to reveal the particles that were created by the high energy collision and that might have lifetimes too short to ever show a visible track.

The first fundamental particle to be discovered was the electron. In 1897 J.J. Thomson performed a series of experiments to prove conclusively that the mysterious cathode rays, discovered some years before, are indeed streams of negatively charged particles with a mass approximately two thousand times less than the mass of a hydrogen atom. Another building brick of matter, the proton, was discovered around 1911 by E. Rutherford and E. Marsden when they scattered alpha particles from atomic nuclei. Rutherford concluded that the protons, first known as 'H particles', were the carriers of the positive charge in the nucleus. If they were the only constituents, a nucleus with twice the charge of another should also have twice the mass. This is not so. Nuclei have at least double the mass expected from the number of protons suggested by the total charge. Rutherford speculated in 1920 that there are electrically neutral particles within nuclei: the neutrons. While at that time most physicists accepted the idea that there were protons and electrons in the nucleus, the neutron was finally discovered in 1932 by J. Chadwick when he bomarded beryllium with alpha particles from a polo- 


\begin{tabular}{|c|c|c|c|c|c|}
\hline particle & & constituents & Mass [MeV] & Lifetime $\tau$ [s] & $c \tau$ \\
\hline "Electron/Positron & $\mathrm{e}^{ \pm}$ & & 0.511 & $\infty$ & $\infty$ \\
\hline (Anti)Muon & $\mu^{ \pm}$ & & 105.7 & $2.2 \times 10^{-6}$ & $659 \mathrm{~m}$ \\
\hline (Anti)Tauon & $\tau^{ \pm}$ & & 1777 & $2.9 \times 10^{-13}$ & $87 \mu \mathrm{m}$ \\
\hline Electron-Neutrino & $\nu_{e}$ & & $<3 \times 10^{-6 *}$ & $\infty$ & $\infty$ \\
\hline Muon-Neutrino & $\nu_{\mu}$ & & $<0.19^{*}$ & $\infty$ & $\infty$ \\
\hline Tau-Neutrino & $\nu_{\tau}$ & & $<18.2^{*}$ & $\infty$ & $\infty$ \\
\hline Photon & $\gamma$ & & 0 & $\infty$ & $\infty$ \\
\hline Charged Pions & $\pi^{ \pm}$ & $u \bar{d}, d \bar{u}$ & 140 & $2.6 \times 10^{-8}$ & $7.8 \mathrm{~m}$ \\
\hline Charged kaons & $\mathrm{K}^{ \pm}$ & $\overline{u s}, s \bar{u}$ & 494 & $1.2 \times 10^{-8}$ & $3.7 \mathrm{~m}$ \\
\hline Neutral kaons & $\begin{array}{l}\mathbf{K}_{L}^{0}, \\
\mathbf{K}_{S}^{0}\end{array}$ & $d \bar{s}, s \bar{d}$ & 497 & $\begin{array}{l}5.1 \times 10^{-8}, \\
8.9 \times 10^{-11}\end{array}$ & $\begin{array}{l}15.5 \mathrm{~m}, \\
2.7 \mathrm{~cm}\end{array}$ \\
\hline D-Mesons & $\begin{array}{l}\mathrm{D}^{ \pm} \\
\mathrm{D}^{0} \\
\mathrm{D}_{s}^{ \pm}\end{array}$ & $\begin{array}{l}c \bar{d}, d \bar{c} \\
c \bar{u}, u \bar{c} \\
c \bar{s}, s \bar{c}\end{array}$ & $\begin{array}{l}1869 \\
1864 \\
1969\end{array}$ & $\begin{array}{l}1.0 \times 10^{-12} \\
4.1 \times 10^{-13} \\
4.9 \times 10^{-13}\end{array}$ & $\begin{array}{l}315 \mu \mathrm{m} \\
123 \mu \mathrm{m} \\
147 \mu \mathrm{m}\end{array}$ \\
\hline B-Mesons & $\begin{array}{l}\mathrm{B}^{ \pm} \\
\mathrm{B}^{0} \\
\mathrm{~B}_{s}^{0} \\
\mathrm{~B}_{c}^{ \pm}\end{array}$ & $\begin{array}{l}u \bar{b}, b \bar{u} \\
b \bar{d}, d \bar{b} \\
s \bar{b}, b \bar{s} \\
c \bar{b}, b \bar{c}\end{array}$ & $\begin{array}{c}5279 \\
5279 \\
5370 \\
\sim 6400\end{array}$ & $\begin{array}{c}1.7 \times 10^{-12} \\
1.5 \times 10^{-12} \\
1.5 \times 10^{-12} \\
\sim 5.0 \times 10^{-13}\end{array}$ & $\begin{array}{c}502 \mu \mathrm{m} \\
462 \mu \mathrm{m} \\
438 \mu \mathrm{m} \\
\sim 150 \mu \mathrm{m}\end{array}$ \\
\hline Proton & $\mathrm{p}$ & $u u d$ & 938.3 & $>10^{25} \mathrm{y}$ & $\infty$ \\
\hline Neutron & $\mathrm{n}$ & $u d d$ & 939.6 & $885.7 \mathrm{~s}$ & $10^{8} \mathrm{~km}$ \\
\hline Lambda & $\begin{array}{c}\Lambda \\
\Lambda_{c}^{+} \\
\Lambda_{b}\end{array}$ & $\begin{array}{l}u d s \\
u d c \\
u d b\end{array}$ & $\begin{array}{l}1116 \\
2285 \\
5624\end{array}$ & $\begin{array}{l}2.6 \times 10^{-10} \\
2.0 \times 10^{-13} \\
1.2 \times 10^{-12}\end{array}$ & $\begin{array}{c}7.9 \mathrm{~cm} \\
60 \mu \mathrm{m} \\
368 \mu \mathrm{m}\end{array}$ \\
\hline Sigma & $\begin{array}{l}\Sigma^{+} \\
\Sigma^{-}\end{array}$ & $\begin{array}{l}\text { uus } \\
d d s\end{array}$ & $\begin{array}{l}1189 \\
1198\end{array}$ & $\begin{array}{l}8.0 \times 10^{-11} \\
1.5 \times 10^{-10}\end{array}$ & $\begin{array}{l}2.4 \mathrm{~cm} \\
4.4 \mathrm{~cm}\end{array}$ \\
\hline $\mathrm{Xi}$ & $\begin{array}{l}\Xi^{0} \\
\Xi^{-} \\
\Xi_{c}^{+} \\
\Xi_{c}^{0}\end{array}$ & $\begin{array}{l}\text { uss } \\
d s s \\
u s c \\
d s c\end{array}$ & $\begin{array}{l}1315 \\
1321 \\
2466 \\
2472\end{array}$ & $\begin{array}{c}2.9 \times 10^{-10} \\
1.6 \times 10^{-10} \\
4.4 \times 10^{-13} \\
\sim 1.0 \times 10^{-13}\end{array}$ & $\begin{array}{l}8.7 \mathrm{~cm} \\
4.9 \mathrm{~cm} \\
132 \mu \mathrm{m} \\
\sim 29 \mu \mathrm{m}\end{array}$ \\
\hline Omega & $\begin{array}{l}\Omega^{-} \\
\Omega_{c}^{0}\end{array}$ & $\begin{array}{l}s s s \\
s s c\end{array}$ & $\begin{array}{l}1673 \\
2698\end{array}$ & $\begin{array}{l}8.2 \times 10^{-11} \\
6.0 \times 10^{-14}\end{array}$ & $\begin{array}{l}2.5 \mathrm{~cm} \\
19 \mu \mathrm{m}\end{array}$ \\
\hline
\end{tabular}

Table 1.1: All known particles with a mean lifetime larger than $\sim 10^{-13} \mathrm{~s}$ [11]]. We find the three charged leptons, the neutrinos, the photon, ten mesons and 13 baryons. The corresponding antiparticles of the baryons and of the neutrinos are not listed. The lifetimes of particles and their antiparticles are equal. For the hadrons the quark constituents are shown: $u=$ up-quark, $d=$ down-quark, $c=$ charm-quark and $s=$ strangequark.

*) There is increasing evidence from neutrino-oscillation experiments that neutrinos have a finite rest mass. 
nium source. He allowed the neutral particles emerging from the beryllium to collide with a variety of gases. By observing the differing amounts by which the atomic nuclei in the different gases recoiled, he could calculate that the neutrons had more or less the same mass as the proton.

The carrier of the electromagnetic force, the photon, was discovered by A. Compton in 1923, when he performed scattering experiments with X-rays and a carbon block. His results could only be explained if one assumed collisions between lightparticles, the photons, with both definite energy and a definite momentum, and atomic electrons.

Two more particles were discovered in the cosmic rays in the 1930s: first evidence of a positron was obtained in a cloud chamber photograph in 1932 by C. Anderson; the muon was discovered in 1937 by C. Anderson and S. Neddermeyer, with a cloud chamber that was triggered by a Geiger counter. By 1952 cloud chambers exposed to cosmic rays had revealed yet more new particles: the first examples of 'V-particles' were observed in cloud chamber photographs triggered by Geiger counters between sheets of lead by G. Rochester and C. Butler in 1947. Those particles had about half the mass of the proton and later became known as the charged and neutral kaons. Another neutral V-particle with a mass larger than the proton is called the lambda. Together, the kaons and the lambda became known as the 'strange' particles because their behaviour was unexpected. Another strange particle, the negative xi or cascade particle, was discovered shortly after the lambda (R. Armenteros et al., 1952).

In the late 1940s, the development of special photographic emulsions, which could easily be carried aloft by baloons, brought the first images of high altitude cosmic rays. This led to the discovery of the charged Pions by C. Powell (1947). Yet another strange particle, the sigma plus, was discovered using the emusion technique (G. Tomasini et al., 1953).

The year 1952 was the beginning of a new era in particle physics. It saw the invention of a new type of detector: the bubble chamber; and it witnessed the first of a new breed of accelerator: the synchrotron. The first particle to be discoverd at an accelerator, the neutral pion, completed the pion family of three (R. Bjorkland et al., 1949). From now on many more particles were discovered at accelerators, starting with the sigma minus (W. Fowler et al., 1953), the antiproton (W. Segrè et al., 1955) and the antineutron (B. Cork et al., 1956).

The invention of the multiwire proportional chamber (G. Charpak, 1968 [12]) and the availability of transistors made the construction of fast and precise electronic particle detectors possible. Electronic counter experiments led to the discovery of hundreds of more particles. Table 1.1 shows all known particles with a mean lifetime sufficiently large to travel more than about $10 \mu \mathrm{m}$ (at GeV energies) before they decay [11]. If the mean lifetime is smaller than that, the particle can not be directly seen in a detector.

In Table 1.1 we find 30 particles. The three neutrinos interact only weakly and are in general not seen in a detector. Twelve of the remaining 27 particles stray no 
more than $0.5 \mathrm{~mm}$ from the collision point before they decay. In the case of a colliding beam experiment they do not even have time to escape from the beam pipe. They can only be detected by extrapolating the very precisely measured tracks of the more stable decay products to the secondary vertex (displaced vertex), where they decayed, close to the collision point'. From the remaining 15 particles the following eight (plus the corresponding antiparticles) are by far the most frequent ones:

electrons, muons, photons, charged pions, charged kaons, neutral kaons, protons and neutrons.

This leads us to a very basic insight:

The task of modern high energy physics detector systems is to identify eight different particles (and the corresponding antiparticles) that are crossing the device and to measure their momenta and/or energy. The same task is repeatedly implemented in similar ways in all high energy physics experiments.

Fig. 1.2 shows the basic setup of many modern high energy physics experiments. The reason that detectors are divided into many components is that each component tests for a special set of particle properties. These components are stacked such that all particles will go through the different layers sequentially. We summarize the different tasks of the detector subsystems:

Tracking Chambers: Directions, momenta, and signs of charged particles have to be measured. Finely subdivided tracking detectors are used to reconstruct charged particle trajectories. A magnetic field causes the trajectories to bend in circular paths: the radius of each circle determines the momentum, and the 'bending direction' the sign of charge.

Electromagnetic Calorimeter: The energy carried by electrons and photons is measured by the electromagnetic calorimeter. It is generally subdivided into segments that absorb the energy of incident electrons and photons, and produce signals proportional to that energy.

Hadronic Calorimeter: The energy carried by hadrons (protons, pions, neutrons, etc.) is measured by the hadronic calorimeter. It detects hadronic showers in a similar way as the electromagnetic calorimeter detects electromagnetic showers. The hadronic calorimeter is always downstream (outside) of the electromagnetic calorimeter, due to the much larger interaction length of hadrons.

\footnotetext{
${ }^{1}$ The identification of such a displaced vertex can be used for the tagging of events ( $\tau-$, D- or Btagging) or even for triggering like in the LHCb experiment at CERN [13, 14].
} 


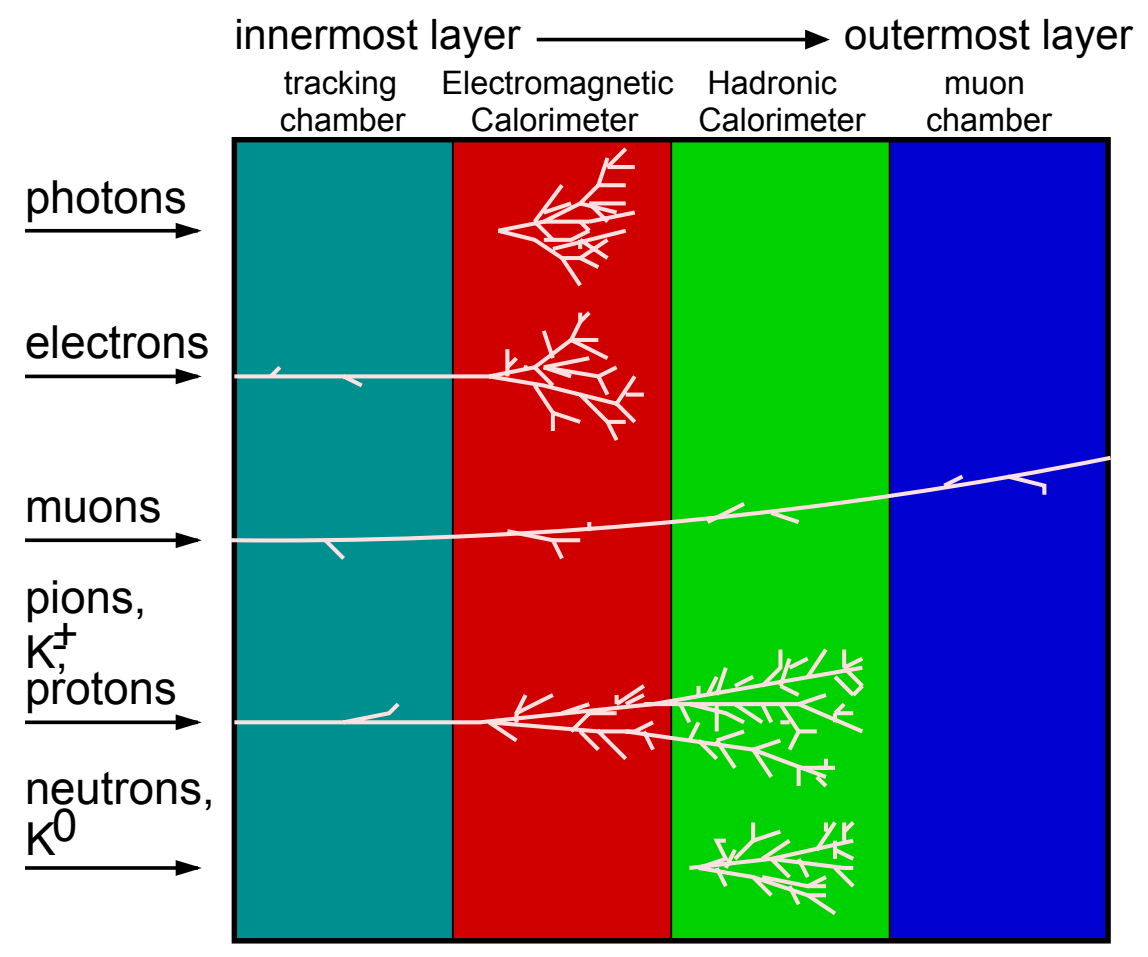

Figure 1.2: The different main components of a typical detector. The charged particles (electrons, muons, protons, charged kaons $\left(\mathrm{K}^{ \pm}\right)$and charged pions) are detected both in the tracking chamber and the electromagnetic calorimeter. The neutral particles (neutrons, photons and neutral kaons $\left(\mathrm{K}^{0}\right)$ ), leave no trail in the tracking chamber. Photons are detected by the electromagnetic calorimeter, while neutrons and $\mathrm{K}^{0} \mathrm{~s}$ are evidenced by the energy they deposit in the hadron calorimeter. Each particle type has its own signature in the detector. For example, if a particle is detected only in the electromagnetic calorimeter, it is fairly certain that it is a photon.

Muon System: High energy muons are the only charged particles that penetrate large amounts of matter. In doing so they suffer only small deflections from their original direction of motion, and lose little of their energy. Muons are generally detected in tracking detectors downstream (outside) of the calorimeters.

Often more detector systems are added to provide more information on the different particles:

Particle Identification (PID): The system generally has to provide information for the identification of the different charged and neutral particles. Charged particles can be identified by combining the momentum information from the tracking detectors and the independently measured velocity (Time-Of-Flight, TOF), energy loss $d E / d x$, Črenkov radiation or transition radiation. 
Displaced Vertex: Often it is important to identify charged particles that originate at points a short distance from the collision point rather than at the collision point itself (B-, D- or $\tau$-tagging). This is achieved with high spatial resolution detectors placed around the collision point.

Neutrinos: The presence of the not directly detectable neutrinos can be infered through momentum conservation.

A particle will not be evident until it either interacts with the detector in a measurable fashion, or decays into detectable particles. Despite their differences the detector types that were just described all rely on the same basic principles. Particle detectors make visible the effects that the particles have on their surroundings. In the next section we will give a brief summary of the different ways in which particles interact with matter.

\subsection{Interactions of Particles with Matter}

In the last section we mentioned that a particle detector has to be able to reveal the presence of eight particles (and their corresponding antiparticles): electrons, muons, protons, neutrons, photons, charged pions, charged kaons and neutral kaons. These particles leave characteristic trails as they lose energy when they travel through a material, be it a gas, a liquid or a solid. This energy loss can be of different forms:

- Electrically charged particles lose energy by 'colliding' with atomic electrons of the material (excitation, ionization) and by the emission of bremsstrahlung when they scatter off the nuclei.

- Strongly interacting particles can in addition lose energy through hadronic interactions (inelastic nuclear collisions, nuclear excitation, splitting).

- Photons lose energy by Compton scattering with atomic electrons or they disappear completely in the processes of Photo Electric Effect and pair production.

In this section the basic interaction mechanisms of particles with matter are summarized briefly. The energy loss of charged particles due to ionization and excitation is fundamental to most particle detectors - and the RPC, that is the topic of this thesis - and is therefore described in more detail. 


\subsubsection{Energy Loss due to Ionization and Excitation}

We consider a relativistic charged particle scattering on atomic electrons, e.g.

$$
\mu^{+}+\text {atom } \rightarrow \mu^{+}+\text {atom }^{+}+\mathrm{e}^{-} .
$$

If the distance of closest approach is large compared to the size of the atom (a distant collision), the atom will react 'as a whole' to the variable electromagnetic field of the charged particle. The result can be excitation or ionization of the atom. If the distance of closest approach is of the order of the atomic dimensions, (a close collision) the interaction involves the passing particle and one of the atomic electrons. As a consequence, the electron is ejected from the atom with considerable energy (knock-on electrons). We define [15]

distant collisions: Any collision resulting in the ejection of an electron of energy smaller than a predetermined value $\nu$.

close collisions: Any collision resulting in the ejection of an electron of energy larger than $\nu$. If $\nu$ is sufficiently large (and the corresponding impact parameter sufficiently small) we can treat all close collisions by considering the atomic electrons as free particles.

A limiting energy $\nu$ of 10 to $100 \mathrm{keV}$ simultaneously satisfies the two conditions specified above for practically all cases of importance in the field of high energy phenomena.

\section{The Differential Collision Cross Section}

We note the atomic differential cross section that a particle with energy $E$ loses an energy between $E^{\prime}$ and $E^{\prime}+d E^{\prime}$ in a collision with an atom

$$
\left.\frac{d \sigma}{d E^{\prime}}\right|_{c o l}
$$

Then

$$
\left.\rho \frac{N_{A}}{A} \frac{d \sigma}{d E^{\prime}}\right|_{c o l}
$$

is the average number of collisions with an energy loss between $E^{\prime}$ and $E^{\prime}+d E^{\prime}$ per unit length in a material with density $\rho\left[\mathrm{g} / \mathrm{cm}^{3}\right]$ and atomic number $A[\mathrm{~g} / \mathrm{mol}] . N_{A}$ $[1 / \mathrm{mol}]$ is Avogadro's number. This leads to the average energy loss per length $d x$ 


$$
-\left.\frac{d E}{d x}\right|_{c o l}=\left.\rho \frac{N_{A}}{A} \int_{E_{\min }}^{E_{\max }} E^{\prime} \frac{d \sigma}{d E^{\prime}}\right|_{c o l} d E^{\prime}=\rho \frac{N_{A}}{A} k_{c o l} .
$$

In literature one often finds the thickness $d x$ measured in $\mathrm{g} / \mathrm{cm}^{2}$ and the energy loss $-\left.\frac{d E}{d x}\right|_{c o l}$ given in $\mathrm{MeV} \mathrm{cm}^{2} / \mathrm{g}$. We will note $-\left.\frac{1}{\rho} \frac{d E}{d x}\right|_{\text {col }}$ instead, which leaves $d x$ with the unit length. Let $k_{c o l(<\nu)}$ represent the energy loss resulting from distant collisions and $k_{\operatorname{col}(>\nu)}$ the energy loss resulting from close collisions, then the total energy loss is given by the sum of the two

$$
-\left.\frac{1}{\rho} \frac{d E}{d x}\right|_{c o l}=\frac{N_{A}}{A}\left(k_{\operatorname{col}(<\nu)}+k_{\operatorname{col}(>\nu)}\right)
$$

\section{Distant Collisions}

For the calculation of the energy loss due to distant collisions $k_{\operatorname{col}(<\nu)}$ it is important to take into account the binding of the electrons to the atoms. The average ionization energy $I[\mathrm{MeV}]$ of the atoms should appear in the formula. Bethe obtained the following result with the help of Born's approximation [16, 17]

$$
k_{c o l(<\nu)}=\frac{C}{\beta^{2}}\left[\ln \frac{2 m_{e} c^{2} \beta^{2} \gamma^{2} \nu}{I^{2}}-\beta^{2}\right] .
$$

Here we have

$C \quad$ - a constant defined by $C=2 \pi Z z^{2} r_{e}^{2} m_{e} c^{2}\left[\mathrm{MeV} \mathrm{cm}^{2}\right]$ and connected to the Particle Data Group's constant $K$ [II] by $C=z^{2} Z K / 2 N_{A}$,

$Z \quad$ - the atomic charge number of the material,

$z \quad$ - the charge of the incident particle in unit charges,

$r_{e} \quad$ - the classical electron radius $r_{e}=e^{2} / 4 \pi \varepsilon_{0} m_{e} c^{2}$,

$m_{e} \quad$ - the electron mass,

$e \quad-$ the electron charge,

$\varepsilon_{0} \quad$ - the dielectric constant of the vacuum,

$c \quad$ - the speed of light,

$\beta \quad$ - the velocity of the particle in units of $c$ and

$\gamma \quad$ - is given by $1 / \sqrt{1-\beta^{2}}$ as usual.

Eq. 1.5 is valid for particles of any kind, with positive or negative charge and with velocity large compared to the velocity of the atomic electrons. 


\section{Close Collisions}

For close collisions we start with an investigation of the maximum transferable energy. As mentioned previously, a close collision of the particle with an atomic electron is not necessarily different from a collision between a charged particle and a free electron. The application of the principles of conservation of energy and momentum leads to the following relation for the maximum kinetic energy, that can be imparted to a free electron in a collision by a particle of mass $m$ and momentum $p$ [1]]

$$
E_{\text {max }}=\frac{2 m_{e} p^{2}}{m^{2}+2 \gamma m_{e} m+m_{e}^{2}}
$$

For very relativistic particles $\left(E_{k i n} \approx E, p c \approx E\right)$ Eq. 1.6 becomes

$$
E_{\max } \approx \frac{E^{2}}{\frac{m^{2} c^{2}}{2 m_{e}}+E}
$$

For example, in a muon-electron collision the maximum transferable energy is $E_{\text {max }} \approx E^{2} /(E+11)$, when the energy of the muon $E$ is measured in $\mathrm{GeV}$. A $200 \mathrm{GeV}$ muon can be practically stopped by a head-on collision with an electron, because in this extreme relativistic case almost the total energy $(\approx 95 \%)$ in transferred to the electron. $\left.E_{\text {max }}\right)$

The energy loss due to close collisions $k_{\operatorname{col}(>\nu)}$ is calculated by integration ( $\nu \ll$

$$
k_{c o l(>\nu)}=\left.\int_{\nu}^{E_{\max }} E^{\prime} \frac{d \sigma}{d E^{\prime}}\right|_{c o l} d E^{\prime} .
$$

The different formulas for the differential collision cross sections for particles with or without spin are given in Appendix A. For particles with Spin 0, mass $m$ larger than the mass of electrons and energy small compared to $E_{c}=m^{2} c^{2} / m_{e}$ (Eq. A.5) we get

$$
k_{\operatorname{col}(>\nu)}=\frac{C}{\beta^{2}}\left[\ln \frac{E_{\max }}{\nu}-\beta^{2}\right]
$$

\section{Total Energy Loss}

The total energy loss for heavy particles is calculated using Eqs. 1.4, 1.5 and 1.9 as 


$$
-\left.\frac{1}{\rho} \frac{d E}{d x}\right|_{c o l}=\frac{C}{\beta^{2}} \frac{N_{A}}{A}\left[\ln \frac{2 m_{e} c^{2} \beta^{2} \gamma^{2} E_{\max }}{I^{2}}-2 \beta^{2}\right]
$$

As expected, this expression is independent of the arbitrary value of $\nu . E_{\max }$ may be substituted from Eq. 1.6.

For electrons and positrons Eq. 1.10 must be modified somewhat for two reasons. One is the small mass of the incident electron/positron; the assumption that the incident particle remains undeflected during the collision process is therefore invalid. The other reason is that for electrons the collisions are between identical particles and we must take into account their indistinguishability. The maximum energy transfer allowed becomes $E_{\max }=E_{k i n} / 2$, where $E_{k i n}$ is the incident electrons kinetic energy. The total energy loss for electrons and positrons is calculated from Eqs. 1.4, 1.5, 1.6, 1.8, A.1] and A.2. With $\beta \approx 1$ one obtains [15]

$$
-\left.\frac{1}{\rho} \frac{d E}{d x}\right|_{c o l}=C \frac{N_{A}}{A}\left[\ln \frac{\pi^{2} \gamma^{3}\left(m_{e} c^{2}\right)^{2}}{I^{2}}-a\right] .
$$

where $a=2.9$ for electrons and $a=3.6$ for positrons.

\section{The Density Effect}

For relativistic particles, the value of the transverse electric field increases with the energy. As a consequence, the distant collision contribution to the total energy loss due to ionization and excitation increases as $\ln (\beta \gamma)$ [1]]. Since materials become polarized, the electric field of the particle is partly screened. This introduces the density effect correction $\delta$. At very high energies $\delta$ becomes

$$
\delta \rightarrow 2 \ln \left(\hbar \omega_{p} / I\right)+2 \ln (\beta \gamma)-1
$$

Here $\hbar \omega_{p}$ is the plasma energy of the medium that is defined by

$$
\hbar \omega_{p}=\sqrt{4 \pi N_{e} r_{e}^{3}} \frac{m_{e} c^{2}}{\alpha}
$$

with the electron density $N_{e}$ and the fine structure constant $\alpha \approx 1 / 137$. Eq. 1.10 becomes 


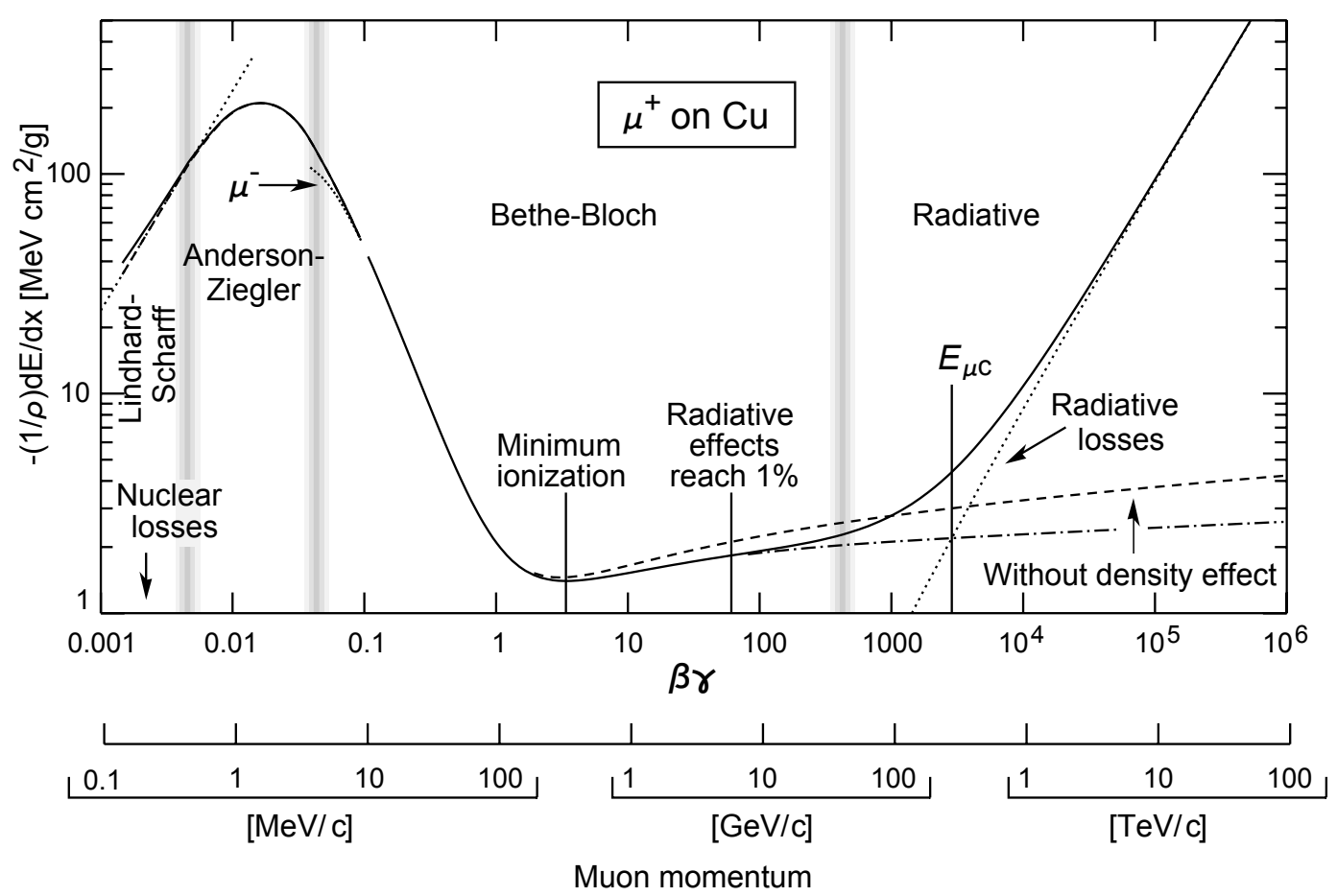

Figure 1.3: The energy loss $-\frac{1}{\rho} \frac{d E}{d x}$ due to ionization and due to bremsstrahlung for positive muons in copper as a function of $\beta \gamma=p_{\mu} / m_{\mu} c$ over nine orders of magnitude in momentum (twelve orders of magnitude in kinetic energy) [11]. For the energy loss due to ionization and excitation the curves with and without density effect correction are shown. The critical energy $E_{\mu C}$, at which the energy loss due to ionization equals the energy loss due to bremsstrahlung is indicated. The solid curve indicates the total energy loss which is the sum of the two.

$$
-\left.\frac{1}{\rho} \frac{d E}{d x}\right|_{c o l}=\frac{C}{\beta^{2}} \frac{N_{A}}{A}\left[\ln \frac{2 m_{e} c^{2} \beta^{2} \gamma^{2} E_{\max }}{I^{2}}-2 \beta^{2}-\delta\right] .
$$

This is the Bethe-Bloch equation for the energy loss due to ionization and excitation for particles heavier than electrons. Fig. 1.3 shows the energy loss due to ionization and excitation of muons in copper versus the muon momentum. The density effect correction becomes important for muon momenta $p_{\mu} \gtrsim 200 \mathrm{MeV} / \mathrm{c}$.

\section{Statistical Fluctuations of the Energy Loss due to Ionization and Excitation}

The quantity $\left(-\left.\frac{d E}{d x}\right|_{c o l}\right) \delta x$ is the average energy loss due to Ionization and Excitation in a layer of the medium with thickness $\delta x$. The real energy loss will fluctuate around 
this average value from event to event. The energy loss distribution is called the Landau distribution [18] and is skewed towards high values (the Landau tail). Only for a thick layer, where the energy loss exceeds one half of the original particle energy, the distribution becomes roughly Gaussian [11]].

\subsubsection{Other Interaction Mechanisms of Radiation with Matter}

The energy loss due to ionization and excitation is not the only interaction process of radiation with matter. Charged particles can also lose energy by radiation (bremsstrahlung). The energy loss connected with the processes of transition radiation and $\breve{C}$ erenkov radiation are negligible, nevertheless they are important processes for identification of charged particles. Moreover, we distinguish three processes in which photons interact with matter: the Photo Electric Effect, the Compton Effect and the Pair Production.

\section{Radiation Loss by Charged Particles}

When the distance of closest approach of a fast charged particle becomes smaller than the atomic radius, the deflection of its trajectory in the electric field of the nucleus becomes the most important effect. Let $\left.\frac{d \sigma(\Theta)}{d \Omega}\right|_{\text {scat }}$ be the differential atomic cross section that a particle of momentum $p$ and velocity $v=\beta c$ undergoes a collision which deflects its trajectory into the solid angle $d \Omega$ at angle $\Theta$ to its original direction of motion. If one neglects both the finite dimension of the nucleus and the shielding of its field by the atomic electrons, one obtains the well-known Rutherford scattering formula [19, 20]

$$
\left.\frac{d \sigma(\Theta)}{d \Omega}\right|_{\text {scat }}=\frac{z^{2} Z^{2} r_{e}^{2}}{4}\left(\frac{m_{e} c}{\beta p}\right)^{2} \frac{1}{\sin ^{4}(\Theta / 2)}
$$

Then the term $\left.\frac{N_{A} \rho}{A} \frac{d \sigma(\Theta)}{d \Omega}\right|_{\text {scat }}$ gives the average number of collisions per length in a medium of density $\rho$ with scattering of the particle into $d \Omega$. The multiple Coulomb scattering distribution is roughly Gaussian for small angles but at larger angles it behaves like Rutherford scattering, having larger tails than does a Gaussian distribution $[11,21,22]$.

In some cases a photon of energy comparable with that of the deflected particle is emitted during the scattering process, e.g.

$$
\mathrm{e}^{-}+\text {nucleus } \rightarrow \mathrm{e}^{-}+\gamma+\text { nucleus }^{\prime}
$$


Radiation phenomena occur at distances of the order of the atomic radius so that the screening of the electric field of the nucleus by the atomic electrons has to be taken into account [23]. However, the field acting on the particle during the deflection process can be considered as the Coulomb field of a point charge $Z e$ at the center of the nucleus [15]. The mean energy loss of an electron due to bremstrahlung is [II]]

$$
-\left.\frac{1}{\rho} \frac{d E}{d x}\right|_{r a d}=\frac{E}{X_{0}}
$$

The characteristic amount of matter traversed is called the radiation length $X_{0}$, measured in $\mathrm{g} / \mathrm{cm}^{2} . x=X_{0} / \rho$ is the mean length of electron trajectory through a medium of density $\rho$, over which the high energy electron loses all but $1 / e$ of its energy by bremsstrahlung. Approximate formulas for $X_{0}$ are given in [19]. The energy loss by radiation depends strongly on the absorbing material. For each material we can define a critical energy $E_{c}$ at which the radiation loss equals the ionization loss. For electrons we find

$$
E_{c}=\frac{610 \mathrm{MeV}}{Z+1.24} \quad \text { for solids and } \quad E_{c}=\frac{710 \mathrm{MeV}}{Z+0.92} \quad \text { for gases. }
$$

Using Eqs. 1.17, the critical energy for copper $(Z=29)$ is $20 \mathrm{MeV}$ and for helium $(Z=2)$ it is $243 \mathrm{MeV}$. Bremstrahlung dominates the energy loss above this energy; ionization dominates at lower energies.

At sufficiently high energies, radiative processes become more important than ionization for all charged particles. The mean energy loss due to bremstrahlung of a charged particle of mass $m$ and charge $z e$ (where $-e$ is the charge of the electron) is found from Eq. 1.16 by scaling with $D=\left(m_{e} / m\right)^{2}$, where $m_{e}$ is the electron mass. The critical energy scales with $1 / D$. For muons in copper the two energy loss mechanisms are compared in Fig. 1.3. The critical energy is around $800 \mathrm{GeV}$.

\section{Čerenkov Radiation}

If the velocity of a particle is larger than the velocity of light in the medium $(v>n c$, $n=$ the refractive index of the material), it emits Črenkov radiation at a characteristic angle $\Theta_{c}$ given by $\cos \Theta_{c}=1 / n \beta$ [1]]. The number of emitted photons with a wavelength $\lambda$ is

$$
\left.\frac{d^{2} N}{d E d x}\right|_{c e r}=\frac{2 \pi \alpha z^{2}}{\lambda^{2}}\left(1-\frac{1}{\beta^{2} n^{2}(\lambda)}\right)
$$


The energy loss connected with this process is negligible but it is used in the detection and identification of particles (electron/pion separation, pion/proton separation and other). Čerenkov counters utilize one or more of the properties of Čerenkov radiation: the existence of a threshold for Čerenkov radiation, the dependence of $\Theta_{c}$ on the velocity $v=\beta c$ of the particle and/or the dependence of the number of emitted photons on the velocity of the particle.

\section{Transition Radiation}

Characteristic transition radiation is used for identifying fast electrons in Transition Radiation Detectors (TRDs; for example, see [24]). Consider a particle of charge ze crossing a boundary between vacuum and a material with a plasma frequency $\hbar \omega_{p}$ given by Eq. 1.13. For typical radiator materials (Styrene) it is about $20 \mathrm{eV}$. The radiated energy is

$$
E_{t r}=\alpha z^{2} \gamma \hbar \frac{\omega_{p}}{3}
$$

The typical emission angle is $1 / \gamma$. Several layers of material lead to several boundaries which increases the radiated energy. The radiated energy increases with $\gamma$. Since electrons are in general the fastest particles observed in an experiment (due to their low mass), TRDs can provide electron/pion separation in the momentum range $0.5 \mathrm{GeV} / \mathrm{c}$ $\lesssim \mathrm{p} \lesssim 100 \mathrm{GeV} / \mathrm{c}[24]$.

\section{Photon Interactions with Matter}

We distinguish three processes in which photons interact with matter:

Photo Electric Effect: The interaction of the photon with the atom as a whole leads to the Photo Electric Effect. The photon is absorbed and an electron is emitted from the atom, e.g.

$$
\gamma+\text { atom } \rightarrow \text { atom }^{+}+\mathrm{e}^{-}
$$

The cross section falls at high energies roughly as $Z^{5} / \hbar \omega$ [19], where $Z$ is the atomic charge number of the absorber material and $\hbar \omega$ is the energy of the photon. The Photo Electric Effect is important up to energies of around $100 \mathrm{keV}$ $(10 \mathrm{MeV})$ for materials like carbon with $Z=6$ (lead with $Z=82$ ).

Compton Scattering: The interaction of the photon with a free electron leads to the Compton Effect. The photon transfers a part of its energy and momentum to the electron initially at rest, e.g. 


$$
\gamma+\mathrm{e} \rightarrow \gamma^{\prime}+\mathrm{e}^{\prime}
$$

The cross section is proportional to $Z / \hbar \omega$ [19]. The Compton effect is important for photon energies from about $100 \mathrm{eV}$ to about $1 \mathrm{GeV}(10 \mathrm{GeV})$ in carbon (lead).

Pair Production: The interaction of the photon with the Coulomb field of the nucleus leads to the phenomenon of Pair Production, whereby the photon disappears and an electron and a positron come into existence simultaneously, e.g.

$$
\gamma+\text { nucleus } \rightarrow \mathrm{e}^{+}+\mathrm{e}^{-}+\text {nucleus }{ }^{\prime}
$$

The Feynman diagram is similar to that of bremsstrahlung, e.g.

$$
\mathrm{e}^{-}+\text {nucleus } \rightarrow \mathrm{e}^{-}+\gamma+\text { nucleus }{ }^{\prime}
$$

The cross sections of the two processes are therefore closely related? The cross section for pair production is proportional to $Z^{2}$. At high energies it becomes independent of the energy of the photon and screening of the electric field of the nucleus by the atomic electrons has to be taken into account. Then the cross section becomes [19]

$$
\sigma_{\text {pair }} \approx \frac{7}{9} \frac{A}{N_{A}} \frac{1}{X_{0}}
$$

At energies above around $100 \mathrm{MeV}(10 \mathrm{MeV})$ for carbon (lead) this effect dominates.

\section{Hadron Interactions with Matter}

The strong interaction plays an important role in the detection of hadrons $(p, \bar{p}, n, \bar{n}$, $\pi^{ \pm}, \mathrm{K}^{ \pm}, \mathrm{K}^{0}$ ), e.g.

$$
\mathrm{p}+\text { nucleus } \rightarrow \pi^{+}+\pi^{-}+\pi^{0}+\ldots+\text { nucleus }^{\prime} .
$$

\footnotetext{
${ }^{2}$ When a high energy electron or photon is incident on a thick absorber, it initiates an electromagnetic cascade or shower, as pair production and bremsstrahlung generate more electrons and photons with lower energy. The electron energies eventually fall below the critical energy. Then they dissipate their energy by ionization and excitation rather than by the generation of more shower particles. These effects are fundamental to the operation of electromagnetic calorimeters.
} 
When the secondary charged pions hit other nuclei, a hadronic cascade develops. Hadronic cascades also have an electromagnetic component from $\pi^{0} \rightarrow \gamma+\gamma$. The total cross section for nucleons has an elastic and inelastic part. The multiplicity grows logarithmically with the energy [19] and the particles are produced in a narrow cone around the forward direction. Hadronic cascades are fundamental to the operation of hadronic calorimeters. Part of the energy of the incident hadron is spent to break up nuclear bonds. This fraction of the energy is invisible in hadron calorimeters. Further energy is lost by escaping particles like neutrinos and muons as a result of hadron decays $\left(\pi^{ \pm} \rightarrow \mu^{ \pm}+\nu\right)$. Since the fraction of lost binding energy and escaping particles fluctuates considerably, the energy resolution of hadron calorimeters is systematically inferior to electromagnetic calorimeters.

\subsubsection{Energy Loss and Particle Detection with RPCs}

The topic of this thesis are Resistive Plate Chambers (RPCs), which are gaseous avalanche detectors. When charged particles traverse the gas gap of an RPC, they lose a fraction of their kinetic energy by excitation and ionization of atoms or gas molecules. The energy loss per unit of path length for particles heavier than electrons is given by the Bethe-Bloch equation (Eq. 1.14). If an atom in the gas is ionized by the inelastic collision of the traversing particle, free charge carriers are deposited close to the position of the encounter. If the atom is not ionized but brought to an excited state, it promptly loses the excitation energy by the emission of a photon or an Auger electron. The photons will be absorbed by Photo Electric Effect as long as their energies are larger than the minimum ionization potential, or they escape. The energy escaping in the form of photons is not detected by a gaseous particle detector like the RPC.

Electrons and highly relativistic charged particles other than electrons also lose energy by bremsstrahlung. As was mentioned previously, this process becomes the main energy loss mechanism, if the energy of the particle is above the critical energy $E_{c}$. However, most of the lost energy disappears in the form of the radiated photons and the RPC does not respond to that energy loss.

This leaves us with the energy loss due to ionization and excitation being the important fundamental mechanism underlying the operation of RPCs. The energy loss due to ionization and excitation is shown for different materials in Fig. 1.4. Primary clusters of free charge carriers (electron-ion pairs) are deposited along the trajectory of the particle. In the gas gap of the RPC they are collected and multiplied by a strong uniform electric field and the propagation of the growing number of charges induces a signal on the read out electrodes. The primary ionization is characterized by the average number of clusters per unit length and by the cluster size distribution. In this thesis we use the simulation program HEED [25] to calculate these parameters. 


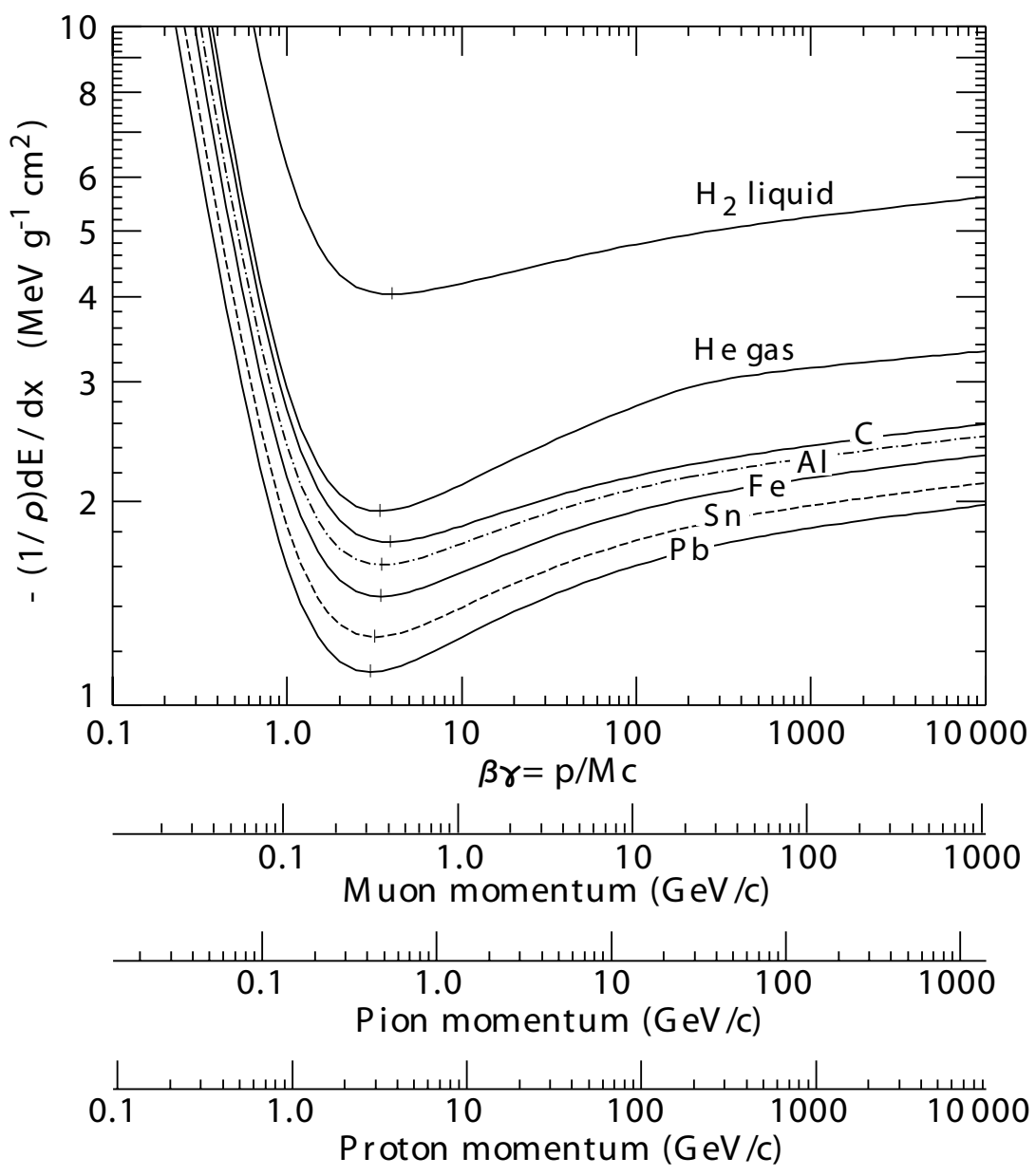

Figure 1.4: The energy loss due to ionization and excitation in liquid hydrogen, gaseous helium, carbon, aluminum, iron, tin and lead [II]]. Radiative effects are not included.

\subsection{Large Area Particle Detectors}

In this section we discuss very briefly the evolution of particle detectors in general and of gaseous parallel plate detectors like the RPC, which is the topic of this thesis, in particular.

The evolution of particle detectors started with the discovery of X-rays and radioactivity in the 1890s. H. Bequerel discovered that the radiation released by uranium salt was capable of blackening photosensitive paper. Also later detectors in nuclear physics based on optical evidencing methods: A scintillating screen was used to detect scattered alpha particles with the eye at the beginning of the 20th century by E. Rutherford and E. Marsden. Later developments used to reconstruct the tracks of charged particles were emulsion techniques and the spark-, cloud-, streamer- and bubble chambers 
in which the particle tracks were photographed. Nevertheless, the technology of particle detectors has with time evolved from these optical methods to electrical methods. Scintillation counters coupled to photo-multipliers are a successful example. Starting in the late nineteen-sixties high voltage operated gaseous detectors such as wire or drift chambers [12] have successfully replaced the scintillation counter in experiments requiring a high spatial resolution. Conversely the scintillator is still a very commonly utilized technique to obtain high time resolution in current apparatus for sub nuclear research.

\subsubsection{Time Resolution}

The wire based gaseous detectors are indeed not competitive with the scintillator as far as time resolution is concerned. The reason lies in the fact that the distance of the closest primary cluster to the wire is exponentially distributed. Due to the $1 / r$ field the amplification is limited to the region around the wire and all electrons need to drift into this region before amplification and the signal generation set in. This introduces a time jitter and limits the time resolution of wire based detectors to a few nanoseconds. A better time resolution is achievable if a strong uniform electric field is used instead of that of a charged wire. Here the avalanche amplification sets in instantly for all primary clusters. The intrinsic detector time resolution is then dominated by the avalanche statistics.

\subsubsection{Spark Counter}

The first gas detector taking advantage of the improved time resolution in strong uniform electric fields was the Keuffel Spark Counter, a gaseous avalanche detector with parallel plate geometry, that was introduced in 1948 [26, 27]. It indeed offered a time resolution (around $1 \mathrm{~ns}$ ) by far better than any of the Geiger-Müller Counters that were commonly used at that time (around $100 \mathrm{ns)} \mathrm{[28].} \mathrm{This} \mathrm{development} \mathrm{opened} \mathrm{the} \mathrm{pos-}$ sibility for the construction of accurate timing systems to measure the velocity of fast charged particles.

Spark Counters generally consist of two planar metal electrodes with a high voltage applied to them. The gap between the plates is filled with a gas. The passage of a charged particle leaves an trail of free charge carriers (primary ionization) in the gas which triggers avalanches of charge carriers in the electric field. At a certain size of the avalanches they transform into a streamer. A streamer is defined as a state where photons contribute to the spread of free charge carriers. At a later stage a conducting plasma filament connecting the two electrodes is formed. Through this channel the electrodes are discharged; a spark is created. The rapidly growing anode current is transformed by a resistor into a fast voltage signal and this signal can be taken as a 
time flag for the arrival of the charged particle. The spark mode of operation leads to large signals that need no further amplification, avoiding electronic time jitter.

A standard spark counter has an area in the order of a few $\mathrm{cm}^{2}$ because as the area increases, the discharge energy in a spark becomes large enough to damage the surface of the counter electrodes. The counting rate of this type of detector is limited by a dead time of typically some milliseconds that is needed to recharge the electrodes.

To overcome these problems, a new type of spark counter introduced resistive plate electrodes and special gas mixtures for photon absorption in 1971 [29, 30]. The resistivity of around $10^{9} \Omega \mathrm{cm}$ of the electrodes leads to a limitation of the discharge to the local area around the primary avalanche and because the high voltage drops only locally, the remaining counter area is still sensitive to particles. The energy in the sparks is much smaller than in the case of metallic electrodes and larger electrode surfaces can be used. The Pestov Spark Counter with a $0.1 \mathrm{~mm}$ gap reaches time resolutions down to $25 \mathrm{ps}$ [31]. However, the very thin gap $(0.1 \mathrm{~mm})$ combined with the high values of the electric field $(500 \mathrm{kV} / \mathrm{cm})$ demand a very good surface smoothness of the electrodes. Moreover, the detector has to be operated at a large overpressure of 12 bar. This ensures a large density of primary ionization in the thin gap to account for a good detection efficiency.

\subsubsection{Parallel Plate Avalanche Chambers}

A Parallel Plate Avalanche Chamber (PPAC) is a single gap gaseous detector very similar to the Spark Counter. However, they are operated in avalanche mode; streamers and discharges are unwanted side effects in this type of detector. It normally consists of two planar electrodes made of metal, or metalized ceramic or plastic, kept apart at a fixed distance of 0.5 to $2 \mathrm{~mm}$ by precise spacers. Its advantages include a fast response and an increased rate capability of up to $10 \mathrm{MHz} / \mathrm{cm}^{2}$ [32]. The time resolution is 100 to $250 \mathrm{ps}$ [33, 34, 35]. Depending on the gas filling, a gain of $10^{3}$ to $10^{4}$ can be reached with a very low discharge probability of $10^{-5}$ for minimum ionizing particles. The PPAC signals are small (about $100 \mathrm{fC}$ on average [33]) which gives a low signalto-noise ratio. To account for a good detection efficiency, the electronics has to be very low-noise and very sensitive, which collides with the fast rise time needed for timing purposes. The possibility of using this technology for large scale applications is questionable. 


\subsubsection{Resistive Plate Chambers}

The Resistive Plate Chamber (RPC) was developed in 1981 by R. Santonico and R. Cardarelli [36, 37]. As the spark counter and the PPAC, the RPC consists of two parallel plate electrodes. At least one of the electrodes is made of a material with high volume resistivity. A charge $Q_{0}$ that enters the resistive electrode surface 'decomposes' with time $t$ following an exponential

$$
Q(t)=Q_{0} e^{-t / \tau} \quad \text { with } \quad \tau=\rho \varepsilon_{0} \varepsilon_{r},
$$

where $\rho$ is the volume resistivity of the material, $\varepsilon_{0}$ is the dielectric constant and $\varepsilon_{r}$ is the relative permittivity of the resistive material. The volume resistivity is connected to the conductivity $\sigma$ by $\rho=1 / \sigma[\Omega \mathrm{cm}]$. Typical glass resistive plates have a volume resistivity of $\rho \approx 10^{12} \Omega \mathrm{cm}$, leading to a 'relaxation time' $\tau \approx 1 \mathrm{~s}$. The volume resistivity of Bakelite is of the order $\rho \approx 10^{10} \Omega \mathrm{cm}$, which gives a 'relaxation time' $\tau \approx 10 \mathrm{~ms}$. The charges in the resistive electrodes cause the high voltage and thus the electric field in the gas gap to drop locally around the initial avalanche or discharge. Here the detector has a blind spot for a time of the order of the relaxation time $\tau$, but the remaining counter area is still sensitive to particles.

Fig. 1.5 shows a schematic image of an example configuration of an RPC [36]. The gas gap is sandwiched between the two resistive electrode plates. These plates are painted with a graphite coating of surface resistivity 200 to $300 \mathrm{k} \Omega / \square$, which is used to distribute the high voltage on the electrodes. The shown configuration utilizes read out strips running along the whole length of the chamber on both sides of the gap, but perpendicular, allowing read out of the $x$ - and $y$-coordinate of the position of a traversing particle. The strips are separated from the graphite coating by an insulating layer.

RPCs may be operated in avalanche mode or in streamer mode (discharge mode). In avalanche mode the release of the primary charge by the incoming ionizing radiation is followed by the propagation and multiplication of the electrons corresponding to a Townsend avalanche. This is shown schematically in Fig. 1.6. At a large gas gain a change occurs in the avalanche dynamics: Then the avalanche charge carriers influence the electric field in the gas gap and hence their own propagation and multiplication (the space charge effect). If the gas gain is further increased, photons can start to contribute to the propagation of the avalanche and streamers appear [38, 39, 40]. At a later stage, a conductive channel can be formed between the two electrodes, through which the local electrode surfaces are discharged. A weak spark may be created. While in avalanche mode RPCs streamers are an unwanted side effect, streamer mode RPCs make use of the large current pulses induced by the streamers which simplifies the read out of the device. Fig. 1.7 shows schematic images of the streamer development in the gas gap. 


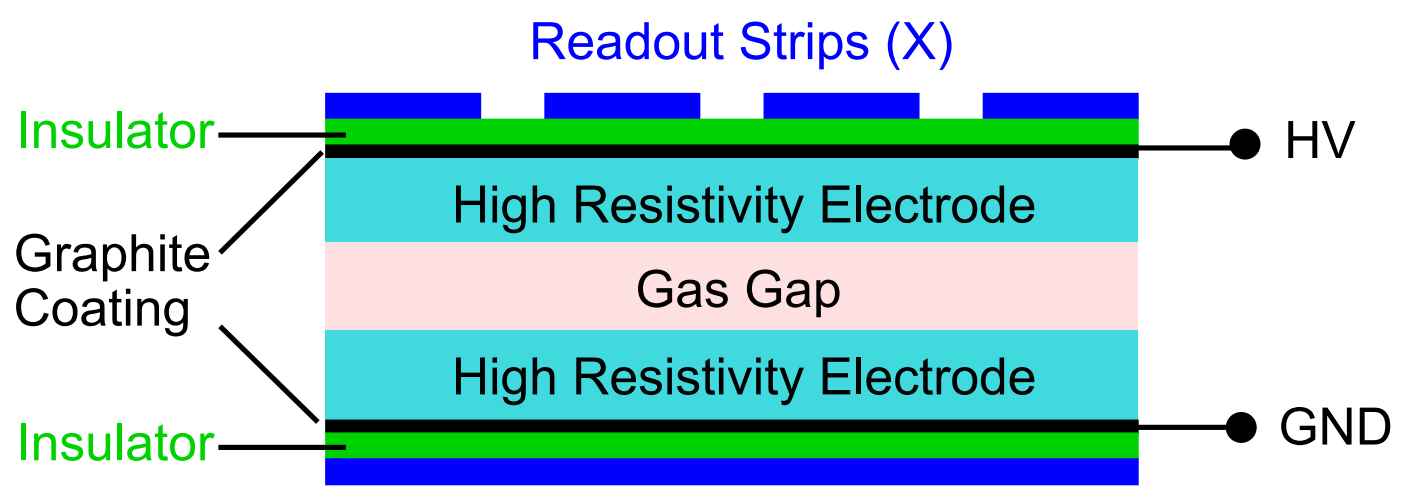

\section{Readout Strips $(Y)$}

Figure 1.5: Schematic image of an RPC geometry as in [36, 37].

a)

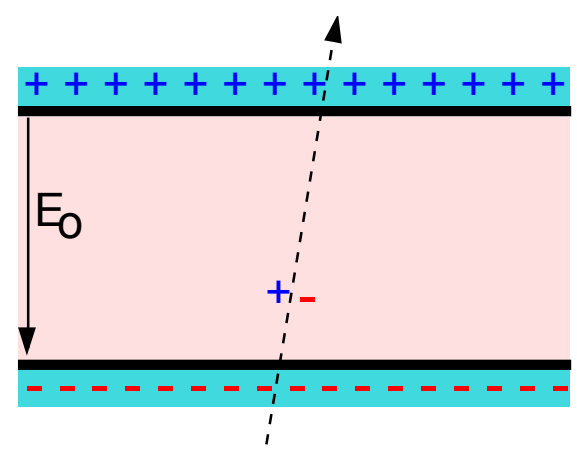

c)

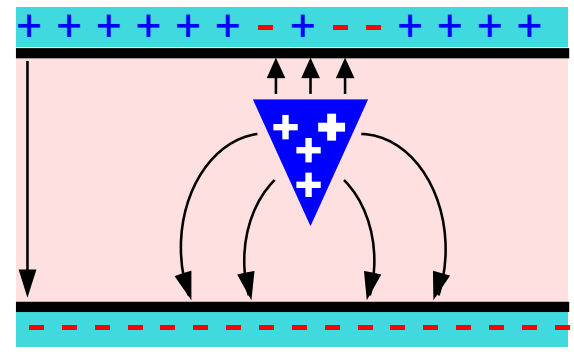

b)

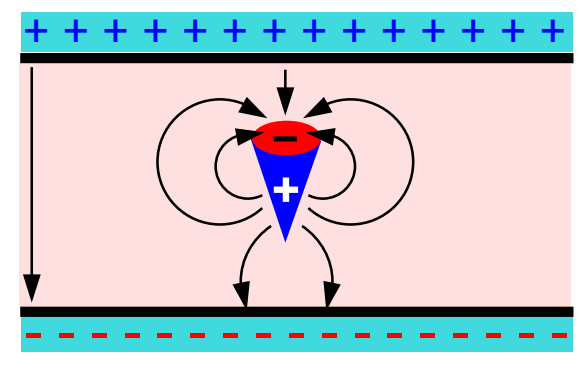

d)

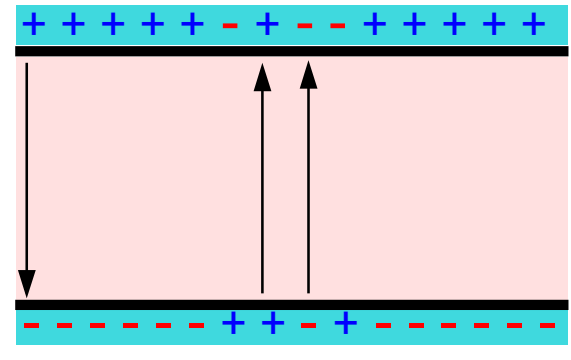

Figure 1.6: A schematic image of the development of an avalanche in an RPC and the electric field deformations caused by the avalanche charges at large gain. $\mathrm{E}_{0}$ is the applied electric field. a) Some gas atoms are ionized by the passage of a charged particle. An avalanche is started. b) The avalanche size is sufficiently large to influence the electric field in the gas gap. c) The electrons reach the anode. The ions drift much slower. d) The ions reach the cathode. The charges in the resistive layers influence the field in a small area around the position where the avalanche developed. 
a)

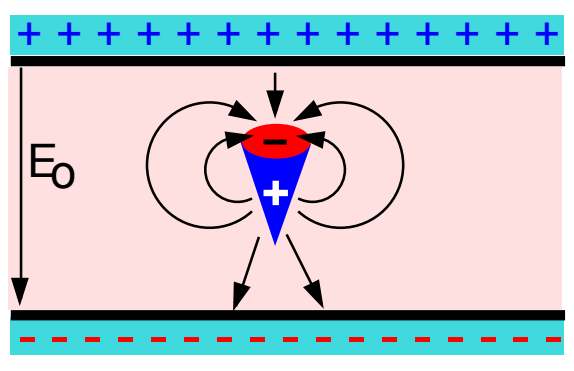

c)

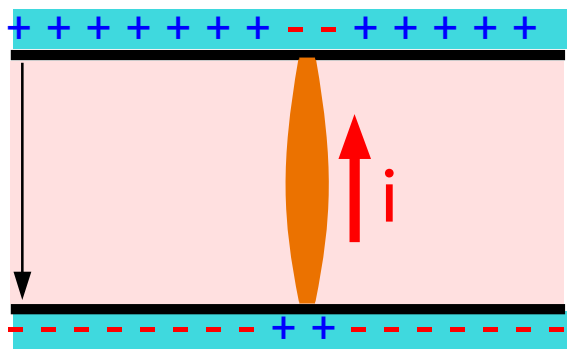

b)

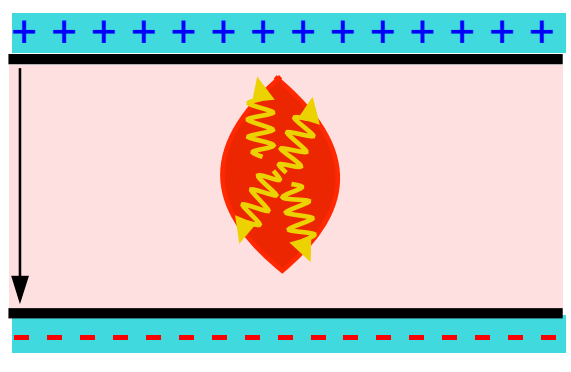

d)

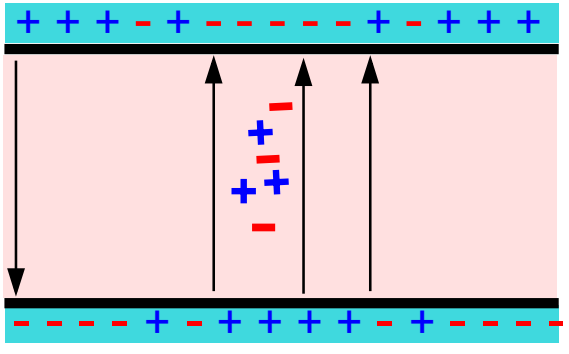

Figure 1.7: A schematic image of the development of a streamer in an RPC. a) An avalanche is developing as in Fig. 1.6. b) The avalanche charges lead to a high field detoriation in the gas gap. Moreover, photons start to contribute to the avalanche development and cause a rapid spread of the avalanche: A streamer evolves. c) A weak spark may be created. The local electrode area is discharged. d) The electric field is strongly decreased around the spot of the avalanche. The detector has a blind spot.

\section{Streamer Mode RPCs}

Single and double gap RPCs operated in streamer mode have so far found application in high energy physics experiments like L3 at CERN [41], BABAR at SLAC [42] and BELLE at KEK [43]. Future applications will include the ARGO experiment at the YangBaJing high altitude cosmic ray laboratory [44] and the OPERA [45] and MONOLITH [46] experiments at LNGS. The muon arm of the ALICE experiment at CERN [47, 48] will also be equipped with streamer mode RPCs.

As the streamer signals are quite large (between $50 \mathrm{pC}$ [49] and a few $\mathrm{nC}$ ([50]), no preamplification is needed and the signals can be discriminated directly. Thus the read out of streamer mode RPCs is quite simple [51, 52]. Double gap chambers operated at electric fields of $40 \mathrm{kV} / \mathrm{cm}$ in streamer mode and with $2 \mathrm{~mm}$ wide gaps reach efficiencies of $99 \%$ and a time resolution around $1 \mathrm{~ns}$. However, the rate capability is limited to a few hundred $\mathrm{Hz} / \mathrm{cm}^{2}$. 


\section{Avalanche Mode RPCs}

The counting rate capability of RPCs is significantly improved if the occurrence of streamers is suppressed and the detector is operated in avalanche mode [53]. This can be achieved by the addition of small contents of $\mathrm{SF}_{6}$ to the gas mixture [39]. RPCs operated in avalanche mode will be used for the muon trigger systems of the ATLAS [54] and CMS [55] experiments at CERN. Multi gap Timing RPCs [56, 57] are implemented in the HARP experiment at CERN [58] and will equip the $176 \mathrm{~m}^{2}$ TOF barell of the ALICE experiment [33].

Because the average pulse charges are a factor ten lower than in streamer mode, the avalanche mode allows to operate this device at a larger particle rate up to a few $\mathrm{kHz} / \mathrm{cm}^{2}$ [49], but it also makes it necessary to introduce low noise electronics. In this thesis we will focus on avalanche mode RPCs. There exist two different designs of RPCs: the Trigger RPC and the Timing RPC. Their performance is described in more detail in the following sections.

\subsection{Trigger RPCs and their Applications}

The Large Hadron Collider (LHC) [60], currently being built at CERN in Geneva, Switzerland, will provide particle physics with the first laboratory tool to access the energy frontier above $1 \mathrm{TeV}$. Protons will be accelerated and stored at $7 \mathrm{TeV}$ in two separate beam pipes, colliding with an unprecedented luminosity of $10^{34} \mathrm{~cm}^{-2} \mathrm{~s}^{-1}$ at $40 \mathrm{MHz}$. In each pipe 2808 counter rotating bunches of approximately $75 \mathrm{~mm}$ length and a radius of about $16 \mu \mathrm{m}$ contain around $10^{11}$ protons each. The superconducting LHC dipoles occupy about $2 / 3$ of the LHC tunnel circumference and provide a dipole field of $8.4 \mathrm{~T}$ strength. An image of the LHC and the positions of the four LHC experiments ATLAS [61], CMS [62], ALICE [63] and LHCb [13] is shown in Fig. 11.8.

High momentum final state muons are amongst the most promising signatures of physics at proton-proton collisions at the LHC. To exploit this potential, the currently built high energy physics experiments ATLAS and CMS will comprise large area muon systems [54, 55] dedicated to detecting the muons. As an example Figs. 1.9 and 1.10 show the ATLAS detector system, that will be taking data starting in 2007 at the LHC.

Resistive Plate Chambers with $2 \mathrm{~mm}$ gap size operated in avalanche mode are used in the ATLAS muon system. The RPCs are implemented on an area of $3650 \mathrm{~m}^{2}$ and with 355.000 independent read out channels to provide information on the presence and arrival time of muons; they are used for triggering on muons. The simultaneous presence of four muons could reveal the decay of the sought after Higgs particle $H$ that might be created in the proton-proton collisions at the LHC. In the high Higgs mass range $m_{H}>130 \mathrm{GeV}$ the so-called 'gold-plated' channel $H \rightarrow Z+Z \rightarrow$ $\mu^{+}+\mu^{-}+\mu^{+}+\mu^{-}$has relatively small background. The LHC bunch crossing interval 


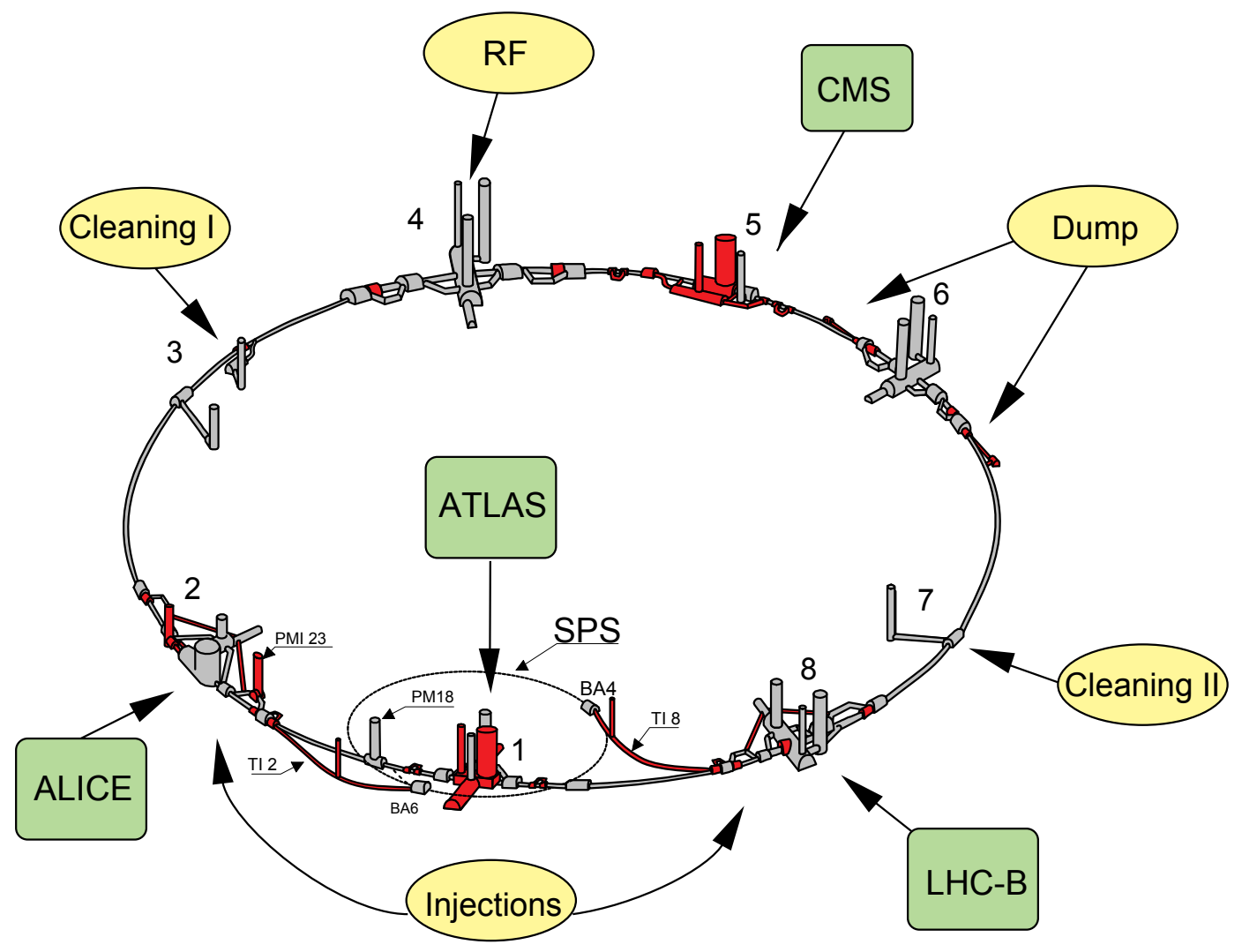

Figure 1.8: A view of the Large Hadron Collider (LHC) and the four LHC experiments [59]. The LHC will be housed in the old LEP tunnel that has $26.659 \mathrm{~m}$ circumference. The two general purpose experiments, ATLAS and CMS, are diametrically opposite in Pits 1 and 5, respectively. The heavy ion experiment ALICE will be in Pit 2. The $\mathrm{LHCb}$ experiment is dedicated to the study of $\mathrm{CP}$ violation and other rare phenomena in the decay of Beauty particles and is situated in Pit 8.

of $25 \mathrm{~ns}$ sets the scale for the required time resolution of the detectors. To be able to reliably tell for each muon from which collision it originates and to limit random coincidences from background hits, the trigger detectors have to reach a time resolution around $1 \mathrm{~ns}$, which can easily be achieved with RPCs. The tracks of the muons are measured by other detectors in the ATLAS muon system, because here a high position resolution is required. One utilizes drift tubes and cathode strip chambers. Together with the toroidal magnet they form the muon spectrometer which makes possible the measurement of the muon momenta.

We will refer to the type of RPC used for triggering in the muon detector systems as the Trigger RPC from now on. A schematic image of a single gap Trigger RPC is shown in Fig. 1.11. A commonly used gas mixture is $\mathrm{C}_{2} \mathrm{~F}_{4} \mathrm{H}_{2} / \mathrm{i}-\mathrm{C}_{4} \mathrm{H}_{10} / \mathrm{SF}_{6}$ $(96.7 \%, 3 \%, 0.3 \%)$. The operating voltage of $10 \mathrm{kV}$ results in an electric field of 


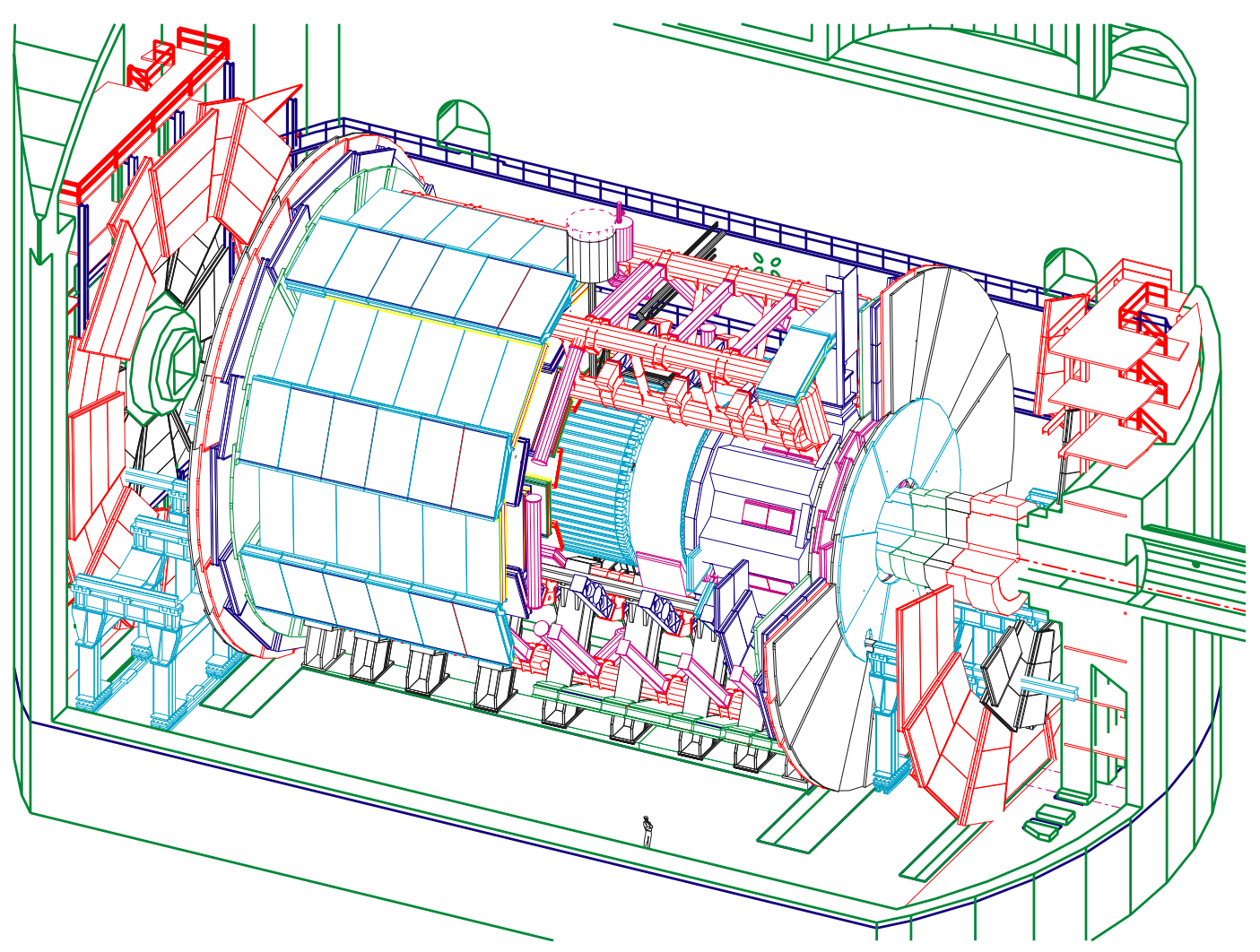

Figure 1.9: View of the ATLAS detector in its underground hall [54]. The muon spectrometer consists of the toroid magnet and the muon chambers in- and outside of the magnet, surrounding the whole detector system. Some muon chambers and parts of the barrel toroid are removed to show the inner structure of the detector.

around $50 \mathrm{kV} / \mathrm{cm}$ in the gas gap(s). The resistive electrodes are made of $2 \mathrm{~mm}$ thick Bakelite plates ${ }^{5}$. Bakelite has a volume resistivity of about $9 \times 10^{9} \Omega \mathrm{cm}$ and a relative permittivity $\varepsilon_{r}=10$. Operated in avalanche mode, double gap Trigger RPCs provide 99\% efficiency and a time resolution of around $1 \mathrm{~ns}$ up to a particle flux of several $\mathrm{kHz} / \mathrm{cm}^{2}$.

\subsection{Timing RPCs and their Applications}

Resistive Plate Chambers with gas gaps of 0.2 to $0.3 \mathrm{~mm}$ are widely used in multi gap configurations [56] for Time-Of-Flight (TOF) purposes [64]. While the performance of multi gap RPCs is comparable to existing scintillator based TOF technology, they feature a significantly lower price per channel. We discuss the implementation of the RPC technology in the TOF system of a high energy physics experiment using

\footnotetext{
${ }^{3}$ Bakelite is a phenol-formaldehyde polymer.
} 


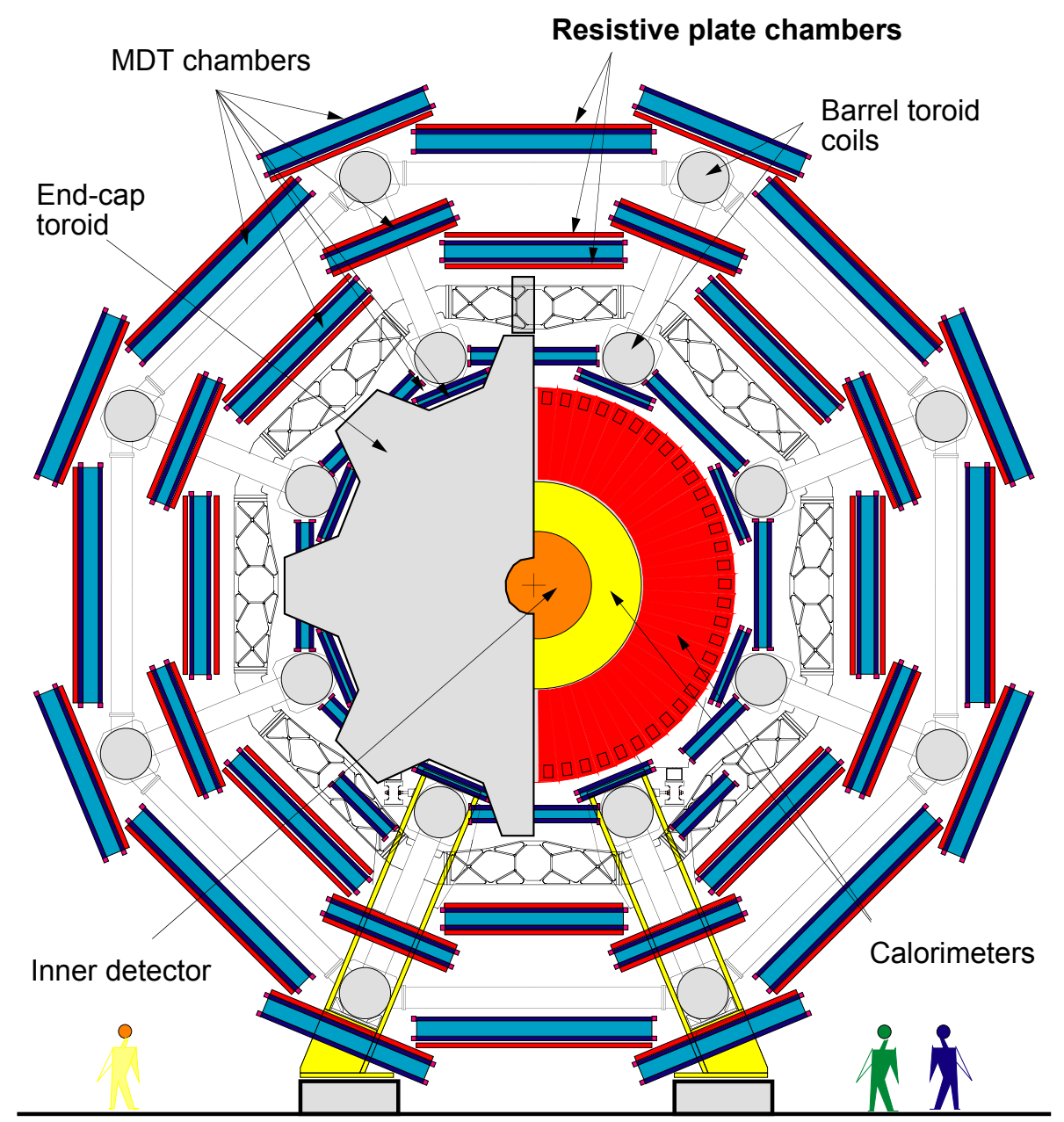

Figure 1.10: Transverse view of the ATLAS muon spectrometer [54]. The position of the Trigger RPCs is indicated.

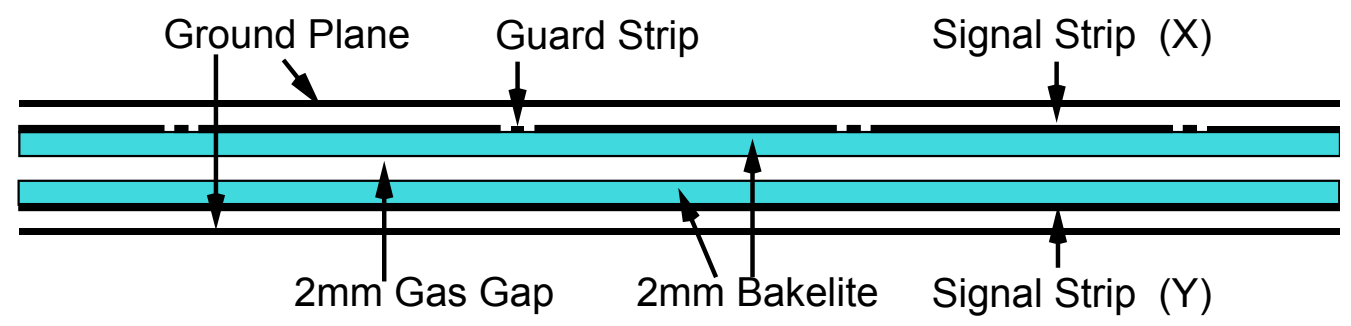

Figure 1.11: Crossection of a single gap Trigger RPC [54, 55]. 


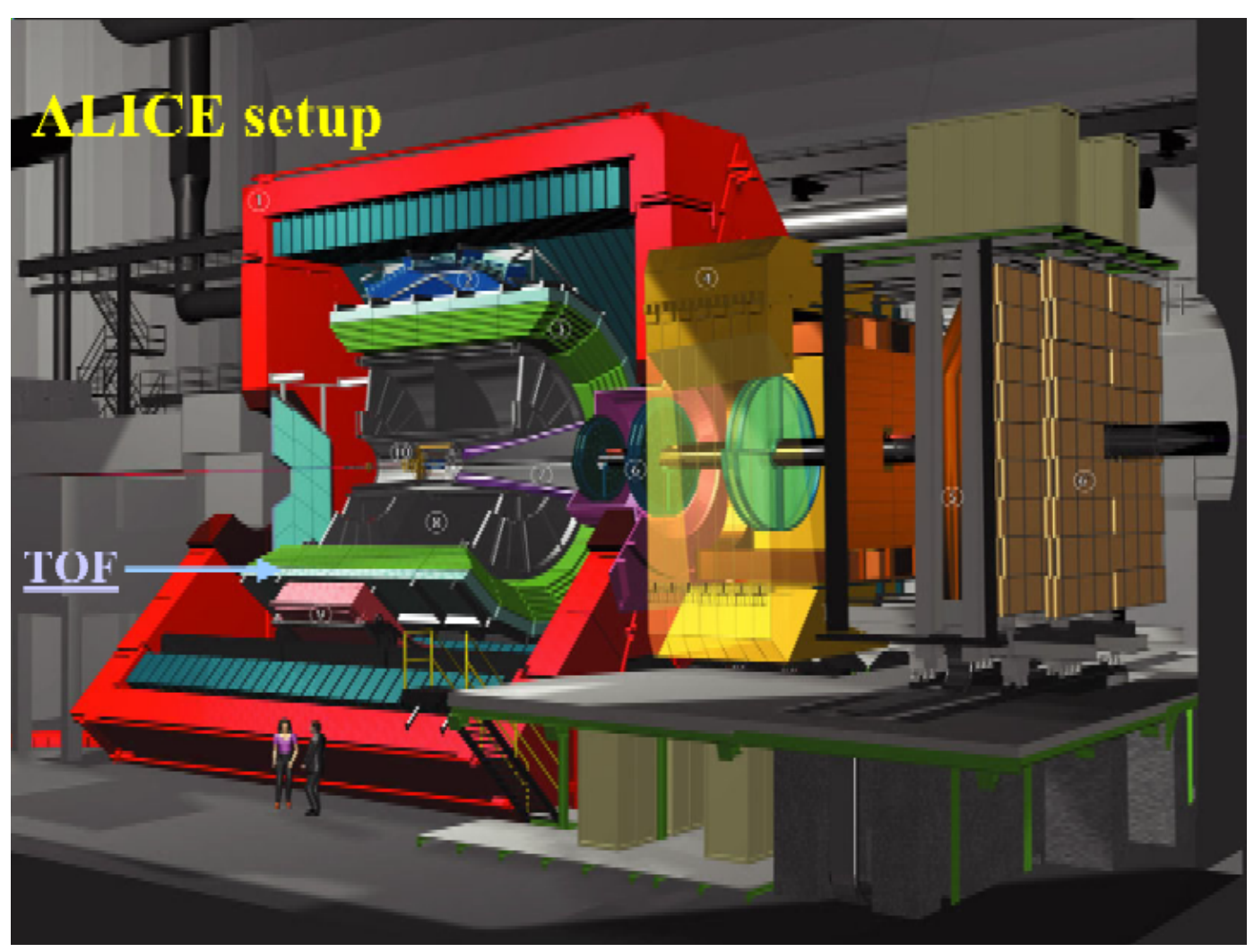

Figure 1.12: A view of the ALICE detector and the TOF subdetector system [33].

the example of the ALICE experiment at CERN (see Fig. 1.12). In the high particle multiplicities of central lead-lead collisions at the Large Hadron Collider, particle identification (PID) is an important design feature. A Time Projection Chamber (TPC) [65] is used as the main tracking system but also provides particle identification by measuring the ionization density which is given by the characteristic energy loss due to ionization $d E / d x$. The upper momentum limit for this kind of particle identification in ALICE is $0.5 \mathrm{GeV} / \mathrm{c}$. Two detector systems are dedicated exclusively to PID: the TOF array is optimized for momenta below $2.5 \mathrm{GeV} / \mathrm{c}[33,66]$ and surrounds the TPC; another smaller system (HMPID [67]) is specialized in higher momenta. With the help of the TOF system particles can be identified by their velocity $v=l / t=\beta c$ (were $t$ is the Time-Of-Flight and $l$ the track length) and the independently measured momentum $p$ as

$$
m_{0}=\frac{p}{\gamma \beta c}=p \sqrt{\frac{t^{2}}{l^{2}}-\frac{1}{c^{2}}} \quad, \quad \gamma=\frac{1}{\sqrt{1-\beta^{2}}}
$$

It turns out that the mass resolution $\partial m_{0} / m_{0}$ is driven much more by the errors 


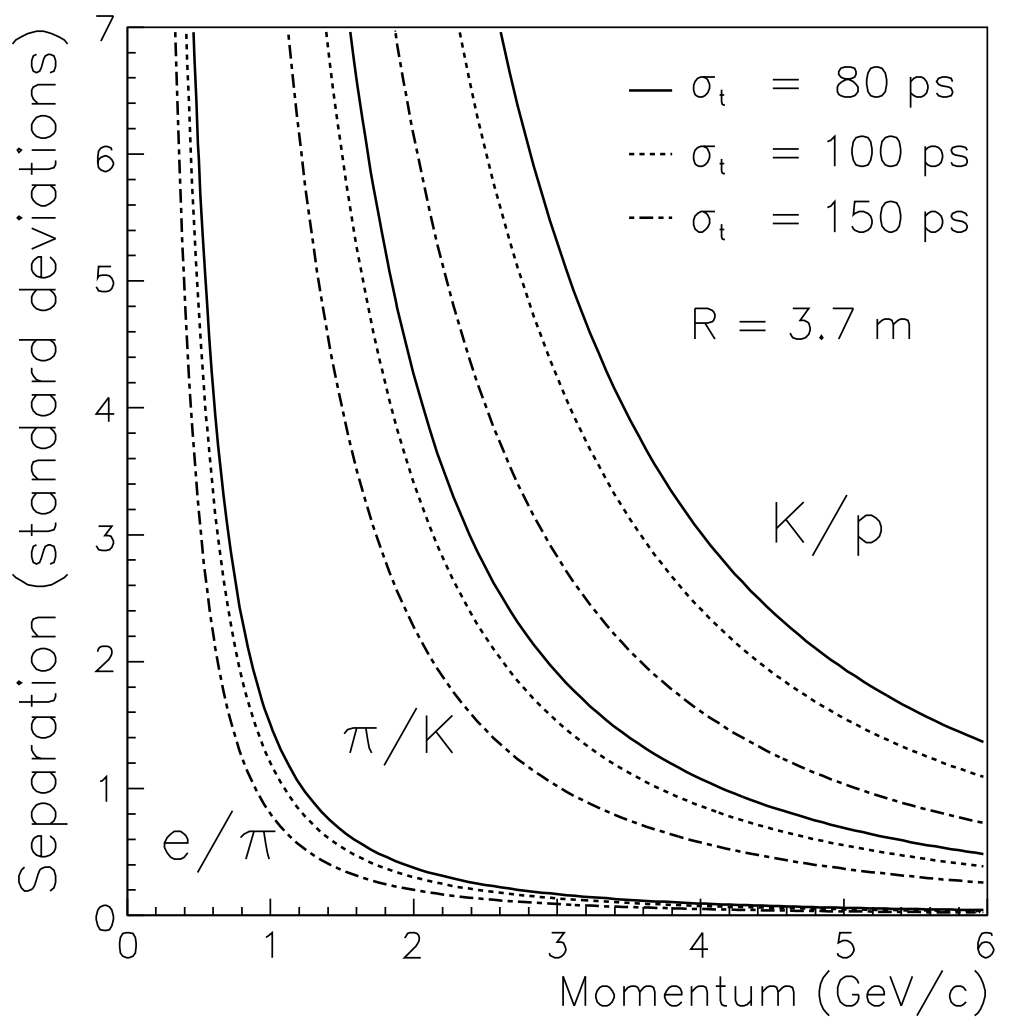

Figure 1.13: The particle separation with a Time-of-Flight detector with a system resolution of 80 to $150 \mathrm{ps}$, located at $R=3.70 \mathrm{~m}$ from the vertex, for particles emitted at an angle perpendicular to the beam axis [33].

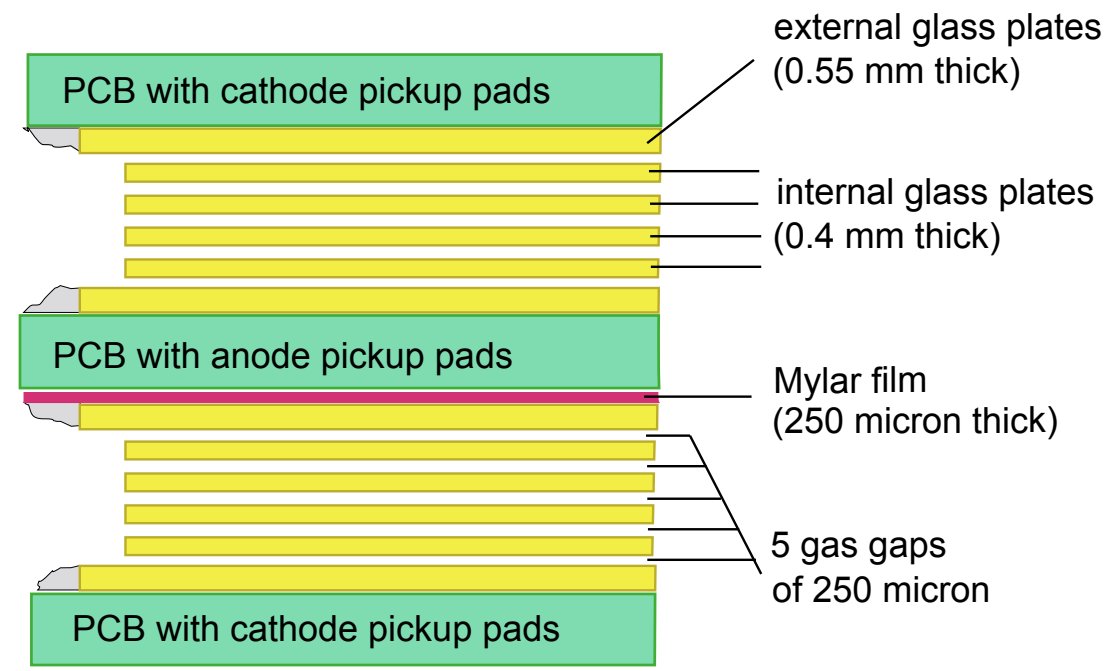

Figure 1.14: A schematic image of the multigap Timing RPCs used in the TOF system of the ALICE experiment [33, 66]. 
on the Time-Of-Flight and track length measurements than on the momentum determination [33]. The technique used for the detectors in the ALICE TOF system should reach an intrinsic time resolution better than around $90 \mathrm{ps.} \mathrm{Including} \mathrm{other} \mathrm{sources} \mathrm{of}$ timing errors, an overall resolution of $150 \mathrm{ps}$ is expected. The nominal performance of a TOF array with a system resolution from 80 to $150 \mathrm{ps}$, located at $R=3.70 \mathrm{~m}$ from the vertex, is shown in Fig. 1.13 for particles emitted at 90 degrees to the beam axis. An overall time resolution of 150 ps guarantees a separation of kaons from pions within three standard deviations up to a momentum of around $1.7 \mathrm{GeV} / \mathrm{c}$. The choice of technology for the ALICE TOF are multi gap RPCs made from glass resistive plates with gap sizes of $0.25 \mathrm{~mm}$ and operated in avalanche mode [33, 66] (see Fig. 11.14). The electric field in the gas gaps is around $100 \mathrm{kV} / \mathrm{cm}$ and the gas mixture is $\mathrm{C}_{2} \mathrm{~F}_{4} \mathrm{H}_{2} /$ i- $\mathrm{C}_{4} \mathrm{H}_{10} / \mathrm{SF}_{6}(90 \%, 5 \%, 5 \%)$. The ALICE TOF system consists of RPCs on an area of $176 \mathrm{~m}^{2}$ with 160.000 individual read out cells of $3 \times 3 \mathrm{~cm}^{2}$.

In a similar way RPCs are implemented in the HARP TOF detector system around a TPC [68]. From now on we refer to this type of Resistive Plate Chamber as the Timing $R P C$. In general, the multi gap Timing RPC technology reaches $99 \%$ efficiency and time resolutions down to $50 \mathrm{ps}[64,68,69,70]$.

\subsection{Summary}

Present and future high energy physics experiments are complex systems that are built of many layers of particle detectors. The task of the detector system as a whole is to identify and to measure the momenta and/or energies of eight different particles: electrons, muons, photons, charged pions, charged kaons, neutral kaons, protons and neutrons. Each particle type leaves its own signature in the detector. For the sub detector systems different technologies are used, but they all rely on the same fundamental physics: the interaction of radiation with matter. For the operation of Resistive Plate Chambers (RPCs), that are the topic of this thesis, the primary ionization in the gas gap due to collisions of the charged particle with the gas atoms is the important mechanism. The free charge carriers that are deposited in the gas gap trigger avalanches of electrons in the externally applied electric field. The propagation of the growing number of electrons induces a current on external strip electrodes.

RPCs are gaseous parallel plate avalanche detectors with electrodes that are made of a material with high volume resistivity. This ensures that possible discharges are localized and do not affect the entire detector. RPCs are widely used as large area particle detectors in certain subsystems of present and future experiments, where a good time resolution is needed.

One example for the implementation of RPCs in high energy physics experiments is the muon system of the ATLAS experiment at the Large Hadron Collider (LHC) at CERN. Here RPCs are implemented on $3650 \mathrm{~m}^{2}$ with 355.000 read out channels 
as trigger detectors that provide information on the presence of muons. Single gap Trigger RPCs with $2 \mathrm{~mm}$ gas gaps, Bakelite resistive plates and an applied electric field strength of $50 \mathrm{kV} / \mathrm{cm}$ are used and provide above $98 \%$ efficiency and around $1 \mathrm{~ns}$ time resolution. The good time resolution is needed to provide bunch crossing identification for the $40 \mathrm{MHz}$ proton-proton collision rate at the LHC.

Another example is the ALICE experiment that will also operate at the LHC. It will investigate lead-lead collisions that result in high multiplicities of secondary particles, thus the challenge here is the particle identification. One technology that will be used is to measure the Time-Of-Flight $t$ over a distance $l$ and to combine it with a separate measurement of the momentum $p$, so that the particle can be identified by its rest mass $m_{0}=p t / l$. Multi gap Timing RPCs with $0.25 \mathrm{~mm}$ gap width and glass resistive plates will be used in the ALICE Time-Of-Flight system on an area of $176 \mathrm{~m}^{2}$ with 160.000 individual read out cells. The applied high voltage leads to an electric field of around $100 \mathrm{kV} / \mathrm{cm}$ in the gas gaps. The detectors reach efficiencies of $99 \%$ and time resolutions of better than 90 ps. 


\section{Chapter 2}

\section{Detector Physics of RPCs}

The topics of this chapter are the basic detector physics and the working principles of Resistive Plate Chambers (RPCs). Attempts at detailed discussions of this topic have been made before [71, 8, 72, 73, 9]. The model suggested in [71] describes the basic processes taking place in RPCs operated in avalanche mode and reproduces some available experimental data. It explains quite well most results but however uses a model for the statistical fluctuation (the Polya Distribution) that is not applicable to RPCs, since it neglects the effect of attachment and it also assumes an unphysical parameter that lacks any clear interpretation. Moreover, a measured mean free path for ionizing collisions is used [74], that is contradicted by other measurements [75] and calculations with the commonly used simulation tool HEED [25]. In [8] this model is extended by a saturation effect that is implemented in a crude way by cutting off the avalanche growth at a certain size. In [72, 73] a simple model is introduced, in which the saturated growth is explained by a constant-coefficient, non-linear differential equation, connected to the logistic function, which was originally introduced to describe the evolution of a biological population in a limited resources environment. In [9] a space charge effect is included by introducing a functional dependence of the effective Townsend coefficient $\alpha_{e f f}$, that describes the average avalanche multiplication $\left(n(z)=e^{\alpha_{e f f} z}\right)$, on the avalanche size. The author also assumes the mean free path for ionizing collisions from [74].

A much more accurate approach involves the dynamic calculation of the electric field contributed by the avalanche charges. We follow this approach and describe the detector physics of the RPC using only well-defined fundamental physics parameters. We use analytic formulas for the potential of a point charge in a three layer geometry like the RPC. With the calculated values for the electric field of the space charge, we further calculate the actual values of the parameters that define the avalanche propagation: the drift velocity, the Townsend and attachment coefficients and the diffusion coefficients. This approach ensures an understanding and description of the evolution of avalanches in a much more elementary way. For the mentioned gas parameters and for the mean free path we use the values that are predicted by the simulation programs 


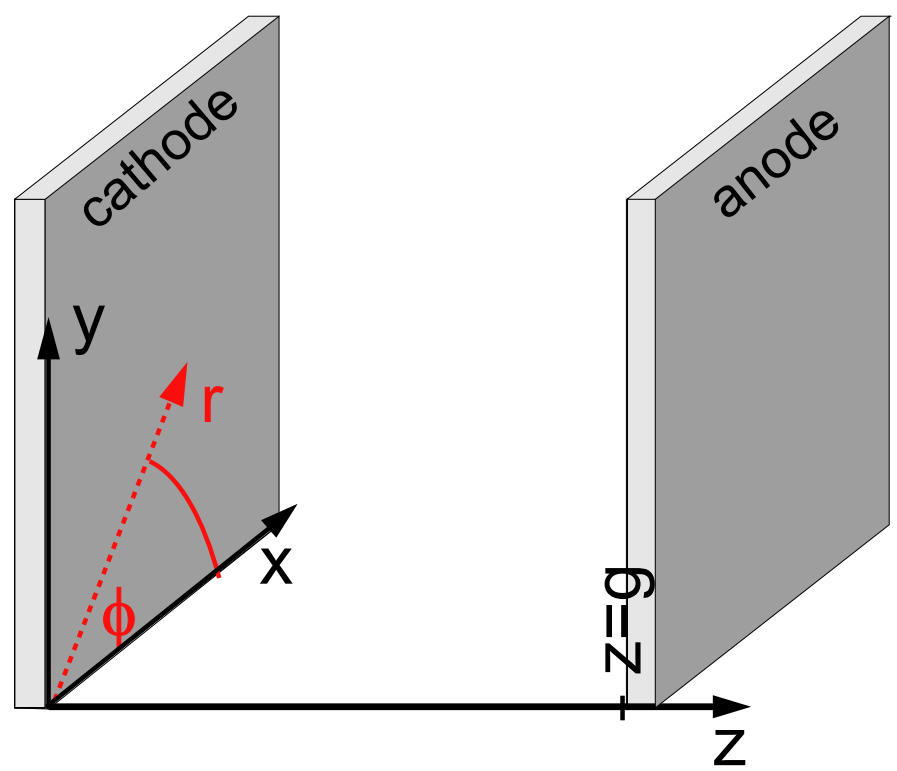

Figure 2.1: In our studies we will mainly use cylindrical coordinates $z, r$ and $\phi$, where the $z$-axis is perpendicular to the cathode and anode, that are situated at $z=0$ and $z=g$.

MAGBOLTZ [76], IMONTE [77] and HEED [25].

We start with a discussion of primary ionization processes (section 2.1), followed by diffusion, drift and the multiplication of electrons under the influence of an electric field (section 2.2). In section 2.3 we investigate the electrostatics of a three layer geometry like an RPC. There we present the analytic formulas that can be used to calculate the electric field contributions of the space charge. The signal generation process and the weighting field formalism are the topic of section 2.4 and finally we shortly discuss the phenomenon of streamers in section 2.5. Based on the knowledge summarized in this chapter we shall present Monte-Carlo simulation models for avalanches in RPCs in chapter 3 .

\subsection{Gas Ionization by Fast Charged Particles}

In the following sections we discuss the average distances between primary clusters, the effect on the detection efficiency of RPCs and the distribution of the number of released electrons per cluster. 


\subsubsection{Distance between Primary Clusters}

We assume that the probability of an ionizing collision does not depend on the previous collision, which is correct if the energy loss is negligible compared to the particle energy. In that case the distance between the ionizing collisions (the distance between primary clusters) is exponentially distributed like

$$
P(z)=\frac{1}{\lambda} \exp \left(-\frac{z}{\lambda}\right)
$$

If $\sigma_{p}(\beta)\left[\mathrm{cm}^{2}\right]$ is the ionization cross section in a gas with density $\rho$, then the mean free path $\lambda$ is given by

$$
\lambda=\frac{A}{\rho N_{A}} \frac{1}{\sigma_{p}(\beta)}
$$

where $A$ is the atomic mass number of the gas $[\mathrm{g} / \mathrm{mol}]$ and $N_{A}$ is Avogadro's number [ $1 / \mathrm{mol}]$. The ionization cross section of a particle with charge $z$ in unit charges and velocity $v=\beta c$, where $c$ is the speed of light, for different gases can be written as [75, 15]

$$
\sigma_{p}(\beta)=4 \pi\left(\frac{\hbar}{m c}\right)^{2} z^{2}\left(M^{2} x_{1}+C x_{2}\right)
$$

where $4 \pi(\hbar / m c)^{2}=1.874 \times 10^{-20} \mathrm{~cm}^{2}, M^{2}$ and $C$ are constants characteristic to the gas and

$$
x_{1}=\frac{1}{\beta^{2}} \ln \left(\beta^{2} \gamma^{2}\right)-1 \quad, \quad x_{2}=\frac{1}{\beta^{2}} \quad, \quad \gamma=\frac{1}{\sqrt{1-\beta^{2}}}
$$

As mentioned previously, the average distance between the clusters $\lambda$ can be obtained using the simulation program HEED [25]. HEED is a Monte-Carlo model based on the photo-absorption ionization model by W.W.M. Allison and J.H. Cobb [78]. HEED data for two typical RPC gas mixtures and for pure isobutane and pure methane are shown in Fig. 2.2. The comparison of measurements for isobutane and methane shows good agreement of simulated and measured data. The measurements are from [75], where we find $M^{2}=14.19$ and $C=141.9$ for isobutane and $M^{2}=4.23$ (3.69) and $C=41.85$ (43.88) for methane, obtained with two different experimental methods. 


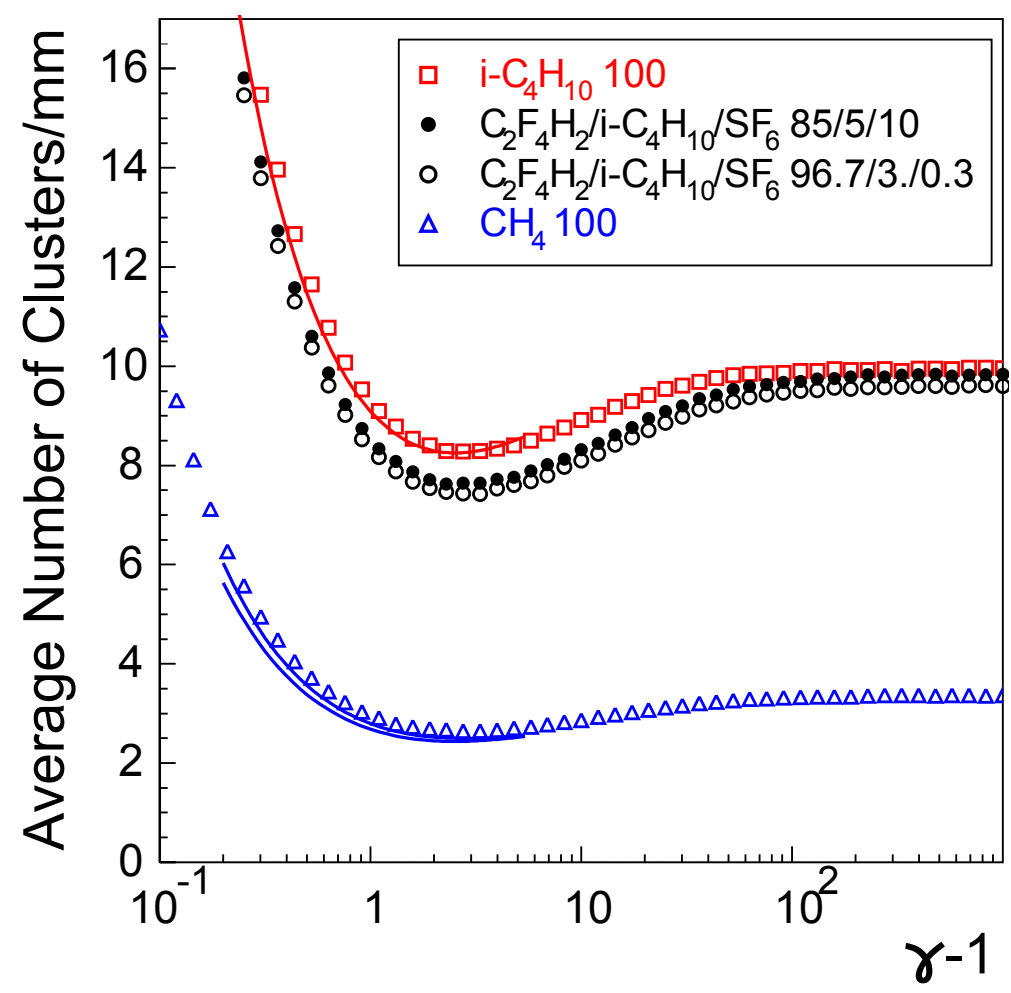

Figure 2.2: The average number of ionizing collisions (clusters) per $\mathrm{mm}(\bar{n}=1 / \lambda)$ as a function of $\gamma-1$ for different gases as predicted by HEED [25]. $T=296.15 \mathrm{~K}$ and $p=1013$ mbar. The solid lines are measurements taken from [75].

\subsubsection{Maximum Detection Efficiency}

Since the distance between the ionizing collisions is exponentially distributed, the number of clusters on a distance $g$ follows a Poisson distribution with an average of $\bar{n}=g / \lambda$. The probability to have $n$ clusters is given by

$$
P(n)=\frac{1}{n !}\left(\frac{g}{\lambda}\right)^{n} e^{-\frac{g}{\lambda}}
$$

The average number of clusters is very different for different gas mixtures. In Table 2.1 we list values of $\bar{n}=g / \lambda$ for $g=1 \mathrm{~mm}$ for a few common gases.

We assume that all primary clusters in the gas gap are detected, which can only theoretically be achieved by either an infinite gas gain or a threshold of zero applied to the signals. With Eq. 2.5 we calculate the maximum detection efficiency $\epsilon_{\max }$ to be

$$
\epsilon_{\max }=1-e^{-\bar{n}}
$$




\begin{tabular}{|c||c|c|c|c|}
\hline Gas & $\mathrm{He}$ & $\mathrm{Ar}$ & $\mathrm{Xe}$ & $\mathrm{i}-\mathrm{C}_{4} \mathrm{H}_{10}$ \\
\hline $\bar{n}$ [clusters $/ \mathrm{mm}$ ] & 0.42 & 2.3 & 4.4 & 8.4 \\
\hline
\end{tabular}

Table 2.1: Simulated values for the average number of ionizing collisions per $\mathrm{mm}$ in four different gases [79]. We assume a minimum ionizing particle.

\begin{tabular}{|c||c|c|}
\hline Gas & $\mathrm{g}[\mathrm{mm}]$ & $\epsilon_{\max }[\%]$ \\
\hline $\mathrm{He}$ & 0.3 & 12 \\
& 2.0 & 57 \\
\hline $\mathrm{i}-\mathrm{C}_{4} \mathrm{H}_{10}$ & 0.3 & 92 \\
& 2.0 & 100 \\
\hline
\end{tabular}

Table 2.2: The maximum detection efficiency for two different typical gap sizes and two different gases.

where $P(0)=e^{-\bar{n}}$ is the probability to find no primary cluster between $z=0$ and $z=g$. In Table 2.2 we compare gaps of different width for a detector filled with two different gases. We find that the maximum detection efficiency depends strongly on the gap width $g$ and on the gas.

\subsubsection{Cluster Size Distribution}

The number of emitted electrons per cluster depends on the amount of energy exchanged at the encounter, which can fluctuate considerably. The distribution is called the cluster size distribution. A method for the calculation of cluster size distributions in argon, based on detailed elastic and inelastic cross sections for low energy electrons, was developed in [80]. We use HEED to calculate the cluster size distribution for the gas mixtures typically used in RPCs. The simulated data for $7 \mathrm{GeV}$ pions and the Timing RPC gas mixtures and for $120 \mathrm{GeV}$ muons and a Trigger RPC gas mixture is shown in Fig. 2.3.

\subsection{Electron Drift and Multiplication}

In this section we discuss the propagation and multiplication of electrons under the influence of an electric field in a gas. 


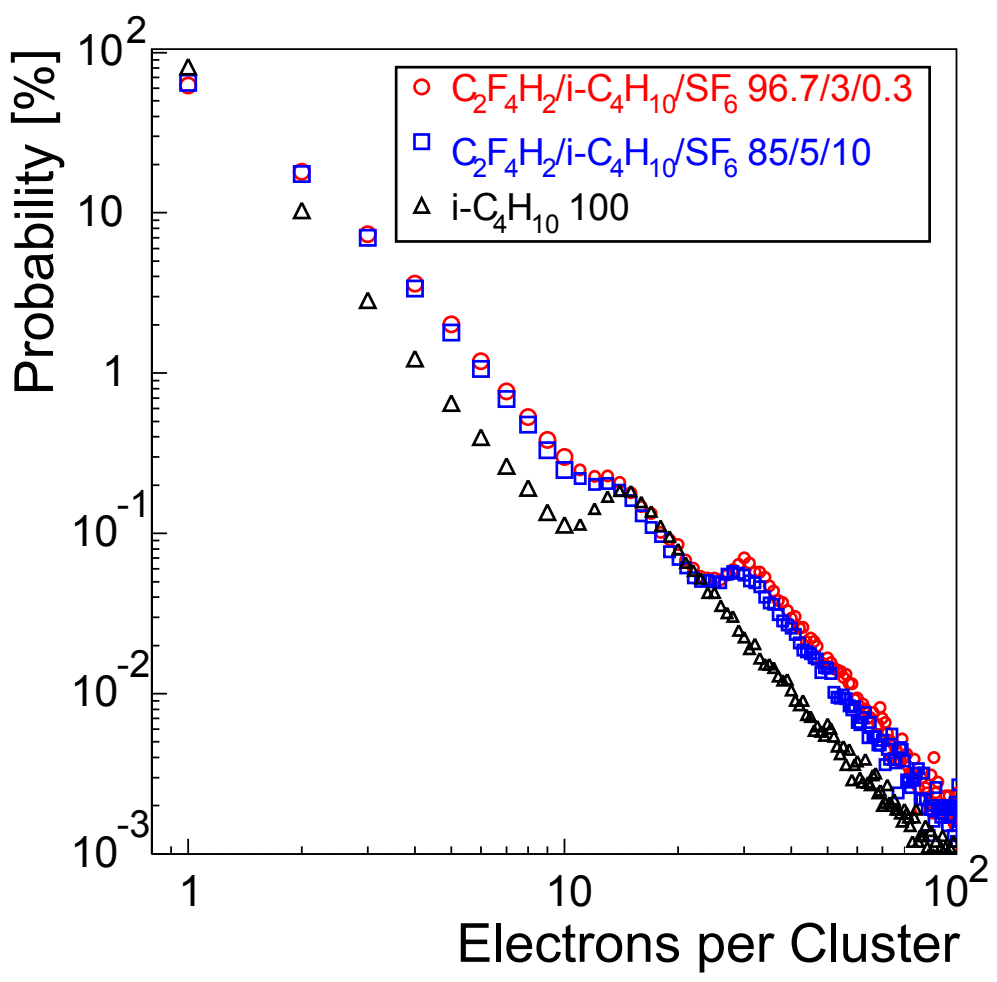

Figure 2.3: Cluster size distributions for two typical RPC gas mixtures and for pure isobutane as calculated with HEED. The incident particle is a $7 \mathrm{GeV}$ pion for isobutane and for the $10 \% \mathrm{SF}_{6}$ mixture and a $120 \mathrm{GeV}$ muon for the $0.3 \% \mathrm{SF}_{6}$ mixture. The temperature of the gas is $T=296.15 \mathrm{~K}$ and the pressure $p=1013 \mathrm{mbar}$. Cutting at 500 electrons the average number of electrons per cluster is 1.9 for isobutane, 2.6 for the $10 \% \mathrm{SF}_{6}$ mixture and 2.8 for the $0.3 \% \mathrm{SF}_{6}$ mixture.

\subsubsection{Thermal Motion and Diffusion}

The diffusion of an electron cloud in a gas is caused by random collisions with the gas atoms due to the thermal motion. A free electron in a gas will assume an energy following a Maxwell-Boltzmann distribution around the mean $\langle E\rangle=3 / 2 k T \approx 40 \mathrm{meV}$, where $k$ is the Boltzmann constant and $T$ the temperature in Kelvin. In case of absence of an external electric field, the diffusion is isotropic and can be described by a Gaussian distribution. A cloud of electrons that is point-like at position $\vec{r}_{0}$ at time $t=0$ will assume the following density distribution after some time $t$ :

$$
\varphi_{i s o t r}(\vec{r}, t)=\frac{1}{(\sqrt{2 \pi} \sigma(t))^{3}} \exp \left(-\frac{\left(\vec{r}-\vec{r}_{0}\right)^{2}}{2 \sigma(t)^{2}}\right)
$$

The sigma of the Gaussian is increasing with time like $\sigma=\sqrt{2 \bar{D} t}$ where $\bar{D}$ 


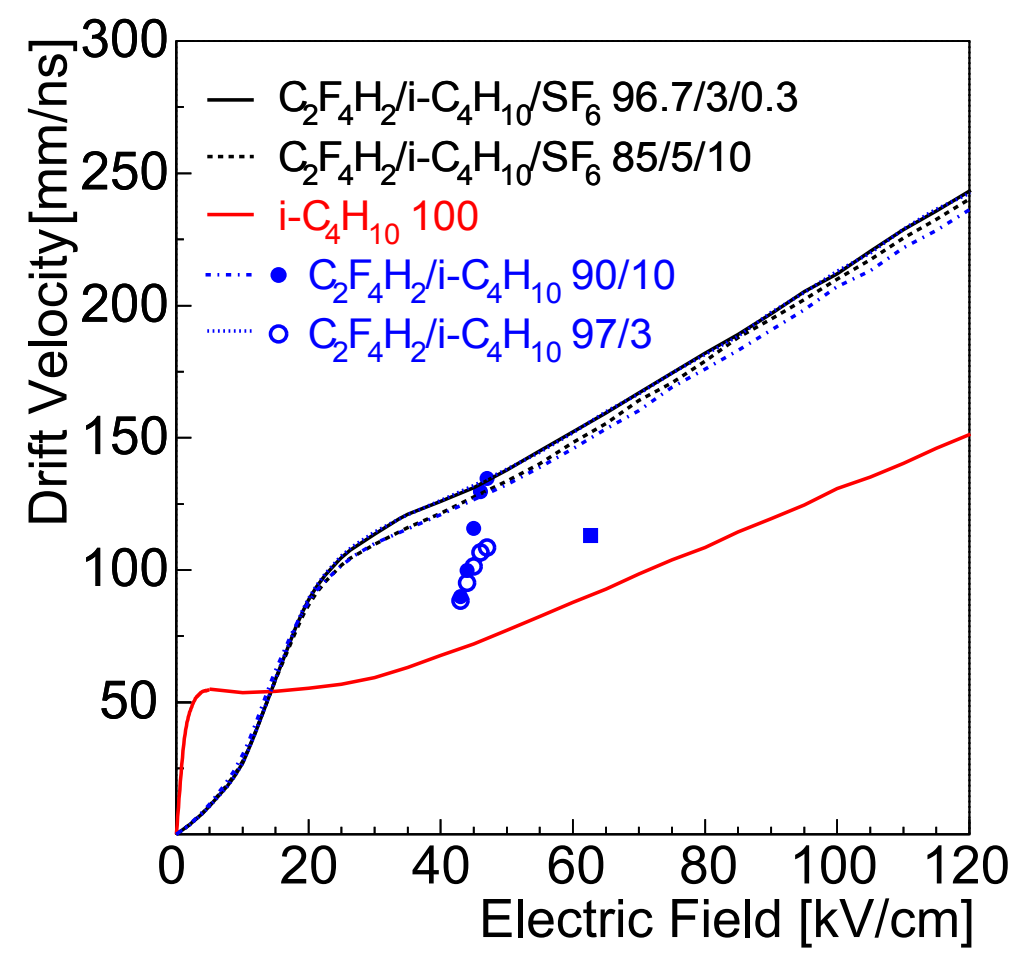

Figure 2.4: The drift velocity calculated with MAGBOLTZ [76] for $\mathrm{C}_{2} \mathrm{~F}_{4} \mathrm{H}_{2} / \mathrm{i}$ $\mathrm{C}_{4} \mathrm{H}_{10} / \mathrm{SF}_{6}(96.730 .3 \%)$ and $(85 \%, 5 \%, 10 \%)$ and for pure isobutane. The temperature of the gas is $T=296.15 \mathrm{~K}$ and the pressure $p=1013 \mathrm{mbar}$. The circles show measurements from [81] for two different mixtures, the square shows a measurement from [82] for $\mathrm{C}_{2} \mathrm{~F}_{4} \mathrm{H}_{2} / \mathrm{i}-\mathrm{C}_{4} \mathrm{H}_{1} 0 / \mathrm{SF}_{6}(96.9 \%, 3 \%, 0.1 \%)$.

$\left[\mathrm{cm}^{2} / \mathrm{ns}\right]$ is a diffusion coefficient.

\subsubsection{Electron Motion due to an Electric Field}

If an electric field is present the diffusion motion is superposed by a constant drift motion due to the electric field. In absence of a magnetic field the drift velocity vector is always in the direction of the electric field lines. On the microscopic level an electron gains the kinetic energy $T=e_{0}|\vec{E}| \delta z$ on a drift distance $\delta z$ between two collisions with gas molecules. Here $e_{0}$ is unit charge and $|\vec{E}|$ is the electric field strength sensed by the electron. In the next encounter some kinetic energy is lost through recoil or excitation and the electron is slowed down. Then it is again accelerated by the electric field and again collides, and so on. On the macroscopic level, averaging over a large number of collisions, one measures an average velocity $v_{D}$. The drift velocity is a function of $E / p$, where $E$ is the electric field sensed by the electrons and $p$ is the gas pressure. This functionality can be calculated with the Monte-Carlo simulation pro- 
gram MAGBOLTZ [76]. A plot for typical RPC gas mixtures and for pure isobutane is shown in Fig. 2.4.

In an electric field the diffusion becomes anisotropic; we have to distinguish longitudinal and transverse diffusion. We assume rotational symmetry and use cylindrical coordinates. Then Eq. 2.7 becomes

$$
\varphi_{i s o t r}(r, z, t)=\frac{1}{\sqrt{2 \pi} \sigma_{L} \sigma_{T}^{2}} \exp \left(-\frac{\left(z-z_{0}\right)^{2}}{2 \sigma_{L}^{2}}-\frac{\left(r-r_{0}\right)^{2}}{2 \sigma_{T}^{2}}\right) .
$$

$z_{0}$ and $r_{0}$ indicate the position of the center of mass of the distribution. Note that here a $\phi$-integration was carried out, leading to an additional factor of $2 \pi$. We introduce two new diffusion coefficients $D_{T}$ and $D_{L}[\sqrt{\mathrm{cm}}]$. They describe the dependence of the width of the Gaussian on the drifted distance $l$. Assuming a constant drift velocity $v_{D}=l / t$ we write $\sigma_{L, T}=\sqrt{2 \bar{D}_{L, T} t}=\sqrt{2 \bar{D}_{L, T} l / v_{D}}=D_{L, T} \sqrt{l}$ and obtain two separate equations for the longitudinal and transverse density distributions:

$$
\begin{aligned}
& \varphi_{L}(z, l)=\frac{1}{\sqrt{2 \pi l} D_{L}} \exp \left(-\frac{\left(z-z_{0}\right)^{2}}{2 D_{L}^{2} l}\right), \\
& \varphi_{T}(r, l)=\frac{1}{D_{T}^{2} l} \exp \left(-\frac{\left(r-r_{0}\right)^{2}}{2 D_{T}^{2} l}\right) .
\end{aligned}
$$

The diffusion coefficients and their dependence on the electric field strength can be obtained by calculation with MAGBOLTZ. The MAGBOLTZ data for a commonly used RPC gas mixture and for pure isobutane is shown in Fig. 2.5.

\subsubsection{Electron Multiplication}

An image of actual electron avalanches taken in a cloud chamber equipped with a parallel plate counter is shown in Fig. 2.6. Each electron starts an avalanche which grows until it hits the anode. For each electron there is a certain probability to multiply and a probability to get attached to a gas molecule. This is taken into account by introducing the Townsend coefficient $\alpha$ and the attachment coefficient $\eta$. If the avalanche contains $n$ electrons at position $z$, the probability that it will contain $n+1$ at $z+\delta z$ is given by $n \alpha \delta z$. Following the same arguments the probability that one electron gets attached (forming a negative ion) over the distance $\delta z$ is given by $n \eta \delta z$. For the average number of electrons $\bar{n}$ we therefore have the relation

$$
\frac{d \bar{n}}{d z}=(\alpha-\eta) \bar{n}
$$




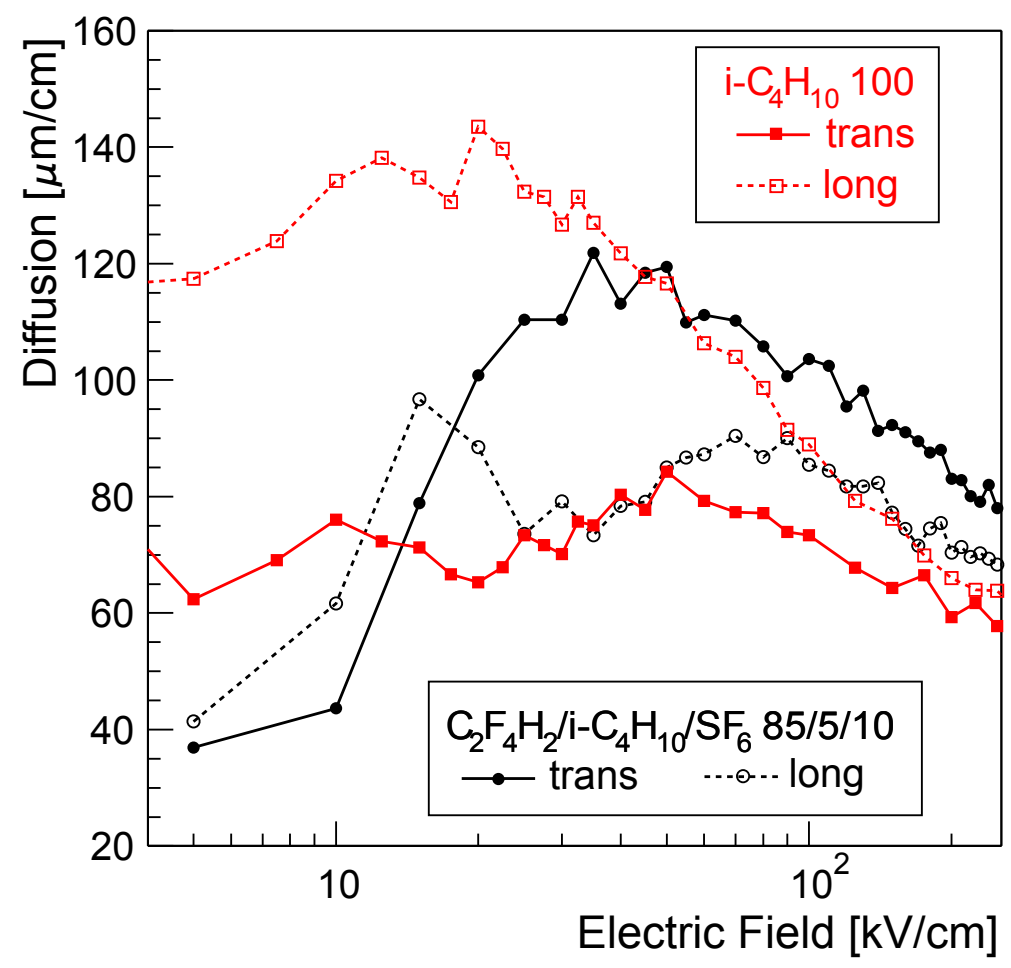

Figure 2.5: Longitudinal and transverse diffusion coefficients calculated with MAGBOLTZ for $\mathrm{C}_{2} \mathrm{~F}_{4} \mathrm{H}_{2} / \mathrm{i}-\mathrm{C}_{4} \mathrm{H}_{10} / \mathrm{SF}_{6}(85 \%, 5 \%, 10 \%)$ and pure isobutane. The data for the mixture with $0.3 \% \mathrm{SF}_{6}$ is very similar to the mixture with $10 \% \mathrm{SF}_{6}$ and is not plotted. The temperature is $T=296.15 \mathrm{~K}$ and the pressure $p=1013 \mathrm{mbar}$. Measurements are available only for much lower field strengths and do not distinguish between longitudinal and transverse diffusion [83, 84].

The solution for $\bar{n}(0)=1$ is the exponential growth law

$$
\bar{n}(z)=\exp ((\alpha-\eta) z)
$$

For non-constant Townsend and attachment coefficients $(\alpha=\alpha(z)$ and $\eta=\eta(z))$ Eq. 2.11 becomes

$$
\bar{n}(z)=\exp \left(\int_{0}^{z}(\alpha(\xi)-\eta(\xi)) d \xi\right)
$$

\subsubsection{Avalanche Statistics}

Avalanche multiplication is a stochastic process. For the statistical fluctuation different models have been suggested. Many authors use the Polya distribution which is derived 


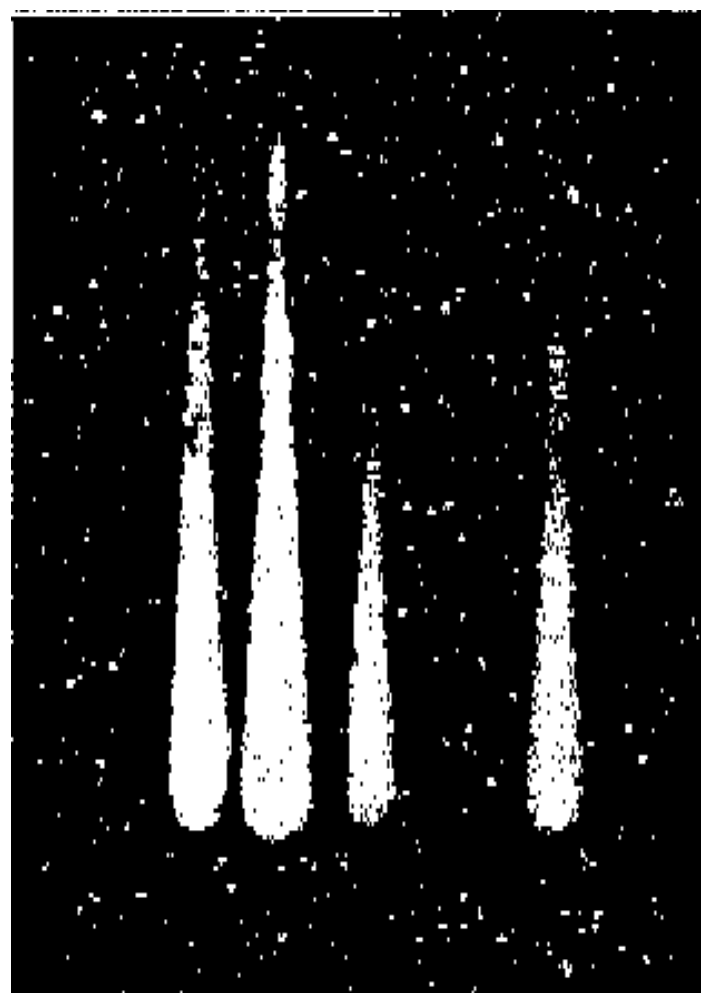

Figure 2.6: A cloud chamber photograph of electron avalanches in a parallel plate counter [38].

from the probability $p$ to find $n+1$ electrons at $z+d z$ as

$$
p=n \beta\left(b-\frac{1-b}{n}\right) d z .
$$

The parameters $\beta$ and $b$ are chosen such that the calculated results match the experimental results. Indeed the Eq. 2.13 leads to avalanche charge distributions that show a peak as do measurements [71]. The distribution Eq. 2.13 assumes that the probability to create an electron depends on the current size of the avalanche. This however misses a clear physical interpretation and describes some kind of saturation effect which we include in a different way, as we shall show later. The only justification for the use of the Polya distribution is that it parametrizes the measured curves in a nice way. In addition, this model neglects attachment.

We will instead follow a model by W. Legler [85] that describes the avalanche multiplication for electro negative gases at high fields and at large gas gain. For a detailed discussion of this model, see [1]. For the time being we assume that the Townsend and attachment coefficients $\alpha$ and $\eta$ are constant. Then the probability for an avalanche started with a single electron to have $n$ electrons at distance $z$ is 


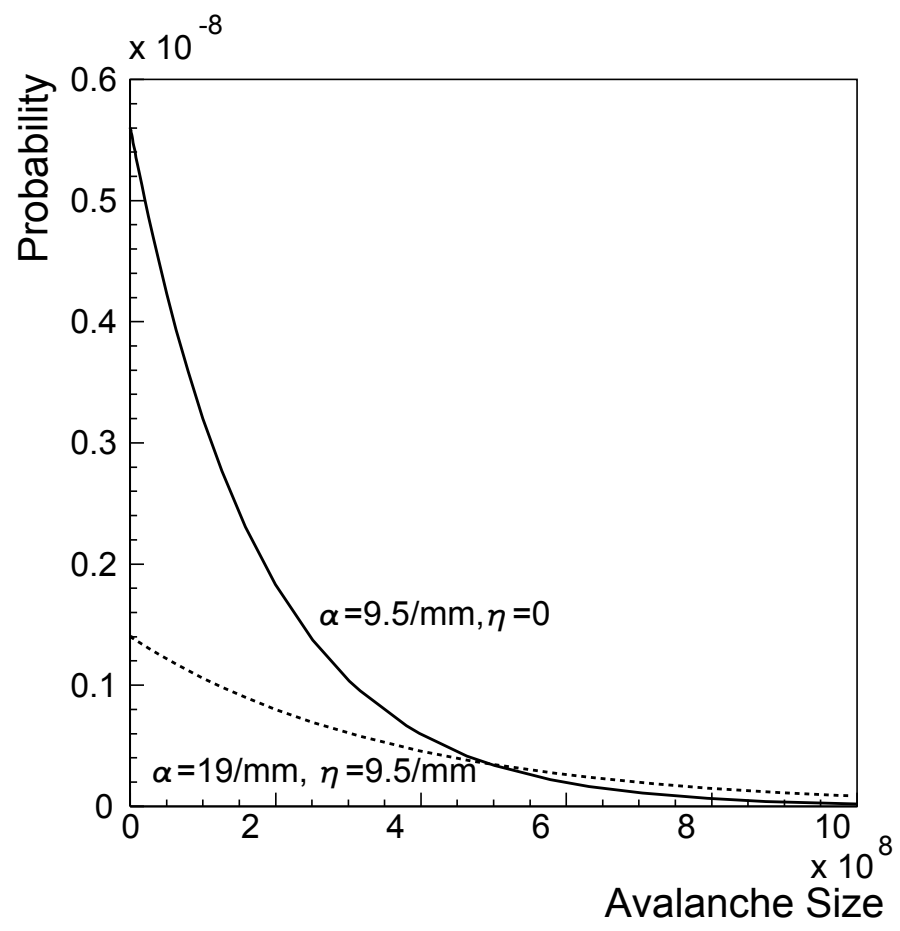

Figure 2.7: The charge distributions for avalanches starting with a single electron [1]]. The effective Townsend coefficient $\alpha-\eta$ is the same for both curves.

$$
\begin{aligned}
& P(n=0, z)=k \frac{\bar{n}(z)-1}{\bar{n}(z)-k} \\
& P(n>0, z)=\bar{n}(z)\left(\frac{1-k}{\bar{n}(z)-k}\right)^{2}\left(\frac{\bar{n}(z)-1}{\bar{n}(z)-k}\right)^{n-1} .
\end{aligned}
$$

Here we have

$$
\bar{n}(z)=e^{(\alpha-\eta) z} \quad \text { and } \quad k=\frac{\eta}{\alpha} .
$$

The variance $\sigma^{2}(z)$ of the distribution is given by

$$
\sigma^{2}(z)=\left(\frac{1+k}{1-k}\right) \bar{n}(z)(\bar{n}(z)-1)
$$

The average electron number depends on the effective Townsend coefficient $\alpha-\eta$. The variance and the distribution itself however also depend on $k=\eta / \alpha$ explicitly. 
Fig. 2.7 shows the above distribution for the same effective Townsend coefficient but different $\alpha$ and $\eta$.

In case $\alpha=\eta$ or $\alpha=0$, the distribution from Eqs. 2.14 becomes undefined and we have to use different expressions. In case $\alpha=\eta$ the probability for an avalanche started with a single electron to have $n$ electrons at distance $z$ is

$$
\begin{aligned}
& P(n=0, z)=\frac{\alpha z}{1+\alpha z} \\
& P(n>0, z)=\frac{1}{(1+\alpha z)^{2}}\left(\frac{\alpha z}{1+\alpha z}\right)^{n-1}
\end{aligned}
$$

and the variance becomes

$$
\sigma^{2}(z)=2 \alpha z
$$

In case $\alpha=0$ the probabilities are

$$
\begin{aligned}
& P(n=0, z)=1-\exp (-\eta z) \\
& P(n=1, z)=\exp (-\eta z)
\end{aligned}
$$

and the probability to find $n>1$ electrons is zero. The variance becomes

$$
\sigma^{2}(z)=\exp (-2 \eta z)(\exp (\eta z)-1)
$$

To generate a random number according to Eqs. 2.14, one draws a random uniform number $s$ from the interval $(0,1)$ and calculates

$$
\begin{array}{r}
n=0 \\
n=1+\operatorname{trunc}\left[\frac{1}{\ln \left(\frac{\bar{n}(z)-1}{\bar{n}(z)-k}\right)} \ln \left(\frac{(\bar{n}(z)-k)(s-1)}{(k-1)(\bar{n}(z))}\right)\right] \quad ; s>k \frac{\bar{n}(z)-1}{\bar{n}(z)-k} \\
\bar{n}(z)-k
\end{array}
$$

'trunc' means truncation of the decimals. In case $\bar{n}(z)$ is very large, the numerical evaluation of the logarithm in the denominator of Eq. 2.21b can become problematic and it is better to use the series expansion $\ln (1-x)=-\left(x+\frac{1}{2} x^{2}+\frac{1}{3} x^{3}+\ldots\right)$. 
To generate a random number according to Eqs. 2.17, one draws a random uniform number $s$ from the interval $(0,1)$ and calculates

$$
\begin{array}{ll}
n=0 & ; s<\frac{\alpha z}{1+\alpha z} \\
n=1+\operatorname{trunc}\left[\frac{1}{\ln \left(\frac{\alpha z}{1+\alpha z}\right)} \ln ((1-s)(1+\alpha z))\right] & ; s>\frac{\alpha z}{1+\alpha z}
\end{array}
$$

To generate a random number according to Eqs. 2.19, one draws a random uniform number $s$ from the interval $(0,1)$ and calculates

$$
\begin{array}{ll}
n=0 & ; s>\exp (-\eta z) \\
n=1 & ; s<\exp (-\eta z)
\end{array}
$$

In general $\alpha$ and $\eta$ are functions of $E / p$ where $E$ is the electric field strength sensed by the electrons and $p$ is the pressure in the gas. This functionality can be calculated with the program IMONTE [77]. For typically used gas mixtures plots are shown in Fig. 2.8. In the case of only a few charges present in the detector, the electric field $E_{0}=U_{0} / d$ between the two electrodes is uniform. The growth of the charge carriers in an avalanche is then described by Eq. 2.11. If the number of charges in the avalanche reaches large values, they influence the electric field in the gap and thus the values of $\alpha$ and $\eta$. This is the space charge effect. An approximate value of the space charge field can be deduced by assuming that the charge lies in a sphere of radius $r_{D}$. Then the field $E_{r}$ of this charged sphere at its surface is

$$
E_{r}=\frac{e_{0} n}{4 \pi \varepsilon_{0} r_{D}^{2}}
$$

where $e_{0}$ is the unit charge and $\varepsilon_{0}$ is the dielectric constant of the vacuum. With $n=10^{6}$ and $r_{D}=0.1 \mathrm{~mm}$ Eq. 2.24 gives $E_{r}=150 \mathrm{~V} / \mathrm{mm}$, which is about $3 \%$ of $E_{0}$ in Trigger RPCs and about $1.5 \%$ of $E_{0}$ in Timing RPCs. In typical RPC gas mixtures this field distortion can already produce a change of the effective Townsend coefficient of up to $10 \%$. As a consequence, we can use Eqs. 2.21, 2.22, 2.23 only locally, where we assume that $\alpha$ and $\eta$ are constant. 

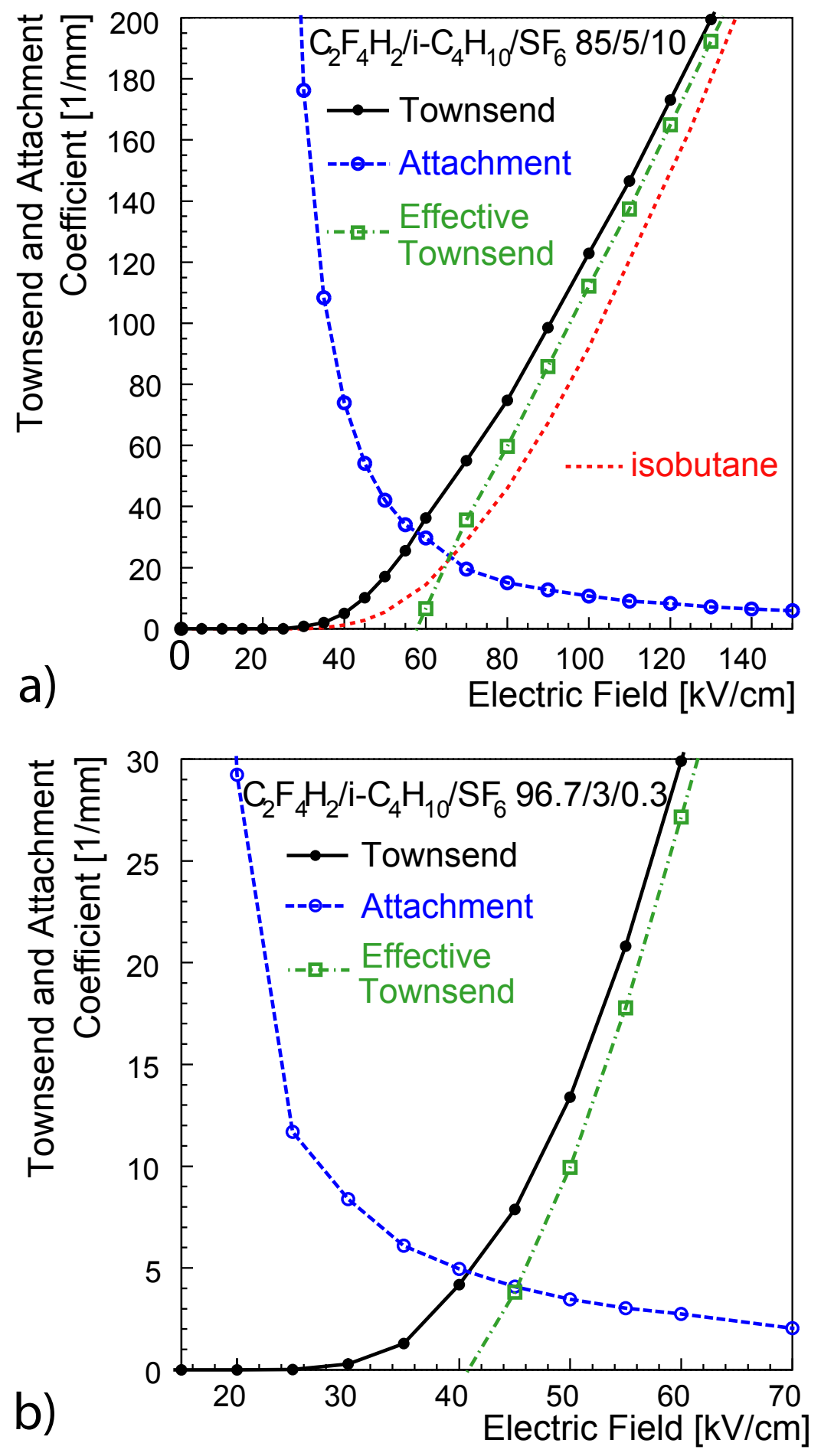

Figure 2.8: Townsend and attachment coefficients for different gas mixtures calculated with IMONTE [77]. a) for the Timing RPC gas mixture and for pure isobutane, b) for the Trigger RPC gas mixture. The temperature of the gas is $T=296.15 \mathrm{~K}$ and the pressure $p=1013$ mbar. 


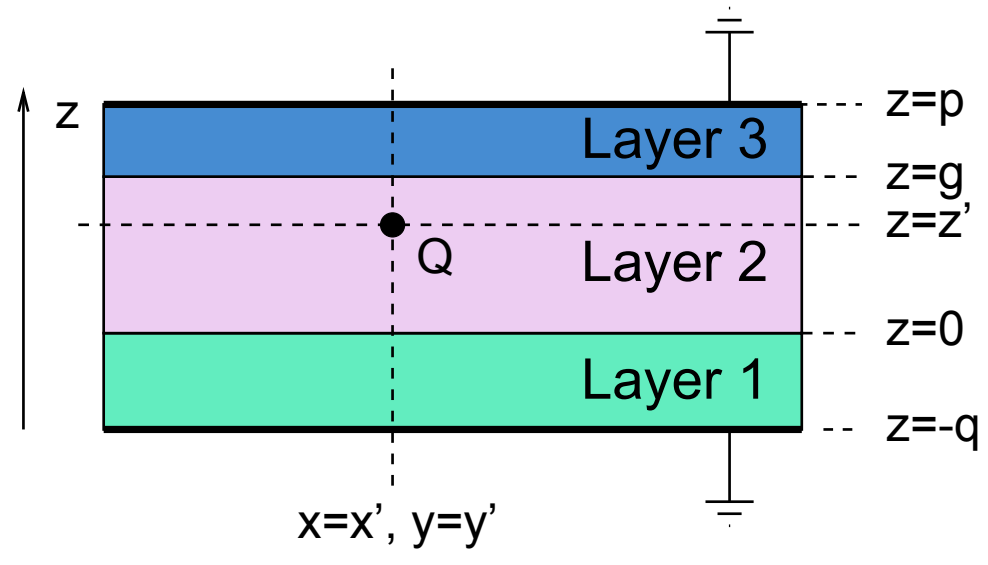

Figure 2.9: The three layer geometry similar to that of resistive plate chambers. The layers have different dielectric constants and different thicknesses. There is a point charge in layer 2 at $\left(x=x^{\prime}, y=y^{\prime}, z=z^{\prime}\right)$.

\subsection{Electrostatics of Three Layer Geometries}

In this section we present an analytic solution for the potential of a point charge in a three layer geometry like an RPCs [4]. It is an essential indegredient if we want to calculate the electric field that is sensed by an electron in an avalanche at large gain in the gas gap of an RPC. The RPC is treated as an infinite plane condenser comprising three homogeneous isolating parallel dielectric layers. A detailed discussion of the derivation of the solutions can be found in [3]. There also the solution for the potential of a point charge in an infinite plane condenser with one homogeneous isolating dielectric layer is presented. It can be used for calculations of space charge fields in parallel plate chambers with metallic electrodes, like the Parallel Plate Avalanche Chamber (PPAC).

The resistive plates of RPCs have volume resistivities of about $10^{9}$ to $10^{12} \Omega \mathrm{cm}$. In section 1.3 .4 we mentioned that this resistivity results in relaxation times $\tau$ that are needed for the charges entering the resistive layers to decompose. We found values for $\tau$ between $10 \mathrm{~ms}$ for the bakelite resistive layer and up to $1 \mathrm{~s}$ for a glass resistive layer. The timescale of an avalanche on the other hand, if we only consider the electrons, is a few nanoseconds, which is a difference of six orders of magnitude. For such fast processes, the resistive electrode material can be treated as an insulator.

\subsubsection{Potential of a Point Charge for the Three Layer Problem}

Fig. 2.9 shows the geometry investigated in this section. The point charge is at position $\left(x^{\prime}, y^{\prime}, z^{\prime}\right)$. Layer $2-$ in the case of an RPC the gas gap - is at $0 \leq z \leq g$. It has a dielectric constant $\varepsilon_{2}$, while layers 1 and 3 have dielectric constants $\varepsilon_{1}$ and $\varepsilon_{3}$ 
respectively. The $\varepsilon_{i}$ represent the full dielectric constants, i.e. they are $\varepsilon_{0}$ times the relative permittivity of the medium, where $\varepsilon_{0}$ is the dielectric constant of the vacuum. We assume that the $\varepsilon_{i}$ are constant. We will use cylindrical coordinates and write the distance between the point charge (at $\vec{r}^{\prime}$ ) and the point of observation (at $\vec{r}$ ) as

$$
\begin{aligned}
& R^{2}=\left|\vec{r}-\vec{r}^{\prime}\right|^{2}=\left(x-x^{\prime}\right)^{2}+\left(y-y^{\prime}\right)^{2}+\left(z-z^{\prime}\right)^{2} \\
& =r^{2}-2 r r^{\prime} \cos \left(\phi-\phi^{\prime}\right)+r^{\prime 2}+\left(z-z^{\prime}\right)^{2} \\
& =\quad P^{2}+\left(z-z^{\prime}\right)^{2} \text {. }
\end{aligned}
$$

An integral representation of the potential in layer $2(0<z<g)$ for a point charge sitting in layer 2 is given by [4]

$$
\begin{gathered}
\Phi\left(r, \phi, z, r^{\prime}, \phi^{\prime}, z^{\prime}\right)=\frac{Q}{4 \pi \varepsilon_{2}}\left[\frac{1}{\sqrt{P^{2}+\left(z-z^{\prime}\right)^{2}}}-\frac{\left(\varepsilon_{1}-\varepsilon_{2}\right)}{\left(\varepsilon_{1}+\varepsilon_{2}\right) \sqrt{P^{2}+\left(z+z^{\prime}\right)^{2}}}\right. \\
-\frac{\left(\varepsilon_{3}-\varepsilon_{2}\right)}{\left(\varepsilon_{3}+\varepsilon_{2}\right) \sqrt{P^{2}+\left(2 g-z-z^{\prime}\right)^{2}}} \\
\left.+\quad \frac{1}{\left(\varepsilon_{1}+\varepsilon_{2}\right)\left(\varepsilon_{2}+\varepsilon_{3}\right)} \int_{0}^{\infty} d \kappa J_{0}(\kappa P) \frac{R\left(\kappa, z, z^{\prime}\right)}{D(\kappa)}\right], \\
0 \leq z \leq g ;
\end{gathered}
$$

where $J_{0}$ is the Bessel function of the first kind and of order zero. The denominator $D(\kappa)$ in the integral is given by

$$
\begin{aligned}
D(\kappa)= & \left(\varepsilon_{1}+\varepsilon_{2}\right)\left(\varepsilon_{2}+\varepsilon_{3}\right)\left(1-e^{-2 \kappa(p+q)}\right) \\
& -\left(\varepsilon_{1}-\varepsilon_{2}\right)\left(\varepsilon_{2}+\varepsilon_{3}\right)\left(e^{-2 \kappa p}-e^{-2 \kappa q}\right) \\
& -\left(\varepsilon_{1}+\varepsilon_{2}\right)\left(\varepsilon_{2}-\varepsilon_{3}\right)\left(e^{-2 \kappa(p-g)}-e^{-2 \kappa(q+g)}\right) \\
& +\left(\varepsilon_{1}-\varepsilon_{2}\right)\left(\varepsilon_{2}-\varepsilon_{3}\right)\left(e^{-2 \kappa g}-e^{-2 \kappa(p+q-g)}\right)
\end{aligned}
$$

and the numerator is 


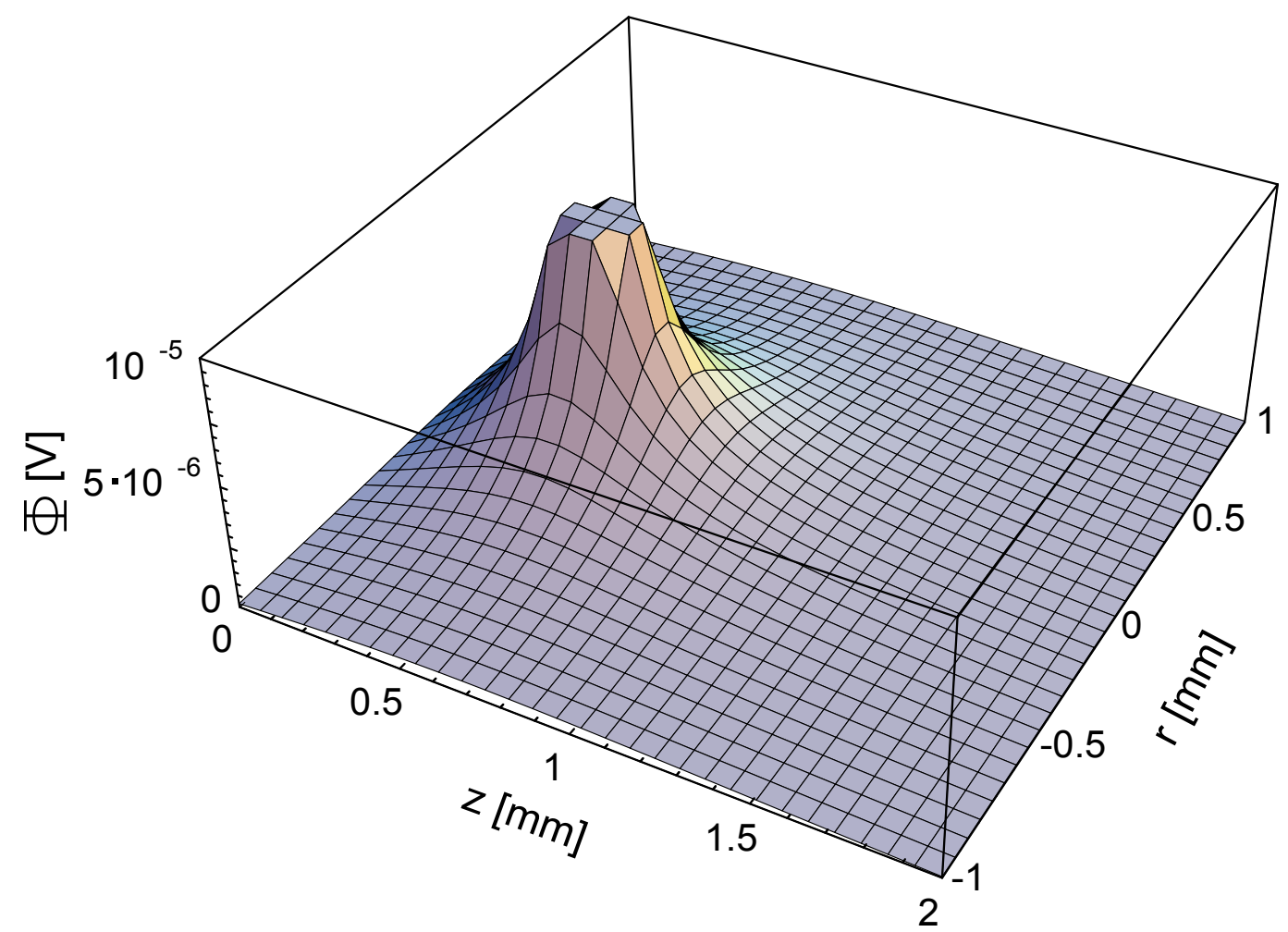

Figure 2.10: The potential of a point charge (Eq. 2.26) at position $z=0.5, r=0$ in the plane $\phi=\phi^{\prime}=0$ in a $2 \mathrm{~mm}$ gap RPC. We used $\varepsilon_{1}=\varepsilon_{3}=10 \varepsilon_{0}, \varepsilon_{2}=\varepsilon_{0}, P=0$, $g=q=2$ and $p=4$.

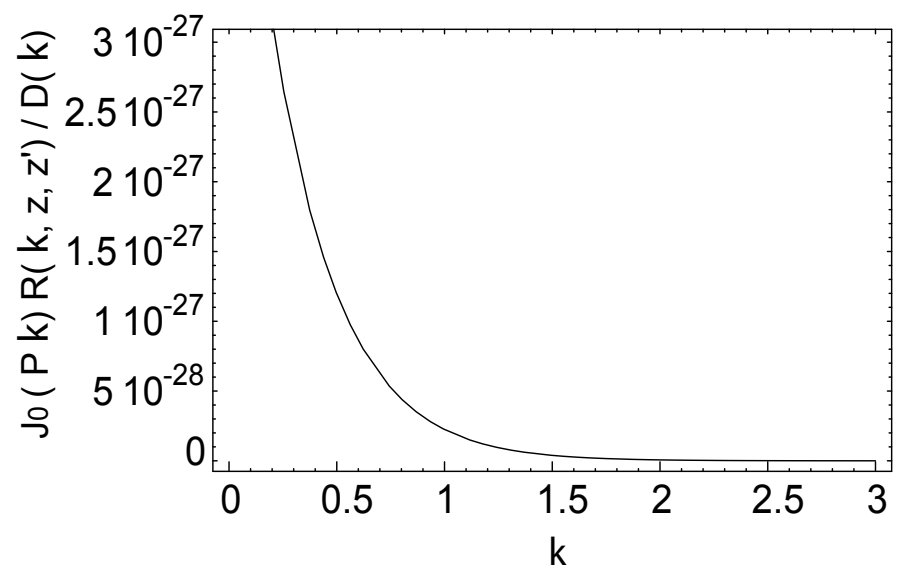

Figure 2.11: A plot of the integrand of Eq. 2.26 for $\varepsilon_{1}=\varepsilon_{3}=10 \varepsilon_{0}, \varepsilon_{2}=\varepsilon_{0}, P=0$, $z=1, z^{\prime}=0.48, g=q=2$ and $p=4$. 

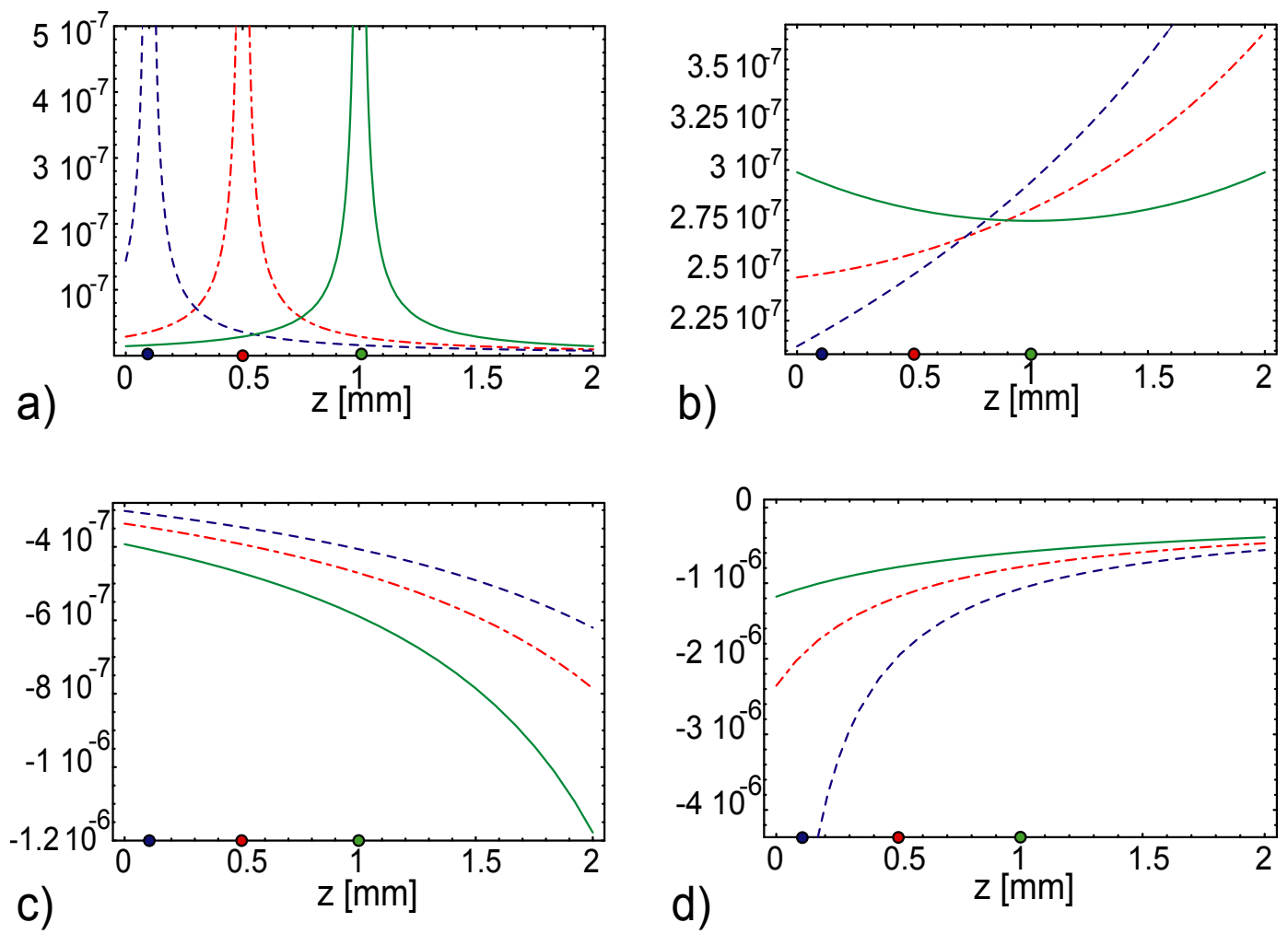

Figure 2.12: The four different terms of Eq. 2.26 are plotted for three different positions of the charges: $z^{\prime}=0.1 \mathrm{~mm}, 0.5 \mathrm{~mm}, 1 \mathrm{~mm}$. The positions are indicated by the circles. a) The first term, which is the potential of a free charge. b) The integral term. c) The second term, which is the potential of a mirror charge at $\left({ }^{\prime} r, \phi^{\prime}, 2 g-z^{\prime}\right)$. d) The third term, which is the potential of a mirror charge at $\left(r^{\prime}, \phi^{\prime},-z^{\prime}\right)$.

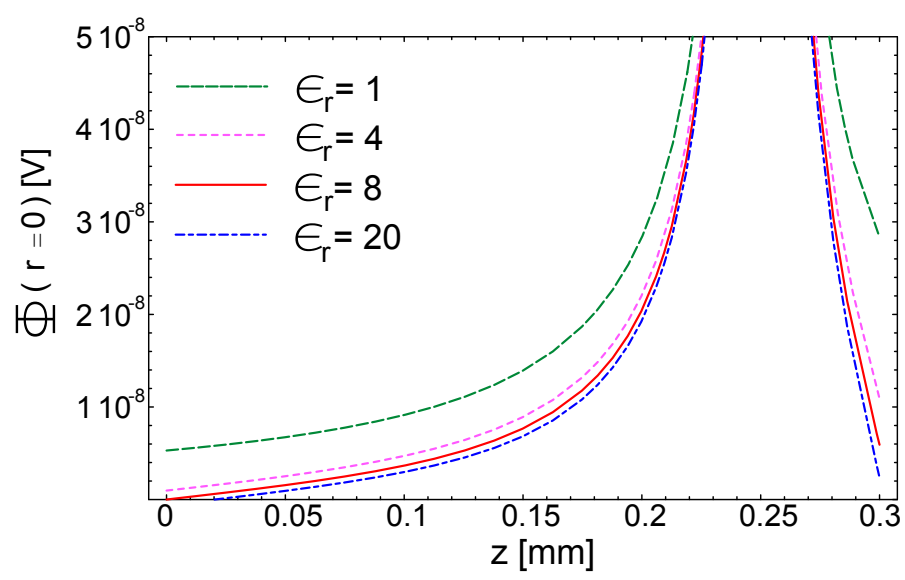

Figure 2.13: A plot of the potential of a point charge (Eq. 2.26) in a $0.3 \mathrm{~mm}$ gap RPC for different values of $\varepsilon_{r}$ with $\varepsilon_{1}=\varepsilon_{3}=\varepsilon_{r} \varepsilon_{0}$ and $\varepsilon_{2}=\varepsilon_{0}$. 


$$
\begin{aligned}
& R\left(\kappa ; z, z^{\prime}\right)= \\
& \left(\varepsilon_{1}+\varepsilon_{2}\right)^{2}\left(\varepsilon_{2}+\varepsilon_{3}\right)^{2}\left[e^{\kappa\left(-2 p-2 q+z-z^{\prime}\right)}+e^{\kappa\left(-2 p-2 q-z+z^{\prime}\right)}\right]- \\
& \left(\varepsilon_{1}+\varepsilon_{2}\right)^{2}\left(\varepsilon_{2}-\varepsilon_{3}\right)^{2} e^{\kappa\left(-4 g-2 q+z+z^{\prime}\right)}- \\
& 4 \varepsilon_{1} \varepsilon_{2}\left(\varepsilon_{2}+\varepsilon_{3}\right)^{2} e^{\kappa\left(-2 q-z-z^{\prime}\right)}-\left(\varepsilon_{1}-\varepsilon_{2}\right)^{2}\left(\varepsilon_{2}+\varepsilon_{3}\right)^{2} e^{\kappa\left(-2 p-z-z^{\prime}\right)}- \\
& \left(\varepsilon_{1}^{2}-\varepsilon_{2}^{2}\right)\left(\varepsilon_{2}-\varepsilon_{3}\right)^{2} e^{\kappa\left(-4 g+z+z^{\prime}\right)}+ \\
& \left(\varepsilon_{1}^{2}-\varepsilon_{2}^{2}\right)\left(\varepsilon_{2}+\varepsilon_{3}\right)^{2}\left[-e^{\kappa\left(-2 p-2 q-z-z^{\prime}\right)}+e^{\kappa\left(-2 p+z-z^{\prime}\right)}+e^{\kappa\left(-2 p-z+z^{\prime}\right)}\right]- \\
& 4\left(\varepsilon_{1}^{2}-\varepsilon_{2}^{2}\right) \varepsilon_{2} \varepsilon_{3} e^{\kappa\left(-2 p-2 q+z+z^{\prime}\right)}-4\left(\varepsilon_{1}+\varepsilon_{2}\right)^{2} \varepsilon_{2} \varepsilon_{3} e^{\kappa\left(-2 p+z+z^{\prime}\right)}+ \\
& \left(\varepsilon_{1}-\varepsilon_{2}\right)^{2}\left(\varepsilon_{2}{ }^{2}-\varepsilon_{3}{ }^{2}\right) e^{\kappa\left(-2 g-z-z^{\prime}\right)}+4 \varepsilon_{1} \varepsilon_{2}\left(\varepsilon_{2}^{2}-\varepsilon_{3}^{2}\right) e^{\kappa\left(2 g-2 p-2 q-z-z^{\prime}\right)}+ \\
& \left(\varepsilon_{1}+\varepsilon_{2}\right)^{2}\left(\varepsilon_{2}{ }^{2}-\varepsilon_{3}{ }^{2}\right) \\
& {\left[-e^{\kappa\left(-2 g-2 q+z-z^{\prime}\right)}-e^{\kappa\left(-2 g-2 q-z+z^{\prime}\right)}+e^{\kappa\left(-2 g-2 p-2 q+z+z^{\prime}\right)}\right]+} \\
& \left(\varepsilon_{1}^{2}-\varepsilon_{2}^{2}\right)\left(\varepsilon_{2}^{2}-\varepsilon_{3}^{2}\right) \\
& {\left[e^{\kappa\left(-2 g-2 q-z-z^{\prime}\right)}-e^{\kappa\left(-2 g+z-z^{\prime}\right)}-e^{\kappa\left(-2 g-z+z^{\prime}\right)}+e^{\kappa\left(-2 g-2 p+z+z^{\prime}\right)}\right] .}
\end{aligned}
$$

Eq. 2.26 consists of four terms. A plot of all four terms can be found in Fig. 2.12. The first term is the potential of a free point charge at $\left(r^{\prime}, \phi^{\prime}, z^{\prime}\right)$, while the second and third terms are the potentials of two mirror charges situated at $\left(r^{\prime}, \phi^{\prime}, 2 g-z^{\prime}\right)$ and $\left(r^{\prime}, \phi^{\prime},-z^{\prime}\right)$. The fourth term is a correction term. The integral behaves very nicely in terms of fast convergence (see Fig. 2.11). From Fig. 2.12 it is also obvious that the two terms that belong to the potential of a free point charge and the mirror charge that is closer to the point of observation dominate the result. The influence of the relative permittivity of layers 1 and 3 (in the case of the RPC the two resistive layers) on the potential is shown in Fig. 2.13. We see that only for $\varepsilon_{r} \rightarrow 1$ the influence is large.

Eq. 2.26 is only applicable for calculating the potential in the central layer, if the point charge is also situated in the central layer. In [3] all 9 different analytic solutions for the potential in layers 1, 2 or 3 for a point charge sitting in layers 1, 2 or 3 are given.

\subsubsection{Electric Field of a Point Charge for the Three Layer Problem}

The expressions for the electric fields are found from the potential (Eq. 2.26) by derivation. 


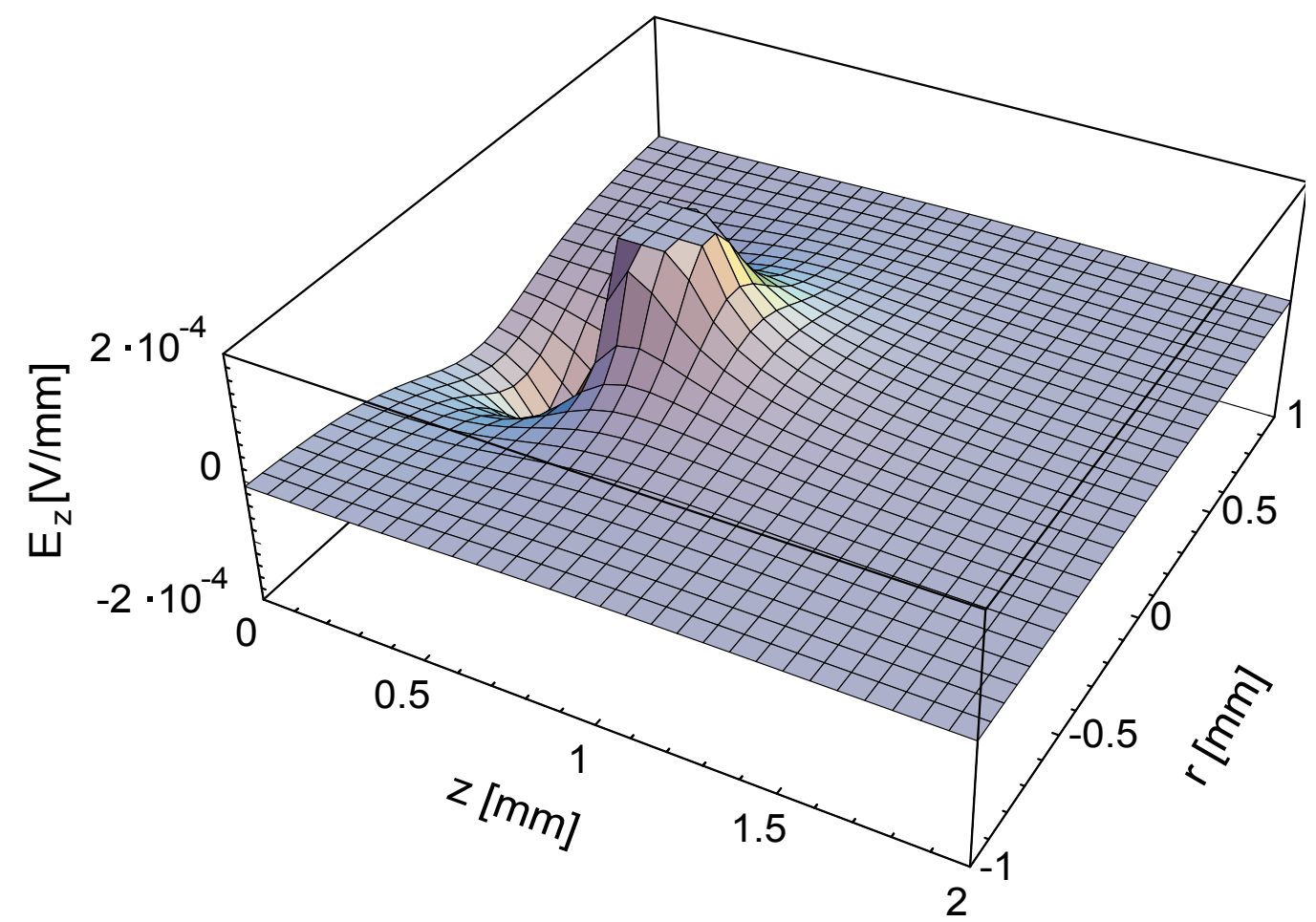

Figure 2.14: The $z$-component of the electric field of a point charge at position $z=0.5$, $r=0$ in the plane $\phi=\phi^{\prime}=0$ in a $2 \mathrm{~mm}$ gap RPC.

$$
\begin{aligned}
& E_{r}\left(r, \phi, z, r^{\prime}, \phi^{\prime}, z^{\prime}\right)=-\frac{\partial \Phi}{\partial r}\left(r, \phi, z, r^{\prime}, \phi^{\prime}, z^{\prime}\right), \\
& E_{\phi}\left(r, \phi, z, r^{\prime}, \phi^{\prime}, z^{\prime}\right)=-\frac{1}{r} \frac{\partial \Phi}{\partial \phi}\left(r, \phi, z, r^{\prime}, \phi^{\prime}, z^{\prime}\right), \\
& E_{z}\left(r, \phi, z, r^{\prime}, \phi^{\prime}, z^{\prime}\right)=-\frac{\partial \Phi}{\partial z}\left(r, \phi, z, r^{\prime}, \phi^{\prime}, z^{\prime}\right) .
\end{aligned}
$$

Fig. 2.14 shows the $z$-component of the electric field of a point charge in an RPC, following Eq. 2.29c. A comparison of the electric field of a point charge in the gas gap of an RPC to the electric field of a free point charge is shown in Fig. 2.15. We find that especially close to the resistive layers the field differs by up to $80 \%$ from the field of the free point charge. Fig. 2.12 shows that close to the resistive layers the respective close mirror charge becomes important while the far mirror charge term and the integral term have only a small influence. This is shown more clearly in Fig. 2.16 where the full solution for the $z$-components of the electric field following Eq. (2.29c) is compared to the field of a free point charge and of one mirror charge in the near resistive layer. For fast computations Eq. 2.26 can be approximated by omitting the second and third terms. In that case the three components of the electric field are 


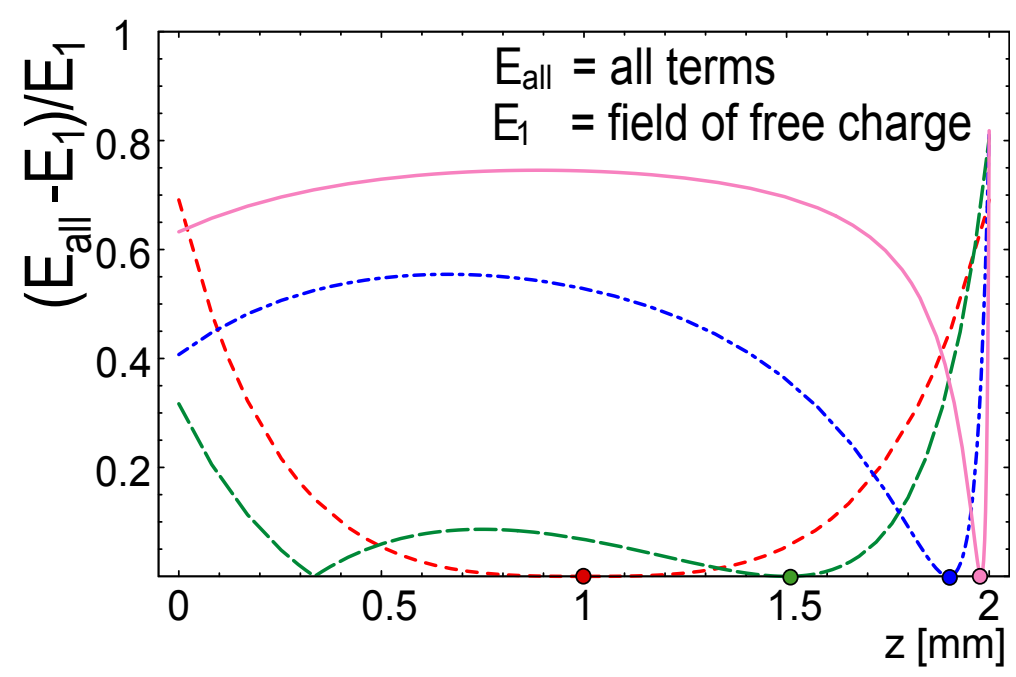

Figure 2.15: A comparison of the solutions for the $z$-components of the electric field $E_{1}$ of a free point charge. The deviation is plotted for $\varepsilon_{1}=\varepsilon_{3}=10 \varepsilon_{0}, \varepsilon_{2}=\varepsilon_{0}, P=0$, $g=q=2$ and $p=4$ and for four different positions of the charge in a $2 \mathrm{~mm}$ gap. The positions of the charges are indicated by the dots: $z=1,1.5,1.9,1.98 \mathrm{~mm}$. Close to the resistive layers, especially if the charge itself is situated close to the resistive layers, the solutions deviate by up to $80 \%$.

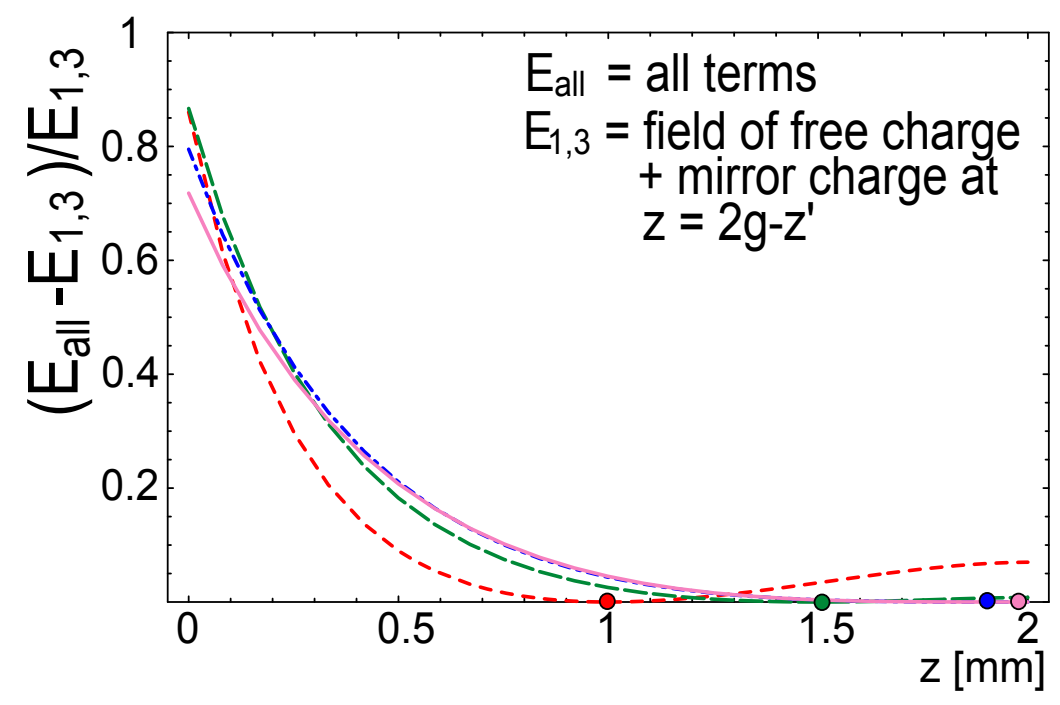

Figure 2.16: A comparison of the solutions for the $z$-components of the electric field $E_{\text {all }}\left(\right.$ Eq. (2.29c)) to the field of a free point charge $E_{1}$ and the field $E_{3}$ of a mirror charge at $z=2 g-z^{\prime}$. The deviation is plotted for $\varepsilon_{1}=\varepsilon_{3}=10 \varepsilon_{0}, \varepsilon_{2}=\varepsilon_{0}, P=0$, $g=q=2$ and $p=4$ and for four different positions of the charge in a $2 \mathrm{~mm}$ gap. The positions of the charges are indicated by the dots: $z=1,1.5,1.9,1.98 \mathrm{~mm}$. 


$$
\begin{aligned}
E_{r}\left(r, \phi, z, r^{\prime}, \phi^{\prime}, z^{\prime}\right) \approx & \frac{Q}{4 \pi \varepsilon_{2}}\left[\frac{r-r^{\prime} \cos \left(\phi-\phi^{\prime}\right)}{\left(P^{2}+\left(z-z^{\prime}\right)^{2}\right)^{\frac{3}{2}}}\right. \\
+ & \left.\frac{\left(\varepsilon_{2}-\varepsilon_{3}\right)}{\left(\varepsilon_{2}+\varepsilon_{3}\right)} \frac{r-r^{\prime} \cos \left(\phi-\phi^{\prime}\right)}{\left(P^{2}+\left(2 g-z-z^{\prime}\right)^{2}\right)^{\frac{3}{2}}}\right], \\
E_{\phi}\left(r, \phi, z, r^{\prime}, \phi^{\prime}, z^{\prime}\right) \approx & \quad \frac{Q}{4 \pi \varepsilon_{2}}\left[\frac{r^{\prime} \sin \left(\phi-\phi^{\prime}\right)}{\left(P^{2}+\left(z-z^{\prime}\right)^{2}\right)^{\frac{3}{2}}}\right. \\
+ & \left.\frac{\left(\varepsilon_{2}-\varepsilon_{3}\right)}{\left(\varepsilon_{2}+\varepsilon_{3}\right)} \frac{r^{\prime} \sin \left(\phi-\phi^{\prime}\right)}{\left(P^{2}+\left(2 g-z-z^{\prime}\right)^{2}\right)^{\frac{3}{2}}}\right], \\
E_{z}\left(r, \phi, z, r^{\prime}, \phi^{\prime}, z^{\prime}\right) \approx & \frac{Q}{4 \pi \varepsilon_{2}}\left[\frac{z-z^{\prime}}{\left(P^{2}+\left(z-z^{\prime}\right)^{2}\right)^{\frac{3}{2}}}\right. \\
- & \left.\frac{\left(\varepsilon_{2}-\varepsilon_{3}\right)}{\left(\varepsilon_{2}+\varepsilon_{3}\right)} \frac{2 g-z-z^{\prime}}{\left(P^{2}+\left(2 g-z-z^{\prime}\right)^{2}\right)^{\frac{3}{2}}}\right] .
\end{aligned}
$$

\subsection{Signal Induction Process}

The movement of the charges in the detector induces a current signal on the read out electrodes. Because of their small drift velocity, the current signal induced by the drifting ions is much smaller than the current induced by the electrons. The induced current signal of $N(t)$ charge carriers in a cluster that is moving with the velocity $\vec{v}_{D}(t)=\dot{\vec{x}}(t)$ at time $t$ is given by [86, 87]

$$
i(t)=\vec{E}_{w}(\vec{x}(t)) \cdot \vec{v}_{D}(t) e_{0} N(t),
$$

where $e_{0}$ is the unit charge and $\vec{E}_{w}$ is the electric field in the gas gap if we put one RPC read out strip on $1 \mathrm{~V}$ and ground all other electrodes. The value $\vec{E}_{w}$ is called the weighting field. It should not be confused with the actual electric field. A schematic plot of the weighting field and the signal induction process is given in Fig. 2.17. For $n_{C l}$ clusters moving in the gas gap of an RPC, the induced current signal is the sum over all clusters

$$
i(t)=\sum_{j=1}^{n_{C l}} \vec{E}_{w}\left(\vec{x}_{j}(t)\right) \cdot \vec{v}_{D j}(t) e_{0} N_{j}(t)
$$




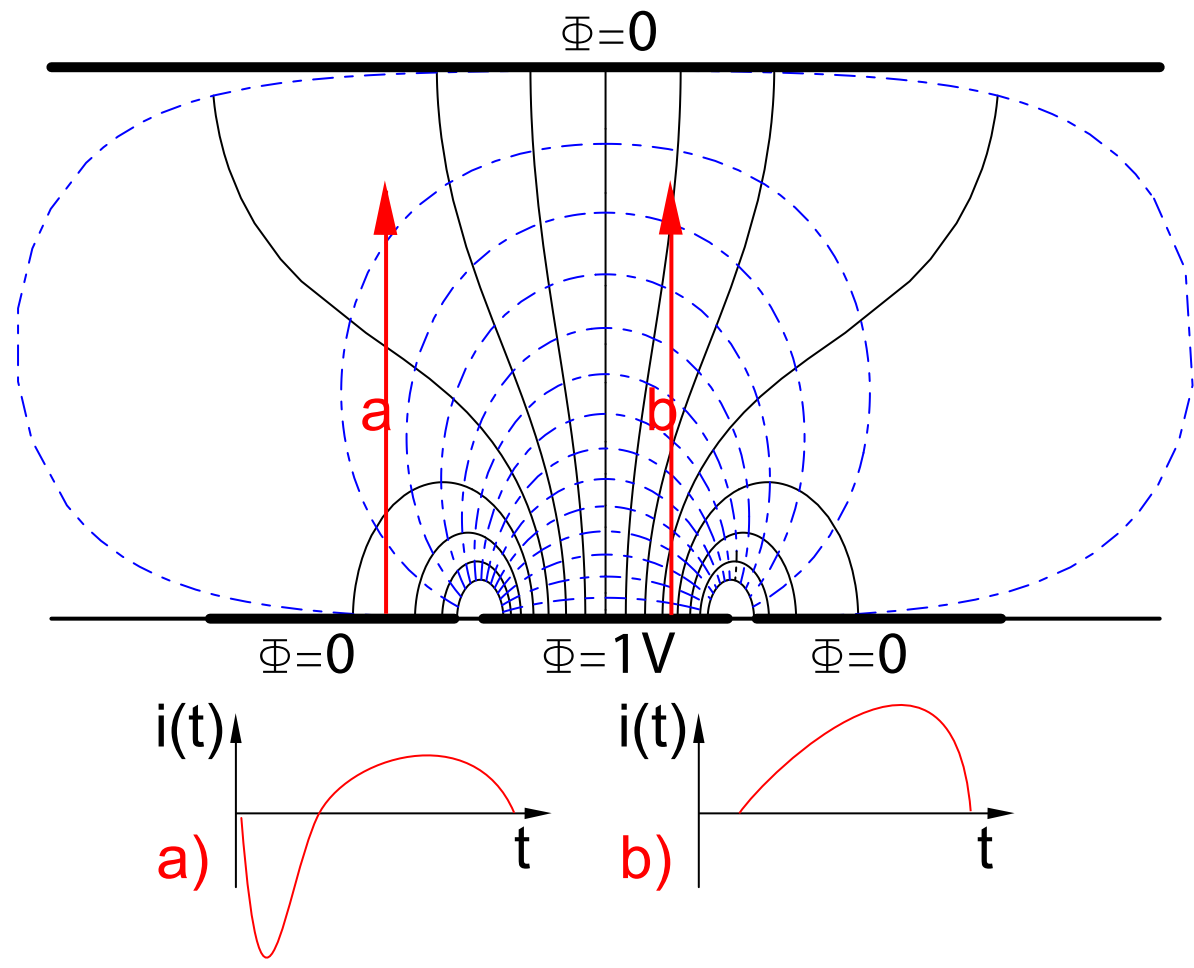

Figure 2.17: A schematic plot of the weighting field in a strip detector and the signal induction process in two examples. The induced current is calculated using the scalar product of the weighting field vector and the velocity vector(s) of the moving charge(s).

\subsubsection{Weighting Field in the Gas Gap of an RPC}

In this section we give analytic formulas for the weighting field of a strip electrode in the three layer geometry shown in Fig. 2.18. The read out strip has width $w$ and infinite length.

We want to calculate the induced signal on a certain read out strip. Then the weighting potential $\Phi_{1}(x, z)$ is the potential in the central layer of the described geometry, if this strip is put on $1 \mathrm{~V}$ and all other electrodes are grounded. It is given by [4]

$$
\Phi_{1}(x, z)=\varepsilon_{1} \frac{2}{\pi} \int_{0}^{\infty} d \kappa \cos (\kappa x) \sin \left(\kappa \frac{w}{2}\right) \frac{1}{\kappa} F_{1}(\kappa, z)
$$

With this we calculate the components of the weighting field vertical to the resistive layers $\left(E_{z}\right)$ and parallel to the resistive layers $\left(E_{x}\right)$. 


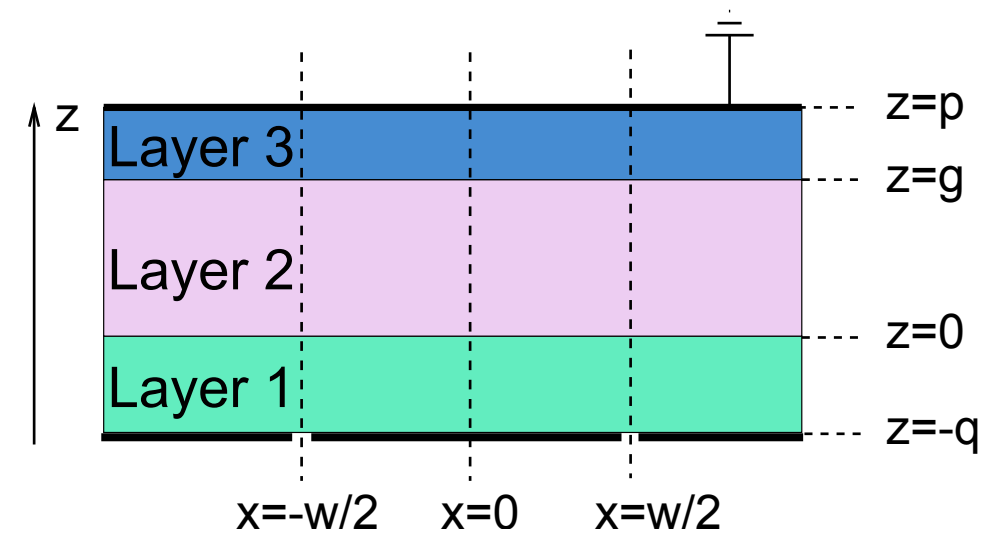

Figure 2.18: The three layer geometry investigated. The width of the readout strip is $w$.

$$
\begin{aligned}
& E_{x}(x, z)=-\varepsilon_{1} \frac{2}{\pi} \int_{0}^{\infty} d \kappa \sin (\kappa x) \sin \left(\kappa \frac{w}{2}\right) F_{1}(\kappa, z) \\
& E_{z}(x, z)=\varepsilon_{1} \frac{2}{\pi} \int_{0}^{\infty} d \kappa \cos (\kappa x) \sin \left(\kappa \frac{w}{2}\right) F_{2}(\kappa, z)
\end{aligned}
$$

with

$$
\begin{aligned}
F_{1}(\kappa, z)= & \frac{2}{D(\kappa)}\left[\left(\varepsilon_{2}+\varepsilon_{3}\right)\left(e^{-\kappa(q+z)}-e^{-\kappa(2 p+q-z)}\right)\right. \\
& \left.+\left(\varepsilon_{2}-\varepsilon_{3}\right)\left(e^{-\kappa(2 g+q-z)}-e^{-\kappa(2 p+q-2 g+z)}\right)\right]
\end{aligned}
$$

and

$$
\begin{aligned}
F_{2}(\kappa, z)= & -\frac{2}{D(\kappa)}\left[\left(\varepsilon_{2}+\varepsilon_{3}\right)\left(e^{-\kappa(q+z)}+e^{-\kappa(2 p+q-z)}\right)\right. \\
& \left.-\left(\varepsilon_{2}-\varepsilon_{3}\right)\left(e^{-\kappa(q+2 g-z)}+e^{-\kappa(2 p+q-2 g+z)}\right)\right] .
\end{aligned}
$$

$D(\kappa)$ was defined in Eq. 2.27. The two components of the weighting field are plotted for a $0.3 \mathrm{~mm}$ gap and a $32 \mathrm{~mm}$ read out strip in Fig 2.19. Since the electrons in the gas gap of an RPC generally move parallel to the $z$-axis and since Eq. 2.33 contains the scalar product of the velocity and the weighting field vector, the component $E_{z}(x, z)$ is the important one for the calculation of the induced current. Moreover, $E_{x}(x, z)$ is 

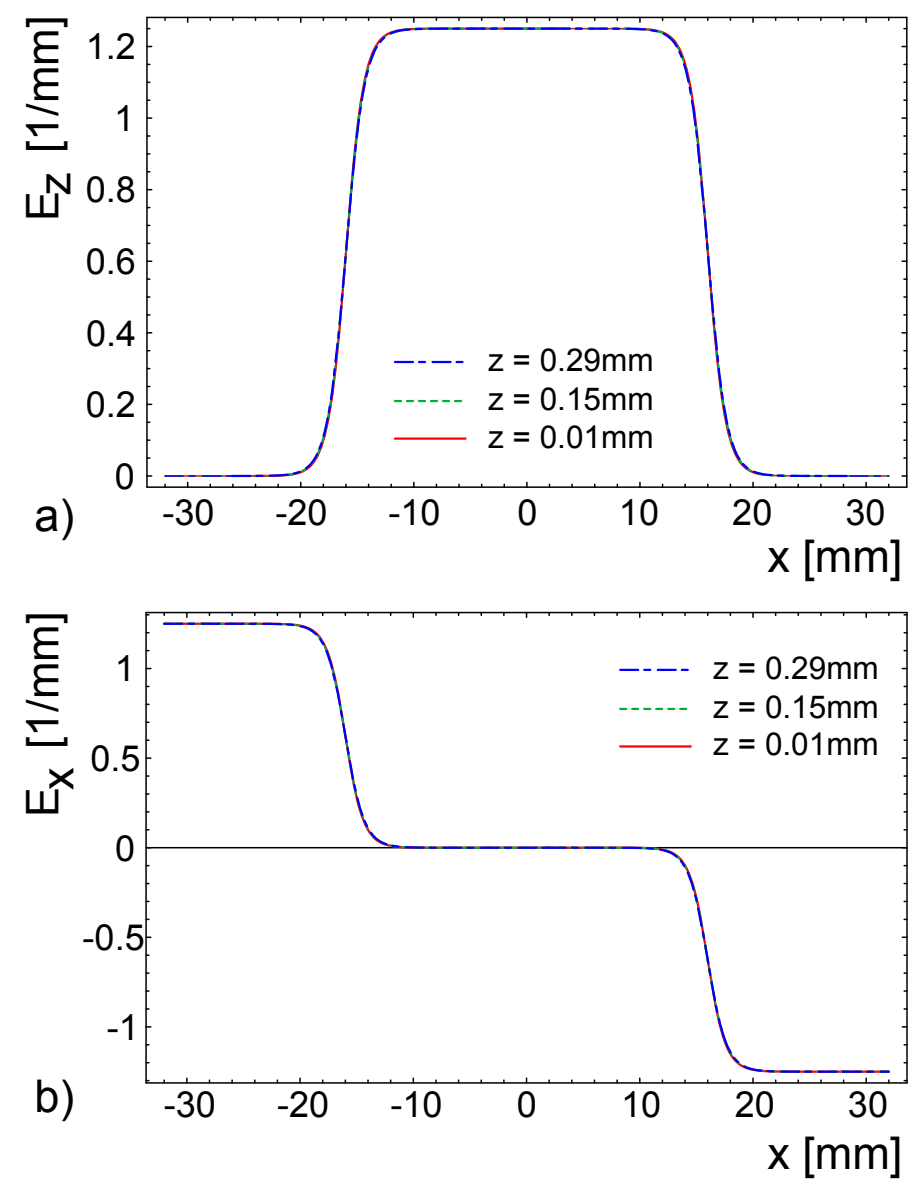

Figure 2.19: A plot of the $z$-component (a) and the $x$-component (b) of the weighting field following Eqs 2.36 for three different positions in the gap. The gap size is $g=$ $0.3 \mathrm{~mm}$, the strip width is $w=32 \mathrm{~mm}$. $\varepsilon_{1}=\varepsilon_{3}=8 \varepsilon_{0}, \varepsilon_{2}=\varepsilon_{0}, q=2 \mathrm{~mm}, p=g+q$. The plots for the three $z$-positions are almost indistinguishable.

zero over the largest area of the strip. In Fig. 2.19 we find that the values of $E_{z}(x, z)$ are approximately equal for different $z$-positions in the gap. In the limit of a very wide strip the field Eq. (2.36b) in the center of the strip $(x=0)$ approaches

$$
E_{z}=\frac{\varepsilon_{1} \varepsilon_{3}}{\varepsilon_{2} \varepsilon_{3} q+\varepsilon_{1} \varepsilon_{2} p+\left(\varepsilon_{1} \varepsilon_{3}-\varepsilon_{1} \varepsilon_{2}\right) g} .
$$

independent of $z$. For the typical single gap RPC geometry with two resistive electrodes of thickness $q=p-g$ and dielectric constants $\varepsilon_{1}=\varepsilon_{3}=\varepsilon_{0} \varepsilon_{r}$ (for example, see Fig. (1.11) Eq. 2.39 becomes 


$$
E_{z}=\frac{\varepsilon_{r}}{2 q+g \varepsilon_{r}}
$$

Here $\varepsilon_{r}$ is the relative permittivity of the resistive layers. It does not include $\varepsilon_{0}$ ! We find typical values for the weighting fields for single gap Timing RPCs of $E_{z}=$ $1.25 / \mathrm{mm}$. For a single gap Trigger RPC with $q=g=2 \mathrm{~mm}$ we find $E_{z}=0.417 / \mathrm{mm}$.

\subsubsection{Induced Charge}

The induced charge $Q_{\text {ind }}$ can be calculated as the integral of Eq. 2.34:

$$
Q_{i n d}=\int_{0}^{T} d t \sum_{j=1}^{n_{c l}} \vec{E}_{z}\left(\vec{x}_{j}(t)\right) \cdot \vec{v}_{j}(t) e N_{j}(t)
$$

where $T$ is the total signal time.

\subsection{Streamers}

In this section we summarize briefly the phenomenon of streamers. An avalanche can transform into a streamer at a high gas gain when photons start to contribute to its propagation [38]. The propagation velocity of streamers was measured to be significantly higher than the drift velocity of the normal avalanche [38]. At a later stage the streamer can further evolve into a glow discharge, a filamentary discharge and a spark [88]. However, the later discharge stages require a considerable current to flow in the gap, which is suppressed by the high resistivity of the RPC electrodes.

[38] and optical methods [89, 90] suggest that there are two different generation mechanisms for streamers:

1. A relatively slow mechanism, which needs a number of consecutive avalanches to take place in the gap. This can either be due to a high rate of primary particles or due to successors of a primary avalanche produced by photo electric effect. Unabsorbed UV-photons emitted by a preceding avalanche can knock electrons from the cathode surface up to a few $\mathrm{mm}$ in radial direction from that avalanche. These electrons generate succeeding avalanches which at a later stage can transform into a streamer. Experimentally one observes precursors corresponding to the primary avalanche and then, with some delay, a current pulse with up to $100 \mathrm{~ns}$ delay [91], corresponding to the propagation of the streamer (See Fig 2.20). 


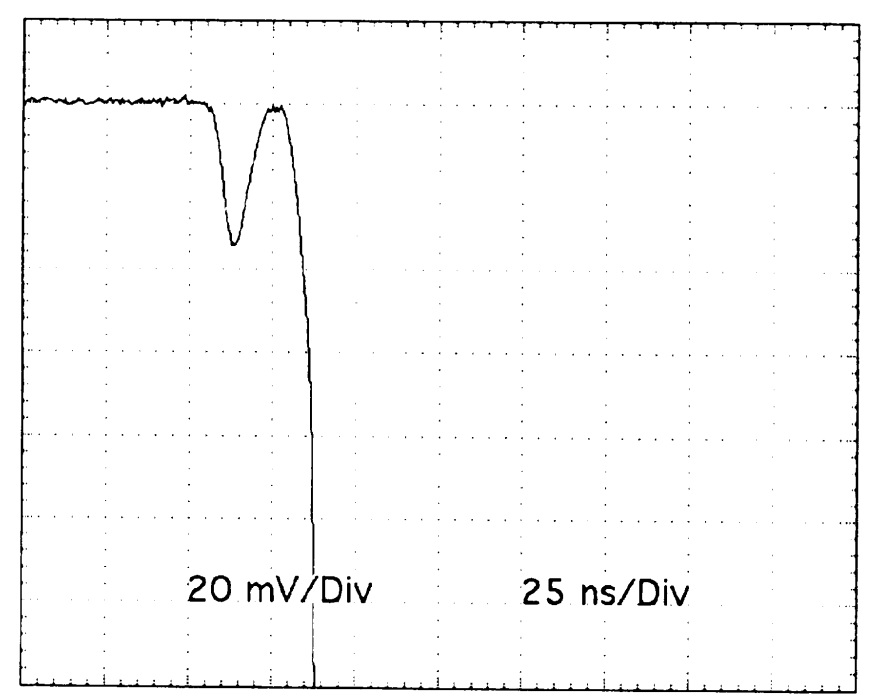

Figure 2.20: A measured voltage pulse in an RPC [40]. The first pulse corresponds to an avalanche (the precursor signal) and is followed by a streamer signal.

2. A rapid mechanism which converts directly the first avalanche into a streamer (Kanalaufbau).

There exist naturally variations of these two mechanisms, which can be understood as transitions between the two. Experimentally one often observes that streamer signals are preceded by a smaller pulse corresponding to an avalanche (the precursor signal), as in Fig. 2.20. At low voltages this behaviour is not detected, but if the voltage is increased, the streamer pulses occur with a time delay getting smaller towards higher voltages. Finally the precursor and streamer signals merge at a certain voltage [92].

In streamer-mode RPCs the appearance of streamers is desired, because the large streamer pulses need no amplification which simplifies the read out. In avalanchemode RPCs streamers are an undesired side-effect that worsens the detectors rate capability, because the amount of released charge in a streamer eventually enters the resistive electrodes and is much larger than in the case of a usual avalanche. Moreover, streamers produce a high read out strip multiplicity due to the low discrimination threshold that is required by the avalanche mode. The addition of $\mathrm{SF}_{6}$ in the $1 \%$ range suppresses strongly the appearance of streamers [39].

Due to the photonic origin of the phenomenon of streamers, the avalanche-tostreamer transition can not be studied with the detector physics described so far in this chapter. It requires to include gas self-photo-ionization in the model and it requires the knowledge of various photon emission and absorption cross sections for all gas constituents and for the detector materials. This kind of approach goes beyond 
the scope of this thesis. However, streamer breakdown in parallel plate detectors was reproduced by a quantitative model where short distance gas self-photo-ionization is included [93]. In that model, the photo-ionization in the backward region of increased electric field strength, as it is visible for example in Fig. 1.1, leads to a propagation of the ionization region in the cathode direction. Apart from this so called cathode streamer, the model also reproduces the anode streamer, where the field distortion in the forward region of the avalanches leads to an ionization wave in the anode direction. The occurrence of a precursor signal is also reproduced. The time interval between the precursor and the streamer decreases, as observed in experiment.

\subsection{Summary}

The important parameters to describe the generation and evolution of avalanches in RPCs are

- the average distance between primary clusters $\lambda$,

- the probability distribution for the number of electrons per cluster,

- the Townsend coefficient $\alpha(E / p)$,

- the attachment coefficient $\eta(E / p)$,

- the drift velocity $v_{D}(E / p)$ of electrons in the gas,

- the transverse and longitudinal diffusion coefficients $D_{T}(E / p)$ and $D_{L}(E / p)$,

- the potential of a point charge in a three layer geometry like the RPC and

- the value of the $z$-component of the weighting field in the central layer of this geometry.

The values of $\lambda$ and the cluster size distribution can be calculated with the program HEED. The Townsend and attachment coefficients, the drift velocity and the diffusion coefficients are functions of the electric field strength $E$ and the gas pressure $p$. These functionalities are obtained by the programs MAGBOLTZ and IMONTE.

The fundamental physical effect that leads to the deposit of free charge carriers in the gas gap of an RPC is the primary ionization of the gas atoms by the incident particle. The distance between the primary clusters is exponentially distributed around the mean value $\lambda$. The number of clusters in a gap of width $g$ is Poisson distributed around a mean of $\bar{n}=g / \lambda$. The maximum efficiency of an RPC is given by $\epsilon_{\max }=$ $1-\exp (-\bar{n})$, where $\exp (-\bar{n})$ is the probability to find no cluster in the gas gap. $\epsilon_{\max }$ is depending strongly on the used gas and the gap width $g$. The number of electrons 
per cluster follows a distribution that has a mean of a few electrons but a long tail to large electron numbers.

In a uniform electric field the propagation of an electron cloud can be described by a diffusion motion and a superposed constant drift motion. The diffusion follows a Gaussian distribution with a standard deviation that is depending on the diffusion coefficients and that is increasing with time. The longitudinal and transverse diffusion coefficients are in general not equal.

For the avalanche fluctuations we follow a model by W. Legler that describes the statistics of electron avalanches in electro negative gases at high electric fields and at large gas gain. The distribution depends on the values of $\alpha$ and $\eta$ explicitly. Since $\alpha$ and $\eta$ depend on the electric field and since this field can be influenced by the charge carriers of the avalanche (the space charge), we give analytic formulas for the potential of a point charge in an infinite plane condenser with three homogeneous layers. We find that this potential can be approximated well by the potential of a free charge and that of one mirror charge that is situated in the nearer electrode. This solution can be used to calculate the space charge field.

The induced current $i(t)$ of $N(t)$ unit charges moving with velocity $\vec{v}_{D}(t)$ at time $t$ is calculated using the weighting field formalism: $i(t)=\vec{E}_{w} \cdot \vec{v}_{D}(t) e_{0} N(t)$, where $\vec{E}_{w}$ is the weighting field and $e_{0}$ is the unit charge. Analytic formulas for the weighting field of a strip electrode in an RPC have been given.

The phenomenon of streamers can not be explained by our model, because we do not include any photonic effects. 


\section{Chapter 3}

\section{Monte Carlo Avalanche Simulation}

The Monte-Carlo simulation of the physical processes in particle detectors is an important tool for understanding the behaviour of the detectors in the particle physics experiments, in which they are or will be implemented. In order to optimize the detector physics parameters like gas mixture, gas pressure, gas gain and electronics parameters like preamplifier peaking time, noise, threshold settings etc., generally a detailed simulation of the detector response is carried out. For the simulation of wire and drift chambers one often utilizes the simulation tool GARFIELD [94]. For the simulation of RPCs no such tool exists. As a consequence, many experimental results have not been properly studied. Even though the geometry of the device is much simpler than that of a wire based detector, there are still disagreements about the explanation of several aspects of the performance of RPCs [7]. Thus the need for a detailed Monte-Carlo simulation of avalanches in RPCs arose. In this chapter we present four Monte-Carlo avalanche simulation programs that base the knowledge gathered in chapter \&. They are written in $\mathrm{C} / \mathrm{C}++$ and make use of the ROOT [95] data analysis framework.

The 1-D model: The first program is a one dimensional (1-D) simulation of the longitudinal avalanche development along the $z$-axis, which is divided into several steps. This model is described in detail in [1]. Saturation of the number of avalanche charges is implemented in a crude way by cutting the avalanche growth at a certain size. Diffusion is not implemented. The read out electronics are included and the program is used for fast and detailed studies of time resolutions and efficiencies of RPCs. It is described in section B.T.

The 1.5-D model: The program described in [1] was extended by an implementation of the space charge effect. The $z$-component of the electric field of the space charge is calculated dynamically and added to the applied field. With that the gas parameters like Townsend and attachment coefficient at each position and at each time step are calculated. The model is called "1.5-D" since the propagation of the avalanche charges is simulated only in one direction but the charge carriers 
at each position are assumed to be contained inside radial charge distributions with a width closely connected to the transverse diffusion coefficient. It is used for detailed studies of avalanche saturation, charge spectra, intrinsic charge-time correlations and the influence of the space charge effect on the time resolution. It is described in detail in section 3.2.

The 2-D model: The third program is a two dimensional avalanche simulation (2-D) where also the transverse spread of the avalanche due to the electric field contributions by the avalanche charges is taken into account. Cylindrical symmetry of the avalanche is assumed and the gas gap is divided into a two dimensional grid of the radial and longitudinal coordinates $r$ and $z$. The program allows the very detailed simulation of single avalanches but it is time consuming. It is described in detail in section 3.3.

The 3-D model: A three dimensional avalanche simulation (3-D) is presented in section 3.4. Here the gas gap is divided into a grid of the coordinates $x, y$ and $z$. We shall see that for the precise study of avalanches the segmentation has to be very fine which makes the program extremely time-consuming.

\subsection{The 1-D Model}

To calculate the final avalanche charge of a random avalanche in an RPC, the probability distributions from Eqs. 2.14, 2.17, 2.19 can be used. This is done by drawing random numbers according to Eqs. 2.21, 2.22, 2.23. In practice one is more interested in the signal development, i.e. the induced current at each time. As an example we will now follow the avalanche development for a single initial electron starting at one edge of the gas gap. We divide the gas gap into $N$ steps of size $\delta z$. The average multiplication of a single electron over this distance is given by $\bar{n}(z)=\exp ((\alpha-\eta) \delta z)$. Starting with one electron at $z=0$, we find $n_{1}$ electrons at $z=\delta z$, where $n_{1}$ is from Eqs. 2.21, 2.22, 2.23. Each of these electrons will again multiply the same way. To find the number $n_{2}$ of electrons at $z=2 \delta z$ we loop over the $n_{1}$ electrons, draw a number from Eqs. 2.21, 2.22, 2.23 for each electron and sum them up. This procedure can be repeated through the whole gap, but it is very time consuming. If the number of electrons $n_{i}$ at a given position $i \delta z$ is sufficiently large ( $\left.\gtrsim 150\right)$, we can use the central limit theorem and calculate the new number of electrons by drawing a random number from a Gaussian distribution with mean $\mu$ and sigma $\sigma_{\mu}$, that are given by

$$
\mu=n_{i} \bar{n}(z) \text { and } \quad \sigma_{\mu}=\sqrt{n_{i}} \sigma(z)
$$

where $\sigma(z)$ is from Eqs. 2.16, 2.18, 2.20. This makes the simulation procedure very fast. Fig. 3.1 shows examples of individual avalanches starting from a single electron. The very beginning of the avalanche decides on the final avalanche size. 


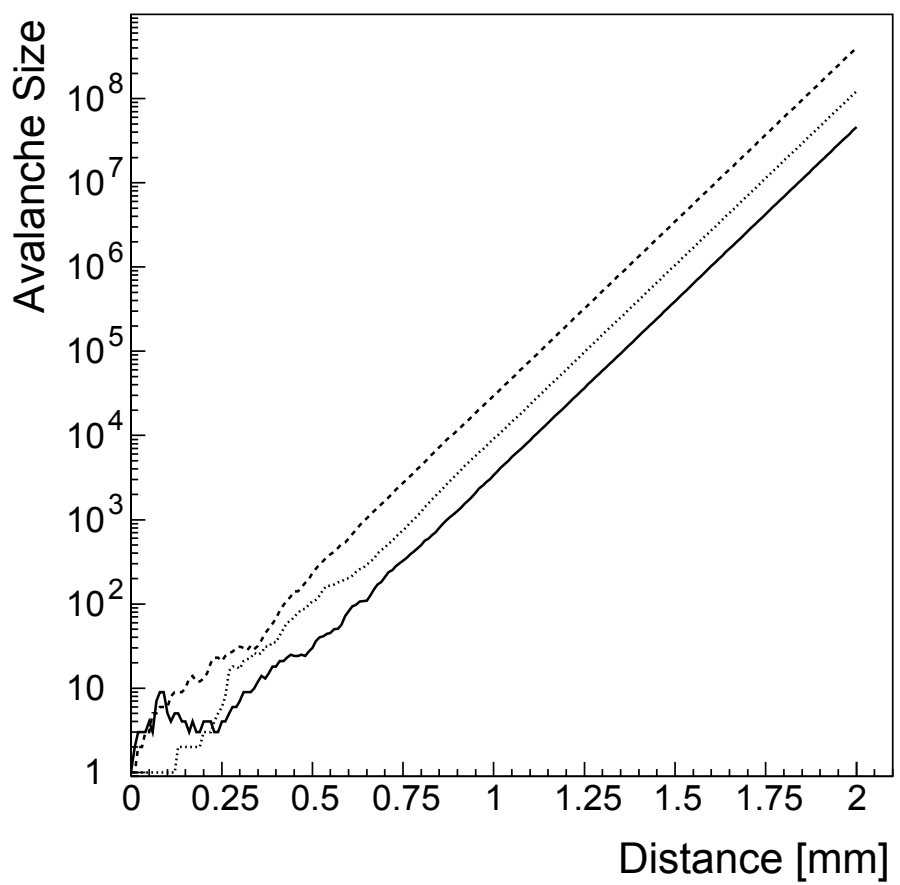

Figure 3.1: Avalanches started by a single electron at $z=0$ for $\alpha=13 / \mathrm{mm}, \eta=$ $3.5 / \mathrm{mm}$ [1]]. We see that the very beginning of the avalanche decides on the final avalanche size. Once the number of electrons is sufficiently large, the avalanche grows like $\exp ((\alpha-\eta) z)$

The 1-D model is described in detail in [1]. Its basic structure is the following:

1. The gas gap (the $z$-axis) is divided into $N$ steps of size $\delta z=g / N$ corresponding to time steps of $\delta t=\delta z / v_{0}$, where $v_{0}=v_{D}\left(E_{0} / p\right)$ is the electron drift velocity from Fig. 2.4 at the applied electric field strength $E_{0}$ and at the pressure $p$.

2. We assume that all particle tracks are perpendicular to the electrode plates of the detector.

3. The primary clusters are distributed onto the steps, with distances following an exponential distribution with a mean taken from Fig. 2.2. The first cluster is put at a distance from the cathode, that is obtained by drawing a random number from an exponential distribution with a mean equal to the mean free path. The second cluster is put at a distance from the first cluster, that is calculated accordingly. This procedure is repeated until the anode is reached.

4. Primary electrons are put to each cluster, following the cluster size distribution from Fig. 2.3. 
5. The drift velocity $v_{0}=v_{D}\left(E_{0} / p\right)$, the Townsend coefficient $\alpha\left(E_{0} / p\right)$ and the attachment coefficient $\eta\left(E_{0} / p\right)$ for the applied electric field $E_{0}$ and the pressure $p$ are taken from Figs. 2.4 and 2.8 .

6. The avalanches for each single electron are simulated using Eqs. 2.21, 2.22, 2.23 and the procedure that is outlined at the beginning of this section. This provides $N(t)$, the total number of electrons at time $t$.

7. If $N(t)$ exceeds a certain total number of electrons $N_{\text {sat }}$, the avalanche growth is stopped and the $N_{\text {sat }}$ electrons are propagated towards the anode. This procedure simulates the space charge effect.

8. At each time step, the current induced by the drifting electrons is calculated. The $N_{j}(t)$ electrons that are propagated from step $j$ to step $j+1$ induce the current $i(t)=E_{w} v_{0} e N_{j}(t)$ (see Eq. 2.33). The induced currents of the electrons at the different steps are summed up.

9. Steps 6 to 8 are repeated until all electrons have left the gas gap.

10. The obtained current signal is convoluted with the amplifier delta response $f(t)$ [1]

$$
h(s)=\frac{n^{-n} e^{n} n ! \tau}{(1+s \tau)^{n+1}} \quad \rightarrow \quad f(t)=\mathcal{L}[h(s)]=n^{-n} e^{n}\left(\frac{t}{\tau}\right)^{n} e^{-\frac{t}{\tau}}
$$

where $t_{p}=n \tau$ is the peaking time and $n$ corresponds to the number of stages. Noise is included by adding a value drawn from a Gaussian distribution to the signal in each time bin with a standard deviation giving the correct Equivalent Noise Charge (ENC) at the output.

\subsection{The 1.5-D Model}

In this section we extend the 1-D simulation model described in section 3.1 by including diffusion and space charge effects. The basic structure of the simulation is the following:

1. The gas gap (the $z$-axis) is divided into $N$ steps of size $\delta z=g / N$ corresponding to time steps of $\delta t=\delta z / v_{0}$, where $v_{0}=v_{D}\left(E_{0} / p\right)$ is the electron drift velocity from Fig. 2.4 at the applied electric field $E_{0}$.

2. We assume that all particle tracks are perpendicular to the electrode plates of the detector. 
3. The primary clusters are distributed onto the steps, with distances following an exponential distribution with a mean taken from Fig. 2.2. The procedure is the same as in the 1-D model.

4. Primary electrons are put to each cluster, following the cluster size distribution from Fig. 2.3.

5. The electric field $E(z)$ at all steps where electrons are situated is calculated. Here we also include transverse diffusion. The procedure is described in detail in sections 3.2 .2 and 3.2 .3 .

6. The drift velocity $v_{D}(E(z) / p)$, the Townsend coefficient $\alpha(E(z) / p)$ and the attachment coefficient $\eta(E(z) / p)$ are calculated at each step where electrons are found.

7. The avalanches for each single electron are simulated using Eqs. 2.21, 2.22, 2.23 and the procedure outlined in section 3.1. We also include longitudinal diffusion and the charges are redistributed onto the steps following the procedure that is described in section 3.2 .1 .

8. At each time step, the current and charge induced by the drifting electrons are calculated according to Eqs. 2.34 and 2.41. The procedure is described in more detail in section 3.2 .7 .

9. Steps 5 to 8 are repeated until all electrons have left the gas gap.

\subsubsection{Longitudinal Diffusion}

In section 2.2.2 we discussed longitudinal diffusion. If an electron cloud drifts from position $z$ to position $z+\delta z$, there is a certain probability for each electron to diffuse to a position different from $z+\delta z$. Since we assume that the diffusion is strictly Gaussian, the probability distribution is given by Eq. 2.9a. Thus the new $z$-coordinate for each electron can be calculated by drawing a random number from a Gaussian distribution with a mean $z+\delta z$ and a standard deviation $\sigma=D_{L} \sqrt{\delta z}$. Fig. 3.2 shows two example simulated avalanches with longitudinal diffusion.

The longitudinal diffusion has an influence on the average avalanche growth. As an example we consider an avalanche started by two electrons and assume that the avalanches grow exponentially. Then the average number of electrons grows like $n(z)=2 e^{\alpha z}$. We compare this to the average number of electrons of two avalanches that travelled the distances $z+\delta z$ and $z-\delta z: n(z, \delta z)=e^{\alpha(z+\delta z)}+e^{\alpha(z-\delta z)}$. The ratio of the two is:

$$
\frac{e^{\alpha(z+\delta z)}+e^{\alpha(z-\delta z)}}{2 e^{\alpha z}}=\frac{e^{\alpha \delta z}+e^{-\alpha \delta z}}{2}=\cosh (\alpha \delta z)
$$



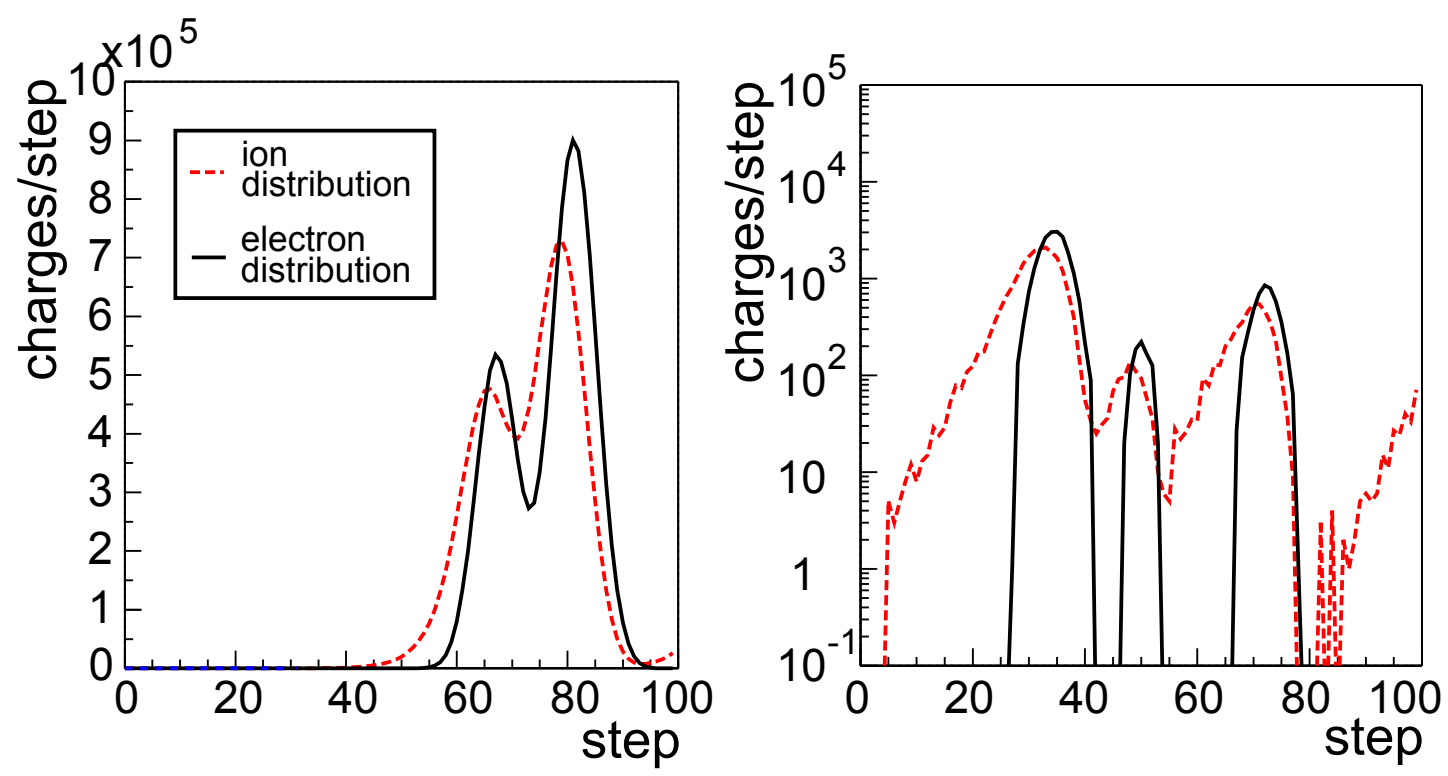

Figure 3.2: Snapshots of simulated avalanches with longitudinal diffusion. A $0.3 \mathrm{~mm}$ gas gap is divided in 100 steps. The right image has a logarithmic scale.

The function cosh has a minimum at $\delta z=0$ where its value is 1 . This shows that longitudinal diffusion generally increases the avalanche charge.

\subsubsection{Transverse Diffusion}

The distribution of charges in an avalanche grows transversely due to diffusion as the avalanche propagates (section 2.2.2). Considering an avalanche propagating along the $z$-axis in the gas gap, we assume that

- the avalanche has rotational symmetry,

- the transverse charge distribution is given only by transverse diffusion and

- the diffusion is Gaussian.

Then the normalized radial charge distribution on a disc perpendicular to the $z$-axis is given by Eq. 2.90

$$
\varphi_{T}\left(r, l\left(z^{\prime}\right)\right)=\frac{1}{D_{T}^{2} l\left(z^{\prime}\right)} \exp \left(-\frac{\left(r-r_{0}\right)^{2}}{2 D_{T}^{2} l\left(z^{\prime}\right)}\right) .
$$


Here $l\left(z^{\prime}\right)$ is the distance drifted by the electrons from the position of the generation of the primary cluster to the position of the disc at $z^{\prime}$. Since the distribution is centered around the $z$-axis, we choose $r_{0}=0$.

\subsubsection{Space Charge Effect}

An analytic solution for the potential $\Phi\left(r, \phi, z, r^{\prime}, \phi^{\prime}, z^{\prime}\right)$ of a point charge in an infinite plane condenser comprising three homogeneous layers is given by Eq. 2.26. Here $(r, \phi, z)$ is the point of observation and $\left(r^{\prime}, \phi^{\prime}, z^{\prime}\right)$ the position of the point charge. We use this solution to calculate the electric field of the charges in the gas gap (of the space charge) at each position and time. Since the simulation is performed only along the $z$-axis, it is sufficient to use the potential only at $(r=0, \phi=0, z)$ and we write $\Phi\left(r=0, \phi=0, z, r^{\prime}, \phi^{\prime}, z^{\prime}\right)=\Phi\left(z, r^{\prime}, \phi^{\prime}, z^{\prime}\right)$. The $z$-component of the electric field of the point charge is found by derivation, similar to Eq. 2.29c, as

$$
E_{z}\left(z, r^{\prime}, \phi^{\prime}, z^{\prime}\right)=-\frac{\partial \Phi}{\partial z}\left(z, r^{\prime}, \phi^{\prime}, z^{\prime}\right)
$$

The avalanche charge is assumed to be contained in a disc perpendicular to the $z$ axis. The radial charge distribution $\varphi_{T}\left(r, l\left(z^{\prime}\right)\right)$ at each $z$-position is given by Eq. 3.2. Then the electric field $\bar{E}_{z}\left(r=0, \phi=0, z, l, z^{\prime}\right)=\bar{E}_{z}\left(z, l, z^{\prime}\right)$ of a disc containing the unit charge at position $z$ is calculated as the integral over Eqs. 3.2 and 3.3 as

$$
\bar{E}_{z}\left(z, l, z^{\prime}\right)=-\int_{0}^{\infty} \varphi_{T}\left(r, l\left(z^{\prime}\right)\right) \frac{\partial \Phi\left(z, r^{\prime}, \phi^{\prime}, z^{\prime}\right)}{\partial z} r^{\prime} d r^{\prime}
$$

The $\phi^{\prime}$-integration has already been carried out earlier, when we assumed rotational symmetry in $\varphi_{T}\left(r, l\left(z^{\prime}\right)\right.$ ) (section 2.2.2). The positions of the charge distribution and the point of observation are shown schematically in Fig. 3.3. While the electric field of a point charge following Eq. 3.3 diverges at $\left(r \rightarrow r^{\prime}, \phi \rightarrow \phi^{\prime}, z \rightarrow z^{\prime}\right)$, the field following Eq. 3.4 is well defined everywhere. This is shown in Fig. 3.4, where the potential of a unit point charge (given by Eq. 2.26) is compared to the potentials of the Gaussian charge distribution and of a uniform charge distribution.

The field $E_{z}(z)$ of all the charge in the gap (the field of the space charge) is calculated by integration (summation) over all the discs.

$$
\begin{aligned}
E_{z}(z) & =\int_{0}^{g} q\left(z^{\prime}\right) \bar{E}_{z}\left(z, l, z^{\prime}\right) d z^{\prime} \\
& \approx \sum_{m=0}^{N} q_{m} E_{z}\left(z, l_{m}, z_{m}^{\prime}\right) .
\end{aligned}
$$




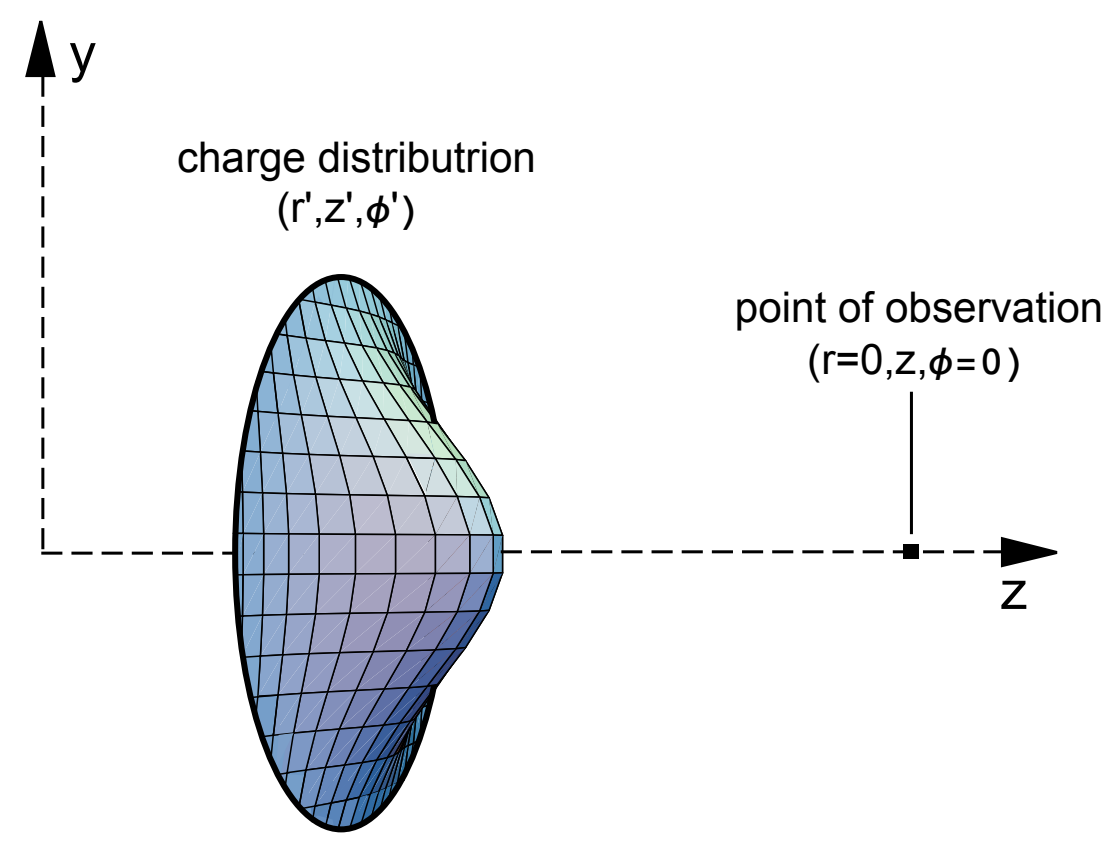

Figure 3.3: The geometry for the 1.5-D simulation. The point of observation is $(r=$ $0, \phi=0, z)$ and the disc with the Gaussian radial charge distribution is positioned at $z^{\prime}$.

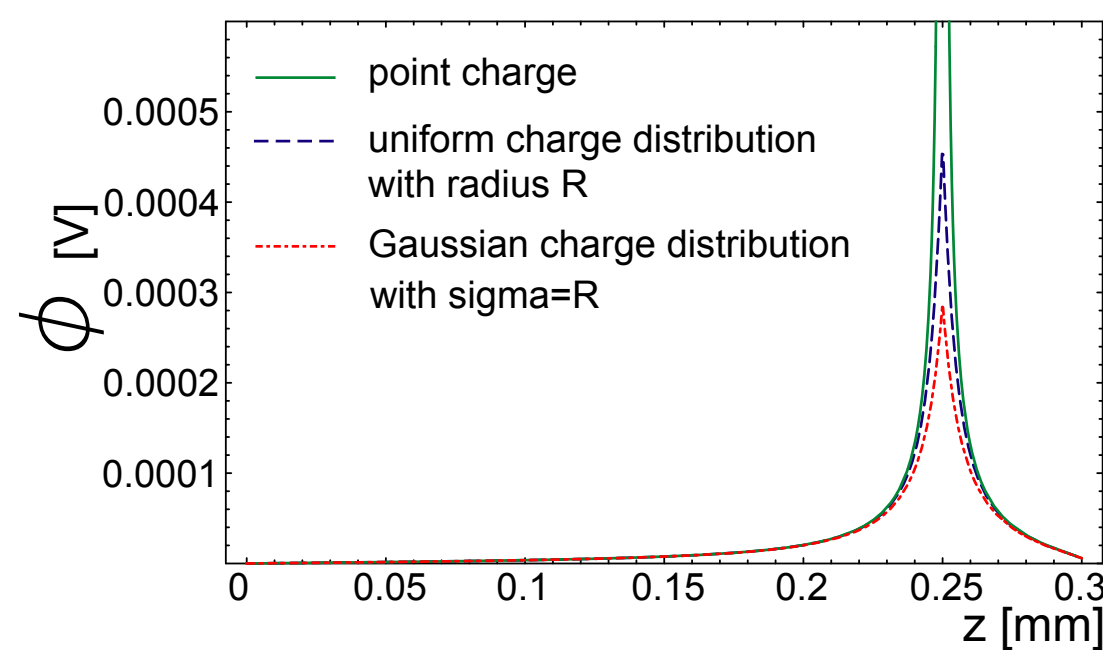

Figure 3.4: A comparison of the potentials of a point charge (Eq. 2.26) and two different transverse charge distributions in a three layer geometry like the RPC across a $0.3 \mathrm{~mm}$ gap. The first charge distribution is uniform with radius $R=6 \mu \mathrm{m}$, the second is Gaussian with the standard deviation $\sigma=R$. Moreover we used $\varepsilon_{1}=\varepsilon_{3}=8 \varepsilon_{0}$, $\varepsilon_{2}=\varepsilon_{0}, P=0, z^{\prime}=0.25, g=0.3, q=2$ and $p=2.3$. 

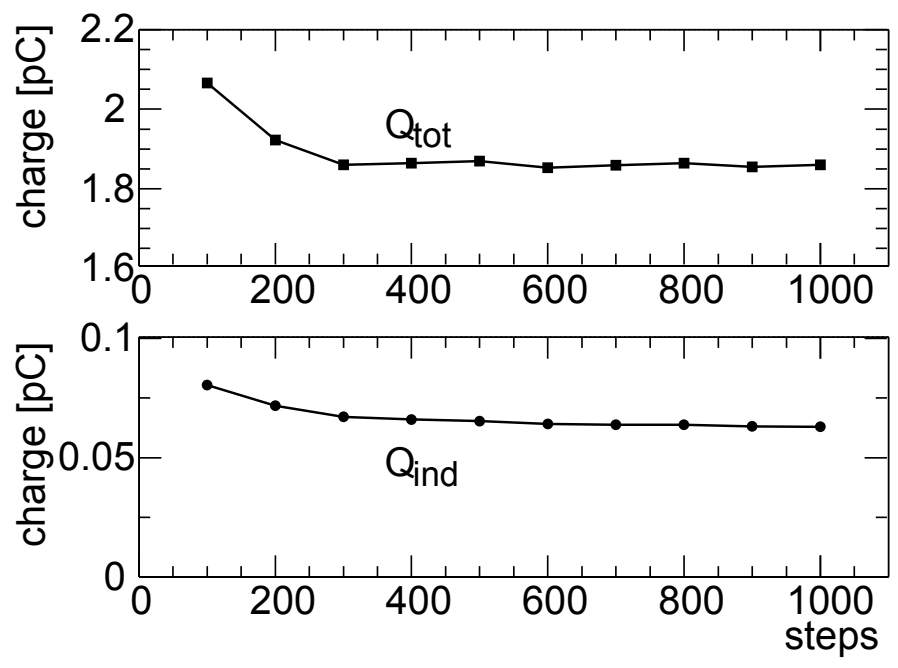

Figure 3.5: The average signal charge $Q_{t o t}$, which is the charge of the positive ions in the gas gap at the end of the avalanche development, and the induced charge $Q_{\text {ind }}$ (see Eq. 2.41) for different numbers of steps. We used a $0.3 \mathrm{~mm}$ gap timing RPC with $\mathrm{HV}=2.7 \mathrm{kV}$ and average avalanches. For a number of steps larger than 200 we find a fluctuation of $2.2 \%$ and $0.3 \%$ r.m.s. for $Q_{i n d}$ and $Q_{t o t}$.

In Eq. $3.5 b$ we moved from the continuous to a discrete system. Here $q_{m}$ is the charge in the step $m$, which drifted the distance $l_{m}$ from the position of the formation of the primary cluster to the current position $z_{m}^{\prime}$. We can now calculate the field of the space charge in the gas gap at all positions. Above a certain step number the calculation is only very slightly depending on the chosen step size, which is shown in Fig. 3.5. In the program an adequate number of values of $\bar{E}_{z}\left(z, l, z^{\prime}\right)$ for different $z, l$ and $z^{\prime}$ is memorized in a three dimensional table for computational efficiency reasons and the values for are obtained during the simulation by interpolation.

At this point we would like to mention that the transverse dispersion of the avalanche is simulated only with regard to diffusion. We assume that the transverse diffusion coefficient $D_{T}$ is constant while in reality $D_{T}$ depends on the electric field. In Fig. 3.6 we show a comparison of the $z$-components of the electric fields of two transverse Gaussian charge distributions with different standard deviations $\sigma$. The $\sigma$ differs by $20 \%$, leading to a derivation in the value of the electric field of up to $30 \%$. Moreover, the repulsion of the charges of same sign will also contribute to the transverse dispersion, especially in the final stage of the avalanche, where a strong space charge effect is present. This means that the radial charge distribution will not be Gaussian at that stage. Since these effects are not included in the simulation, the radial charge density might be overestimated which can further lead to an overestimation of the longitudinal space charge effect. A detailed discussion to this topic is found in a later chapter (section 7.1). 


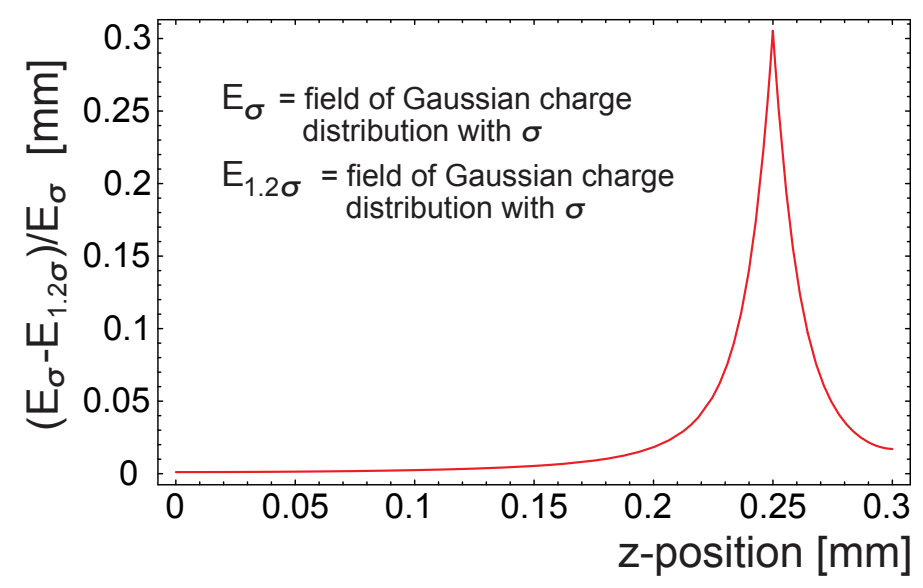

Figure 3.6: A comparison of the $z$-component of the electric field of two transverse Gaussian charge distributions with different standard deviations $\sigma$. The charges are at $z=0.25 \mathrm{~mm}$ in a $0.3 \mathrm{~mm}$ gap. The $\sigma$ of one Gaussian is $20 \%$ higher than the other, leading to a reduction of the electric field of up to $30 \%$ close to the charges.

\subsubsection{Electrons in the Anode Resistive Layer}

Electrons that reach the anode will leave the gas gap and enter the anode. If it is made of a conducting material, the charges disappear instantly. If the anode is made of a resistive material, a 'relaxation time' $\tau$ is needed for the charges to drain off (See section 1.3.4). $\tau$ is several orders of magnitude larger than the signal time which leads to an accumulation of electrons at the surface of the resistive anode. These charges can have a strong influence on the field in the gas gap, especially on the field close to the anode, so they have to be included in the simulation. For the potential we use Eq. 3.4 at $z^{\prime}=g: \bar{E}_{z}\left(z, l, z^{\prime}=g\right)$. The charge is again distributed in a transverse Gaussian with the standard deviation $\sigma$ as before depending on the transverse diffusion coefficient and the distance $l$ that the cluster of electrons has drifted from the point of its creation to the anode.

\subsubsection{Field Dependence of the Electron Multiplication}

As was mentioned frequently, the dependence of the multiplication coefficients $\alpha(E / p)$ and $\eta(E / p)$ on the electric field $E$ leads to saturation of the avalanche growth as soon as the size of the avalanche is sufficiently large so that the charge carriers disturb the applied external field. The growth is then not exponential, it becomes approximately linear. Fig. 3.7b shows three example avalanches that were started by single electrons at the cathode of a $0.3 \mathrm{~mm}$ gap and that propagate under the influence of the space charge effect. We see that even though the initial growth of the avalanches differs a lot, the induced current at the final stage becomes similar. 

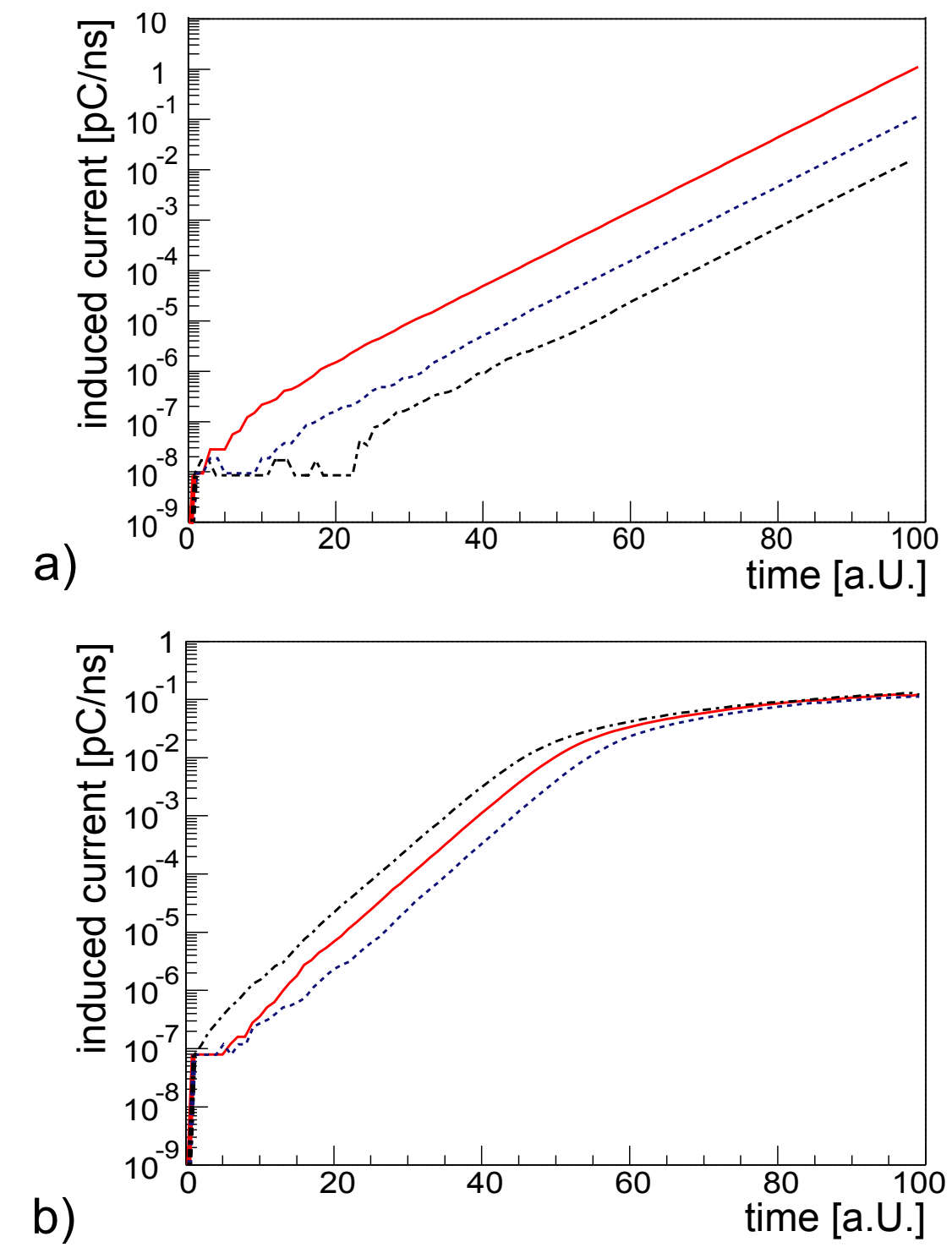

Figure 3.7: A comparison of the current induced by three avalanches that started with one electron at $z=0$. A $0.3 \mathrm{~mm}$ gap is divided in 100 steps, corresponding to time steps of around 14 ps. a) We chose a low value for the applied electric field. Thus the gain is quite low. The space charge effect is switched off in the simulation. b) The space charge effect is switched on. The gain is larger than in a). We observe a clear saturation effect. 
Fig. 3.8 shows an example of an avalanche entering the space charge regime.

(a) In the first figure $0.58 \mathrm{~ns}$ have passed since the passage of the ionizing particle. One electron cluster has already reached the anode. The field in front of the anode is lowered by the electrons in the resistive anode surface and by the positive ions in front of it. At the tip and the tail of the remaining cluster the electric field is increased by $\approx 15 \%$. At the center of the electron cloud the field is about $25 \%$ lower (compare to Fig. 1.1).

(b) In the second image at $0.77 \mathrm{~ns}$ the field in the center of the electron cloud is lowered to an extent that pushes the effective Townsend coefficient to negative values. This leads to strong attachment of electrons, generating many negative ions.

\subsubsection{Field Dependence of the Drift Velocity}

The repulsive or attractive electric fields of the avalanche charge carriers lead to a longitudinal spread of the electron distribution of an avalanche. The fundamental parameter describing this effect is the drift velocity, which depends on the gas pressure and the electric field: $v_{D}(E / p)$. To implement the longitudinal space charge effect in the simulation we calculate at each step $m$, where we find electrons, the drift velocity $v_{D}\left(E_{m} / p\right)$, where $E_{m}$ is the electric field at the step $m$ and compare this drift velocity to the drift velocity $v_{0}=v_{D}\left(E_{0} / p\right)$ at the applied electric field $E_{0}$. As an example let us consider 100 electrons at step $m$ and a calculated drift velocity of $1.5 v_{0}$. Then we put 50 electrons to step $m+1$ and 50 to step $m+2$. Accordingly, if we calculate a drift velocity of $0.9 v_{0}$, we put 90 electrons to step $m+1$ and 10 electrons stay at step $m$. In the program this is implemented by calculating

$$
\zeta=\frac{v_{D}\left(E_{m} / p\right)}{v_{0}} \quad \text { and } \quad p(\zeta)=\zeta-\operatorname{trunc}(\zeta)
$$

where 'trunc' means truncation of the decimals. The probability for an electron at step $m$ to drift to step $m+\operatorname{trunc}(\zeta)$ is $1-p(\zeta)$ while the probability to drift to step $m+1+\operatorname{trunc}(\zeta)$ is $p(\zeta)$. If $n$ electrons have to be distributed onto the two steps, we put trunc $(p(\zeta) n)$ electrons to step $m+1+\operatorname{trunc}(\zeta)$ and $n-\operatorname{trunc}(p(\zeta) n)$ electrons to step $m+\operatorname{trunc}(\zeta)$.

Fig. 3.9 shows two example simulated avalanches without longitudinal diffusion. Each electron cluster was generated in one step, the position of the formation of the primary cluster. At a later stage the electrons are distributed over more steps. The electrons at the tip of the electron distributions have a larger drift velocity, which is 

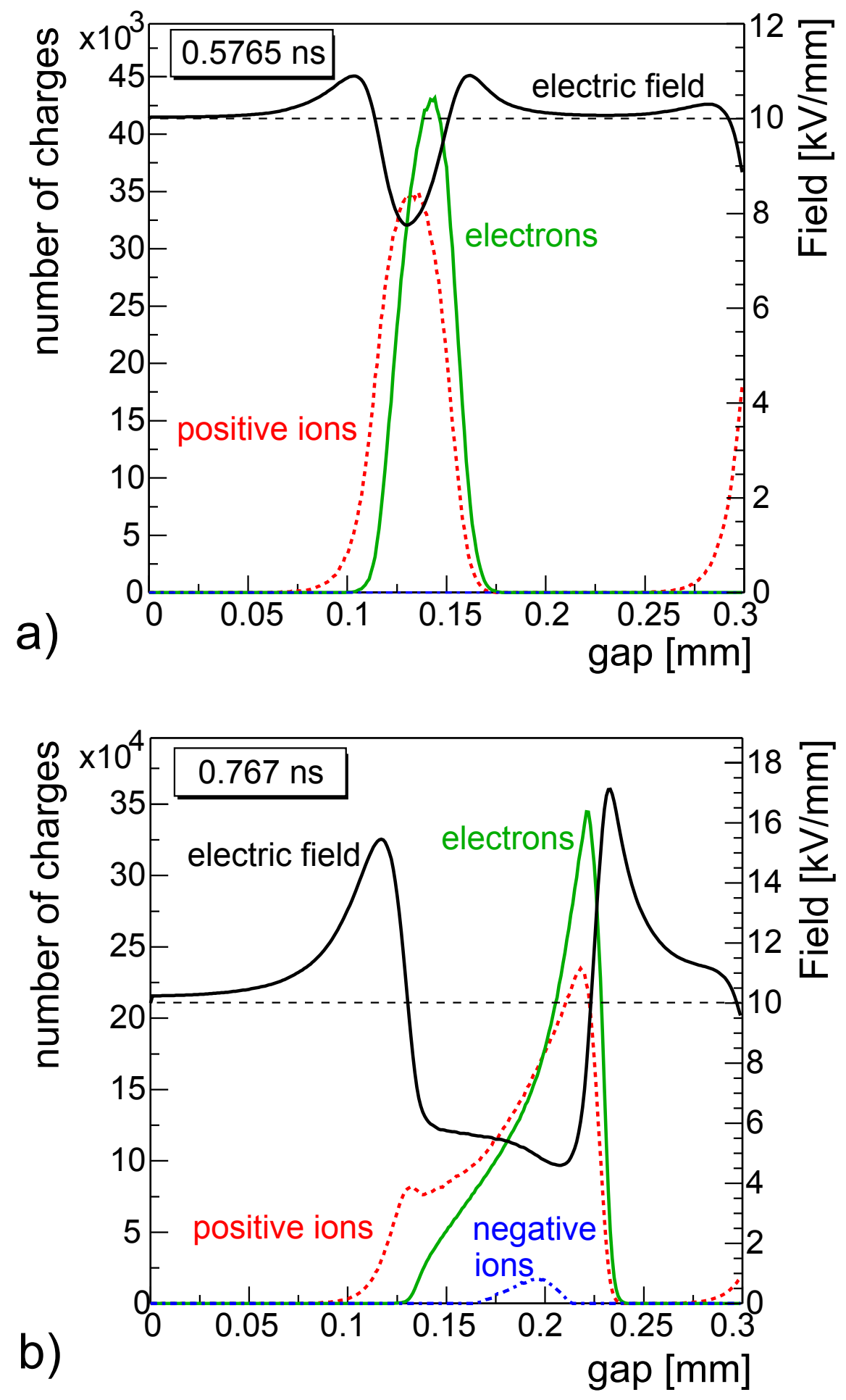

Figure 3.8: Snapshots of a simulated avalanche. A $0.3 \mathrm{~mm}$ gas gap is divided in 500 steps. The ion and electron distributions and the electric field $E_{z}(z)$ (Eq. 3.5b) are shown, corresponding to the left and right axes, respectively. We used the following values, which correspond to the geometry of a Timing RPC with a gap of $g=0.3 \mathrm{~mm}$ width: $\varepsilon_{1}=\varepsilon_{3}=8 \varepsilon_{0}, \varepsilon_{2}=\varepsilon_{0}, P=0, z^{\prime}=0.25, q=2$ and $p=2.3$. The high voltage is $3 \mathrm{kV}$, leading to an applied electric field of $10 \mathrm{kV} / \mathrm{mm}$. 

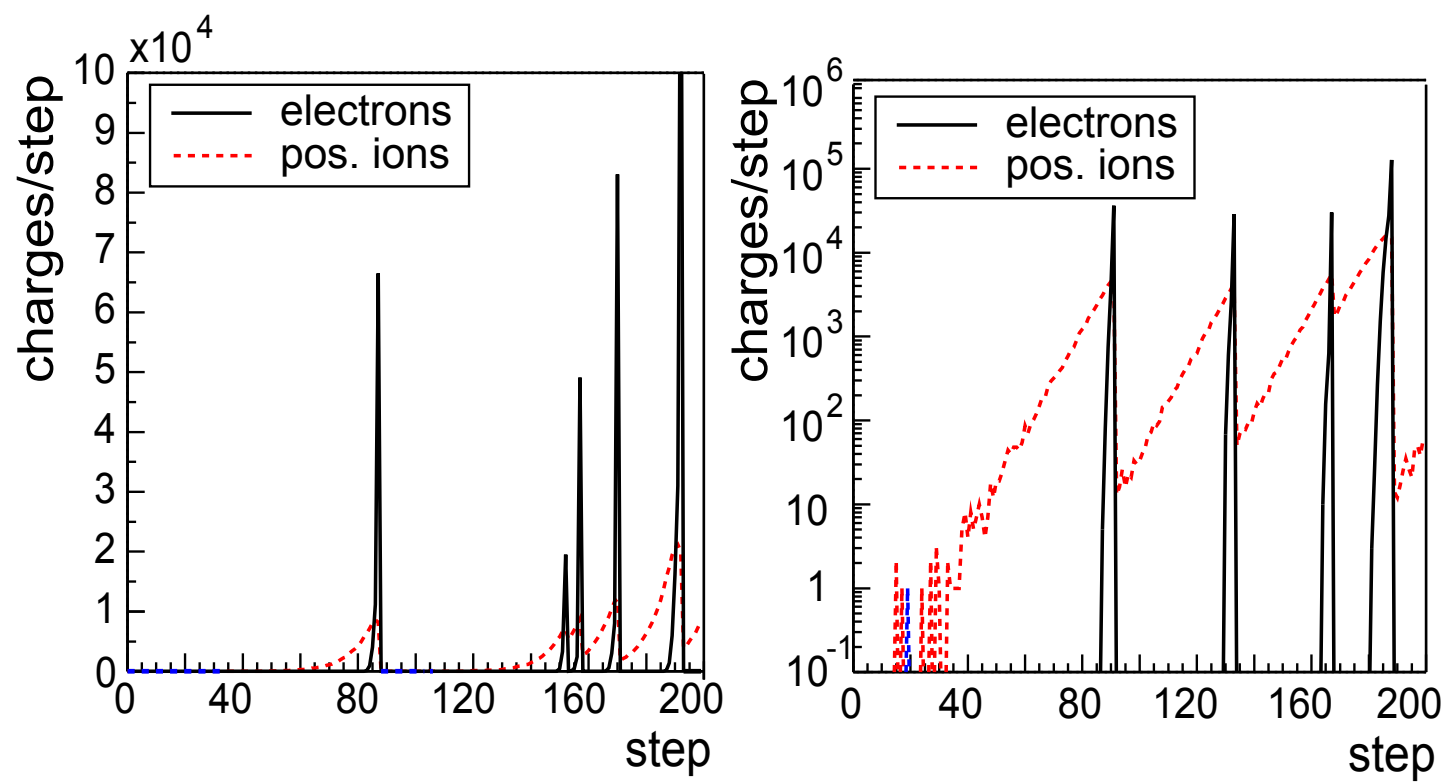

Figure 3.9: Snapshots of simulated avalanches without longitudinal diffusion. A $0.3 \mathrm{~mm}$ gas gap is divided into 200 steps. The right image has a logarithmic scale. The clusters are spread over a few steps due to the repulsive and attractive forces of the space charge.

due to the repulsive field generated by all the electrons behind. Other electrons, e.g. in the center of the electron clouds, have a lower drift velocity, due to the attractive force of the ions in their back and the repulsion by the electrons in front (compare to Fig. 1.1).

\subsubsection{Induced Current Signal and Induced Charge}

While the electrons are propagated through the gas, the currents induced by their motion are calculated to obtain the induced signal. Assuming $N_{j}(t)$ electrons are being propagated from step $j$ with the calculated velocity $v_{j}\left(E_{j} / p\right)$. Then the induced current is calculated using Eqs. 2.33 and 2.39 as $i_{j}=E_{z} v_{j}\left(E_{j} / p\right) e_{0} N_{j}(t)$. Here $E_{z}$ is the $z$-component of the weighting field and $e_{0}$ is the unit charge. The induced currents of the electrons at the different steps are summed up, giving the total induced current at the given time step. At the same time we calculate the induced charge. At each step $j$ the induced charge $q_{j}$ is connected to the induced current $i_{j}$ by

$$
q_{j}=\delta t i_{j}=\frac{\delta z}{v_{j}\left(E_{j} / p\right)} i_{j}=E_{z} e_{0} N_{j}(t) \delta z
$$


where $\delta t$ is the time step of the simulation and $\delta z$ is the step size. The induced charge at all time steps is summed up until all electrons have reached the anode and the signal development is finished. The induced current of the moving ions is not taken into account, since it is much smaller due to their small drift velocity.

\subsection{The 2-D Model}

We use cylindrical coordinates $r, z$ and $\phi$ and assume rotational symmetry of the avalanche around the z-axis. We simulate only avalanches started by one electron starting from a given position in the gas gap. The simulation routine has the following basic structure:

1. A cylindric volume of the gas gap is divided into a two dimensional grid of the $r$ and $z$-coordinates. If the $z$-coordinate is divided into $N_{z}$ steps of size $\delta z=$ $g / N_{z}$, the corresponding time steps of the simulation are $\delta t=\delta z / v_{D}\left(E_{0}, p\right)$, where $v_{D}\left(E_{0}, p\right)$ is the electron drift velocity from Fig. 2.4 at the applied electric field $E_{0}$. The $r$-coordinate is divided into $N_{r}$ steps of an appropriately chosen size $\delta r$. The charge that is situated in the grid point $\left(r^{\prime}, z^{\prime}\right)$ is actually a charge ring of size $\delta r$ and $\delta z$ centered at the $z$-axis (see Fig. 3.10).

2. One electron is put inside the volume.

3. A two dimensional electric field vector $\left(E_{z}, E_{r}\right)$ at each bin is calculated, if there is an electron in that bin.

4. The Townsend and attachment coefficients, the drift velocity and the diffusion coefficients at each bin are calculated.

5. The avalanches for each single electron are simulated using Eqs. 2.21, 2.22, 2.23 and the procedure outlined in section 3.1. Each electron is redistributed onto the bins. Here also longitudinal and transverse diffusion are included.

6. Steps 3 - 5 are repeated until all electrons leave the gas gap.

We assume a detector geometry as in [69, 96, 97], where only one electrode is made of a resistive material and the other one is made of aluminum. We assume that the conductive electrode is the anode and that the cathode is made of $3 \mathrm{~mm}$ thick glass. Due to the conductivity of the anode, the electrons entering the anode plate disappear instantly. They do not contribute to the electric field in the gas gap. 


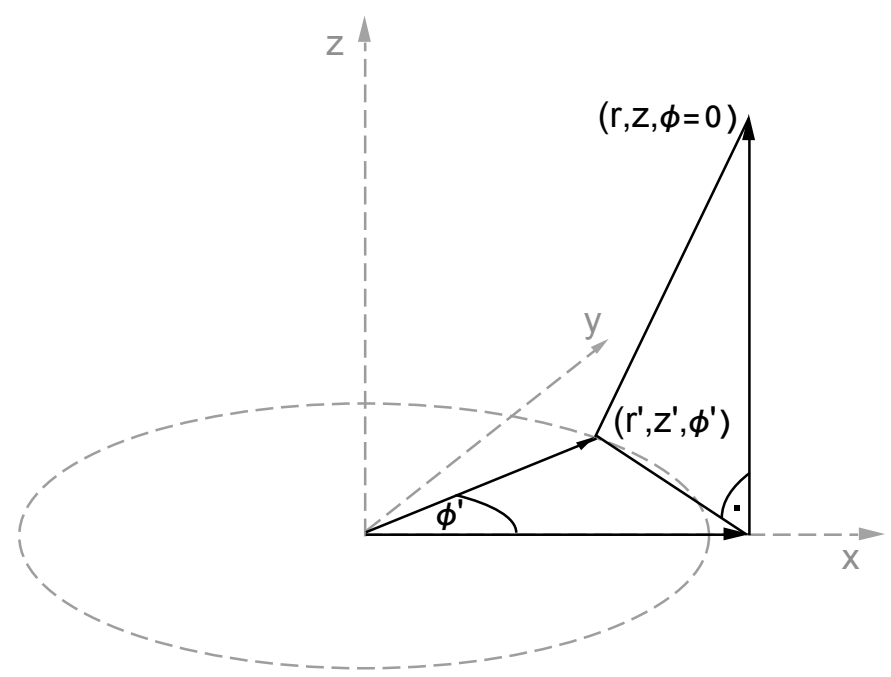

Figure 3.10: Geometry for calculating the electric field of a charge ring.

\subsubsection{Calculation of the Electric Field Vector}

In this section we give analytic formulas for the electric field of a charged planar ring of radius $r^{\prime}$ at $z^{\prime}$. We use the electric field solutions of a point charge in cylindrical coordinates] and choose $\phi=0$ (See Fig. 3.10).

$$
\begin{aligned}
& E_{r}\left(r, z, r^{\prime}, z^{\prime}\right) \approx \frac{Q}{4 \pi \varepsilon_{2}} \int_{0}^{2 \pi} \frac{r-r^{\prime} \cos \left(\phi^{\prime}\right)}{\left(P^{2}+\left(z-z^{\prime}\right)^{2}\right)^{\frac{3}{2}}} d \phi^{\prime} ; \\
& E_{\phi}\left(r, z, r^{\prime}, z^{\prime}\right) \approx \frac{Q}{4 \pi \varepsilon_{2}} \int_{0}^{2 \pi} \frac{-r^{\prime} \sin \left(\phi^{\prime}\right)}{\left(P^{2}+\left(z-z^{\prime}\right)^{2}\right)^{\frac{3}{2}}} d \phi^{\prime} ; \\
& E_{z}\left(r, z, r^{\prime}, z^{\prime}\right) \approx \frac{Q}{4 \pi \varepsilon_{2}} \int_{0}^{2 \pi} \frac{z-z^{\prime}}{\left(P^{2}+\left(z-z^{\prime}\right)^{2}\right)^{\frac{3}{2}}} d \phi^{\prime} .
\end{aligned}
$$

The value $P$ depends on $r, r^{\prime}$ and $\phi^{\prime}$ and is defined in Eq. 2.25. The solutions to Eqs. 3.7 are

\footnotetext{
${ }^{1}$ The Eqs. 2.30 to 2.32 give the electric field of a free charge and one mirror charge. We use the first of the two terms in each of those equations.
} 


$$
\begin{aligned}
& E_{r}\left(r, z, r^{\prime}, z^{\prime}\right) \approx \frac{Q}{4 \pi \varepsilon_{2}} \frac{2}{r a^{2} b}\left[c^{2} E\left(\frac{-4 r r^{\prime}}{b^{2}}\right)+a^{2} K\left(\frac{-4 r r^{\prime}}{b^{2}}\right)\right], \\
& E_{\phi}\left(r, z, r^{\prime}, z^{\prime}\right)=0 \\
& E_{z}\left(r, z, r^{\prime}, z^{\prime}\right) \approx \frac{Q}{4 \pi \varepsilon_{2}} \frac{4\left(z-z^{\prime}\right)}{a^{2} b} E\left(\frac{-4 r r^{\prime}}{b^{2}}\right) .
\end{aligned}
$$

where

$$
\begin{aligned}
& a^{2}=\left(r+r^{\prime}\right)^{2}+\left(z-z^{\prime}\right)^{2}, \\
& b^{2}=\left(r-r^{\prime}\right)^{2}+\left(z-z^{\prime}\right)^{2}, \\
& c^{2}=r^{2}-\left(r^{\prime}\right)^{2}-\left(z-z^{\prime}\right)^{2}
\end{aligned}
$$

and

$$
K(x)=\int_{0}^{\frac{\pi}{2}} \frac{1}{\sqrt{1-x \sin ^{2}(\xi)}} d \xi \quad, \quad E(x)=\int_{0}^{\frac{\pi}{2}} \sqrt{1-x \sin ^{2}(\xi)} d \xi
$$

$K(x)$ and $E(x)$ are the elliptic integrals of the first kind and of the second kind [98]. The argument of these functions is always negative. A plot is shown in Fig. 3.11. The functions are strictly monotonic. We can use values of $K(x)$ and $E(x)$ stored in tables and interpolate, which is much faster than a numerical integration. For very small arguments we use the series expansion of $K(x)$ and $E(x)$

$$
\begin{aligned}
& K(x)=\pi\left(\frac{1}{2}+\frac{x}{8}+\frac{9 x^{2}}{128}+\frac{25 x^{3}}{512}\right)+\text { higher order terms } \\
& E(x)=\pi\left(\frac{1}{2}-\frac{x}{8}-\frac{3 x^{2}}{128}-\frac{5 x^{3}}{512}\right)+\text { higher order terms }
\end{aligned}
$$

Following our discussion in section 2.3.2, we add the field of one mirror charge that is situated at $z=2 g-z^{\prime}$, which is in our case inside the anode. The electric field of the mirror charge is obtained by simply substituting $z^{\prime}$ with $2 g-z^{\prime}$ in Eqs. 3.8a and $3.8 \mathrm{c}$. The sum of the field of the charge ring at $\left(z^{\prime}, r^{\prime}\right)$ and the mirror charge ring at $\left(2 g-z^{\prime}, r^{\prime}\right)$ gives the field of the charge that is situated at the grid point $\left(z^{\prime}, r^{\prime}\right)$. The field at $\left(r=r^{\prime}, z=z^{\prime}\right)$ is not included in the calculation of the space charge field due to the divergence of Eqs. $3.8 \mathrm{a}$ and $3.8 \mathrm{c}$ at this point. 

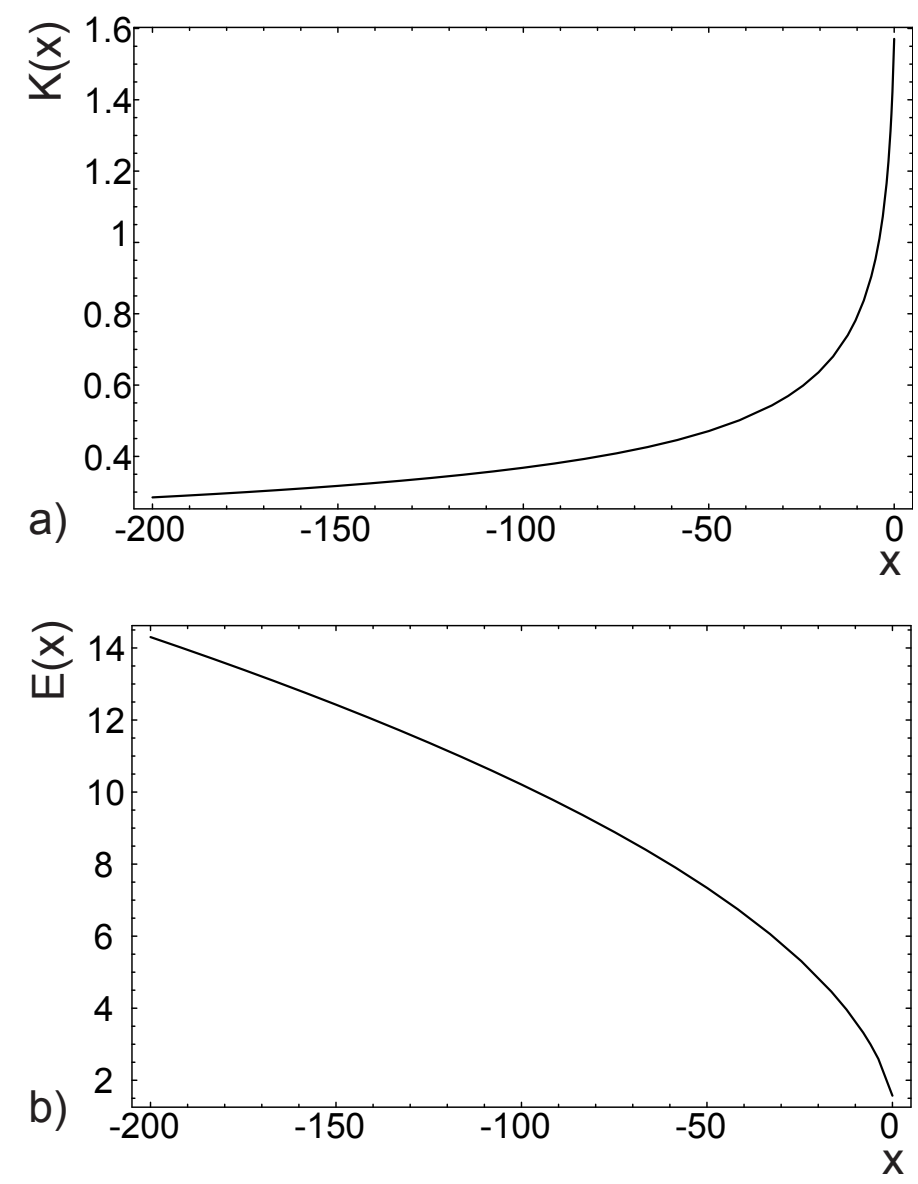

Figure 3.11: Plots of the elliptic integrals of the first kind $K(x)$ (a) and of the second kind $E(x)(\mathrm{b})$.

\subsubsection{Propagation of the Charges}

Knowing the electric field strength at each grid point, the charges can be propagated. With the absolute value of the electric field $E=|\vec{E}(r, z)|=\sqrt{E_{r}^{2}(r, z)+E_{z}^{2}(r, z)}$ at the grid point $(r, z)$, we obtain the values of

- the Townsend coefficient $\alpha(E / p)$ and attachment coefficient $\eta(E / p)$ from Fig. 2.8,

- the drift velocity $v_{D}(E / p)$ from Fig. 2.4 and

- the longitudinal and transverse diffusion coefficients $D_{L}(E / p)$ and $D_{T}(E / p)$ from Fig. 2.5.

As the next step, the electrons in each grid point are multiplied. We are using Eqs. 2.21, 2.22, 2.23 and the procedure outlined in section 3.1. The electrons are moved to a 


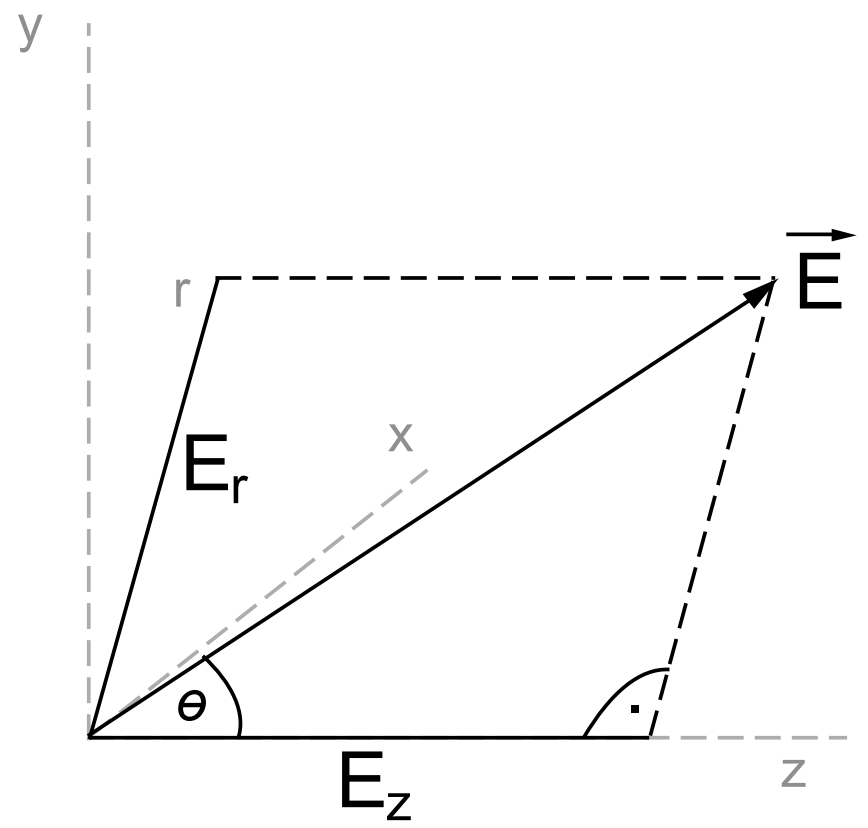

Figure 3.12: The coordinate system and the electric field vector in the two dimensional system.

new grid point that lies in the direction of the drift velocity vector $\vec{v}_{D}$, which is parallel to the vector of the electric field $\vec{E}$. We also include diffusion here, keeping in mind that longitudinal diffusion is always in the direction of $\vec{E}$, which is not necessarily parallel to the $z$-axis. Accordingly, transverse diffusion is perpendicular to $\vec{E}$. In a coordinate system with $x^{\prime}, y^{\prime}, z^{\prime}$ and with the $z^{\prime}$-axis parallel to $\vec{E}$, the propagation and diffusion are calculated the following:

- The new $x^{\prime}$-coordinate is calculated by drawing a random number from a Gaussian distribution with mean $\mu=0$ and sigma $\sigma=D_{T} \sqrt{\delta l}=D_{T} \sqrt{\left|v_{D}\right| \delta t}$. Here $\delta l$ is the drifted distance and $\delta t$ is the time step of the simulation.

- The new $y^{\prime}$-coordinate is calculated accordingly.

- The new $z^{\prime}$-coordinate is calculated by drawing a random number from a Gaussian distribution with mean $\delta l$ and sigma $\sigma=D_{L} \sqrt{\delta l}$.

Since the electric field has in the main coordinate system the direction $\theta$ (See Fig. 3.12), the propagation has to be rotated. For $\theta$ we find the relations

$$
\cos (\theta)=\frac{E_{z}}{|E|} \quad \text { and } \quad \sin (\theta)=\frac{E_{r}}{|E|}
$$


Since the problem has cylindrical symmetry we can chose $x=x^{\prime}$. The rotation is then performed by

$$
\left(\begin{array}{l}
x \\
y \\
z
\end{array}\right)=\left(\begin{array}{ccc}
1 & 0 & 0 \\
0 & \cos (\theta) & \sin (\theta) \\
0 & -\sin (\theta) & \cos (\theta)
\end{array}\right)\left(\begin{array}{l}
x^{\prime} \\
y^{\prime} \\
z^{\prime}
\end{array}\right)
$$

With the new coordinates $r=\sqrt{x^{2}+y^{2}}$ and $z$ the electrons are redistributed onto the bins. For large numbers of electrons this procedure becomes very time consuming. In that case the electrons are propagated in groups.

\subsection{The 3-D Model}

The three dimensional simulation has the following basic structure:

1. A cubic volume of the gas gap is divided into a three dimensional grid. We use Cartesian coordinates $x, y$ and $z$ (the $z$-axis is again perpendicular to the electrode plates). If the $z$-coordinate is divided into $N$ steps of size $\delta z=g / N$, the corresponding time steps of the simulation are $\delta t=\delta z / v_{D}\left(E_{0}, p\right)$, where $v_{D}\left(E_{0}, p\right)$ is the electron drift velocity from Fig. 2.4 at the applied electric field $E_{0}$.

2. One electron is put into a bin inside the volume.

3. The three dimensional electric field vector at each bin is calculated, if there is an electron in that bin.

4. The Townsend and attachment coefficients, the drift velocity and the diffusion coefficients at each bin are calculated.

5. The avalanches for each single electron are simulated using Eqs. 2.21, 2.22, 2.23 and the procedure outlined in section 3.1. Each electron is redistributed onto the bins. Here also longitudinal and transverse diffusion are included.

6. Steps 3 - 5 are repeated until all electrons left the gas gap.

The procedure is very similar to the 2-D simulation described in section 3.3. If the number of electrons in a bin exceeds a certain size, they are moved in groups. First the electric field vector $\left(E_{x}, E_{y}, E_{z}\right)$ and its norm $E$ at each bin, where electrons are situated, is calculated. To calculate the electric field of the space charge we use the potential solutions of free charges and of the mirror charges in the anode (Eqs. 2.30 to 2.32). 
The charge in the bin where the field is calculated is not included, due to the divergence of the field at this point. With the calculated field $E=|\vec{E}(x, y, z)|=\sqrt{E_{x}^{2}+E_{y}^{2}+E_{z}^{2}}$ we calculate $\alpha(E / p), \eta(E / p), D_{T}(E / p), D_{L}(E / p)$ and $v_{D}(E / p)$. The drift velocity vector $\vec{v}_{D}$ and the longitudinal diffusion are parallel to $\vec{E}$, the transverse diffusion is perpendicular to $\vec{E}$. With this information we obtain a propagation vector $\left(x^{\prime}, y^{\prime}, z^{\prime}\right)$ in the coordinate system given by $E_{x}, E_{y}, E_{z}$ :

- The new $x^{\prime}$-coordinate is calculated by drawing a random number from a Gaussian distribution with mean $\mu=0$ and sigma $\sigma=D_{T} \sqrt{\delta l}=D_{T} \sqrt{\left|v_{D}\right| \delta t}$. Here $\delta l$ is the drifted distance and $\delta t$ is the time step of the simulation.

- The new $y^{\prime}$-coordinate is calculated accordingly.

- The new $z^{\prime}$-coordinate is calculated by drawing a random number from a Gaussian distribution with mean $\delta l$ and sigma $\sigma=D_{L} \sqrt{\delta l}$.

For the values of the rotation parameters $\phi$ and $\theta$ we find the relations

$$
\tan (\phi)=\frac{E_{x}}{E_{z}} \quad \text { and } \quad \tan (\theta)=\frac{E_{y}}{\sqrt{E_{x}^{2}+E_{y}^{2}}}
$$

The propagation vector $\left(x^{\prime}, y^{\prime}, z^{\prime}\right)$ is rotated in three dimensions to give the propagation vector in the correct coordinate system $(x, y, z)$. The rotation is performed by

$$
\left(\begin{array}{l}
x \\
y \\
z
\end{array}\right)=\left(\begin{array}{ccc}
\cos (\phi) & 0 & \sin (\phi) \\
-\sin (\phi) \sin (\theta) & \cos (\theta) & \cos (\phi) \sin (\theta) \\
-\sin (\phi) \cos (\theta) & -\sin (\theta) & \cos (\phi) \cos (\theta)
\end{array}\right)\left(\begin{array}{l}
x^{\prime} \\
y^{\prime} \\
z^{\prime}
\end{array}\right)
$$

\subsubsection{Convergence of the 3-D Model}

While the accuracy of the 1-D, 1.5-D and the 2-D models was verified by increasing the number of steps and comparing the results, this approach is not practicable in the case of the 3-D model. Assuming a division of the three axes into 200 steps and that charges are situated in all bins, we must already do $\left(200^{3}\right)^{2}=6.4 \times 10^{13}$ iterations to calculate the field of all charges at all positions. Even if the field is only calculated at bins where charges are actually situated, the time needed to simulate an avalanche grows beyond reasonable values with this model. Thus, in this section we investigate the convergence behaviour expected from the 3-D model.

Generally the fields of point and line charges diverge if one approaches the charges. The fields of two- or three dimensional charge distributions do not diverge. Therefore 
we expect that the calculated space charge field will not diverge if we go to smaller step sizes. To investigate the convergence behaviour we take the electric field of some three dimensional charge distribution $\rho\left(\vec{r}^{\prime}\right)$, given by

$$
\vec{E}(\vec{r})=\frac{1}{4 \pi \varepsilon_{0}} \int_{V} \rho\left(\vec{r}^{\prime}\right) \frac{\left(\vec{r}-\vec{r}^{\prime}\right)}{\left|\vec{r}-\vec{r}^{\prime}\right|^{3}} d^{3} r^{\prime}
$$

As an example, we assume a constant charge distribution $\rho\left(\vec{r}^{\prime}\right)=1$. The absolute value of the electric field of this charge distribution is given by

$$
|\vec{E}(\vec{r})|=\frac{1}{4 \pi \varepsilon_{0}} \int_{V} \frac{1}{\left|\vec{r}-\vec{r}^{\prime}\right|^{2}} d^{3} r^{\prime}
$$

We limit the volume to a cube with side length $L$ and calculate the field at the surface at $\vec{r}=0$. The total field $E=|\vec{E}(\vec{r}=0)|$ is

$$
\begin{aligned}
E & =\frac{1}{4 \pi \varepsilon_{0}} \int_{x^{\prime}=0}^{L} \int_{y=0}^{L} \int_{z=0}^{L} \frac{1}{\left(x^{\prime}\right)^{2}+\left(y^{\prime}\right)^{2}+\left(z^{\prime}\right)^{2}} d z^{\prime} d y^{\prime} d x^{\prime} \\
& \approx \frac{1}{4 \pi \varepsilon_{0} \Delta^{2}} \sum_{i=1}^{N} \sum_{j=1}^{N} \sum_{k=1}^{N} \frac{Q_{i j k}}{i^{2}+j^{2}+k^{2}}, \quad \Delta=L / N
\end{aligned}
$$

In Eq. $3.16 b$ we introduced a discrete system where the $x-, y$ - and $z$-axes are divided into $N$ steps each. $Q_{i j k}$ is the charge in the cubicle at $\left(x^{\prime}, y^{\prime}, z^{\prime}\right)$. Since at the bin $i=j=k=0$ the field will certainly diverge, we do not include it in the calculation. If we go to smaller step sizes the charge per cubicle then decreases linearly with the volume: $Q_{i j k} \sim \Delta^{3}$. With $L=1$ we get

$$
\begin{aligned}
E & =\frac{1}{4 \pi \varepsilon_{0}} \int_{x^{\prime}=0}^{1} \int_{y=0}^{1} \int_{z=0}^{1} \frac{1}{\left(x^{\prime}\right)^{2}+\left(y^{\prime}\right)^{2}+\left(z^{\prime}\right)^{2}} d z^{\prime} d y^{\prime} d x^{\prime} \\
& \approx \frac{\Delta}{4 \pi \varepsilon_{0}} \sum_{i=1}^{N} \sum_{j=1}^{N} \sum_{k=1}^{N} \frac{1}{i^{2}+j^{2}+k^{2}}, \quad \Delta=1 / N
\end{aligned}
$$

For $N=100(1000)$ the discrete sum deviates by $7 \%$ (1\%) from the exact value. 

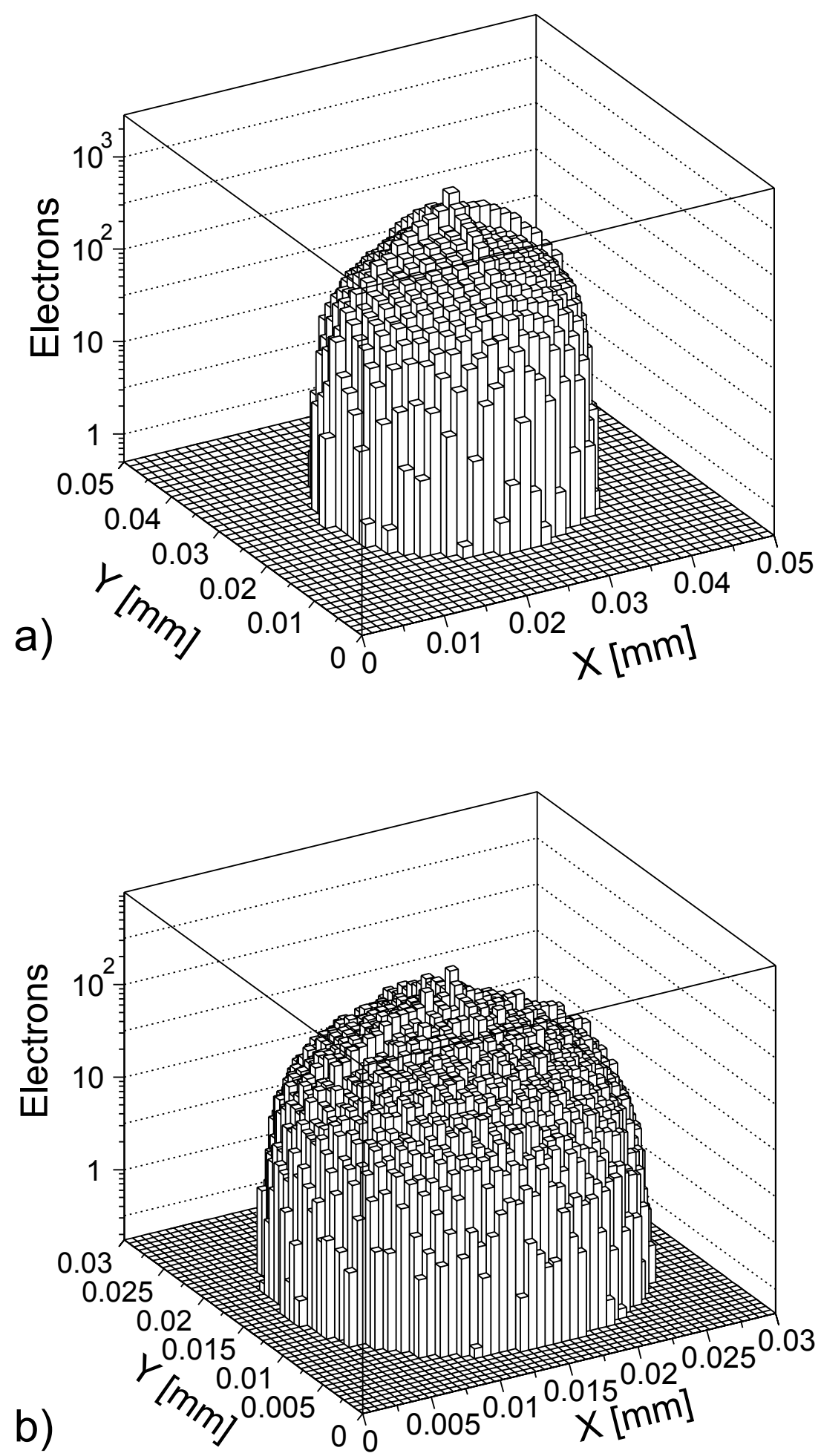

Figure 3.13: A projection on the $x y$ plane of the electron density in an avalanche simulated with the 3-D model a) We used 120 steps to divide the $x$-, $y$ - and $z$-axis. The $x$ - and $y$-axis measure from 0 to $50 \mu \mathrm{m}$. b) A similar plot for a division twice as dense in the $x$ - and $y$-direction. 


\subsubsection{Drawbacks of the 3-D Model}

Fig. 3.13 shows a projection on the $x y$ plane of the electron density in avalanches simulated with the 3-D model. We see that with the division in 120 steps in the $x$ and $y$-plane the diffusion parallel to the $x$ - and $y$-axes seems to be favored, which is of course not observed in reality. A reduction of the bin sizes leads to an improvement of the situation (Fig. 3.13b). However, as we just mentioned, computational efficiency limits the possible increase of step numbers.

A close look at Fig. 3.13 tells us that the avalanche charge distributions are to a very good approximation rotationally symmetric. As a consequence, we can certainly assume a radial symmetry of the avalanches and use the 2-D model, which does not have the same drawbacks.

\subsection{Summary}

Different simulation programs for avalanches in RPCs were described. They base on the detector physics described in chapter 2 .

In all pograms we implement the model by W. Legler for the statistics of electrons multiplication in high electric fields and at large gas gain. The gas parameters that define the primary ionization and the avalanche propagation are calculated with the programs HEED, MAGBOLTZ and IMONTE. In the 1-D model a saturation due to space charge effects is simulated by simply cutting the avalanche growth at a certain size. The program can be used for fast but detailed studies of time resolutions and efficiencies. The second model is named "1.5-D model" because here the actual avalanche propagation is simulated only in one dimension (longitudinally) but the transverse diffusion is also taken into account in the calculation of the electric field of the space charge. The 2-D model allows a very detailed simulation of both the longitudinal and the transverse avalanche development. Finally we also presented a 3-D model which turns out to be too time consuming to be used efficiently. However, since we observe a clear rotational symmetry in the avalanches, we may use the 2-D model. 


\section{Chapter 4}

\section{Geometries and Typical Operating Parameters}

In this chapter we present geometries and typical operating parameters of the RPCs we investigate in the later chapters. In our studies we will focus on the following RPC geometries:

1. The discussions of Timing RPCs in this thesis will focus on the devices that are built and tested by P. Fonte et al. (Fig. 4.1). They use gas gaps of $0.3 \mathrm{~mm}$ and resistive glass plates with $2 \mathrm{~mm}$ or $3 \mathrm{~mm}$ thickness, a volume resistivity of about $2 \times 10^{12} \Omega \mathrm{cm}$ and a relative permittivity $\varepsilon_{r}$ of 8 . The gas is $\mathrm{C}_{2} \mathrm{~F}_{4} \mathrm{H}_{2} / \mathrm{i}-\mathrm{C}_{4} \mathrm{H}_{10} /$ $\mathrm{SF}_{6}(85 \%, 5 \%, 10 \%)$. An operating voltage of $6 \mathrm{kV}(3 \mathrm{kV})$ for a double gap (single gap) results in an electric field of $100 \mathrm{kV} / \mathrm{cm}$ in the gas gaps.

2. We study Timing RPCs that use a similar design with the exception of smaller gap widths of $0.1 \mathrm{~mm}$ and $0.2 \mathrm{~mm}$.

3. We investigate Timing RPCs with gap sizes of $0.3 \mathrm{~mm}$ filled with pure isobutane.

4. Finally we investigate Trigger RPCs (Fig. 1.11) made of a $2 \mathrm{~mm}$ gas gap filled with $\mathrm{C}_{2} \mathrm{~F}_{4} \mathrm{H}_{2} / \mathrm{i}-\mathrm{C}_{4} \mathrm{H}_{10} / \mathrm{SF}_{6}(96.7 \%, 3 \%, 0.3 \%)$ and two $2 \mathrm{~mm}$ thick bakelite resistive plates with a volume resistivity around $10^{10} \Omega \mathrm{cm}$ and a relative permittivity $\varepsilon_{r}$ of 10 .

Some of the important detector and gas parameters are given in Tables 4.1 and 4.2 . For the Timing RPC shown in Fig. 4.1 b and the Trigger RPCs the weighting field in the gas gap is given by Eq. 2.40. For the single gap Timing RPCs in Fig. 4.1 a, where only one electrode is made from resistive material and the other electrode is made from metal, the weighting field is given by [I]] 

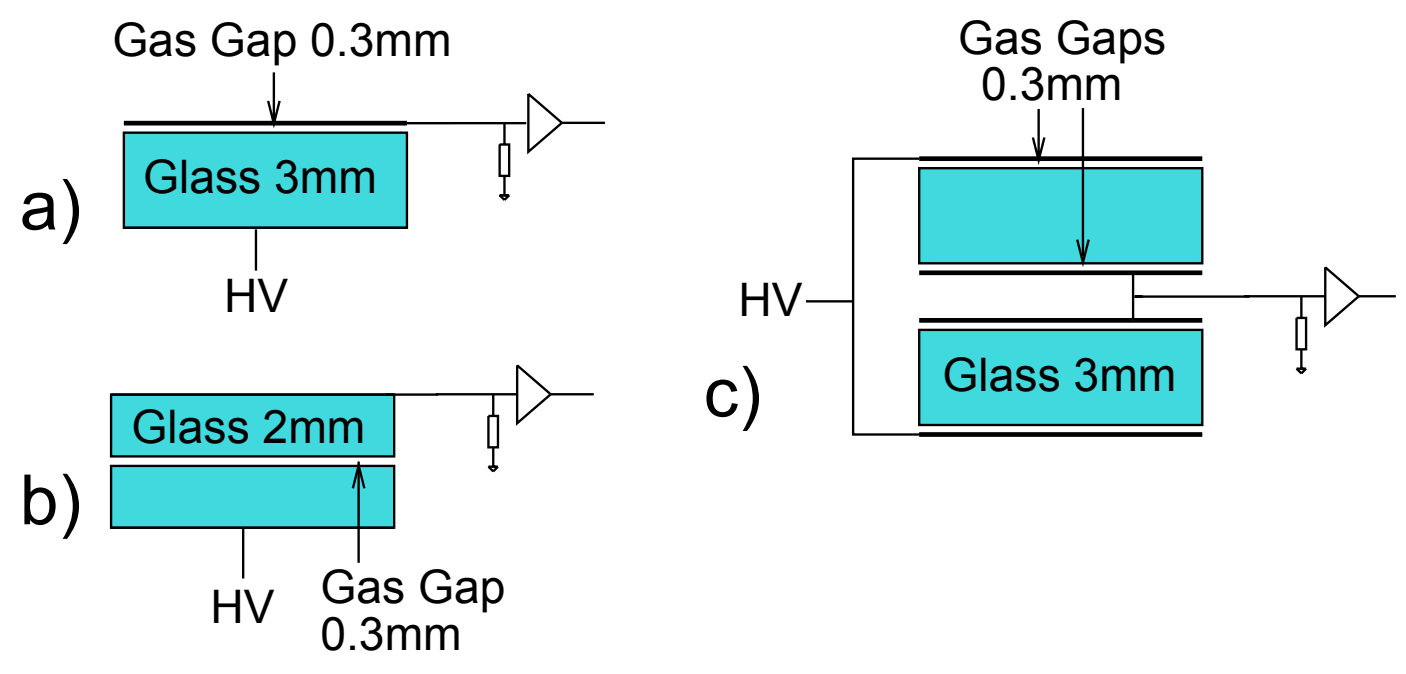

Figure 4.1: A schematic image of Timing RPCs in one and four gap configurations as in [69, 96, 97].

$$
E_{z}=\frac{\varepsilon_{r}}{q+g \varepsilon_{r}}
$$

Finally for double gaps sharing the same read out strip like in Fig. 4.1c, the weighting field is

$$
E_{z}=\frac{\varepsilon_{r}}{q+2 g \varepsilon_{r}}
$$

The 1-D model described in section 3.1 does not include the dynamic calculation of the space charge fields is used to obtain time resolution and efficiency results. Here the drift velocity $\left(v_{D}\right)$ and the Townsend and attachment coefficients $\alpha(E / p)$ and $\eta(E / p)$ are set constant. We use the values at the applied electric field strength and take them from Figs. 2.4 and 2.8. The cluster size distributions for the primary ionization are taken from Fig. 2.3. The number of electrons at which the avalanches are saturating is $N_{\text {sat }}=1.6 \times 10^{7}$ for the Timing RPC and $N_{\text {sat }}=5 \times 10^{7}$ electrons for the Trigger RPC.

The 1.5-D model also includes diffusion and space charge effects. It is described in section 3.2 and is used study in more detail the space charge effect and to collect charge spectra. We use the dependencies of the drift velocity $\left(v_{D}(E / p)\right)$ and of the Townsend and attachment coefficients $(\alpha(E / p)$ and $\eta(E / p))$ on the electric field strength from Figs. 2.4and 2.8). The diffusion coefficients $\left(D_{L}(E / p)\right.$ and $\left.D_{T}(E / p)\right)$ are set constant. We use the values at the applied electric field strength $\left(E_{0}=5 \mathrm{kV} / \mathrm{mm}\right.$ for the Trigger RPC and $E_{0}=10 \mathrm{kV} / \mathrm{mm}$ for the Timing RPC). 


\begin{tabular}{|c|c||c|c|c|c|c|c|c|}
\hline RPC & gas & $q[\mathrm{~mm}]$ & $g[\mathrm{~mm}]$ & $\varepsilon_{r}$ & $E_{W}[/ \mathrm{mm}]$ & $\lambda[\mathrm{mm}]$ & $n_{a v}$ & $Q_{t}[\mathrm{pC}]$ \\
\hline \hline Timing & $85 / 5 / 10$ & 2 & 0.3 & 8 & 1.25 & 0.11 & 2.6 & 0.02 \\
\hline Timing & $85 / 5 / 10$ & 2 & 0.1 & 8 & 1.67 & 0.11 & 2.6 & 0.02 \\
\hline Timing & $85 / 5 / 10$ & 2 & 0.2 & 8 & 1.43 & 0.11 & 2.6 & 0.02 \\
\hline Timing & $\mathrm{i}^{-} \mathrm{C}_{4} \mathrm{H}_{10}$ & 2 & 0.3 & 8 & 1.25 & 0.11 & 1.9 & 0.02 \\
\hline Trigger & $96.7 / 3 / 0.3$ & 2 & 2 & 10 & 0.417 & 0.104 & 2.8 & 0.1 \\
\hline
\end{tabular}

Table 4.1: Simulation parameters for the diffent RPCs. $q$ is the thickness of the resistive layers, $g$ is the gap size, $\varepsilon_{r}$ is the relative permittivity of the resistive material, $E_{W}$ is value of the $z$-component of the weighting field, $\lambda$ is the mean free path for the primary ionization, $n_{a v}$ is the average number of electrons per cluster and $Q_{t}$ is the charge threshold. We assume the geometry shown in Figs. 4.1 b and 1.11 and calculate the weighting field from Eq. 2.40. For the geometries in Figs. 4.1 a and 4.1c $E_{W}$ will have different values (Eqs. 4.1 and 4.2). As ionizing particles we assume $120 \mathrm{GeV}$ muons for the Trigger RPC and $7 \mathrm{GeV}$ pions for the Timing RPCs.

\begin{tabular}{|c|c||c|c|c|c|c|}
\hline RPC & gas & $\mathrm{HV}[\mathrm{kV}]$ & $\alpha[1 / \mathrm{mm}]$ & $\eta[1 / \mathrm{mm}]$ & $D_{L}[\sqrt{\mathrm{mm}}]$ & $D_{T}[\sqrt{\mathrm{mm}}]$ \\
\hline \hline Timing & $85 / 5 / 10$ & 3.0 & 123 & 10.5 & 0.033 & 0.027 \\
\hline Timing & $85 / 5 / 10$ & 1.6 & 278 & 5.5 & 0.033 & 0.027 \\
\hline Timing & $85 / 5 / 10$ & 2.1 & 135 & 10 & 0.033 & 0.027 \\
\hline Timing & $\mathrm{i}-\mathrm{C}_{4} \mathrm{H}_{10}$ & 2.8 & 87 & 0 & 0.028 & 0.023 \\
\hline Trigger & $96.7 / 3 / 0.3$ & 10.0 & 13.3 & 3.5 & 0.027 & 0.032 \\
\hline
\end{tabular}

Table 4.2: Simulation parameters for different RPCs. We list typical values for the high voltage, for the Townsend and attachment coefficients $\alpha(E / p)$ and $\eta(E / p)$ and for the diffusion coefficients $D_{L}(E / p)$ and $D_{T}(E / p)$.

\section{Expected values}

Analytic formulas for efficiency, time resolution and average charges of single gap RPCs are derived in [1]]. We list the formulas here and give typical expected values in Table 4.3. The intrinsic time resolution of the detector (no electronics) is given by

$$
\sigma_{t}=\frac{1.28}{(\alpha-\eta) v_{D}}
$$

In the derivation of Eq. 4.3 it is assumed that no space charge effects are present, which means that $(\alpha-\eta)$ and $v_{D}$ are constant at all times. We find the interesting result that the time resolution does not depend on the threshold, which is also observed in measurements [97]. For the efficiency of single gap RPCs we find 


\begin{tabular}{|c|c||c|c|c|c|c|c|}
\hline RPC & gas & $g[\mathrm{~mm}]$ & $\mathrm{HV}[\mathrm{kV}]$ & $\epsilon[\%]$ & $\sigma_{t}[\mathrm{ps}]$ & $\left\langle Q_{\text {tot }}\right\rangle[\mathrm{pC}]$ & $\left\langle Q_{\text {ind }}\right\rangle[\mathrm{pC}]$ \\
\hline \hline Timing & $85 / 5 / 10$ & 0.3 & 3.0 & 76 & 54 & $1.8 \times 10^{7}$ & $1.9 \times 10^{5}$ \\
\hline Timing & $85 / 5 / 10$ & 0.1 & 1.6 & 31 & 16 & $1.1 \times 10^{4}$ & 64 \\
\hline Timing & $85 / 5 / 10$ & 0.2 & 2.1 & 48 & 47 & $2.6 \times 10^{3}$ & 27 \\
\hline Timing & ${\mathrm{i}-\mathrm{C}_{4} \mathrm{H}_{10}}$ & 0.3 & 2.8 & 74 & 117 & $7.6 \times 10^{3}$ & 110 \\
\hline Trigger & $96.7 / 3 / 0.3$ & 2 & 10 & 90 & 950 & 200 & 6 \\
\hline
\end{tabular}

Table 4.3: Expected values for the efficiency $(\epsilon)$, time resolution $\left(\sigma_{t}\right)$, the mean total signal charge $\left(\left\langle Q_{t o t}\right\rangle\right)$ and the mean induced charge $\left(\left\langle Q_{i n d}\right\rangle\right)$ for different detector types. $g$ is the gap width.

$$
\epsilon=1-e^{-\left(1-\frac{\eta}{\alpha}\right) \frac{g}{\lambda}}\left[1+\frac{\alpha-\eta}{E_{w} e_{0}} Q_{t}\right]^{\frac{1}{\alpha \lambda}}
$$

where $e_{0}$ is the unit charge and $Q_{t}$ is the charge threshold. The efficiency depends explicitly on $\alpha$ and $\eta$ and not only on the effective Townsend coefficient. For $\alpha \rightarrow \infty$ or $Q_{t} \rightarrow 0$ the maximum detection efficiency becomes $1-\exp (-d / \lambda)$, as in Eq. 2.6. $\exp (-d / \lambda)$ is the probability to find no cluster in the gas gap. For larger gap sizes the formula underestimates the efficiency since it does not take into account the case where individual clusters stay below the threshold, while the sum of them crosses the threshold.

The total signal charge $Q_{t o t}$ is the charge of all positive ions $N^{+}$in the gap at the end of the signal development when all electrons have either left the gap or got attached. The expected average total signal charge $\left\langle Q_{t o t}\right\rangle$ is thus given by the average number of positive ions $\left\langle N^{+}\right\rangle$as

$$
\begin{aligned}
\left\langle Q_{t o t}\right\rangle=e\left\langle N^{+}\right\rangle & =\frac{e n_{a v} \alpha}{\alpha-\eta}\left[\frac{1}{\lambda(\alpha-\eta)}\left(e^{(\alpha-\eta) g}-1\right)-\frac{g}{\lambda}\right] \\
& \approx \frac{e n_{a v} \alpha}{\lambda(\alpha-\eta)^{2}} e^{(\alpha-\eta) g} \quad \text { for } \quad e^{(\alpha-\eta) g} \gg 1 .
\end{aligned}
$$

The induced charge (or fast charge) $Q_{\text {ind }}$ is the charge that is induced on the read out strip by the propagation of the electrons in the gas gap of the RPC. The average induced charge $\left\langle Q_{i n d}\right\rangle$ is proportional to the number of ions and is calculated as

$$
\left\langle Q_{i n d}\right\rangle=\frac{E_{W}}{\alpha}\left\langle Q_{t o t}\right\rangle \approx \frac{E_{W} n_{a v} e}{\lambda(\alpha-\eta)^{2}} e^{(\alpha-\eta) g} .
$$


The values shown in Table 4.3 are reasonable with the exception of the average charges. Especially in the case of the $0.3 \mathrm{~mm}$ gap Timing RPC a total signal charge of $1.8 \times 10^{7} \mathrm{pC}$ is in vast contradiction with the measured values of around $5 \mathrm{pC}$.

Finally we also want to investigate the shape of the charge spectra we expect with the given avalanche statistics, using the example of a single gap Timing RPC. We assume that we have one electron in the gas gap. Its position is exponentially distributed and given by Eq. 2.1

$$
P_{1}(z)=\frac{1}{\lambda} \exp \left(-\frac{z}{\lambda}\right)
$$

Moreover, we assume that the attachment coefficient is zero and that the Townsend coefficient is constant, which is the same as neglecting the presence of a space charge effect. For random avalanches the avalanche multiplication over the distance $g-z$ follows Eqs. 2.14, which in our case become

$$
\begin{aligned}
& P_{2}(n=0, g-z)=0 \\
& P_{2}(n>0, g-z)=\frac{1}{\bar{n}(g-z)}\left(1-\frac{1}{\bar{n}(g-z)}\right)^{n-1}
\end{aligned}
$$

For a distance $g-z$, where $n$ is sufficiently large, we can approximate Eq. $4.8 b$ by

$$
P_{2}(n, g-z)=\frac{1}{\bar{n}(g-z)} \exp \left(-\frac{n}{\bar{n}(g-z)}\right)
$$

Both the starting position $z$ of the avalanche and the avalanche charge at a distance $g-z$ are exponentially distributed. The probability to find $n$ electrons at $g$ in a random avalanche that started at the random position $z$ with Townsend coefficient $\alpha$ is

$$
\begin{aligned}
P(n, g, \alpha) & =\int_{0}^{g} P_{1}(z) P_{2}(n, g-z) d z \\
& =\frac{1}{\lambda} \int_{0}^{g} \exp \left(-\frac{z}{\lambda}-\alpha(g-z)-\frac{n}{\exp (\alpha(g-z))}\right) d z .
\end{aligned}
$$

Eq. $4.10 b$ can only be evaluated numerically. Fig. 4 shows plots for typical Timing RPC values. Fig. $4 \mathrm{~b}$ shows that the function is linear on a double-logarithmic plot, which indicates that the spectrum follows a power law for the given specific parameters. 

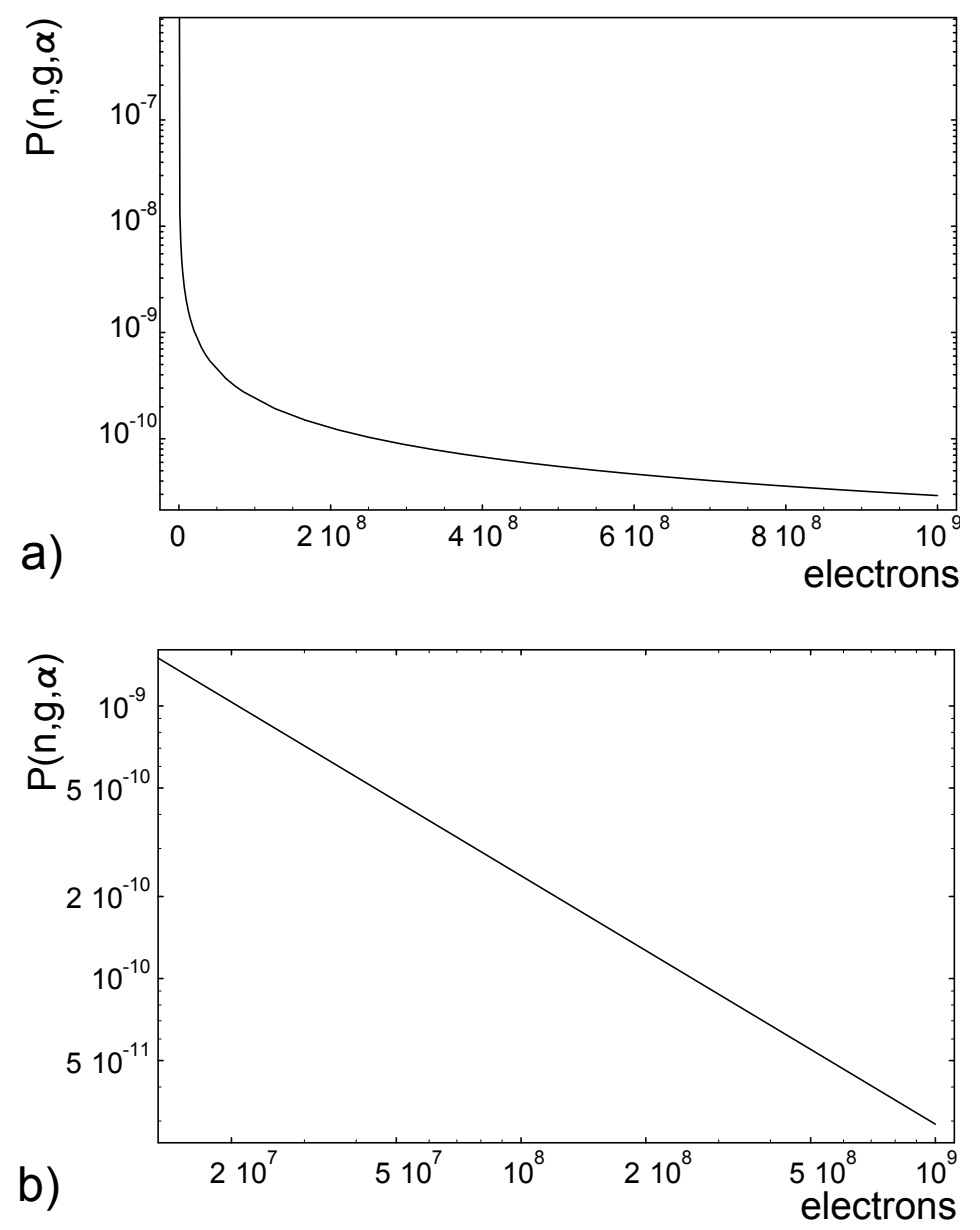

Figure 4.2: a) A plot of Eq. $4.10 \mathrm{~b}$ for $g=0.3 \mathrm{~mm}, \lambda=0.1 \mathrm{~mm}$ and $\alpha=113 / \mathrm{mm}$. b) The same plot as in a) on a double-logarithmic scale. 


\section{Chapter 5}

\section{Results Obtained with the 1-D Model}

In this chapter we present results on the simulation of Resistive Plate Chambers that were obtained with the one dimensional simulation model (1-D model) described in section 3.1. The results presented here were published in [1]].

\subsection{Efficiency and Time Resolution}

Fig. 5.1 shows the efficiency and time resolution of single and quad gap Timing RPCs versus voltage. The single gap Timing RPC was simulated for the geometry from Fig. 4.1b with the gas $\mathrm{C}_{2} \mathrm{~F}_{4} \mathrm{H}_{2} / \mathrm{i}-\mathrm{C}_{4} \mathrm{H}_{10} / \mathrm{SF}_{6}(85 \%, 5 \%, 10 \%)$ at 970 mbar. We use a weighting field of $1.25 / \mathrm{mm}$. With $7 \mathrm{GeV}$ pions we find 9.13 clusters per mm (Fig. 2.2). The threshold is $20 \mathrm{fC}$. The amplifier peaking time is $200 \mathrm{ps}$, the noise $1 \mathrm{fC}$ and the Townsend and attachment coefficient and drift velocity are chosen at the applied electric field from Figs. 2.4 and 2.8. The simulation results shown in Fig. 5.1 a reproduce quite well the measured data from [99]. We obtain efficiencies of around $75 \%$ and a time resolution around $50 \mathrm{ps}$. The formulas for the time resolution and efficiency from Eqs. 4.3 and 4.4 are also overlayed. The values are close to the results from the Monte-Carlo simulations.

The simulation for the quad gap RPC was done for the geometry from Fig. 4.11c. Here we use similar parameters as for the single gap Timing RPC except for the weighting field from Eq. 4.2, giving $1.026 / \mathrm{mm}$, and an amplifier peaking time of $3 \mathrm{~ns}$. The simulation results are shown in Fig. 5.1 b. Again we find good agreement of simulated efficiencies and time resolution with the measurements from [99].

Simulated efficiencies and time resolution for a single gap Trigger RPC as in Fig. 1.11 are shown in Fig. 5.2. The gas mixture is $\mathrm{C}_{2} \mathrm{~F}_{4} \mathrm{H}_{2} / \mathrm{i}-\mathrm{C}_{4} \mathrm{H}_{10} / \mathrm{SF}_{6}(96.7 \%, 3 \%$, $0.3 \%$ ). For $\varepsilon_{r}=10$ the weighting field from Eq. 2.40 gives $0.417 / \mathrm{mm}$. A $120 \mathrm{GeV}$ muon leaves 9.64 clusters per $\mathrm{mm}$. We assume a preamplifier peaking time of $1.3 \mathrm{~ns}$. The induced charge is divided by two, accounting for the termination of the RPC strips 

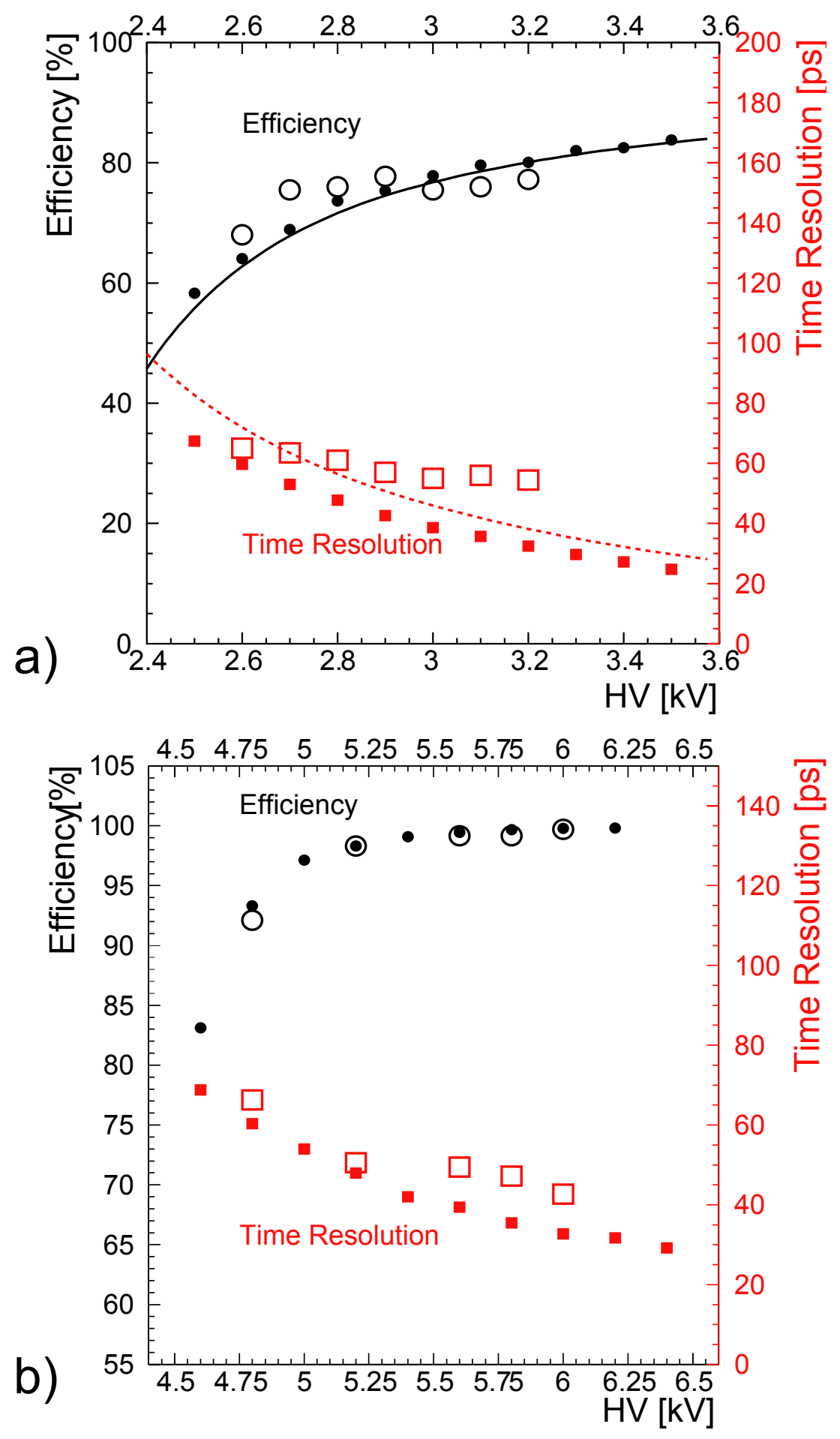

Figure 5.1: The simulated efficiencies and time resolution [1] of single gap (a) and quad gap (b) Timing RPCs as in Fig. 4.1b and 4.1c for the parameters mentioned in the text, temperature $T=296.15 \mathrm{~K}$ and pressure $p=970 \mathrm{mbar}$. The open symbols are measurements from [99]. For the single gap RPC the formulas for the time resolution and efficiency from Eqs. 4.3 and 4.4 are overlayed. 

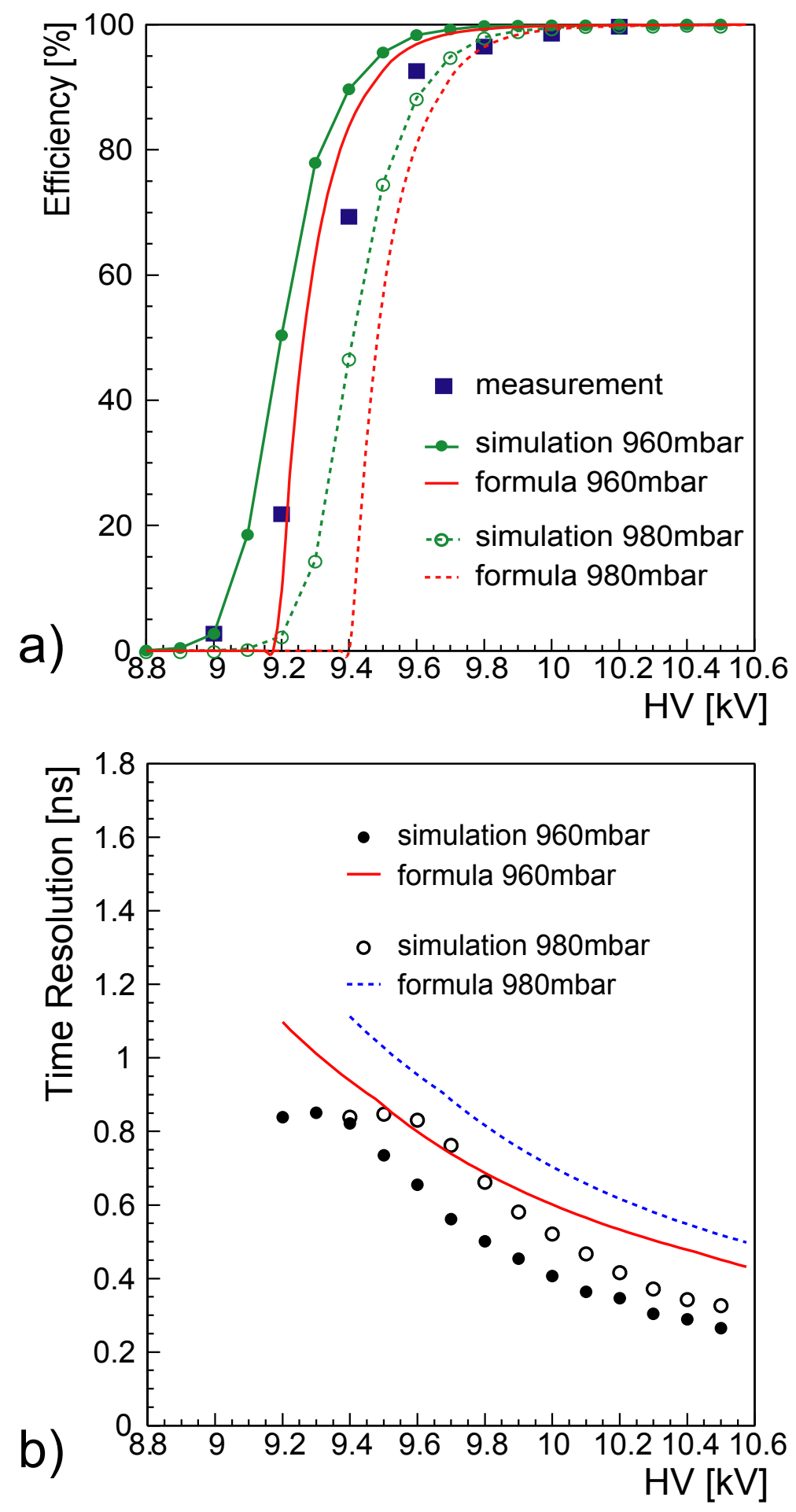

Figure 5.2: The simulated efficiencies (a) and time resolution (b) of a single gap Trigger RPCs with a geometry as in Fig. [1.11. We also show measurements from [100]. The temperature and pressure is $T=296.15 \mathrm{~K}$ and $p=960 \mathrm{mbar}, 980 \mathrm{mbar}$ [1]]. 


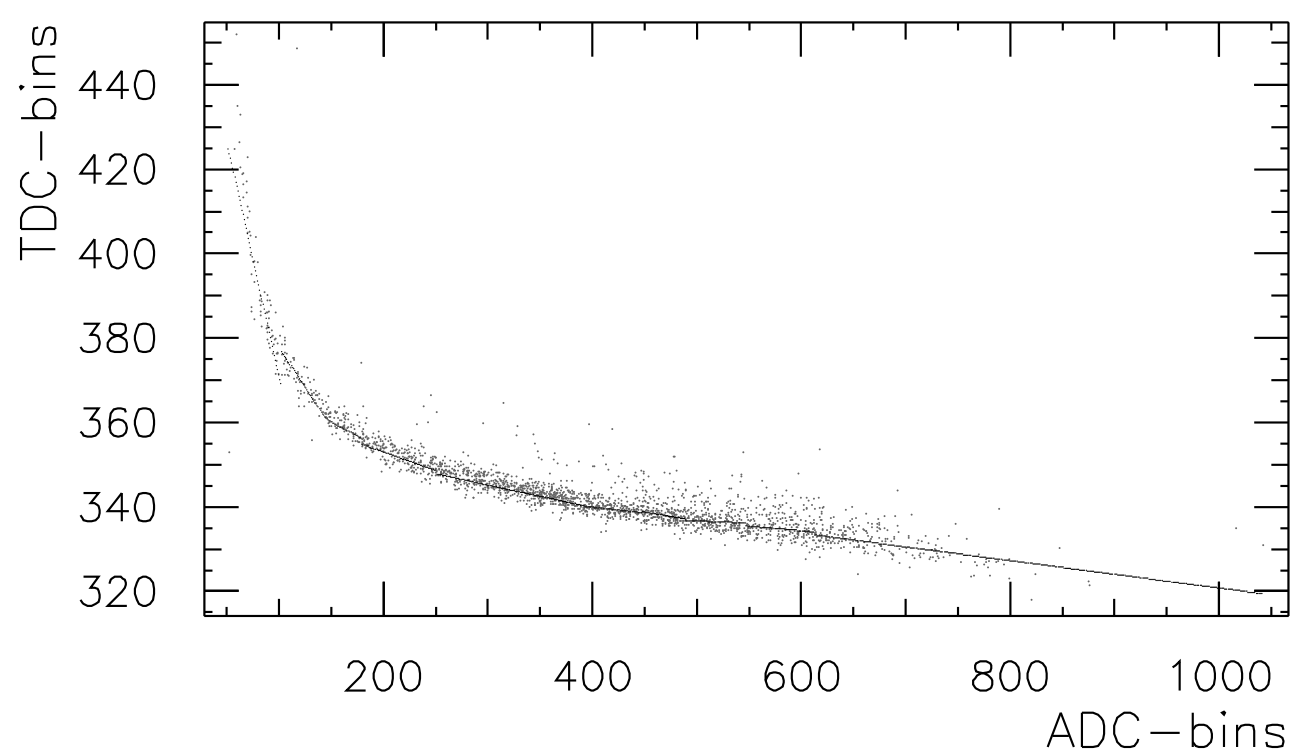

Figure 5.3: A measured charge-to-time correlation for a quad gap RPC together with a correction curve [97].

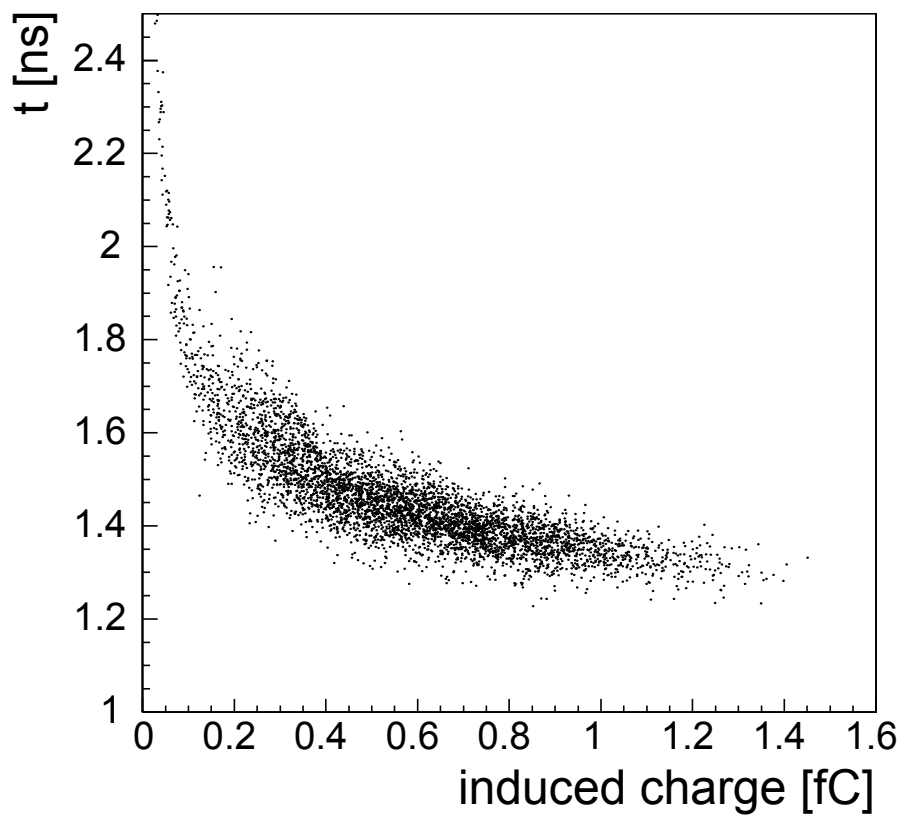

Figure 5.4: A charge to time correlation simulated with the simple 1-D model described in section 3.1 for a quad gap RPC at $5200 \mathrm{~V}$ [11]. 
and a $100 \mathrm{fC}$ threshold is applied. The measurements from [100] are quite well reproduced by the simulation.

\subsection{Charge-to-Time Correlation}

The correlation of the threshold crossing time of the signals in Timing RPCs to the induced charge is commonly used to improve the time resolution of the device. As an example we show a measurement from [97] for the quad gap Timing RPC from 4.1c (Fig. 5.3). The fit to the data, which is used as the correction curve, is also shown. Fig. 5.4 shows the simulated charge-to-time correlation for a similar quad gap RPC. We used the same parameters as given in section 5.1. The agreement between the simulation and the measurement is quite acceptable.

The charge-to-time correlation is caused by fundamental detector effects as well as the amplifier electronics. The finite rise time of the amplifier introduces an additional time jitter through the pulse height fluctuations of the signal. The intrinsic detector charge-to-time correlation is the topic in section 6.4.

\subsection{Summary}

The Monte-Carlo-Simulation of efficiencies and time resolution of single and quad gap Timing RPCs and single gap Trigger RPCs gives results that are close to measurements. The high efficiency of single gap Timing RPCs with $0.3 \mathrm{~mm}$ gas gaps is explained by the large ionization density of around 9.5 clusters per $\mathrm{mm}$ and the large effective Townsend coefficient of around $113 / \mathrm{mm}$. 


\section{Chapter 6}

\section{Results Obtained with the 1.5-D Model}

In this chapter we present results on the simulation of Resistive Plate Chambers that were obtained with the 1.5-D model described in section 3.2. We start with an investigation of the influence of the space charge effect on the signal rise time (6.1). In section 6.2 we present simulated charge spectra and compare them to measurements. In section 6.3 we investigate the operational mode of RPCs, using the example of the Timing RPC. We shall see that it is strongly influenced by space charge effects. In section 6.4 we present detector-intrinsic charge-to-time correlations and in section 6.5 we show results on simulated avalanches in Timing RPCs filled with pure isobutane. Finally we discuss streamers in section 6.6.

\subsection{Signal Rise Time}

At the threshold level of 10 to $100 \mathrm{fC}$ the avalanche in an RPC generally consists of more than $10^{6}$ electrons. It turns out that at that level the space charge effect does already have an influence on the avalanche growth and hence the signal rise time. Figs. 6.1 b and Fig. 6.2b show the intrinsic time resolutions for single gap Timing RPCs at $3 \mathrm{kV}$ and for single gap Trigger RPCs at $10 \mathrm{kV}$. For the Timing RPC and for the Trigger RPC we show the distributions for two cases: In the first case we included the space charge effect in the simulation, in the second we did not include it. We find that the mean is shifted slightly but the root mean squared (r.m.s.) is amost unaffected. It seems that even though the space charge effect affects the signal rise time, its influence on the time resolution is negligible. To illustrate this result, we investigate single avalanches in Fig. 6.3, where the induced charge versus time is plotted for ten avalanches that were started by single electrons. As we have mentioned in section 3.1, the very beginning of the avalanche determines its final size. This means that the fluctuation in the threshold crossing time, which determines the time resolution of an RPC, is caused by the avalanche fluctuations at a level where the avalanche is still small. An avalanche that is initially growing more rapidly reaches the threshold 


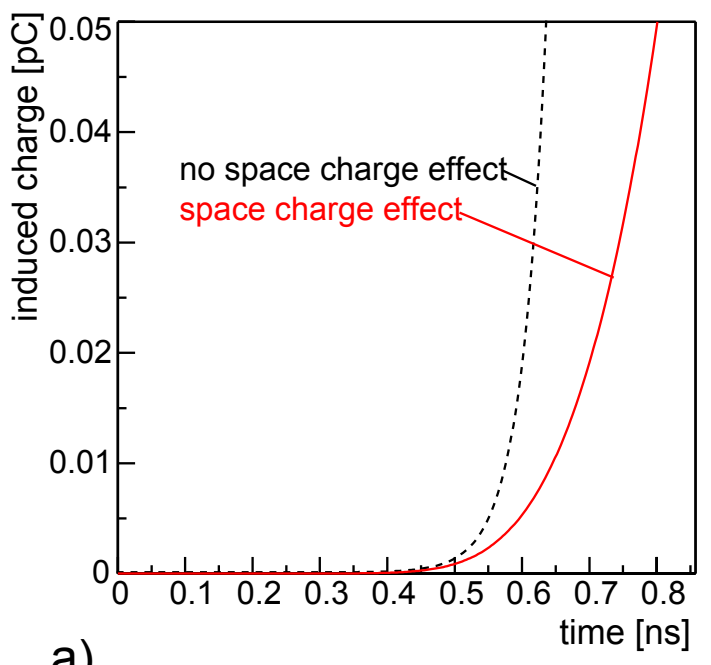

a)

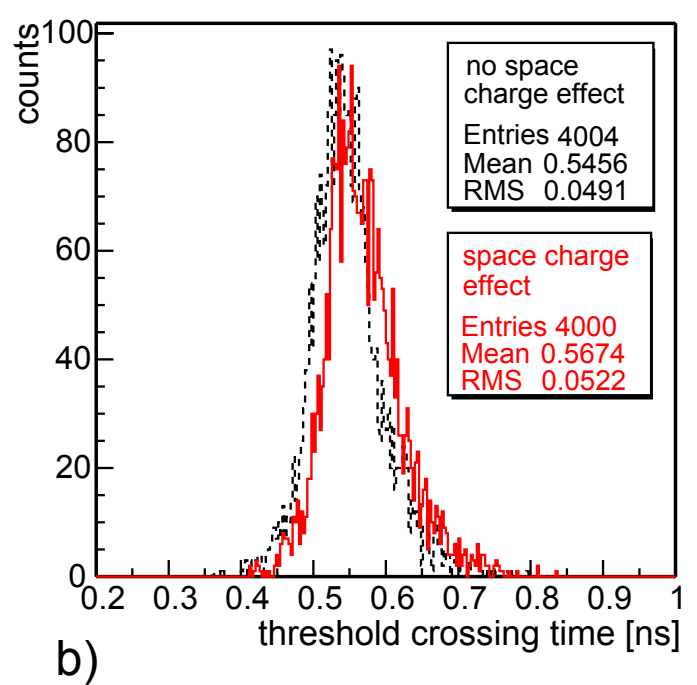

b)

Figure 6.1: The influence of the space charge effect on the time resolution of Timing RPCs. We simulated a single gap Timing RPC at $3 \mathrm{kV}$, temperature $T=296.15 \mathrm{~K}$ and pressure $p=970$ mbar. a) A Comparison of simulated average signals at an early stage. We find that at the threshold level (typically $20 \mathrm{fC}$ ) the space charge effect does already influence the signal rise. b) Simulated intrinsic time resolutions (no amplifier). The time resolution is almost unaffected while the mean of the distribution is shifted slightly by the space charge effect.

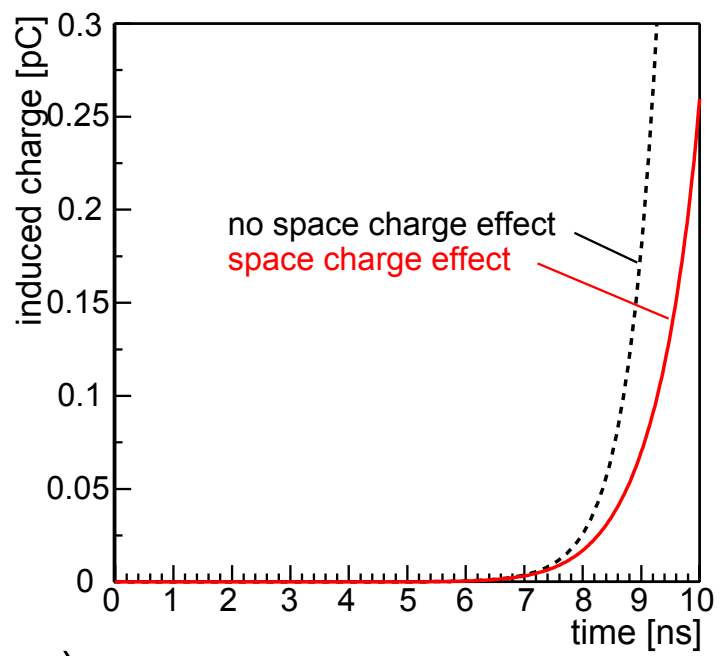

a)

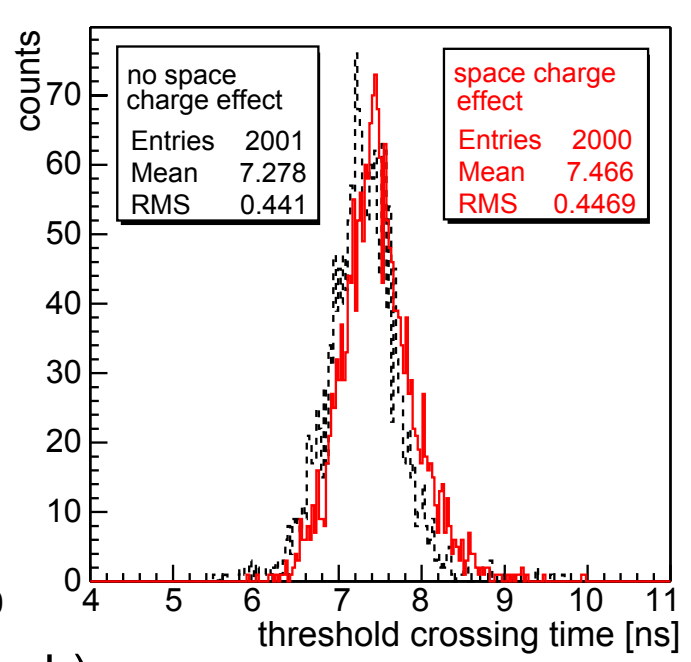

b)

Figure 6.2: The influence of the space charge effect on the time resolution of Trigger RPCs. We simulated a single gap Trigger RPC at $10 \mathrm{kV}$, temperature $T=296.15 \mathrm{~K}$ and pressure $p=970$ mbar. a) A Comparison of simulated average signals at an early stage. At the threshold level (typically $100 \mathrm{fC}$ ) there is already an influence of the space charge on the signal rise. b) Simulated intrinsic time resolutions (no amplifier). Again, the time resolution is almost unaffected while the mean of the distribution is shifted slightly by the space charge effect. 


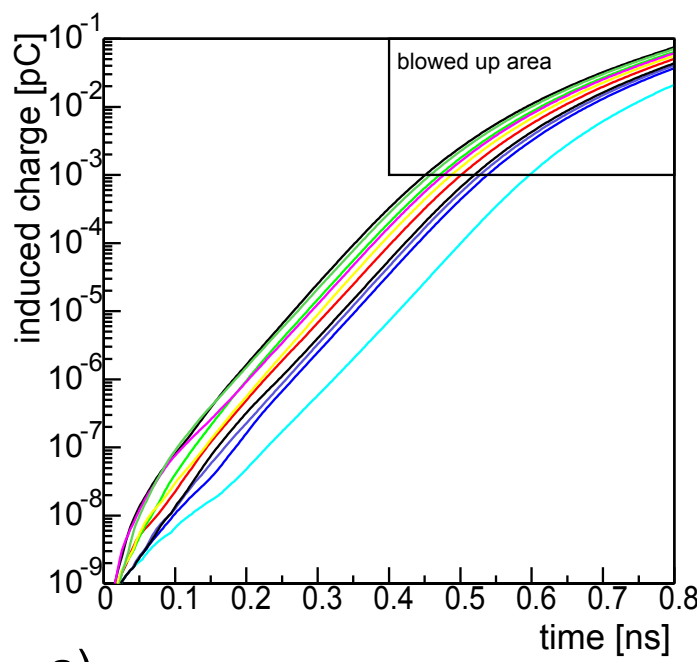

a)

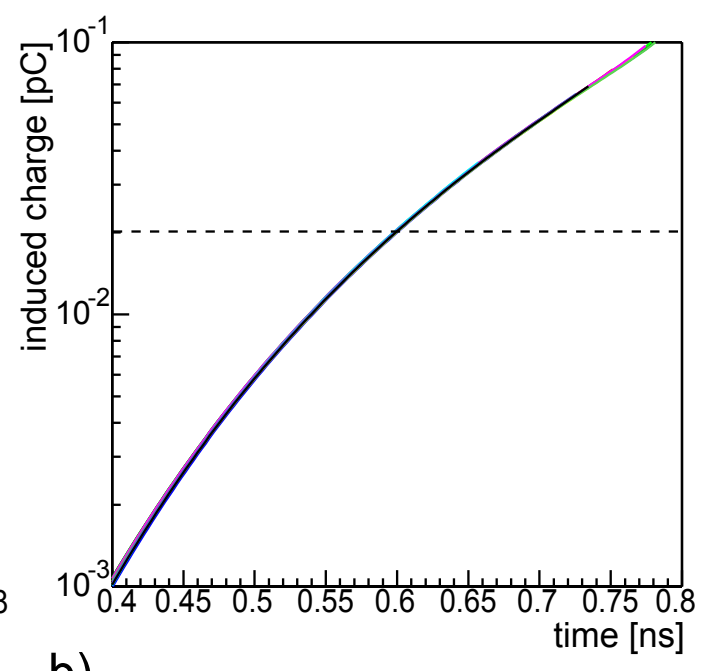

b)

Figure 6.3: a) Some simulated avalanches in Timing RPCs. All avalanches were started by one electron in the same step. The fluctuation in the induced charge is large in the beginning when there are just a few electrons. At all later stages the avalanches grow similarly. b) A blow up of the indicated region above the threshold level of $10 \mathrm{fC}$. All 10 curves are shifted along the time axis so that they match at a threshold of $20 \mathrm{fC}$. We find that the shapes of the curves match very nicely.

sooner than an avalanche that undergoes a slow initial growth. Once the avalanche has reached a sufficient size ( $\gtrsim 10 \mathrm{fC})$, it grows exponentially like $e^{(\alpha-\eta) z}$, if we neglect a space charge effect. Thus the avalanche growth is similar for different avalanches, once they have reached that size. The space charge effect introduces a deviation in the signal rise from an exponential (saturation), which explains the shift of the mean times. However, this deviation is similar for all signals so that the time resolution is not affected. If we overlay the different curves from Fig. 6.3 a at the threshold level, which is done in Fig. 6.3 b, we find that they are almost indistinguishable.

Finally we want to compare the signal rise times to measurements. An induced current signal would rise like $\exp \left(f_{0} t\right)$, where $f_{0}=(\alpha-\eta) v_{D}$, if no space charge effect is present. In [101] the authors show that sending this signal through a general linear network, the output signal shows the same exponential rise and $f_{0}$ can be measured by setting two thresholds $Q_{1}$ and $Q_{2}$ to the signal. From the two threshold crossing times $t_{1}$ and $t_{2}$ one finds $f_{0}$ by

$$
\ln \left(Q_{2} / Q_{1}\right)=f_{0}\left(t_{2}-t_{1}\right)
$$

This relation holds only if the input signal is exponential at the threshold crossing times. Fig. 6.4 shows measured [102] and simulated values of $f_{0}$ for different voltages 


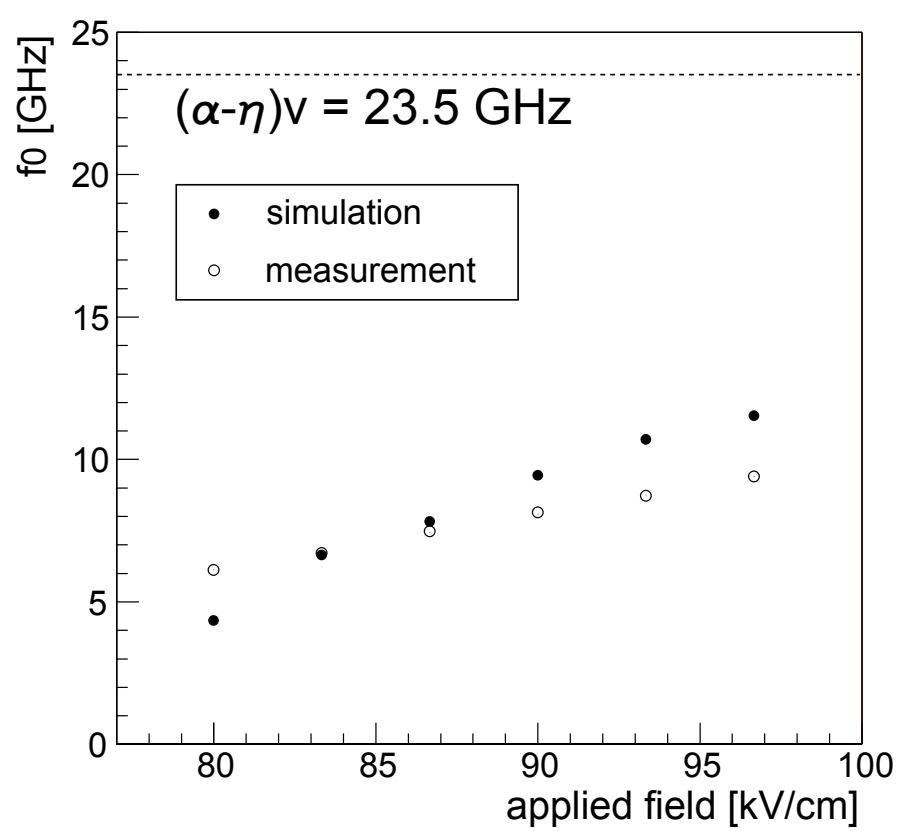

Figure 6.4: The value of $f_{0}=(\alpha-\eta) v_{D}$. We show simulated values (the solid circles) for $Q_{1}=40 \mathrm{fC}$ and $Q_{2}=80 \mathrm{fC}$ and measurements (open circles) from [102].

in single gap Timing RPCs. The simulation is quite close to the measurement from. The deviation of the measured and the simulated value of the expected value $f_{0}=(\alpha-$ 7) $v_{D}=23.5 \mathrm{GHz}$ is the consequence of the non-exponential growth of the avalanches at the threshold level due to the space charge effect, as it was plotted in Figs. 6.1 and Fig. 6.2.

\subsection{Charge Spectra}

In this section we present spectra of the induced and the total signal charge:

The induced charge or fast charge $Q_{i n d}$ is the charge that is induced on the read out strip by the moving electrons.

The total signal charge $Q_{t o t}$ is the charge of all positive ions in the gap at the end of the signal when all electrons have either left the gap or got attached.

We chose 500 steps for the 1.5-D simulation of avalanches and accumulated charge spectra of the induced charge $Q_{i n d}$ and of the signal charge $Q_{t o t}$ for Timing and for Trigger RPCs. The average charges obtained with the Monte-Carlo simulation without a space charge effect are: 


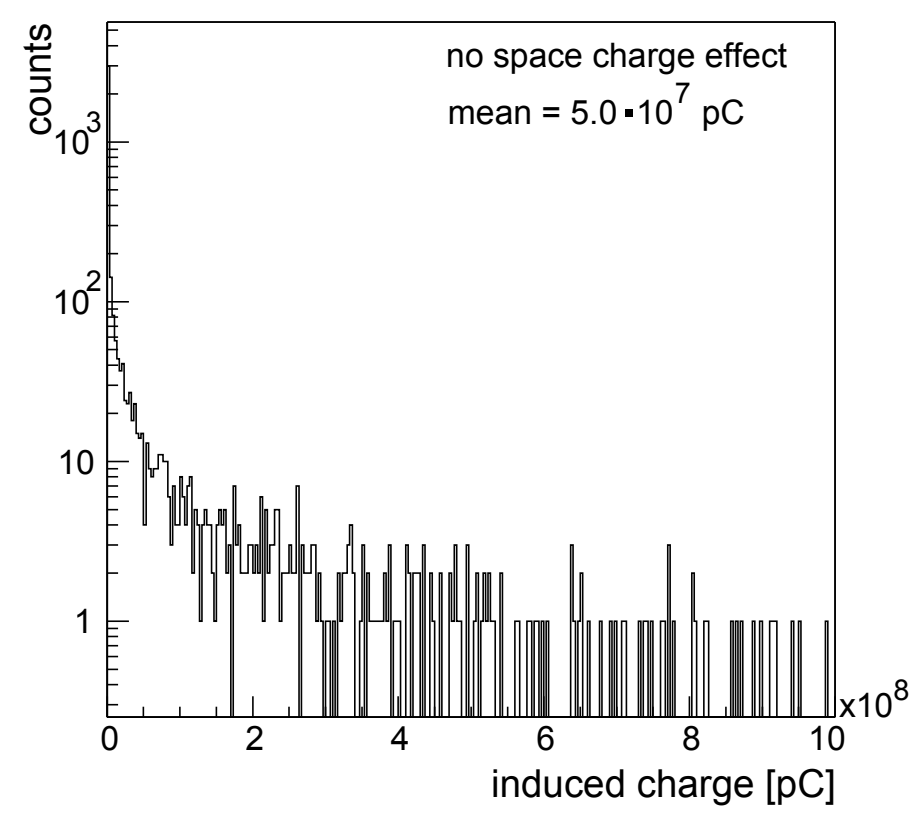

Figure 6.5: A simulated Timing RPC charge spectrum without space charge effect at $3 \mathrm{kV}$, temperature $T=296.15 \mathrm{~K}$ and pressure $p=970 \mathrm{mbar}$.

$$
\begin{array}{lll}
\text { Timing RPC: } & Q_{\text {ind }} \approx 5 \times 10^{7} \mathrm{pC}, & Q_{\text {tot }} \approx 5 \times 10^{9} \mathrm{pC}, \\
\text { Trigger RPC: } & Q_{\text {ind }} \approx 7.9 \times 10^{3} \mathrm{pC}, & Q_{\text {tot }} \approx 2.6 \times 10^{5} .
\end{array}
$$

The values are even larger than the ones obtained with the analytic formulas (Eqs. 4.5 and 4.6). This is due to the fact that in the simulations we also included diffusion. As was shown in section 3.2.1, longitudinal diffusion generally increases the avalanche charge. In experiment one measures values that are several orders of magnitudes smaller [69, 96, 97, 39]:

$$
\begin{array}{lll}
\text { Timing RPC: } & Q_{\text {ind }} \approx 0.3 \mathrm{pC}, & Q_{\text {tot }} \approx 5 \mathrm{pC}, \\
\text { Trigger RPC: } & Q_{\text {ind }} \approx 2 \mathrm{pC}, & Q_{\text {tot }} \approx 40 \mathrm{pC} .
\end{array}
$$

We observe a discrepancy of up to nine orders of magnitude! Only a very strong space charge effect would explain the measured small avalanche charges. A simulated charge spectrum without space charge effect is shown in Fig. 6.5. It has a monotonically decreasing shape as expected from the avalanche statistics (Eq. 2.14, see also Fig. (4). However, measurements show a shape that is very different (For example, see [39, 96]). 


\subsubsection{Timing RPCs with $0.3 \mathrm{~mm}$ Gaps}

A typical simulated avalanche in a $0.3 \mathrm{~mm}$ single gap Timing RPC at a high voltage of $3 \mathrm{kV}$ is shown in Fig. 6.6.

(a) In the first image at $t=0 \mathrm{~ns}$ the passage of a $7 \mathrm{GeV}$ pion generates three primary clusters: one consisting of one electron-ion pair, two with two electron-ion pairs each. The electrons are then drifting towards the anode at $z=0.3 \mathrm{~mm}$.

(b) The electrons in the cluster closest to the anode have left the gap at $t=0.17 \mathrm{~ns}$.

(c) The two remaining electron cluster grow further.

(d) The second electron cluster has left the gap at $t=0.42 \mathrm{~ns}$. We observe how the space charge begins to influence the electric field (compare to Fig. 1.1). The field is increased a little at the tip and at the tail of the last electron cloud in the gas gap while at its center it is about $15 \%$ lower. We find regions with increased multiplication but also regions with slower multiplication in the center where most of the electrons in that cluster are situated. Because of the resistivity of the anode layer all charges that reach the anode 'stick' to the electrode surface. Their presence influences the electric field in front of the anode.

(e) The closer the cluster approaches the anode, the higher the field at its tip gets. At $t=0.76 \mathrm{~ns}$ the maximum space charge field has a value that exceeds the applied electric field (at the tip of the avalanche) while in the center of the electron cloud the field is halved. Here we find strong attachment of electrons and a large amount of negative ions is formed.

(f) Due to the many electrons that have entered the resistive anode surface the field drops dramatically. From now on there is strong attachment of the remaining electrons in the gas gap and the drift velocity is small. The amount of negative ions in front of the anode grows rapidly.

(g) At $t=1.42 \mathrm{~ns}$ all electrons have either left the gap or got attached. The field in front of the anode is lowered to only $40 \%$ of the applied electric field.

(h) In the last image we show the induced current signal.

We calculated charge spectra of the induced and the total signal charge at different high voltages. In Fig. 6.7 we show spectra of the induced charge in a $0.3 \mathrm{~mm}$ single gap Timing RPC as in Fig. 4.1 b at high voltages of $2.3 \mathrm{kV}, 2.5 \mathrm{kV}, 2.8 \mathrm{kV}$ and $3.0 \mathrm{kV}$. We find values for the average charges that are quite close to the measurements. Here we also overlayed spectra where we assumed a conductive anode. In the case of the conductive anode, the signal charge is higher, since the charges that reach the anode disappear instantly and are therefore not contributing to the total field in the gas gap. 

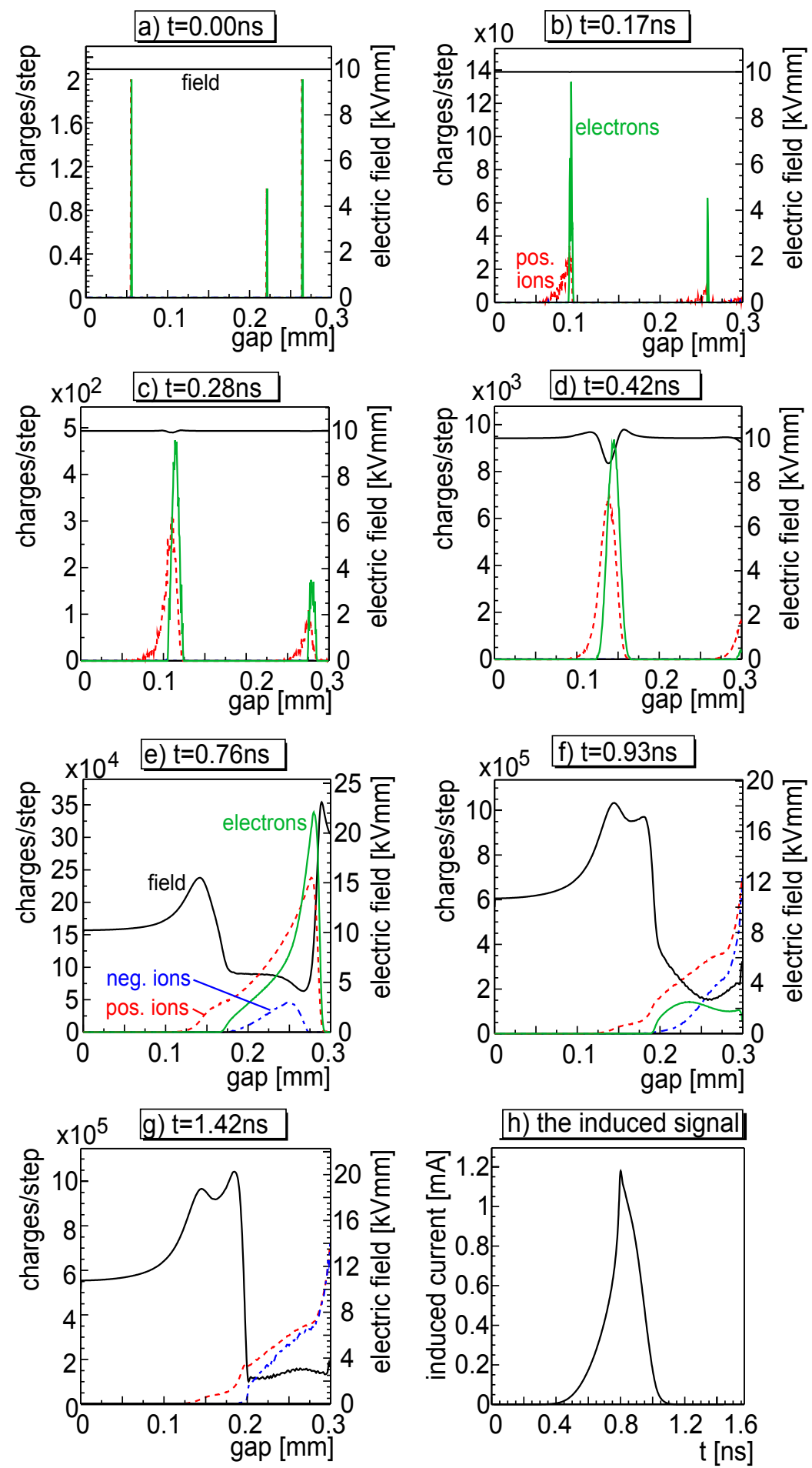

Figure 6.6: A simulated avalanche in a Timing RPC. We show snapshots of the charge configuration in a $0.3 \mathrm{~mm}$ gas gap (500 steps). The distributions of electrons, positive and negative ions are shown and correspond to the axes on the left. The $z$-components of the electric field across the gap is also plotted and correspond to the axes on the right. The last image is the induced current signal. 


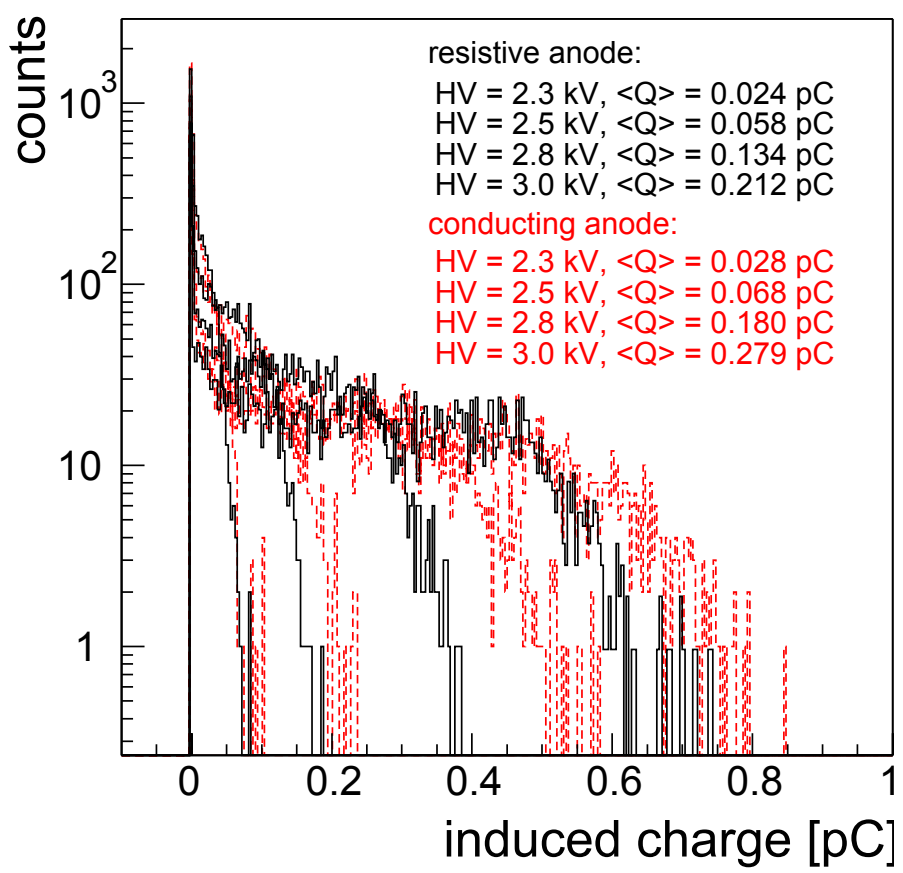

Figure 6.7: Comparison of simulated signal charge spectra for $0.3 \mathrm{~mm}$ single gap Timing RPCs with conductive and with resistive anode. The mean values take into account all data including the inefficient events. The temperature is $T=296.15 \mathrm{~K}$ and the pressure $p=970$ mbar.

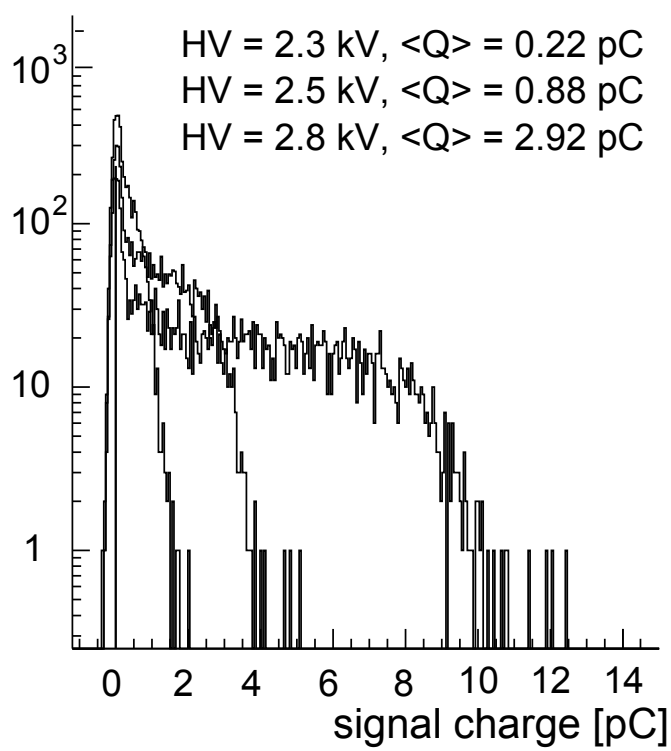

a) Simulation

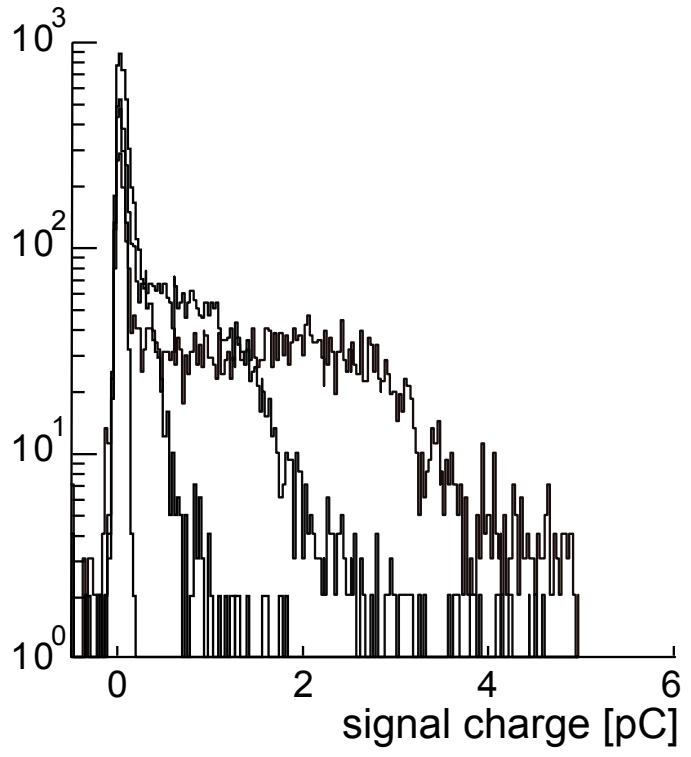

b) Measurement

Figure 6.8: a) Simulated signal charge spectra for $0.3 \mathrm{~mm}$ single gap Timing RPCs. The mean values take into account all data including the inefficient events. $100 \mathrm{fC}$ noise is added. b) Measured spectra from [96]. The temperature is $T=296.15 \mathrm{~K}$ and the pressure $p=970$ mbar. 
The difference in the average charges is in between $15 \%$ and $25 \%$. The difference is much smaller than one might expect from the large amount of charge that contributes to the generation of the space charge field in the one case and that does not contribute in the other. In experiment a difference is not observed [103].

In Fig. 6.8 we show simulated and measured spectra of the total signal charge in a $0.3 \mathrm{~mm}$ single gap Timing RPC as in Fig. 4.1 b at high voltages of $2.3 \mathrm{kV}, 2.5 \mathrm{kV}$ and $2.8 \mathrm{kV}$. We added $100 \mathrm{fC}$ noise to the values obtained with the simulation. The mean of the simulated spectra is a factor two larger than the measured one. But as compared with the discrepancy of nine orders of magnitude that we observed if a space charge is neglected (6.2) we can consider the simulation to be close to the measured values. Also the shape of the spectra is very similar to the measured data.

An uncertainty concerning the value of the Townsend and attachment coefficients $\alpha(E / p)$ and $\eta(E / p)$ at large electric field strengths is a possible reason for the small deviation of the average values of the spectra. It turns out that a decrease of the value of $\alpha(E / p)$ as it is shown in Fig. 2.8 by $10 \%$ leads to a decrease of the mean value of the spectra by $30 \%$. Since measurements of $\alpha(E / p)$ and $\eta(E / p)$ at the large values of the electric fields observed in RPCs are not available, an error of $10 \%$ or even more is certainly imaginable.

\subsubsection{Timing RPCs with $0.1 \mathrm{~mm}$ and $0.2 \mathrm{~mm}$ Gaps}

We simulated Timing RPCs with $0.1 \mathrm{~mm}$ and $0.2 \mathrm{~mm}$ gaps. Since the same gas mixture and the same materials are used, the parameters for the simulation are the same as with the $0.3 \mathrm{~mm}$ gap. Only the weighting field changes according to Eq. 2.40. Due to the thinner gaps the inefficiency is larger due to events that lead to no primary ionization in the gas gap. The most important simulation parameters are given in table 4.1.

In Fig. 6.9 we show simulated and measured spectra of the total signal charge in a $0.1 \mathrm{~mm}$ single gap Timing RPC at high voltages of $1.2 \mathrm{kV}, 1.4 \mathrm{kV}$ and $1.6 \mathrm{kV}$. We added $100 \mathrm{fC}$ noise to the values obtained with the simulation. The means of the simulated spectra differ from the measurements by a factor four. But again we remind that as compared with the huge discrepancy that we observe if a space charge is neglected we can consider the simulation to be close to the measurements. The shape of the simulated spectra is again very similar to the measured data from [96].

We also show simulated spectra of the total signal charge in a $0.2 \mathrm{~mm}$ single gap Timing RPC (Fig. 6.10). We used high voltages of $1.7 \mathrm{kV}, 1.9 \mathrm{kV}$ and $2.1 \mathrm{kV}$.

\subsubsection{Quad Gap Timing RPCs with $0.3 \mathrm{~mm}$ Gaps}

Now we consider a single gap RPC of any dimension. We assume a charge spectrum that has a certain shape with the mean value $\langle Q\rangle$. The efficiency of the gap is $\epsilon$ and 


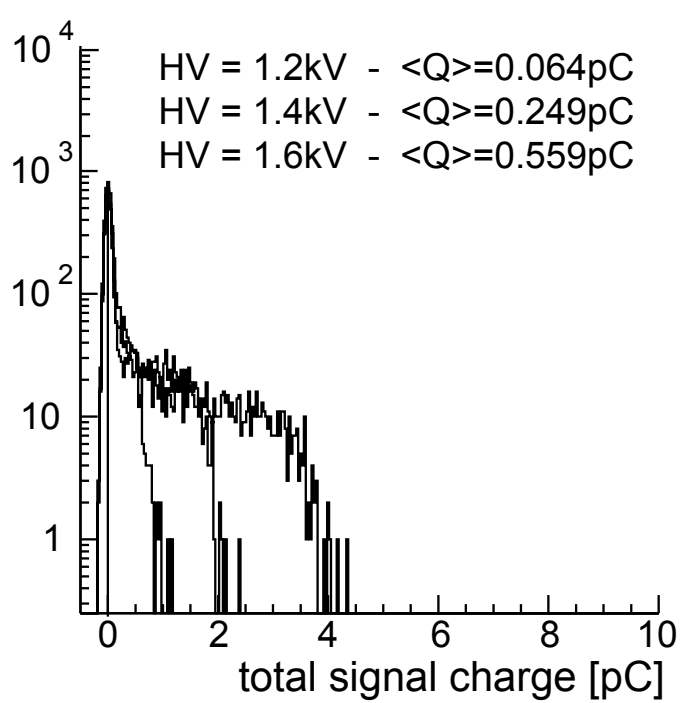

a) Simulation

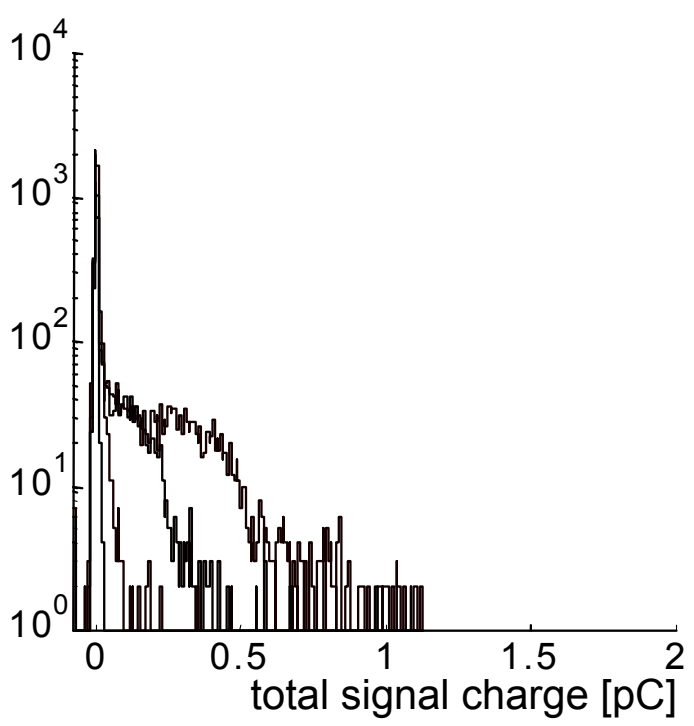

b) Measurement

Figure 6.9: a) Simulated signal charge spectra for $0.1 \mathrm{~mm}$ single gap Timing RPCs with the $\mathrm{C}_{2} \mathrm{~F}_{4} \mathrm{H}_{2} / \mathrm{i}-\mathrm{C}_{4} \mathrm{H}_{10} / \mathrm{SF}_{6}(85 \%, 5 \%, 10 \%)$ gas mixture. The mean values take into account all data including the inefficient events. $100 \mathrm{fC}$ noise is added. $\mathrm{b}$ ) Measured spectra from [96]. The temperature is $T=296.15 \mathrm{~K}$ and the pressure $p=970$ mbar.

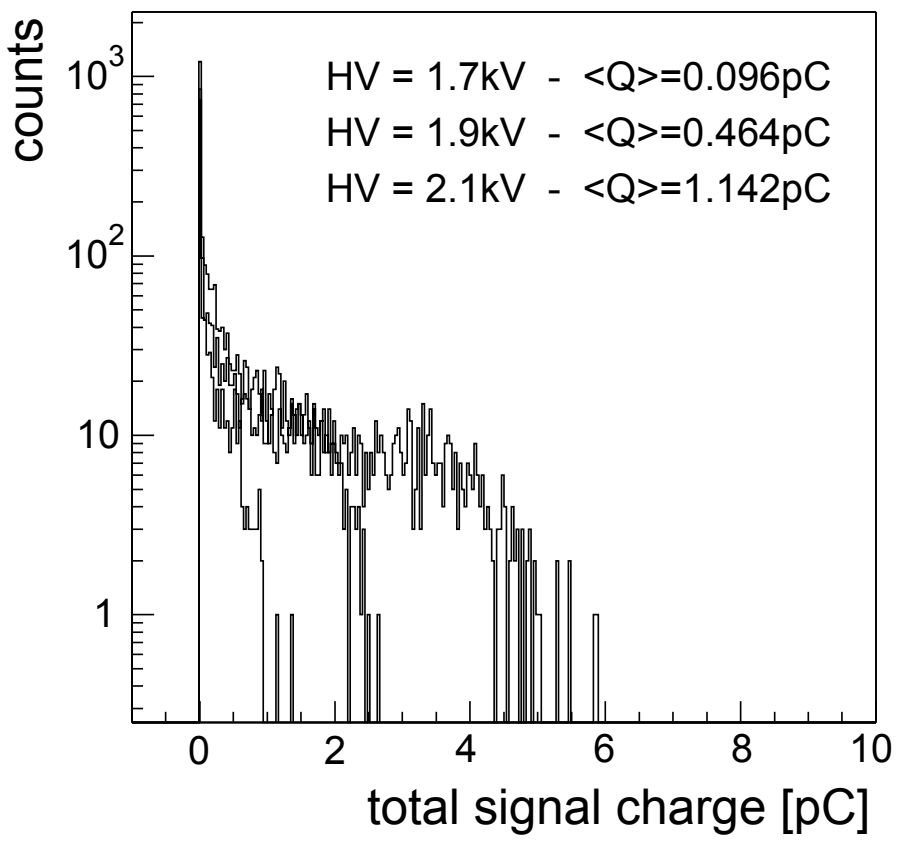

Figure 6.10: Simulated total signal charge spectra for $0.2 \mathrm{~mm}$ single gap Timing RPCs with the $\mathrm{C}_{2} \mathrm{~F}_{4} \mathrm{H}_{2} / \mathrm{i}-\mathrm{C}_{4} \mathrm{H}_{10} / \mathrm{SF}_{6}(85 \%, 5 \%, 10 \%)$ gas mixture. The mean values take into account all data including the inefficient events. The temperature is $T=296.15 \mathrm{~K}$ and the pressure $p=970 \mathrm{mbar}$. 


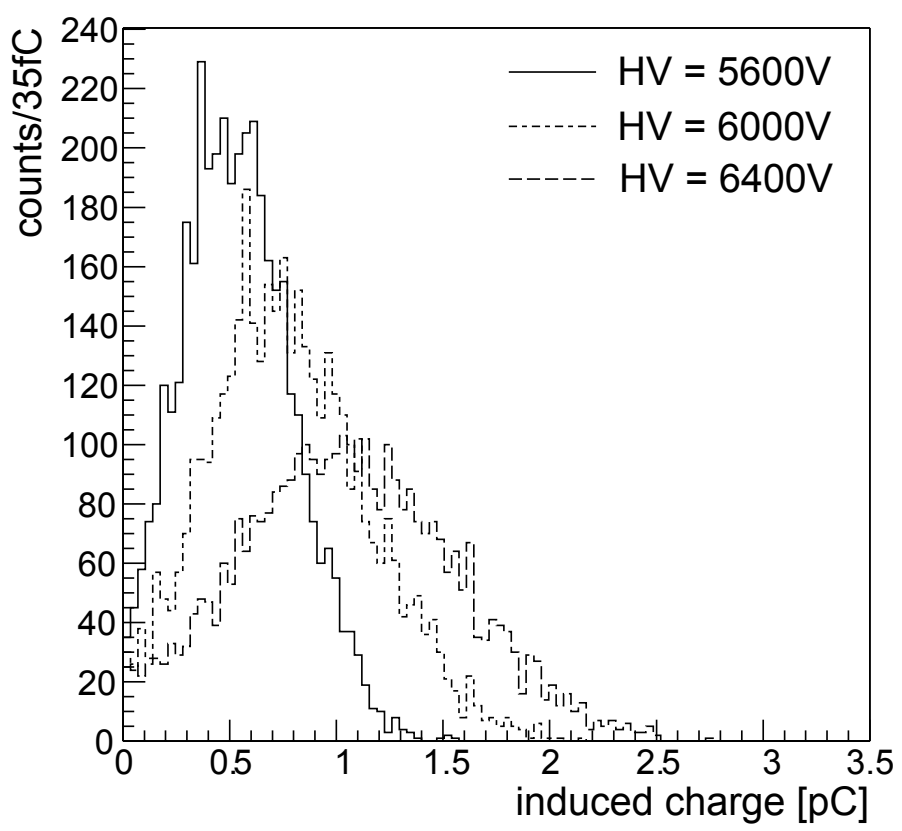

Figure 6.11: Simulated induced charge spectra for quad gap Timing RPCs. The temperature is $T=296.15 \mathrm{~K}$ and the pressure $p=970$ mbar.

the time resolution is $\sigma_{t}$. If we introduce $n$ gaps of the same type (gap width and gas mixture) the efficiency increases accordingly. It is important to remember that the weighting field generally changes in multi gap configurations. The weighting fields of an RPC like in Fig. 1.14 with $n$ gas gaps of size $g$ separated by $n-1$ glass plates of thickness $q$ and relative permittivity $\varepsilon_{r}$ is given by [1]

$$
E_{w}=\frac{\varepsilon_{r}}{n g \varepsilon_{r}+(n-1) q}, \quad n>1
$$

The efficiency of $n$ gaps increases like $1-(1-\epsilon)^{n}$ [1]], as one would expect. For the time resolution one would expect that it improves as $1 / \sqrt{n}$ with the number of gaps. However, this is not the case. If we consider as an example four gaps and assume that there is one electron avalanche in each of the gaps, then the avalanche with the fastest growth will dominate the time resolution, even though the signal is generated as a superposition of all the induced currents. The largest signal gives the earliest threshold crossing time, So the timing of the multi gap RPC is approximately given by the 'earliest gap'.

Now we investigate the charge spectrum of the multi gap RPC. While for the same weighting field the average charge in the $n$ gaps would be $n\langle Q\rangle$, it stays approximately constant due to the decrease of the weighting field as given by Eq. 6.4. However a peak is forming near the mean value and is becoming more pronounced at larger numbers 
of gaps. It is a consequence of the central limit theorem [104] that the sum of many independent identically distributed random variables gives a distribution that tends to be close to the normal distribution. As an example we show in Fig. 6.11 a simulated induced charge spectrum of a quad gap Timing RPC as in Fig. 4.1c. The spectra are equal to the four times self convoluted charge spectra of the single gap RPC from Fig. 6.7. The spectra of the quad gap chamber resemble quite well the ones presented in [97].

\subsubsection{Trigger RPCs with $2 \mathrm{~mm}$ Gaps}

A typical simulated avalanche in a $2 \mathrm{~mm}$ single gap Trigger RPC as in Fig. 1.11 at $10 \mathrm{kV}$ high voltage is shown in Fig. 6.12.

(a) At $t=0 \mathrm{~ns}$ the passage of a $120 \mathrm{GeV}$ muon generates 20 primary clusters with between one and three electron-ion pairs each. The electrons are then drifting towards the anode at $z=2 \mathrm{~mm}$.

(b) Some of the primary electrons get attached or enter the anode resistive layer so that at $t=1.42 \mathrm{~ns}$ there are only 13 clusters left.

(c) The space charge begins to influence the electric field at $t=5.6 \mathrm{~ns}$.

(d) At $t=7.34 \mathrm{~ns}$ the space charge effect is already quite strong. The space charge field reaches up to around $10 \%$ of the applied electric field strength.

(d) At $t=9.09 \mathrm{~ns}$ the space charge field reaches up to around $40 \%$ of the applied electric field strength.

(e) At $t=10.48 \mathrm{~ns}$ the field drops dramatically in a large fraction of the gas gap due to the large amount of negative charge that has entered the anode. From now on the field is very low everywhere where there are electrons; we find mainly attachment and the electron drift velocity is very slow.

(f) At $t=15.48 \mathrm{~ns}$ all electrons have either left the gap or got attached. The field in front of the anode is lowered to only $40 \%$ of the applied electric field.

(h) In the last image we show the induced current signal.

We accumulated spectra of the induced and signal charge for single gap Trigger RPCs at different high voltages $(9.5 \mathrm{kV}, 9.75 \mathrm{kV}$ and $10 \mathrm{kV})$. We chose 500 steps for the 1.5-D simulation procedure. In Fig. 6.13 we show the spectra of the induced charge and in Fig. 6.13 the spectra of the total signal charge At $10 \mathrm{kV}$ and even higher voltages, the simulation tends to be unstable and some events show an exploding electric field. If this behaviour is detected the simulation of the current event is stopped and 

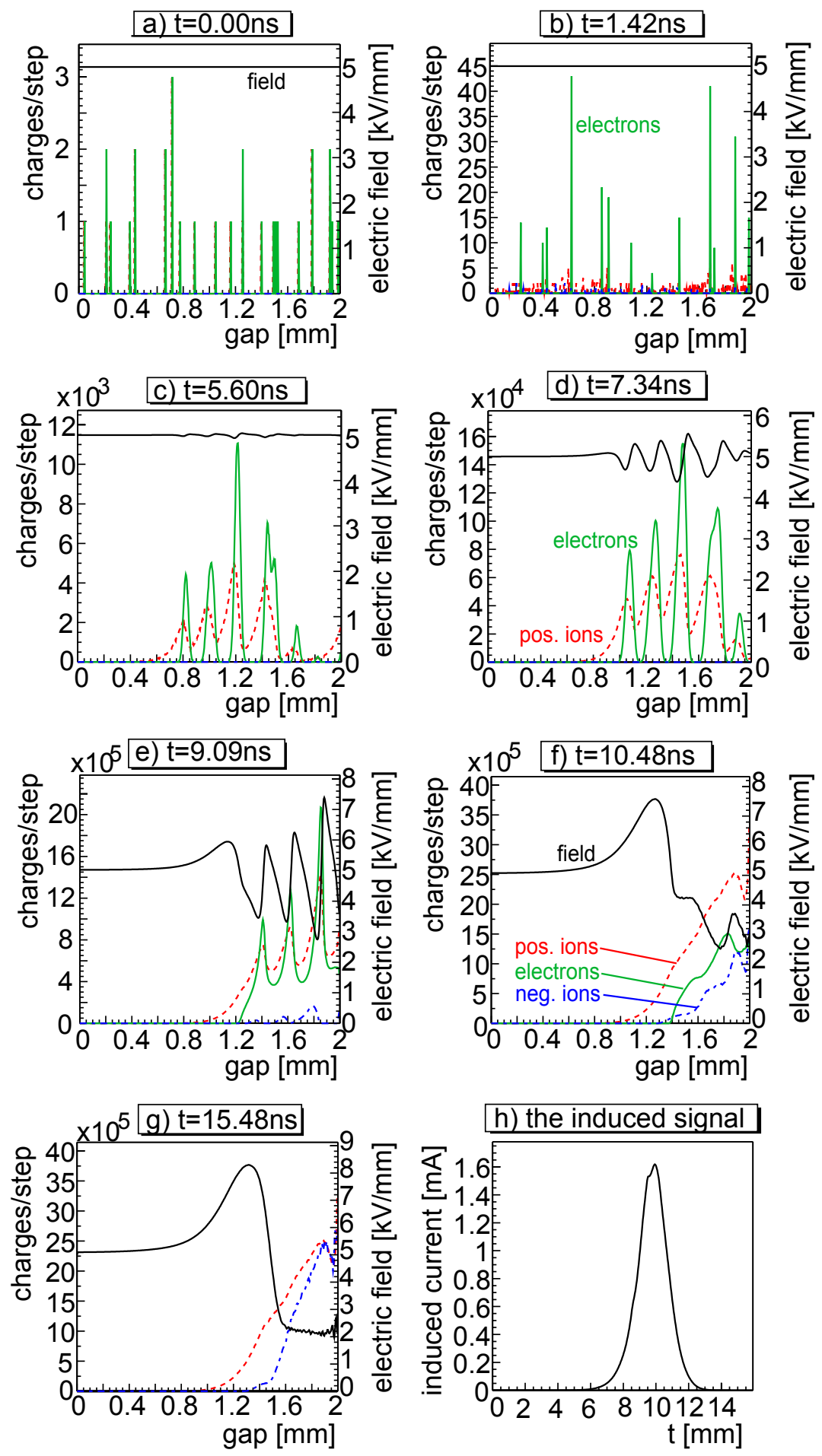

h) the induced signal

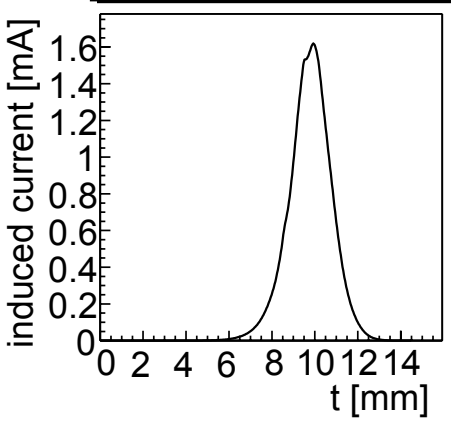

Figure 6.12: A simulated avalanche in a Trigger RPC. We show snapshots of the charge configuration in a $2 \mathrm{~mm}$ gas gap (500 steps). The distributions of electrons, positive and negative ions are shown and correspond to the axes on the left. The $z$-components of the electric field across the gap is also plotted and correspond to the axes on the right. The last image is the induced current signal. 


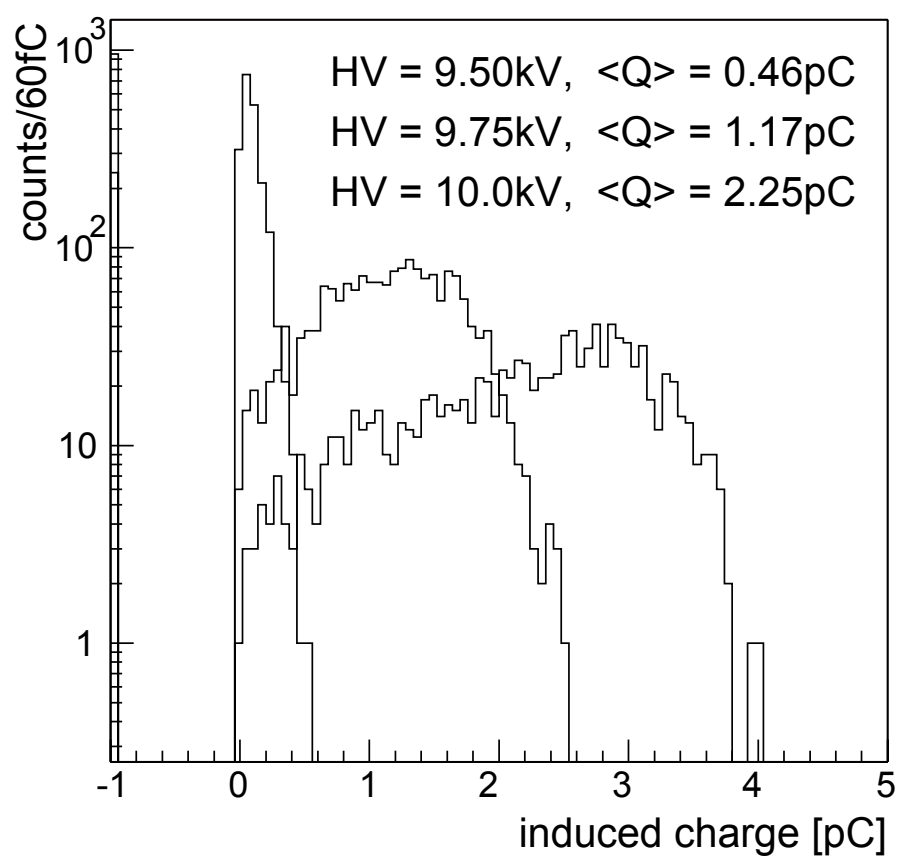

Figure 6.13: Simulated induced charge spectra for single gap Trigger RPCs with the $\mathrm{C}_{2} \mathrm{~F}_{4} \mathrm{H}_{2} / \mathrm{i}-\mathrm{C}_{4} \mathrm{H}_{10} / \mathrm{SF}_{6}(96.7 \%, 3 \%, 0.3 \%)$ gas mixture. The average values take into account only charges larger than a threshold of $100 \mathrm{fC}$.

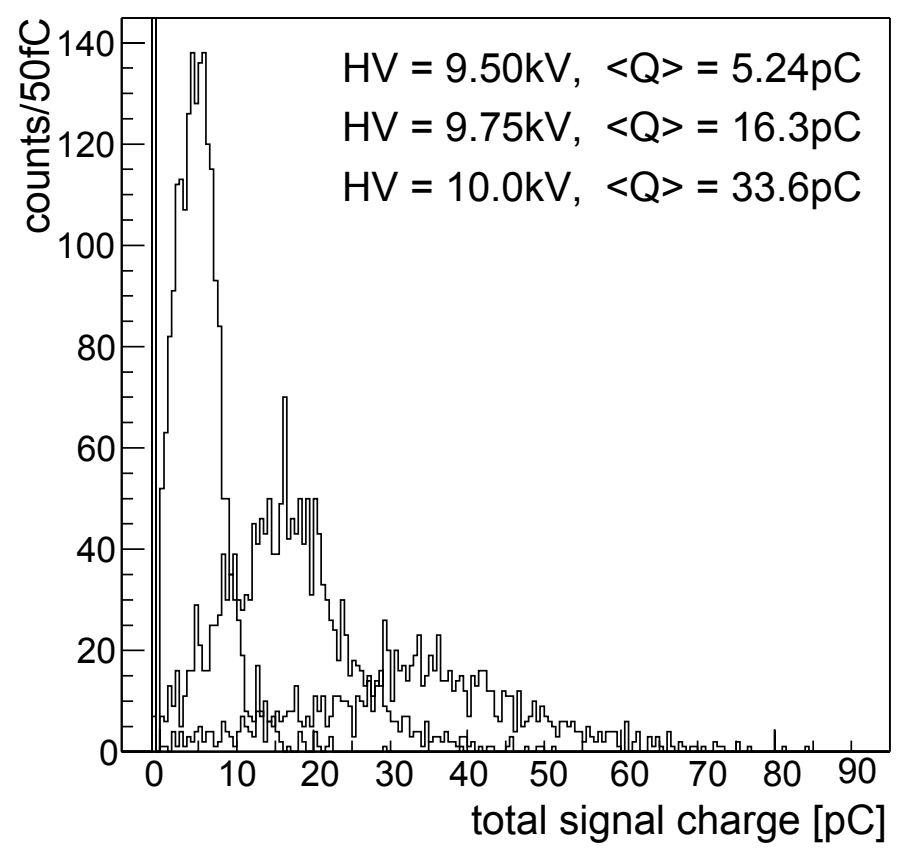

Figure 6.14: Simulated total signal charge spectra for single gap Trigger RPCs. The mean values take into account all data including the inefficient events. 


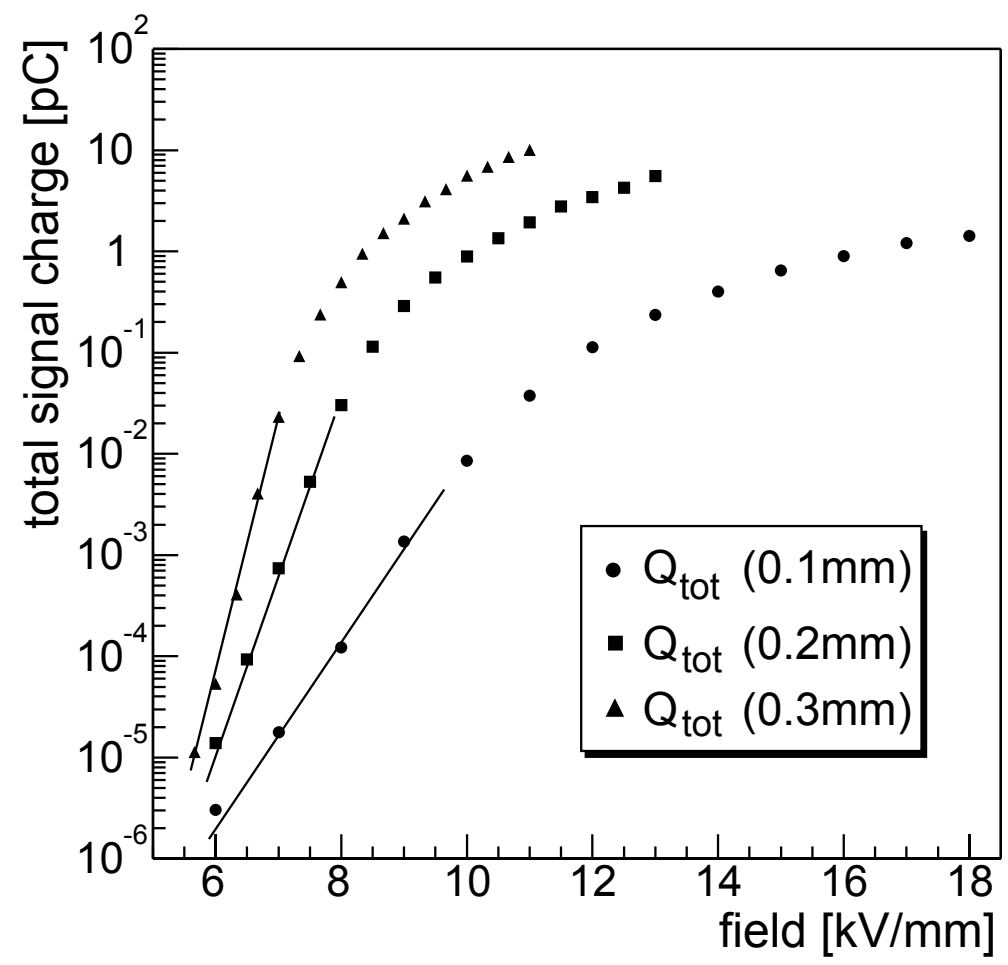

Figure 6.15: The average total signal charge of simulated avalanches in Timing RPCs with $0.1 \mathrm{~mm}$ to $0.3 \mathrm{~mm}$ gap widths versus the applied electric field. The solid lines are eye guides that correspond to exponential increase of the avalanche charges and evidencing the sub-exponential character of the data at higher fields (gain saturation).

skipped. The charges of these events are missing in the spectra and thus in the calculation of the average charges. Since the skipped events are generally events that would have given rather large charges, the average charges at $10 \mathrm{kV}$, that are calculated as $\left\langle Q_{\text {ind }}\right\rangle=2.25 \mathrm{pC}$ and $\left\langle Q_{\text {tot }}\right\rangle=33.6 \mathrm{pC}$, might be somewhat too small. Nevertheless, we find numbers for the average charges that are very similar to measurements. Also the shapes of the spectra are very similar to measured data (For example, see [39]).

\subsection{Operational Mode of RPCs}

From wire chambers filled with a quench gas with good UV absorption it is known that for certain high voltages one observes a region where the charge is proportional to the primary charge (proportional mode). Here the charge increases exponentially with the high voltage. After this one encounters the very narrow space charge mode of usually less than one hundred Volts where the charge growth deviates from the exponential. When further increasing the high voltage, the average charge suddenly increases by a 


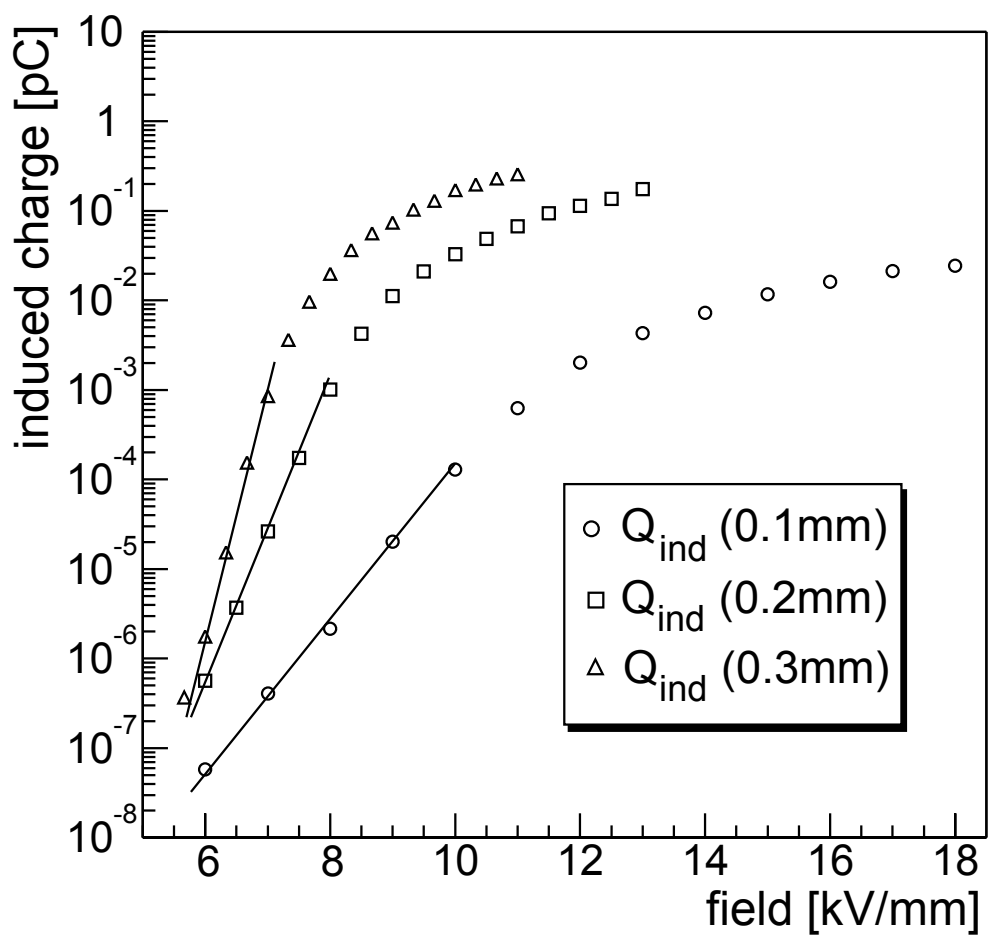

Figure 6.16: a) The induced charge of simulated avalanches in Timing RPCs with $0.1 \mathrm{~mm}$ to $0.3 \mathrm{~mm}$ gap widths versus the applied electric field. Again we show eye guides indicating the approximately exponential increase of the charges at low fields.

factor 10 to 100 (limited streamer mode). At even higher voltages, the charge continues to rise more slowly up to the general breakdown of the chamber or the Geiger-Müller mode [105, 106].

For parallel plate geometries like RPCs and neglecting space charge effects we expect an exponential dependence of the charge on the effective Townsend coefficient $\alpha_{e f f}=\alpha-\eta$. Since at high fields the dependence of $\alpha_{\text {eff }}$ on the field $E$ is approximately linear, the relation between the charge and $E$ will be approximately exponential, as in the wire chamber. As can be seen in Figs. 6.15 and 6.16, the Timing RPC shows this exponential behaviour at low fields, which is however giving charges that are too small for efficient operation. We also observe that in the broad operational region (in the case of the $0.3 \mathrm{~mm}$ gap Timing RPC from around 9 to $11 \mathrm{kV} / \mathrm{mm}$ ) the detector is operated in space charge mode.

The value of the charge depends first exponentially on the applied high voltage but then the dependence becomes approximately linear, which is also an observed experimental fact (For example, see Fig. 3 in [107] and Fig. 5 in [96]). Only at very high fields the occurrence of streamers is experimentally observed, which limits the space charge region towards higher voltages. 


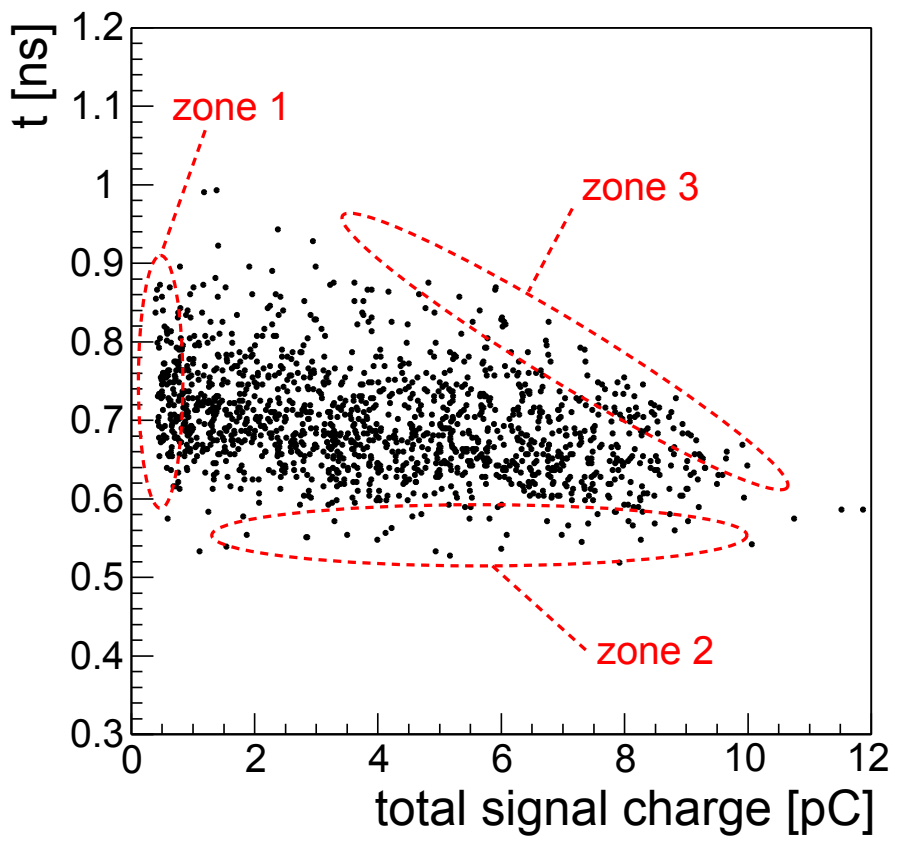

Figure 6.17: Intrinsic correlation of the charge threshold crossing time to the induced fast charge for simulated avalanches in a $0.3 \mathrm{~mm}$ single gap Timing RPC at $2.8 \mathrm{kV}$. The threshold applied to the induced charge was $10 \mathrm{fC}$. The temperature is $T=296.15 \mathrm{~K}$ and the pressure $p=970$ mbar.

\subsection{Charge-to-Time Correlation}

In section 5.2 we investigated the charge-to-time correlation of signals in Timing RPCs including read out electronics. Since, as mentioned there, a part of the correlation is introduced by the read out electronics, we also investigate charge-to-time correlations without electronics to show the intrinsic detector effects. In Fig. 6.17 we plot the time at which the threshold of $10 \mathrm{fC}$ is crossed by the signals versus the induced charge. We observe a triangular distribution that we will explain in the following. We will use the expression leading cluster, which refers to the cluster that is deposited at the position closest to the cathode. In general, the total signal charge is determined by the leading cluster because it has the longest drift distance and can thus reach the largest number of charge carriers. In Fig. 6.18 we show how the total signal charge is correlated to the position of the formation of the leading cluster. Obviously the signal charge is largest for avalanches with a leading cluster closer to the cathode. But let's come back to the charge-time correlation in Fig. 6.17. In the plot we have marked three zones that form the limits of the distribution:

Zone 1: The value of the threshold defines this limit. Avalanches that do not cross the threshold do not appear in the distribution. 


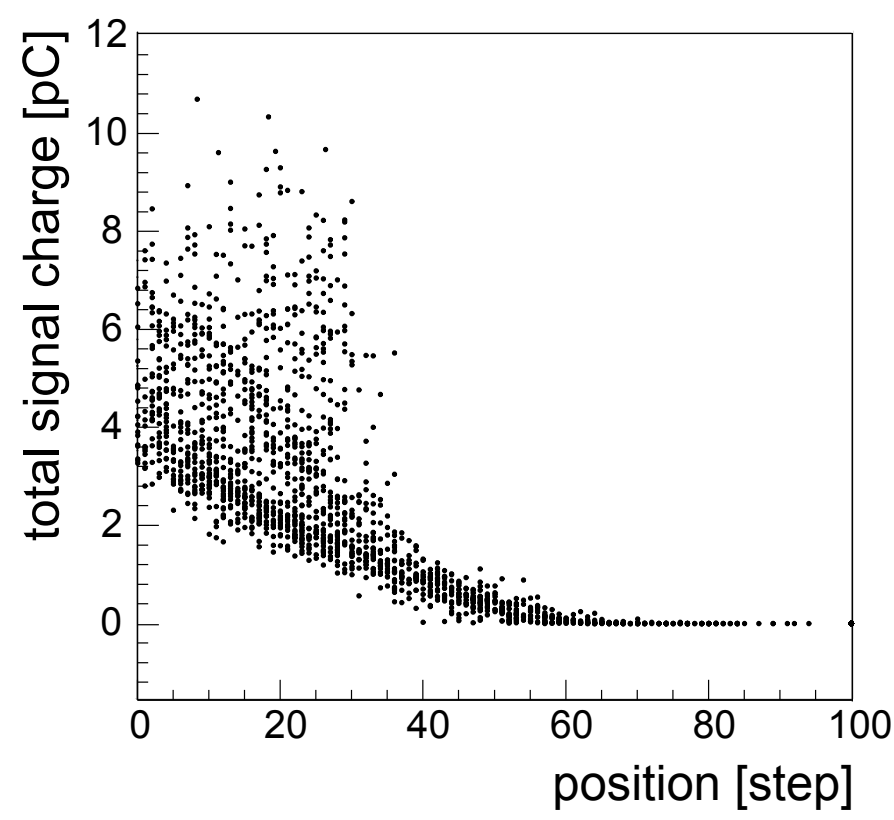

Figure 6.18: The correlation of the total signal charge to the position of the formation of the first cluster that reached the anode. We simulated avalanches in a $0.3 \mathrm{~mm}$ single gap Timing RPC at $2.8 \mathrm{kV}$.

Zone 2: Here we find the events with a very fast signal rise time. The fastest possible signal rise defines this limit. The signal rise time is determined by the avalanche statistics. From Fig. 3.1 we know that the very beginning of each avalanche determines its final size. Thus also the threshold crossing time is determined by the early stage of growth. In zone 2 we find no correlation of the threshold crossing time to the charge. To understand this we assume that the leading cluster determines the timing and that it is crossing the threshold very early. The leading cluster can be deposited at any position in the gas gap. According to Fig. 6.18, the avalanche charge will be very high if it is deposited close to the cathode. Correspondingly the charge will be very low if the leading cluster is deposited closer to the anode. It may just be large enough to cross the threshold.

Zone 3: Here we find the events with a slow signal rise time. We observe a clear correlation of the threshold crossing time to the charge. Towards higher values of the total signal charge the signal rise is becoming slower. Again we assume that the leading cluster determines the timing. The signals with the highest charges have a leading cluster that was deposited close to the cathode but also a fast signal rise time, meaning that the corresponding avalanches underwent a fast early growth. The maximum signal charge is limited by the space charge effect. 


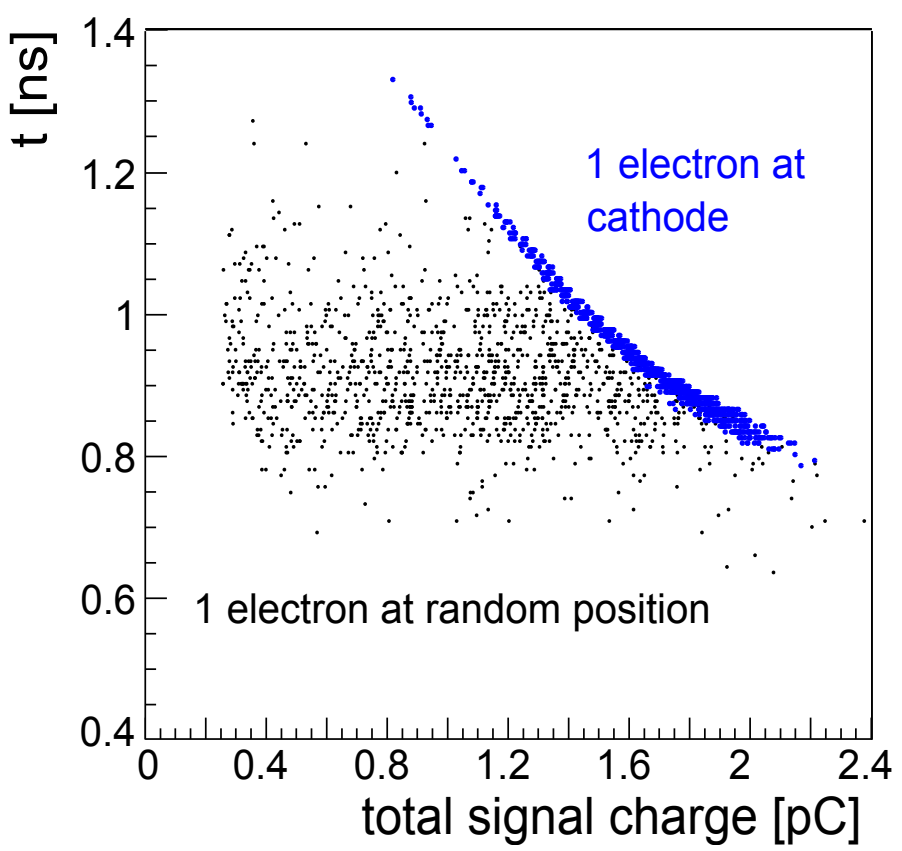

Figure 6.19: Simulated charge-time correlations for avalanches started by one electron, either at a random $z$-position in the gas gap or at the cathode at $z=0$.

On the other hand the events with the slowest signal rise time are connected to avalanches with lower values of the total signal charge. This can be understood if one imagines that an avalanche with the leading cluster close to the cathode has to undergo a very slow growth process in order to arrive at a final charge that is small.

To further illustrate the charge-to-time correlation we simulated avalanches that were started by one electron either at random $z$-position in the gas gap or right at the cathode at $z=0$. Fig. 6.19 shows that the largest values of the total signal charge are reached by avalanches that are started at the cathode and that undergo a fast initial growth process and therefore cross the threshold early.

\subsection{Avalanches in Pure Isobutane}

We also simulated timing RPCs with a $0.3 \mathrm{~mm}$ gap filled with pure isobutane. Since the attachment coefficient in pure isobutane is negligible, and since so far we observed a strong effect of attachment on the avalanche propagation in RPCs, we expect a very different behaviour. If we assume that the electric field strength sensed by some of the electrons in an avalanche in pure isobutane approaches zero, then the Townsend 

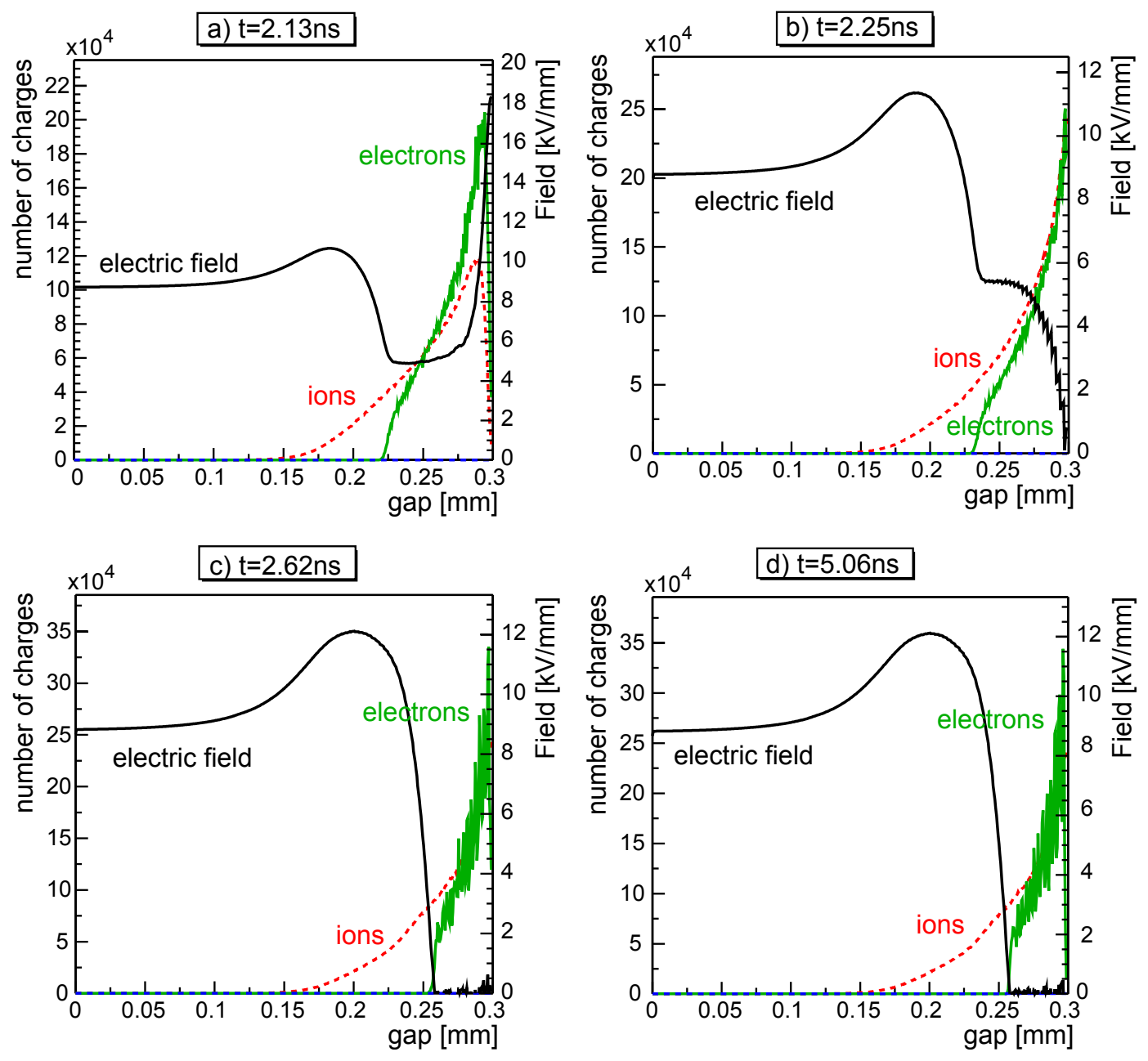

Figure 6.20: A simulated avalanche in a Timing RPC filled with pure isobutane. We show snapshots of the charge configuration in a $0.3 \mathrm{~mm}$ gas gap (500 steps).

coefficient" will also approach zero. Thus the avalanche size will not increase any more. On the other hand the number of electrons can only decrease if some electrons leave the gas gap.

In the case of a resistive anode the electrons that enter the anode can decrease the field in front of the anode such that the remaining electrons are drifting extremely slowly and are not multiplying. But on the other hand they also do not get attached. This creates the curious situation that some electrons stay in the gas gap 'forever'; they are trapped in the region of decreased electric field strength. This situation is shown in Fig. 6.20, where we plot the electric field and the charge distributions in an example simulated avalanche in a $0.3 \mathrm{~mm}$ gap Timing RPC filled with pure isobutane.

\footnotetext{
${ }^{1}$ The effective Townsend coefficient $\alpha_{\text {eff }}$ is in this case equal to the Townsend coefficient $\alpha$.
} 


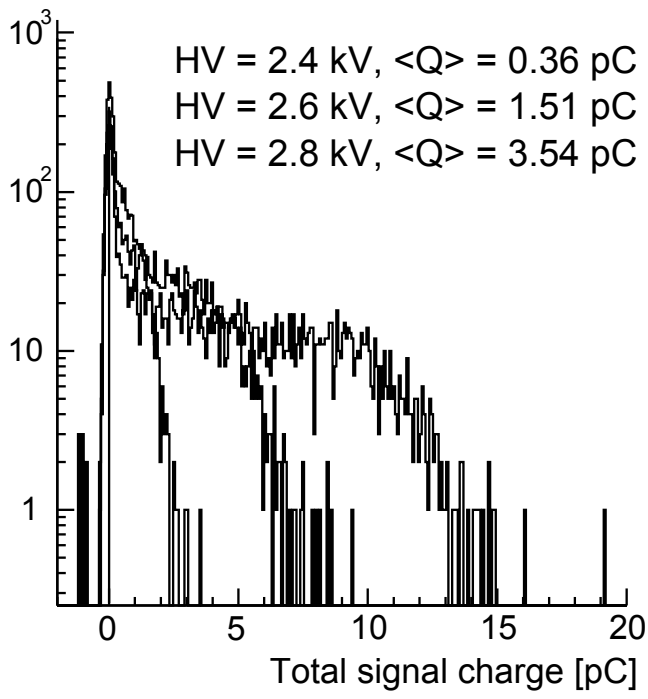

a) simulation

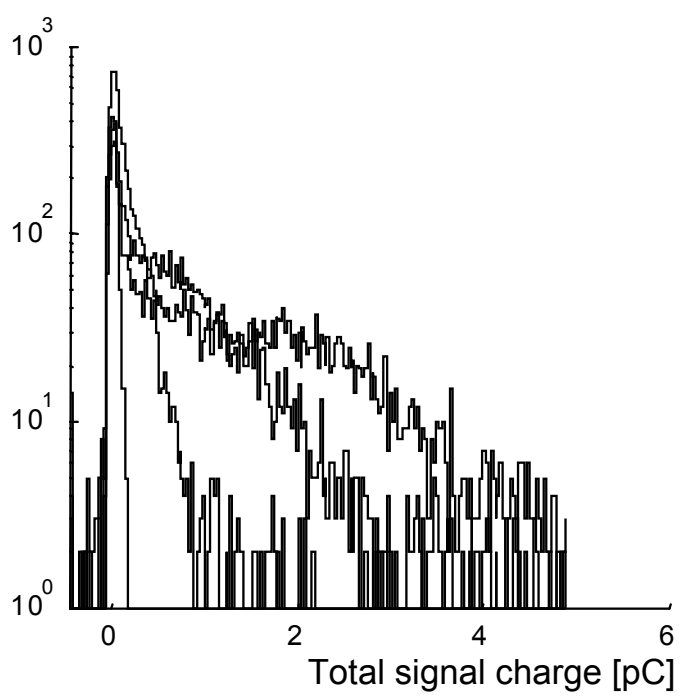

b) measurement

Figure 6.21: a) Simulated signal charge spectra for $0.3 \mathrm{~mm}$ single gap Timing RPCs filled with pure isobutane. The entries around a total signal charge of -1 correspond to events which were unstable. The mean values take into account all data including the inefficient events but excluding the unstable events. $100 \mathrm{fC}$ noise is added. b) Measured spectra from [96].

We voltage is set to $2.6 \mathrm{kV}$ and the avalanche is started by one electron at the cathode at $z=0$ and at $t=0$.

(a) At $t=2.13 \mathrm{~ns}$ the first electrons reach the anode. The electric field is almost doubled at the tip of the electron cloud.

(b) At $t=2.25 \mathrm{~ns}$ the field in front of the anode drops dramatically due to the amount of negative charge that 'sticks' to the anode resistive surface. Many electrons are still situated in regions where the drift velocity and the Townsend coefficient are larger than zero.

(c) At $t=2.62 \mathrm{~ns}$ all electrons are in the region where the field is approximately zero.

(d) At $t=5.06 \mathrm{~ns}$ about the same amount of electrons is still 'trapped' in the region of decreased electric field strength.

The reason for this effect is that the used model is a one dimensional one. The electrons can propagate only longitudinally along the $z$-axis. In a real avalanche also radial 'escape' possibilities are given. However, also in 'real' avalanches this effect 
will be present to some extend, because the fundamental physical processes that underly this curious behaviour are the same. To avoid the difficulties in our simulations, we assume a conducting anode, where no charges stick to its surface. Electrons entering the anode are draining off instantly. Only in some events at higher voltages the 'electron trap effect' does appear in that case. Since the number of trapped electrons in general is small compared with the peak value of electrons in the avalanche, we can assume that in those cases the total signal charge, which is the number of ions in the gas gap at the end of the signal, is not much affected.

Simulated charge spectra or Timing RPCs filled with pure isobutane and with a conducting anode at high voltages of $2.4 \mathrm{kV}, 2.6 \mathrm{kV}$ and $2.8 \mathrm{kV}$ are shown in Fig. $6.2 \mathrm{la}$. We added $100 \mathrm{fC}$ noise. As was the case in the simulation of avalanches in Trigger RPCs, some events are unstable. The percentage is below $0.1 \%$ here. The simulated spectra are compared to measured spectra from [96]. The mean values of the simulated spectra differ from the measurements by a factor of around three. Still we can consider the simulation results to be quite close to the measured values. The shapes of the spectra are very similar to the measurements.

\subsection{Streamers}

The phenomenon of streamers in RPCs was discussed in section 2.5. Anode streamers form at the tip of a moving cluster of electrons in an avalanche while cathode streamers form at its tail [93]. The presence of a big space charge is a necessary requirement for the development of a streamer. This space charge can be either ions from previous avalanches, that have not yet left the gas gap or the charge carriers of the avalanche itself. The second phenomenon leads to the avalanche itself being the cause of its instability. The field of its space charge at some point exceeds some critical value so that the more or less well regulated avalanche propagation transforms into a streamer. When streamers reach both electrodes a channel of high conductivity can be formed between the electrodes, leading to a discharge (spark) in the RPC that is however localized due to the resistivity of the electrodes [40].

Fig. 6.22a shows the number of electrons in avalanches started by one electron at the cathode in a Timing RPC gas gap at different high voltages. Fig. 6.22b shows the evolution of the peak value of the $z$-component of the electric field in the same avalanches. The maximum is reached just before the electron cloud reaches the anode. From that point on the highest field is present at the tail of the electron cloud. After the electrons have left the gas gap, their presence in the resistive anode layer and the ions in the gap still alter the electric field in the gap. The electric field can easily reach double or three times the value of the applied electric field $E_{0}$. At very high fields, where measurements show a significant streamer probability, the simulations still show a saturated avalanche. Measurements show that at applied field strengths of around $10 \mathrm{kV} / \mathrm{mm}$ in four gap Timing RPCs, streamers occur with a probability of around 

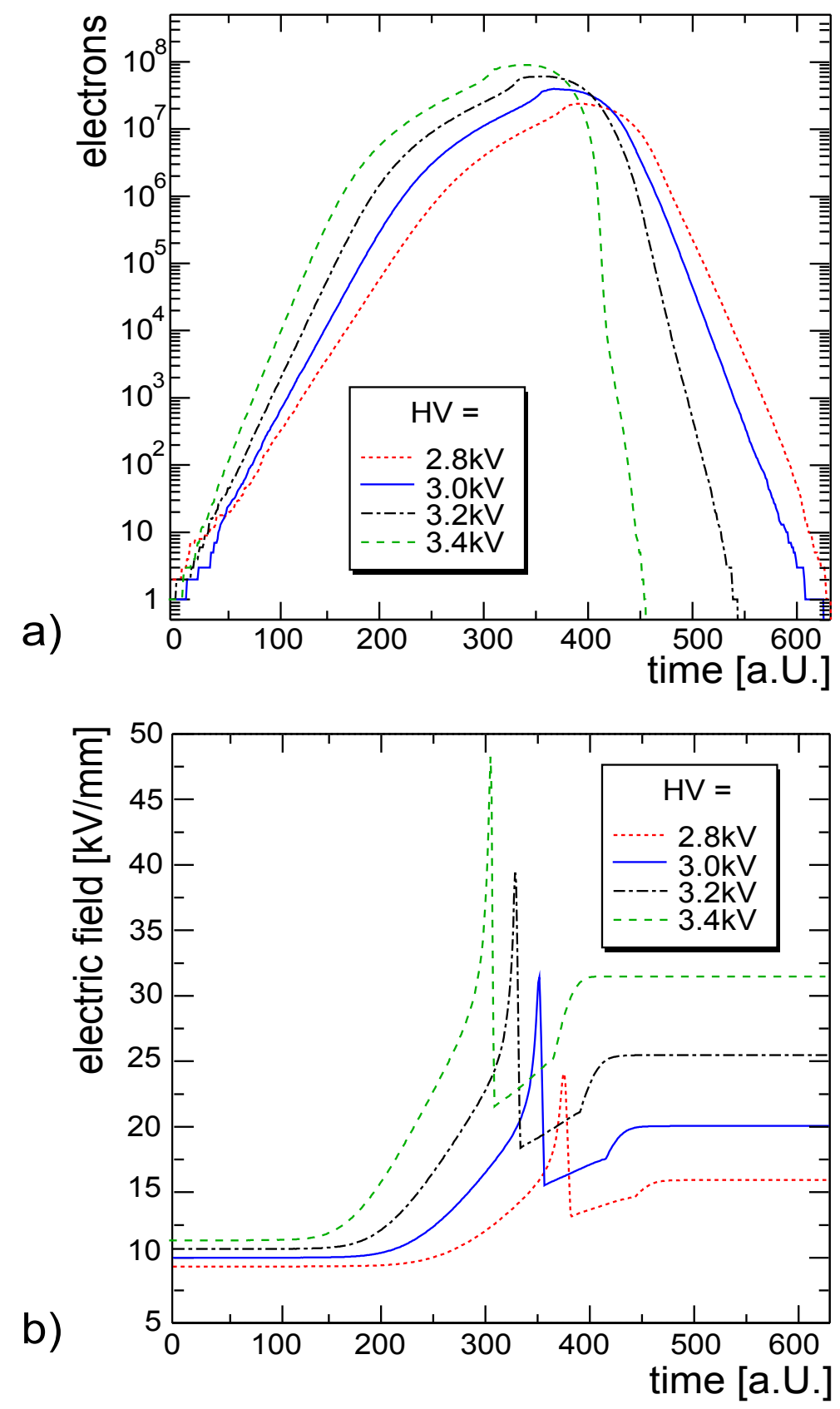

Figure 6.22: a) The total number of charge carriers in simulated avalanches started by one electron at the cathode for different high voltages. b) The maximum value of the electric field in the same avalanches as in a). 100 time steps correspond to about $0.5 \mathrm{~ns}$. 
$1 \%$, typically releasing a pulse of $20 \mathrm{pC}[69,97]$. At higher voltages the streamer probability is growing up to $25 \%$ at around $11 \mathrm{kV} / \mathrm{mm}$. Therefore, the quantitative description of the avalanche-to-streamer transition is not reproduced by our model. Note that no photonic effects have been included.

\subsection{Summary}

We presented results on the simulation of avalanches in Resistive Plate Chambers using the 1.5-D model. We summarize:

- Neglecting space charge effects the expected average charges are several orders of magnitude larger than the measured values. Simulated charge spectra show a monotonically decreasing shape, as expected from the statistics of the primary ionization and the avalanche multiplication, while measured spectra show a very different shape.

- Including the space charge effect in the simulation we obtain charge spectra that have a shape very similar to measured spectra. The mean values only differ by a factor of two to four. The space charge effect reduces the induced charges in Timing RPCs (Trigger RPCs) by a factor of $10^{7}\left(10^{3}\right)$ and the total signal charges by a factor of $10^{9}\left(10^{5}\right)$.

- At the threshold level, the space charge effect already influences strongly the signal rise. The time resolution is not affected by this process.

- Contrary to wire chambers, RPCs operate in a space charge mode that is very broad. The experimental result of a first exponential and then linear dependence of the average charges on the high voltage is reproduced by the simulations.

- The charge-to-time correlation is partly influenced by the read out electronics and by intrinsic detector effects. Intrinsically the signals with a slow rise show a correlation to the avalanche charge.

- Attachment plays a very important role in the development of avalanches in RPCs filled with an electronegative gas.

- As expected, the simulations do not reproduce quantitatively the avalanche-tostreamer transition, because no photonic effects are included. 


\section{Chapter 7}

\section{Results Obtained with the 2-D Model}

In this chapter we present results on the detailed simulation of single avalanches in $0.3 \mathrm{~mm}$ gap Timing RPCs using more dimensional models. We start with a comparison of the different simulation models that implement the dynamic calculation of the space charge field (section 7.1). Then we show results of detailed simulations with the 2D model that was described in section 3.3, since in section 3.4 it turned out that with the 3 -D model one has to use a very small binning which leads to the program to be too time consuming for the simulation of whole avalanches. We will focus on single gap Timing RPC with $0.3 \mathrm{~mm}$ gaps. As we saw in chapter 5, the space charge effect is much more prominent in this type of RPC. For our studies we use the device that is shown in Fig. 4.1 a as an example, where one electrode is made of resistive glass and the other of aluminum. The avalanches are always started by a single electron at the cathode. In section 7.2 we focus on avalanches in a gap filled with the standard gas mixture $\mathrm{C}_{2} \mathrm{~F}_{4} \mathrm{H}_{2} / \mathrm{i}-\mathrm{C}_{4} \mathrm{H}_{10} / \mathrm{SF}_{6}(85 \%, 5 \%, 10 \%)$. In section 7.3 we study avalanches in a detector with the same geometry but filled with pure isobutane $\left(\mathrm{i}-\mathrm{C}_{4} \mathrm{H}_{10}\right)$.

\subsection{Comparison of the Different Models}

In this section we compare the results of the three different simulation models that implement the calculation of the space charge field. They were described in sections 3.2, 3.3 and 3.4. Fig. 7.1a shows the development of the total number of electrons in random avalanches started by one electron at the cathode for the three different models. We simulated a $0.3 \mathrm{~mm}$ single gap Timing RPC at $2.8 \mathrm{kV}$. The gas gap is filled with the standard Timing RPC gas mixture. In all models the $z$-axis is divided in 300 steps. In the 2D model the $r$-coordinate is divided in 150 steps and in the 3-D model the $x$ - and $y$-axes are divided in 100 steps each. The avalanche simulated with the 3-D model was simulated as far as a reasonable time allowed (around $2 \times 10^{6}$ electrons). The timescales for the simulation of single avalanches is around a minute for the 1.5-D 

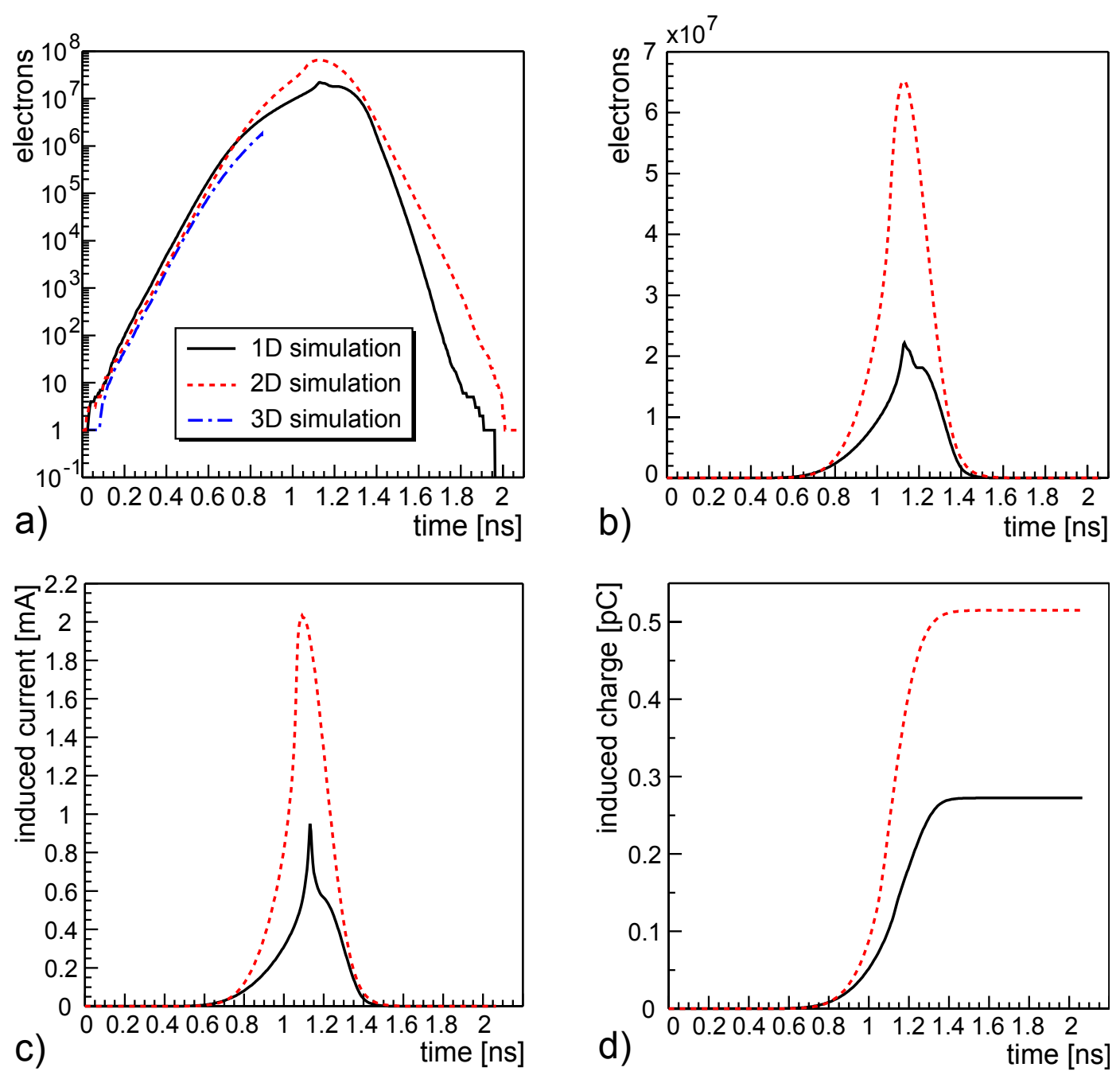

Figure 7.1: Comparison of three random avalanches that were simulated with the different models that implement the space charge effect. We simulated a Timing RPC at a high voltage of $2.8 \mathrm{kV}$ filled with the standard gas mixture $\mathrm{C}_{2} \mathrm{~F}_{4} \mathrm{H}_{2} / \mathrm{i}-\mathrm{C}_{4} \mathrm{H}_{10} / \mathrm{SF}_{6}$ $(85 \%, 5 \%, 10 \%)$ at $T=296.15 \mathrm{~K}$ and $p=1013$ mbar. The avalanches were started by one electron at the cathode. In all models we used a division of the $z$-axis into 300 steps. In the 2D model the $r$-coordinate is divided in 150 steps and in the 3-D model the $x$ - and $y$-axes are divided in 100 steps each. a) The total number of electrons versus time. b) The same plot on a linear scale. c) The induced current versus time. d) The induced charge versus time. 
model, around a day for the 2-D model and several days for the 3-D model up to the mentioned number of electrons.

In Figs. 7.1 a and $\mathrm{b}$ we observe that the initial growth is very similar for the three different models. The avalanches simulated with the 1.5-D and the 2-D models reach their maxima at the same time step. At the stage where the number of electrons is large, the growth rate is suppressed to a larger extend in the avalanche that is simulated with the 1.5D model. Even though the initial growth of this avalanche was stronger, the maximum number of electrons is only around a third of the number that is obtained with the 2-D model. The saturation effect is stronger in the 1.5-D case. Two reasons can be given:

- In the 1.5-D model we calculate the $z$-component of the electric field of radial Gaussian charge distributions. As was mentioned in section 3.2, it is always calculated at $r=0 \mathrm{~mm}$, in the center of the avalanche. However, here the field has the largest value. In a real avalanche electrons are also situated at other positions with $r \neq 0$, where the field is less strong. This means that in the simulation we calculate a space charge field that is somewhat too strong.

- As we shall see in sections 7.2.1 and 7.2.8, the electron clouds are distorted transversely by the attractive and/or repulsive space charge fields. In the 1.5-D model, on the other hand, the standard deviations $\sigma$ of the radial Gaussian charge distributions depend only on the transverse diffusion. If we accept that the radial repulsion of the electrons in the center and at the tip of the electron clouds leads to an increase of the radial spread of the avalanche, then $\sigma$ is chosen too small. Thus we will overestimate the radial charge density in the 1.5-D model. As we showed in Fig. 3.6, the $z$-component of the electric field of radial Gaussian charge distributions with different $\sigma$ can differ considerably. As as a result, the space charge effect is overestimated in the 1.5-D model.

After the maximum is reached, the electron number decreases due to attachment and the fact that electrons enter the anode and leave the gas gap (compare to Fig. 6.6). The decline of the electron number is faster in the case of the 1.5-D model. Here propagation is only allowed longitudinally along the $z$-axis. Since the calculated space charge fields are stronger in the 1.5-D model than in the 2-D model, we will find a larger region with strong attachment here.

The induced current signal and the development of the induced charge are shown in Figs. 7.1 $\mathrm{c}$ and $7.1 \mathrm{~d}$. The charge that is induced by the avalanches are $0.27 \mathrm{pC}$ for the 1.5-D model and $0.52 \mathrm{pC}$ for the 2-D model. The difference is about a factor of two. The charge of the ions in the gas gap at the time when the electrons have all either entered the anode or got attached $]^{1}$ is $1.79 \mathrm{pC}$ for the 1.5 -D model and $4.05 \mathrm{pC}$ for the 2-D model.

\footnotetext{
${ }^{1}$ This is the number of positive ions minus the number of negative ions. It is not to be confused with the total signal charge $Q_{t o t}$ that is given by the number of positive ions.
} 


\subsection{Avalanches in $\mathbf{C}_{2} \mathbf{F}_{4} \mathbf{H}_{2} / \mathbf{i}-\mathbf{C}_{4} \mathbf{H}_{10} / \mathbf{S F}_{6}$}

In this section we present results of the detailed simulation of single avalanches in $0.3 \mathrm{~mm}$ single gap Timing RPCs. We use the geometry shown in Fig. 4.1 a. We assume that the anode is made of aluminum and use the 2-D model that is described in section 3.3. As was mentioned there, the dependency of the values of the gas parameters (Townsend and attachment coefficients, diffusion coefficients and drift velocity) are taken from Figs. 2.8, 2.5, 2.4. The gas mixture is $\mathrm{C}_{2} \mathrm{~F}_{4} \mathrm{H}_{2} / \mathrm{i}-\mathrm{C}_{4} \mathrm{H}_{10} / \mathrm{SF}_{6}(85 \%, 5 \%$, $10 \%$ ) and the applied high voltage of $2.8 \mathrm{kV}$ leads to an electric field of $93.3 \mathrm{kV} / \mathrm{cm}$ in the gas gap. The pressure is 1013 mbar and the temperature $296.15 \mathrm{~K}$. Each avalanche is started by one electron at the cathode $(r=0, z=0)$.

\subsubsection{Electron Density}

Fig. 7.2 shows the electron distribution in an avalanche that was started by a single electron at the position $r=0, z=0$ and at $t=0 \mathrm{~ns}$. From the cathode the electron crosses the whole gap and reaches a maximum size of almost $4 \times 10^{7}$ electrons.

(a) On the first image at $t=1.0 \mathrm{~ns}$ we observe that the shape of the distribution differs from a strictly Gaussian shape. It is very similar as the shapes that were obtained with the 1-D model (for example, see Fig. 3.8). At the tip of the distribution the multiplication is stronger and the electron density is increased. At the tail the electron density is decreased.

(b) At $t=1.05 \mathrm{~ns}$ the peak of the electron distribution reaches the anode. The actual drift velocity in the gas and at the applied field strength would lead to a drift time of $t_{D}=0.3 \mathrm{~mm} /(0.2 \mathrm{~mm} / \mathrm{ns})=1.5 \mathrm{~ns}$. Nevertheless, diffusion combined with the repulsive space charge field that acts on the electrons at the tip of the distribution lead to an acceleration of the electrons there.

(c) At 1.19 ns many electrons have already entered the conductive anode. The shape of the distribution changes dramatically from now on.

(d) At $1.29 \mathrm{~ns}$ the electrons close to the anode disappear rapidly. As we already saw in section 6.2 they are attached to the electronegative gas components due to the lowered field in this region.

$(e, f)$ The electrons leave the gas gap or get attached. The electron density in the center of the avalanche at small values of $r$ decreases faster than further 'outside'.

Fig. 7.3 shows a contour plot of the electron density in the cluster of electrons that moves towards the anode at $z=0.03$. While in the first image at $t=0.5 \mathrm{~ns}$, where the avalanche is still relatively small $\left(\approx 8 \times 10^{5}\right.$ electrons $)$, the shape of the distribution 

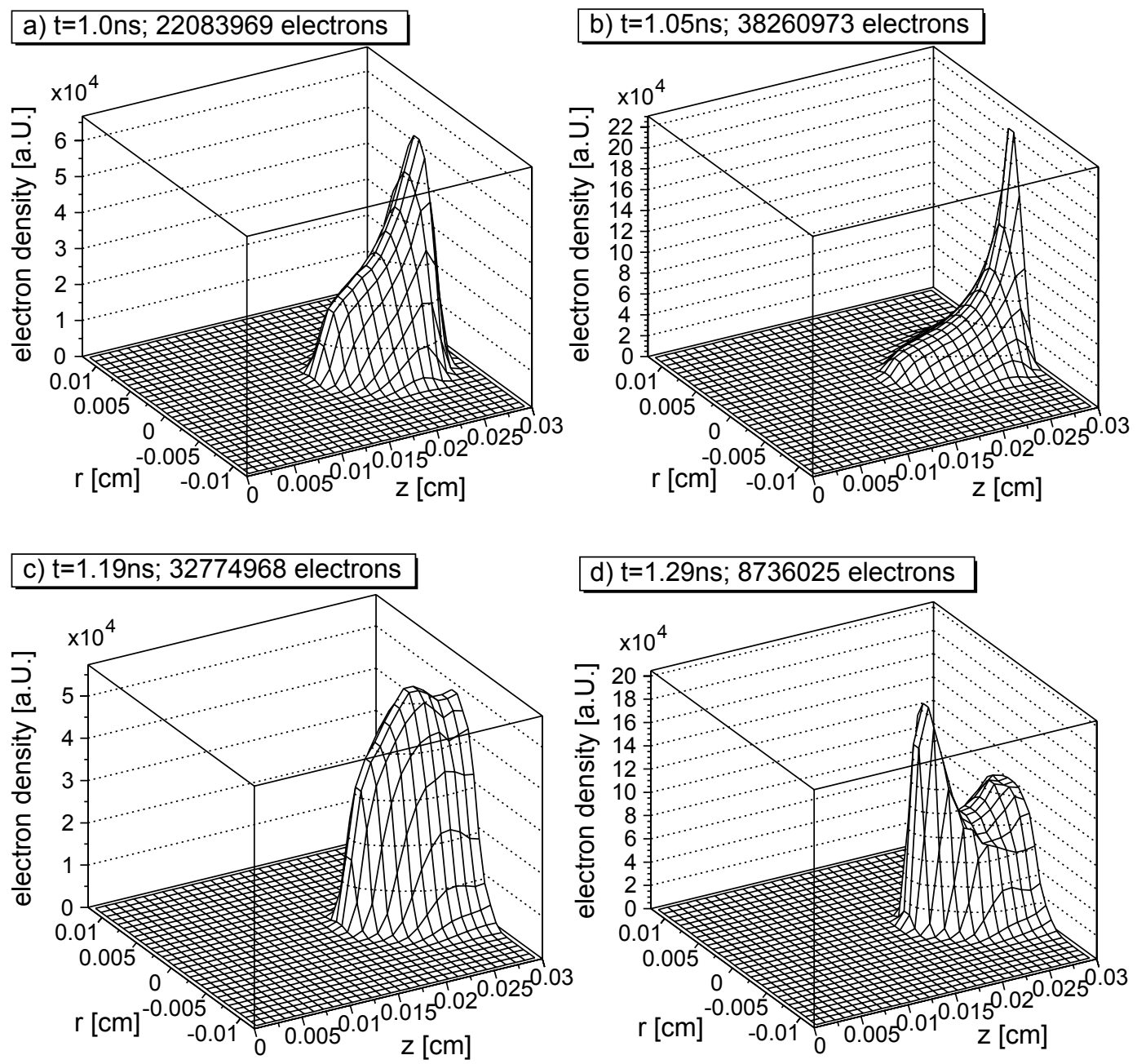

\section{e) $t=1.38 n s ; 1134153$ electrons}
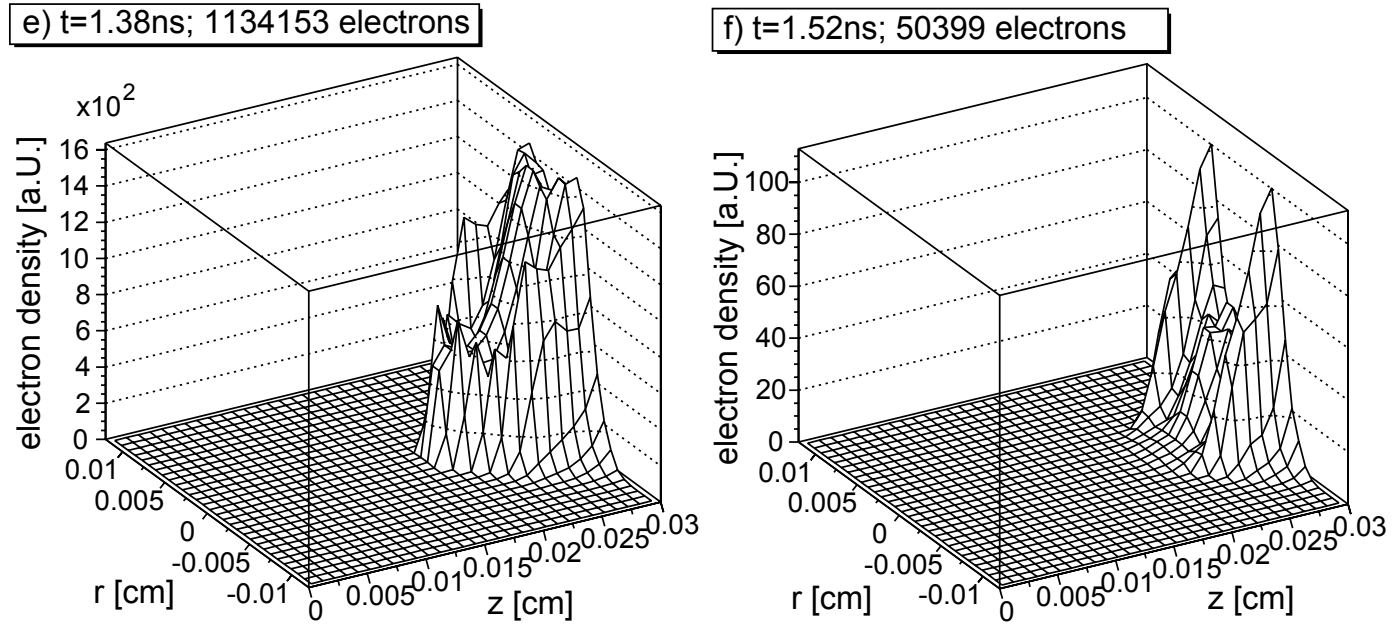

Figure 7.2: Electron density in an avalanche in a $0.3 \mathrm{~mm}$ gap Timing RPC filled with the standard gas mixture and with an operating voltage of $2.8 \mathrm{kV}$. The avalanche was started by one electron at $(r=0 \mathrm{~mm}, z=0 \mathrm{~mm})$. 

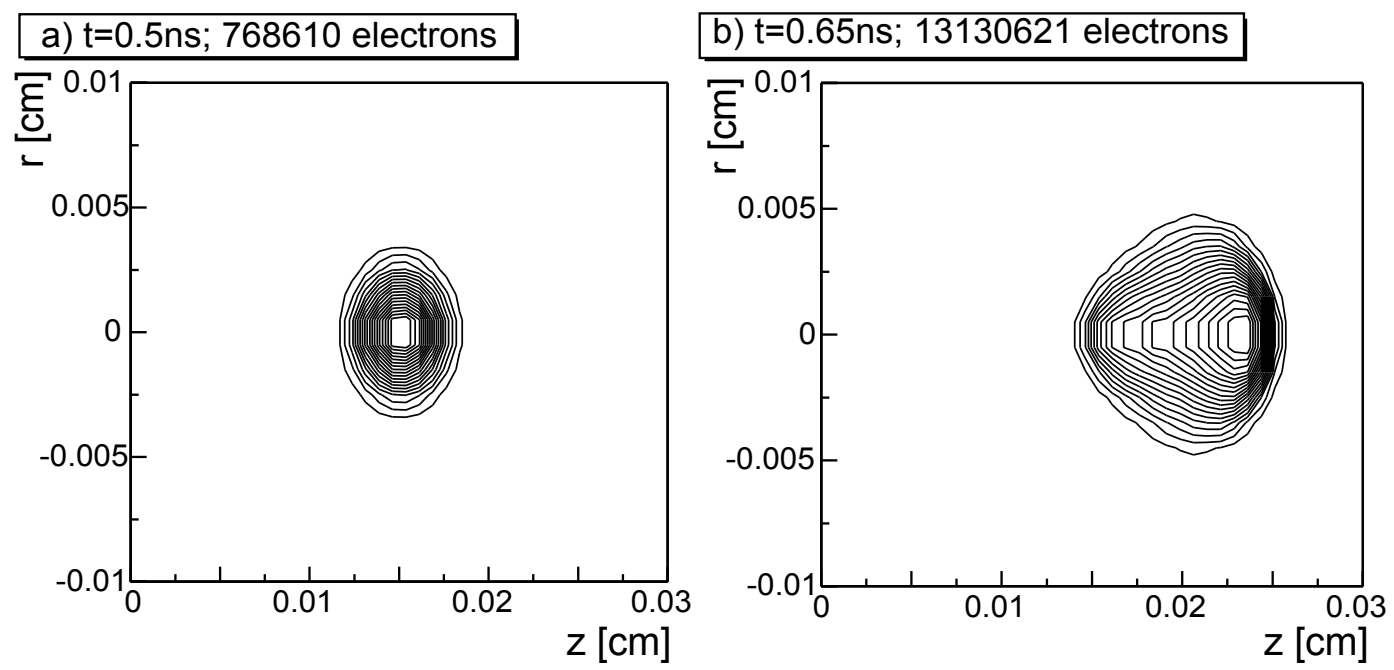

Figure 7.3: Electron density in an avalanche in a $0.3 \mathrm{~mm}$ gap Timing RPC filled with the standard gas mixture and with an operating voltage of $2.8 \mathrm{kV}$.

is roughly symmetric, it is different in the second image at $t=0.65 \mathrm{~ns}$. Here the avalanche has reached a size of about $1.3 \times 10^{7}$ electrons and the electrons at the tip of the electron cloud are repelled from the center at $r=0$ by repulsive space charge fields, while at the tail of the distribution they are attracted by a field of opposite sign. We shall investigate the radial space charge fields in more detail in section 7.2.8.

\subsubsection{Total Ion Density}

We now focus on the ion distribution. Fig. 7.4 shows the total ion density in an avalanche that was started by a single electron at the position $r=0, z=0$ and at $t=0 \mathrm{~ns}$. We show the number of positive ions minus number of negative ions at each grid point.

(a) The shape of the distribution of ions at $t=0.71 \mathrm{~ns}$ represents an approximate exponential avalanche growth combined with diffusion. The avalanche consists of about $1 \times 10^{6}$ electrons.

(b) At $t=0.81 \mathrm{~ns}$ the shape is different. As the electron cloud propagates, the multiplication and thus the increase of the number of ions is smaller than before. The same effect was found in the 1-D simulations (for example, see Fig. 3.8).

(c) At $1.05 \mathrm{~ns}$ the avalanche has reached the anode. The shape of the ion distribution is very similar to the shape of the electron distribution as shown in image $7.2 \mathrm{~b}$. 

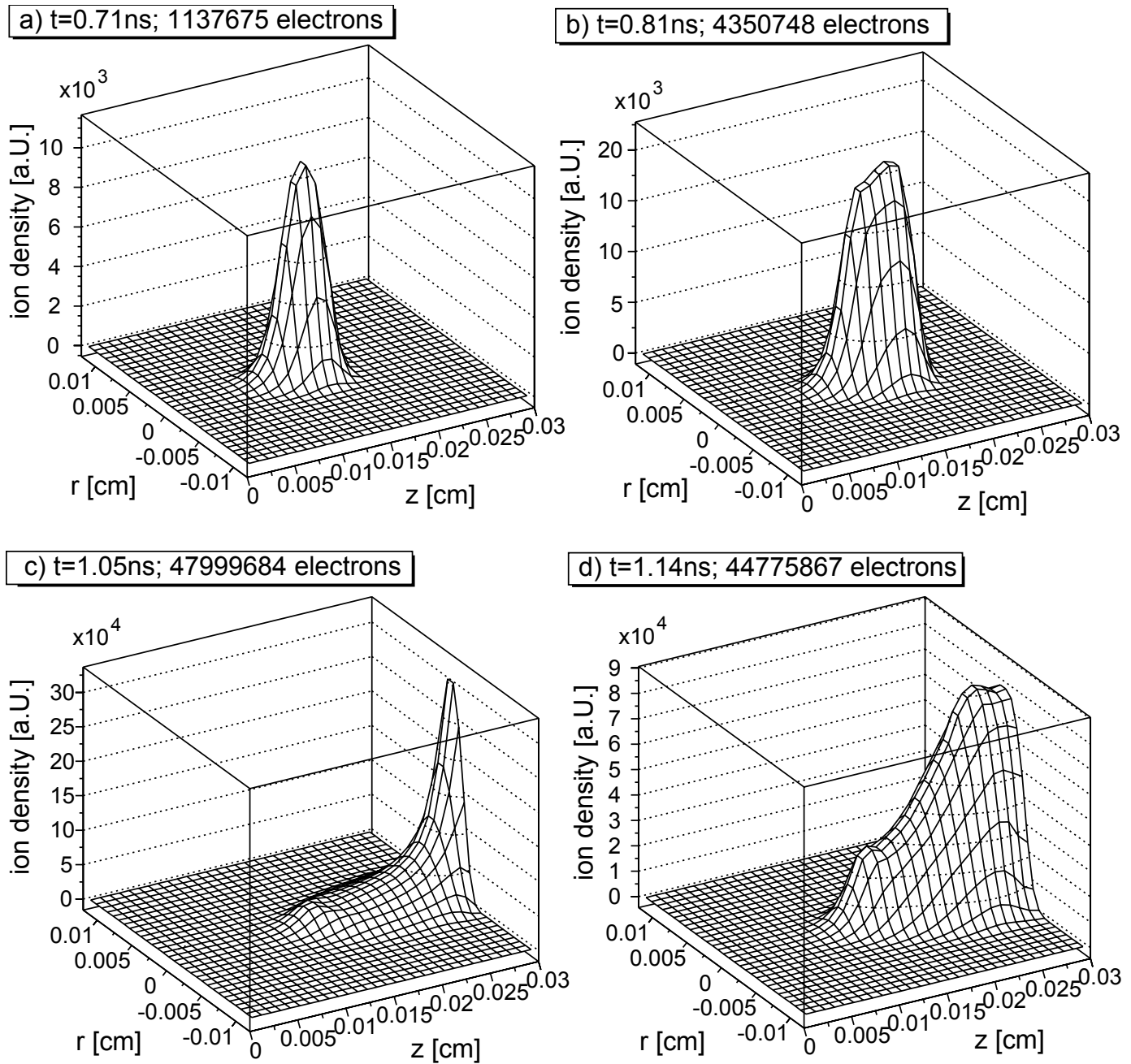

e) t=1.24ns; 16629278 electrons
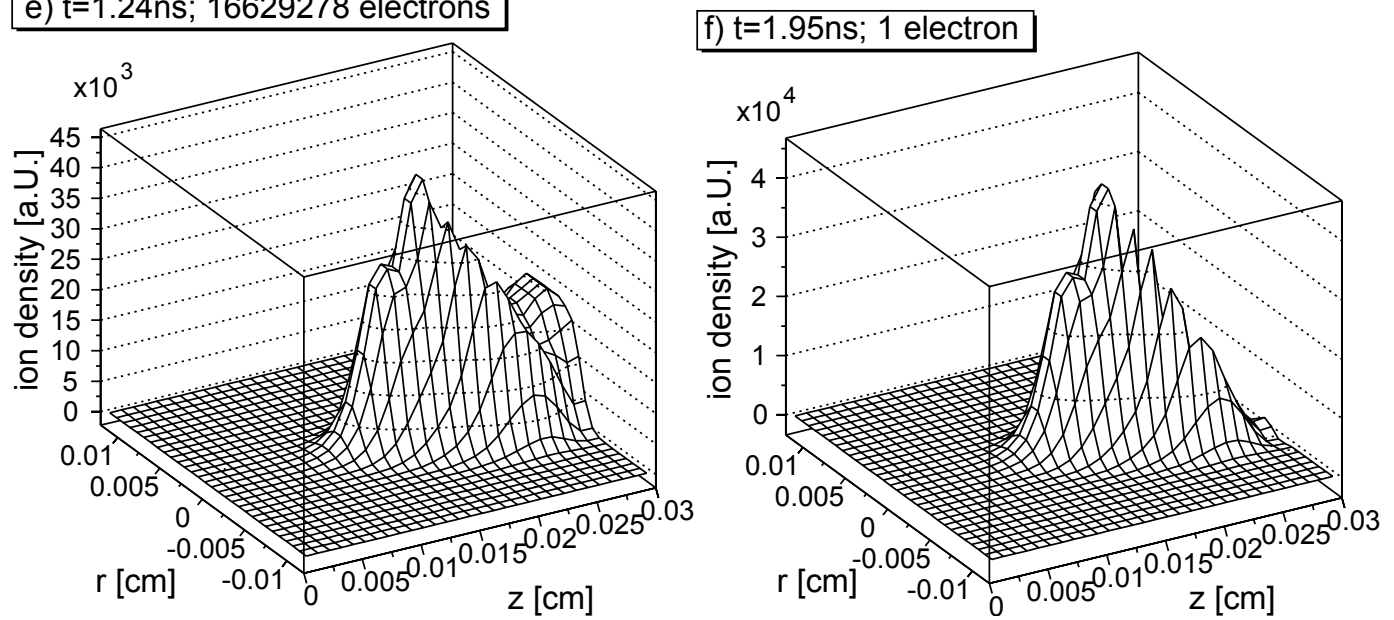

Figure 7.4: Ion density (number of positive ions minus number of negative ions) in an avalanche started by one electron at $(r=0, z=0)$ in a Timing RPC at an operating voltage of $2.8 \mathrm{kV}$. 
(d) At $1.14 \mathrm{~ns}$ the number of positive ions close to the anode decreases rapidly, which is due to the attachment of electrons in that region (compare with Fig. 7.2).

(e) At $1.24 \mathrm{~ns}$ the number of negative ions grows further, leading to a reduction of the density of positive ions.

(f) At $1.95 \mathrm{~ns}$ most electrons either entered the anode or got attached. The density of positive and negative ions close to the anode is similar so that the overall ion density approaches zero here. The same was shown in Fig. 6.6 that was created using the 1.5-D model.

\subsubsection{Total Charge Density}

The combination of the distributions of electrons, positive ions and negative ions leads to a total charge density in the gas gap that is the source of the space charge fields that will be discussed in the following sections. Fig. 7.5 shows the total charge density in an avalanche. Again we simulated an avalanche that was started by a single electron at the position $r=0, z=0$ and at $t=0 \mathrm{~ns}$. Let $n_{e l}(r, z, t), n_{\text {Ipos }}(r, z, t)$ and $n_{\text {Ineg }}(r, z, t)$ be the densities of electrons, positive ions and negative ions, respectively, at the grid point $(r, z)$ and at time $t$, then $n_{q}(r, z, t)=n_{e l}(r, z, t)+n_{\text {Ineg }}(r, z, t)-n_{\text {Ipos }}(r, z, t)$ is the total charge density at that grid point and time. In that sense $n_{q}(r, z, t)$ is the density of negative charge.

(a) At $t=0.76 \mathrm{~ns}$ the avalanche consists of around $2 \times 10^{6}$ electrons. The negative charge of the electrons leads to a negative total charge distribution at the tip of the avalanche. The ions that stay behind lead to a positive total charge distribution at the tail of the avalanche.

(b) At $t=0.86 \mathrm{~ns}$ the avalanche consists of around $7 \times 10^{6}$ electrons.

(c) Just before the first electrons reach the anode (at $t=1.0 \mathrm{ns)} \mathrm{the} \mathrm{avalanche} \mathrm{con-}$ sists of around $2.6 \times 10^{7}$ electrons. While at the tip (tail) of the distribution the density of negative (positive) charge is very high, in the center a region is formed where the total charge density is approximately zero. Here contributions by the positive ions and the negative ions/electrons are balanced.

(d) The first electrons in the center of the avalanche around $r=0$ have entered the anode at around $t=1.05 \mathrm{~ns}$. Here the density of negative charge drops while 'outside' at larger values of $r$ the density of negative charge remains high.

(e) As more electrons enter the anode, the peaks of negative charge density close to the anode decrease. 

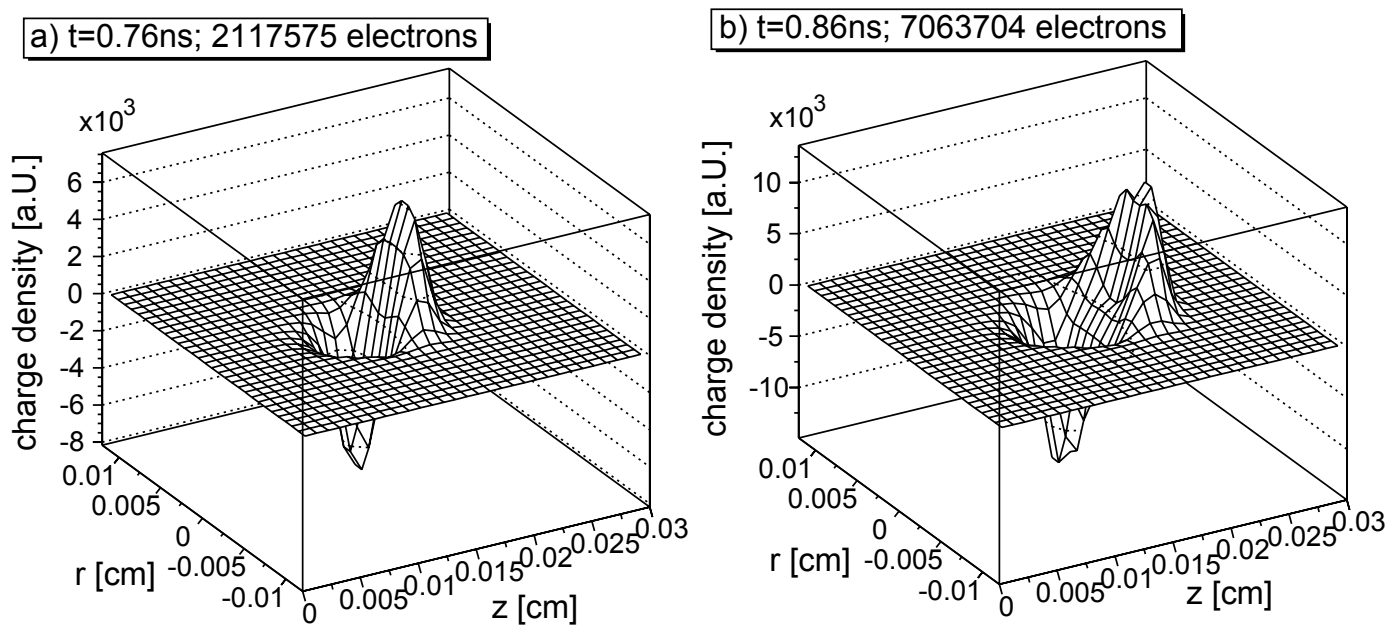

\section{c) t=1.0ns; 26468059 electrons}
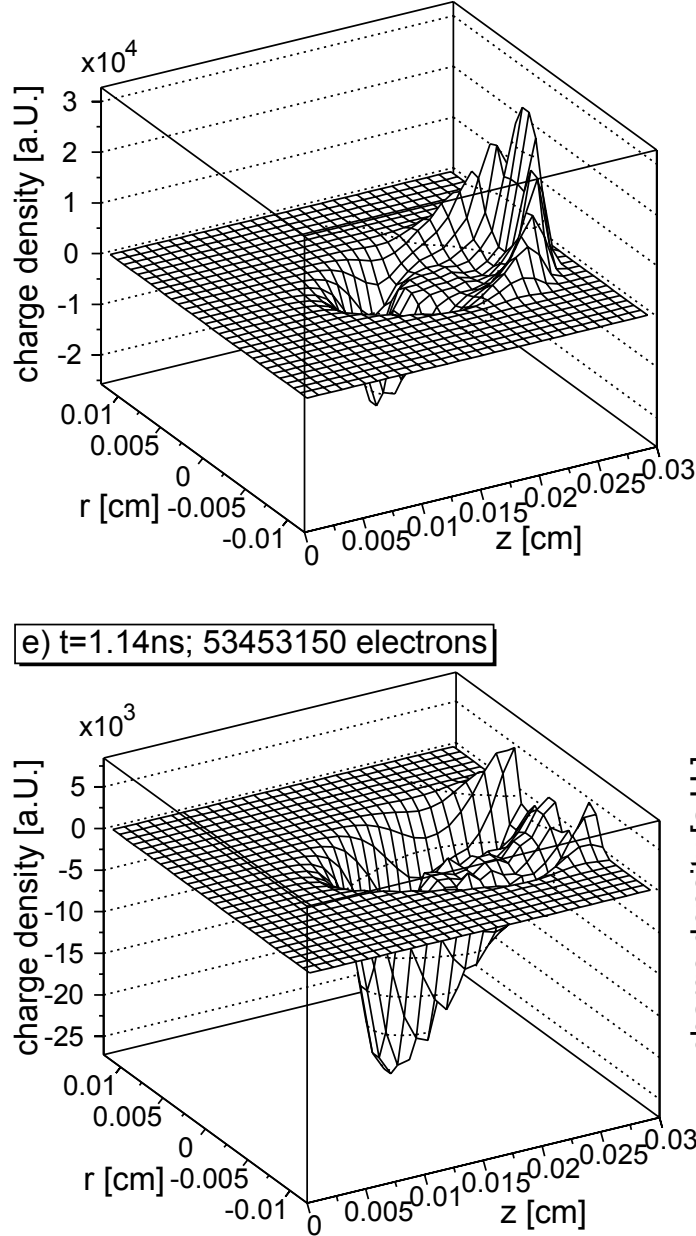

\section{d) t=1.05ns; 45556913 electrons}

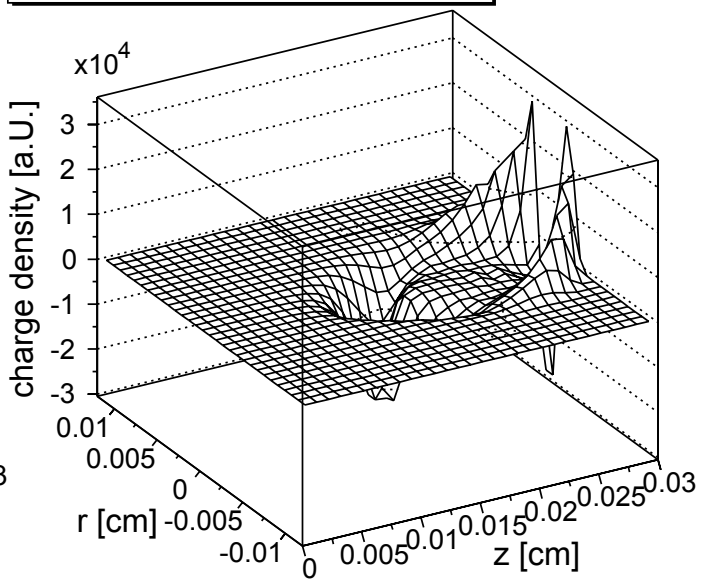

f) t=1.86ns; 223 electrons

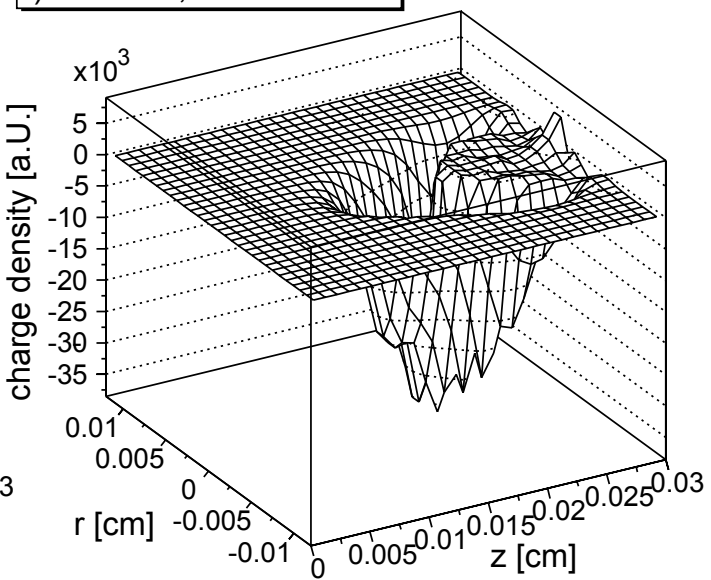

Figure 7.5: Negative charge density (number of electrons plus number of negative ions minus number of positive ions) in an avalanche in a Timing RPC filled with the standard gas mixture at an operating voltage of $2.8 \mathrm{kV}$. The avalanche was started by one electron at $(r=0, z=0)$. 
(f) At $t=1.86 \mathrm{~ns}$ almost all electrons have left the gas gap or got attached. The image now shows the density of negative ions. It is therefore similar to an inverse plot of the one that is shown on Fig. 7.4. Around $r=0$ and $z=0.03$ there is a small peak which is due to the large number of negative ions that is formed in the center of the avalanche. Comparison with Figs. 7.2 and 7.4 shows that here the electrons get attached strongly while at larger values of $r$ more electrons reach the anode.

We have seen that the shapes of the distributions are changing dramatically as the avalanches propagate. The distributions adopt a shape that is very different from one that is only influenced by diffusion. The reason lies in the repulsive and attractive forces between the avalanche charges (the space charge field).

\subsubsection{Electric Field}

The value of the electric field $E(r, z, t)=|\vec{E}(r, z, t)|$ sensed by the electrons in the gas gap determines the values of the gas parameters (Townsend and attachment coefficients, diffusion coefficients and drift velocity). Fig. 7.6 shows the absolute value of $E(r, z, t)$ at different times and at the different grid points given by the $r$ - and $z$ coordinate. Again we simulated an avalanche that was started by a single electron at the position $r=0, z=0$ and at $t=0 \mathrm{~ns}$. We show the value of the the electric field contributed by the avalanche charges (the space charge field). The total field can be calculated by adding the applied external electric field $E_{0}=93.3 \mathrm{kV} / \mathrm{cm}$.

(a) At $t=0.48 \mathrm{~ns}$ the avalanche consists of around 6500 electrons. The field deformations are small. Similar to the previously presented results obtained with the 1-D model (see chapter 5 and Fig. 6.6), the applied electric field is increased at the tip and tail of the electron cloud and decreased in the center. We find exactly the field configuration that was shown schematically in Fig. 1.1.

(b) At $t=0.76 \mathrm{~ns}$ the avalanche consists of around $1.3 \times 10^{6}$ electrons. The maximum field is about 5\% higher than the applied electric field and the minimum field about $15 \%$ lower.

(c) At $t=0.95 \mathrm{~ns}$ the avalanche is ten times as large (around $1.3 \times 10^{7}$ electrons). At the maximum (minimum) the field is increased (decreased) by about $30 \%$ as compared to the applied electric field.

(d) As the first electrons reach the anode (at $t=1.05 \mathrm{ns)} \mathrm{the} \mathrm{avalanche} \mathrm{consists} \mathrm{of}$ about $3.3 \times 10^{7}$ electrons and the maximum space charge field is of the same order of magnitude as the applied electric field. At the minimum it is about $40 \%$ lower. 

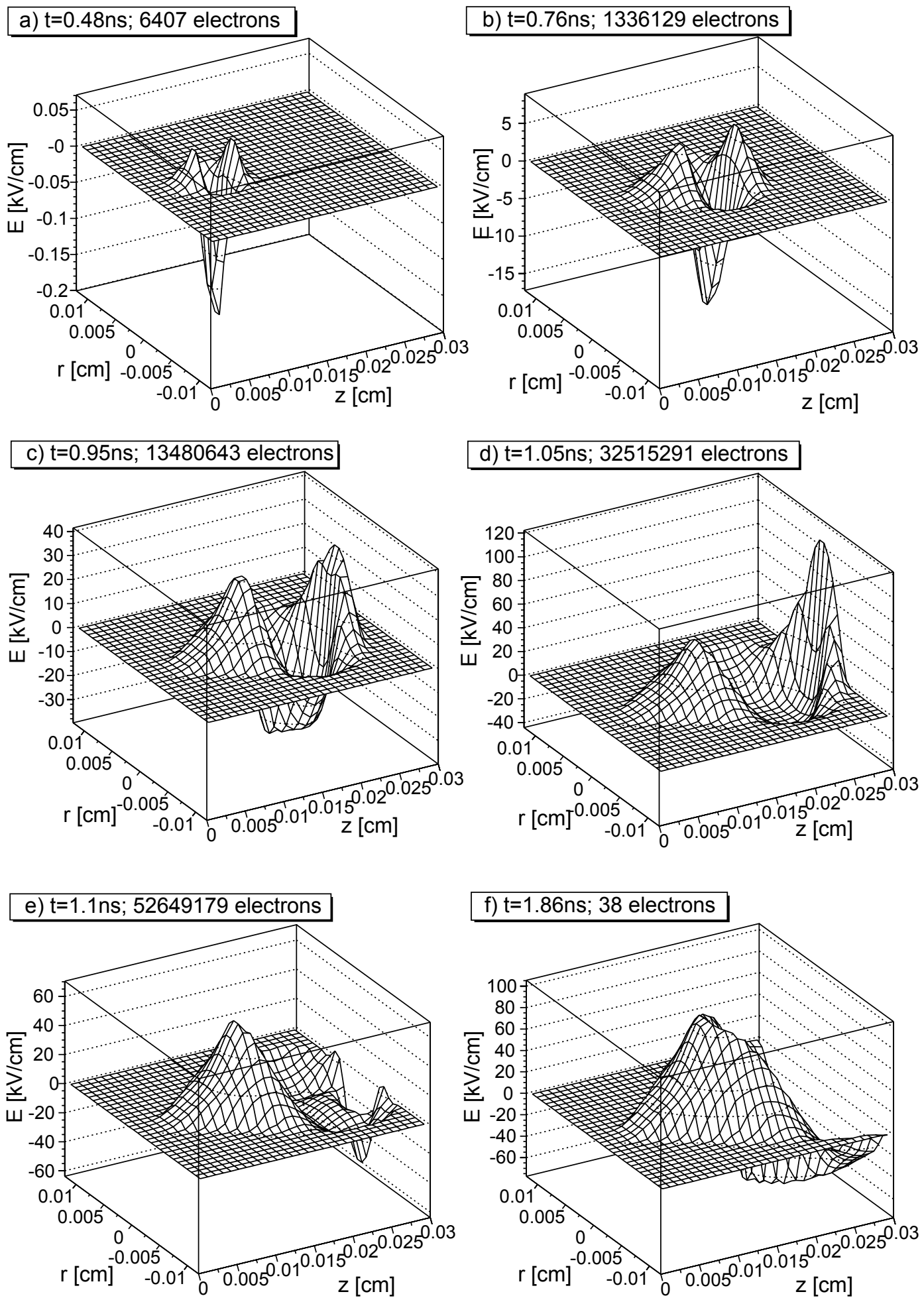

Figure 7.6: Absolute value of the electric field $E(r, z, t)$ that is contributed by the space charge in an avalanche started by one electron at $(r=0, z=0)$ in a $0.3 \mathrm{~mm}$ gap Timing RPC at an operating voltage of $2.8 \mathrm{kV}$. The applied electric field $E_{0}=93.3 \mathrm{kV} / \mathrm{cm}$ is not included. 

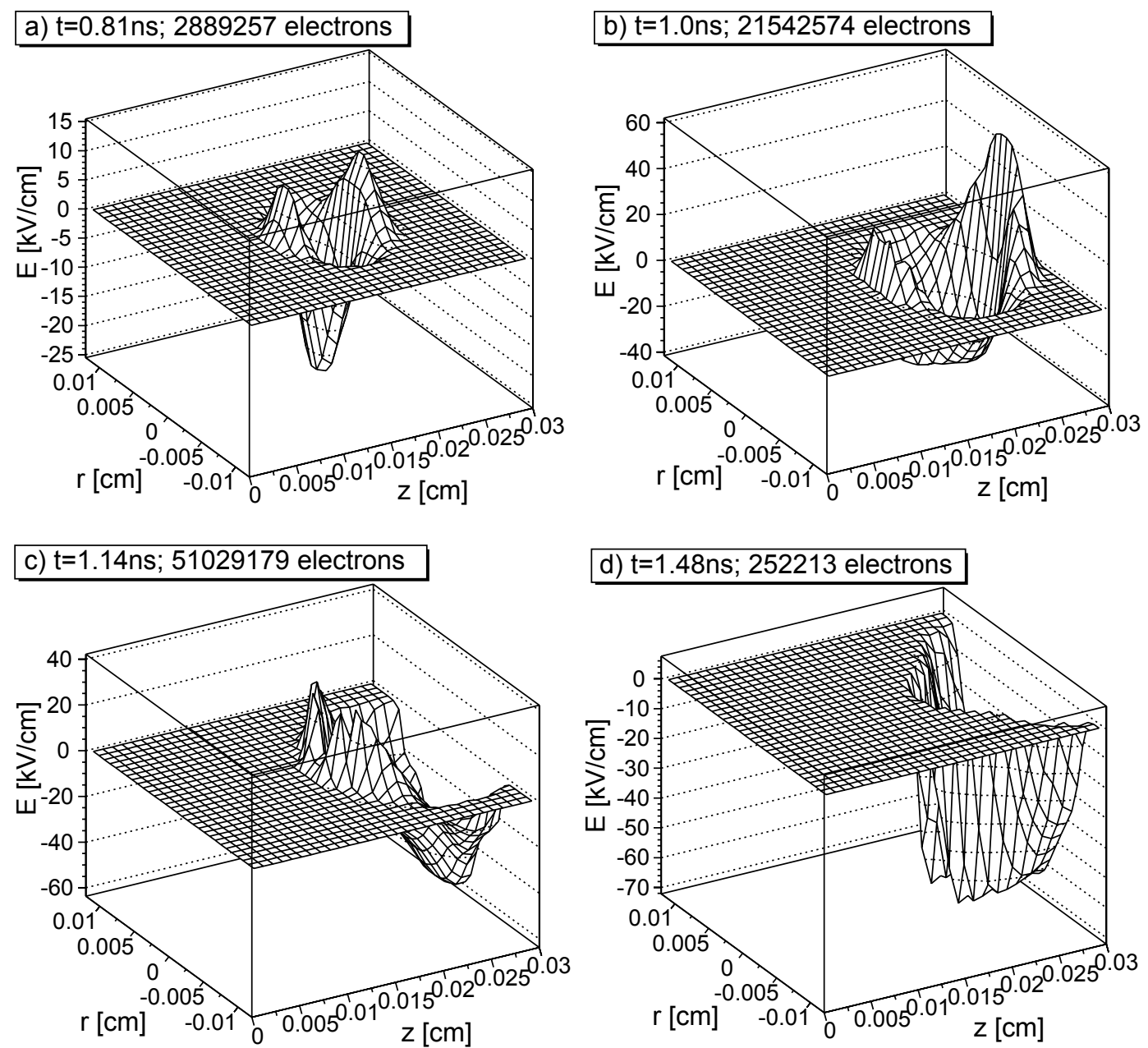

Figure 7.7: Absolute value of the space charge field. Contrary to Fig. 7.6 the field is set zero everywhere where no electrons are situated.

(e) At $t=1.1 \mathrm{~ns}$ the electric field drops dramatically in front of the anode. A large number of electrons have already left the gas gap. Still the multiplication in other regions is large: The avalanche has around $5.3 \times 10^{7}$ electrons. At the maximum the field is almost $60 \%$ higher than the applied field.

(f) At $t=1.86 \mathrm{~ns}$ almost all electrons have left the gas gap or got attached. We find that in a large region in front of the anode the field is lowered by up to $60 \%$. The field deformations are due to the ions that remain in the gas gap.

From this data we learn that there are regions of drastically decreased electric field strength in an avalanche that are the cause of the saturated growth and the low observed final avalanche charges. We also observe regions where the electric field is increased dramatically, which raises the question if the increased multiplication in those regions 
do compensate the decreased multiplication in the center. An answer can be given if the value of the field that is sensed by the electrons in the gas gap is investigated. In Fig. 7.7 we show the value of the electric field only at the positions in the gas gap, where we find electrons.

$(a, b)$ In the initial phase of the avalanche, where the electron cloud has not yet reached the anode (at $t=0.81 \mathrm{~ns}$ and at $t=1.0 \mathrm{~ns}$ ), the electrons are distributed both in regions with increased and lowered electric field strength. While at the tip, where the field can be increased dramatically, the electron density is large (compare to Fig. 7.2 a), at the tail where the field is also increased there are only a few electrons.

(c) At a later stage (at $t=1.14 \mathrm{ns),} \mathrm{where} \mathrm{the} \mathrm{avalanche} \mathrm{has} \mathrm{reached} \mathrm{the} \mathrm{anode} \mathrm{and}$ consists of about $5.1 \times 10^{7}$ electrons, the field is lowered almost everywhere, where we find electrons. But still we find a region at the tail of the electron cloud, where electrons sense an electric field strength that is increased by up to $40 \%$.

(d) The electrons then all enter the region of decreased electric field strength in front of the anode. At $t=1.48 \mathrm{~ns}$, where the avalanche has $5.1 \times 10^{5}$ electrons, and at all later stages, the electrons will sense an accordingly lowered drift velocity and effective Townsend coefficient.

We summarize that there only a minority of electrons is situated in the regions where the field is increased by the space charge effect. The increased multiplication at the regions of higher field strength is more than compensated by the dramatically decreased multiplication in the center of the electron cloud, where most of the electrons are situated.

\subsubsection{Drift Velocity}

The value of the electric field $E(r, z, t)$ sensed by the electrons in the gas gap determines the value of the drift velocity $v_{D}(r, z, t)$ of the electrons. Fig. 7.8 shows the value of $v_{D}(r, z, t)$ at different times and at the different grid points given by the $r$ and $z$-coordinate. Again we simulated an avalanche that was started by a single electron at the position $r=0, z=0$ and at $t=0 \mathrm{~ns}$. In Fig. $7.8 v_{D}(r, z, t)$ is set zero at all positions where no electrons are situated.

(a) At $t=0.76 \mathrm{~ns}$ the avalanche consists of around $2.3 \times 10^{6}$ electrons. The deformations in the distribution of the drift velocity in the gas gap are up to $10 \%$.

(b) At $t=0.95 \mathrm{~ns}$ the drift velocity is about $25 \%$ larger at the tip of the electron distribution, around 10\% higher at the tail and around 25\% lower in the center where the largest part of the electrons is situated. 

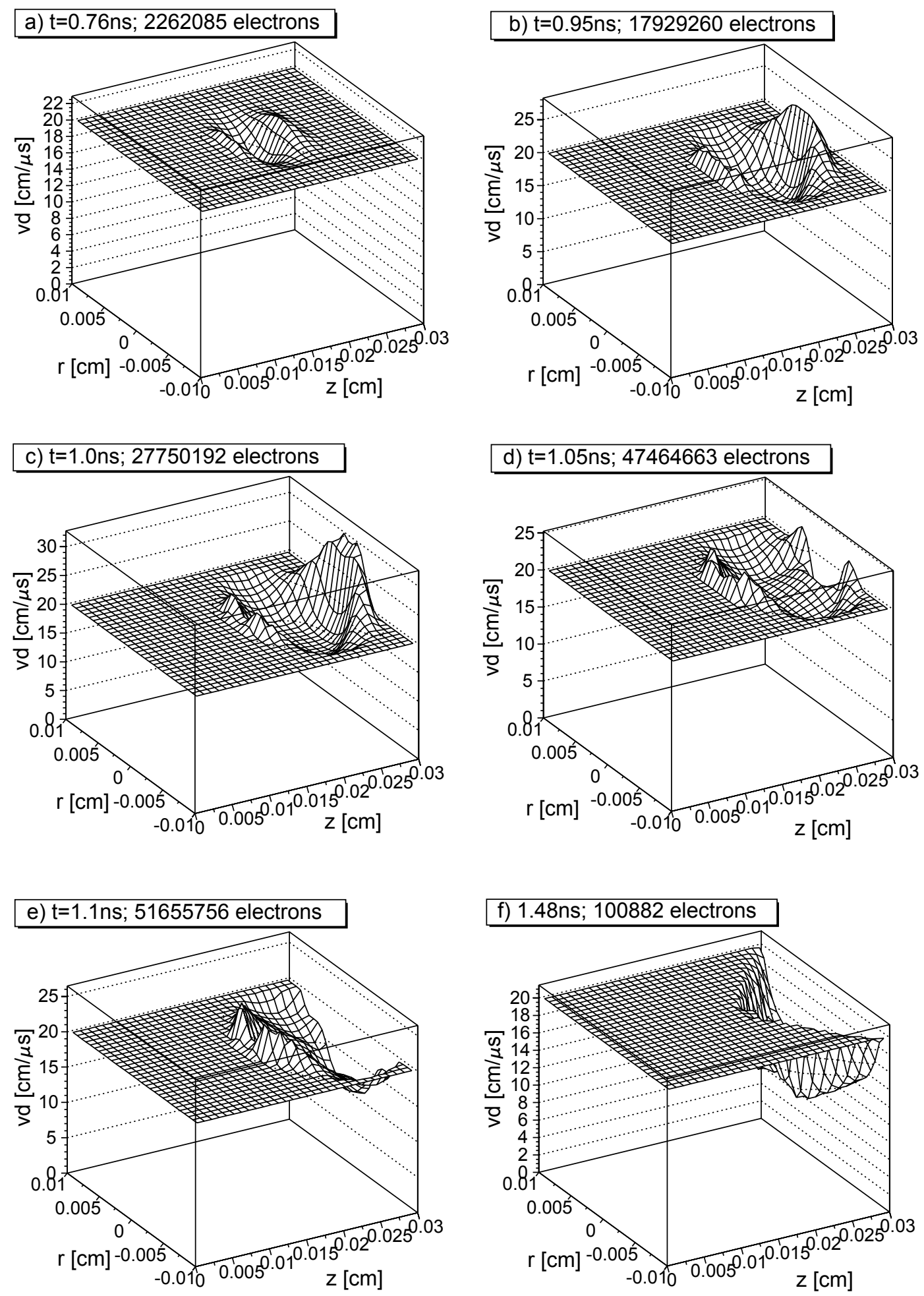

Figure 7.8: Drift velocity sensed by the electrons in the gas gap. The drift velocity is set zero everywhere where no electrons are situated. 
(c) At $t=1.0 \mathrm{~ns}$ the first electrons reach the anode surface at the tip of the avalanche, where the drift velocity is now increased by $50 \%$. The avalanche consists of $2.3 \times 10^{7}$ electrons.

(d) As the total electric field drops, also the drift velocity decreases in the region in front of the anode.

(e) At $t=1.1 \mathrm{~ns}$ almost all electrons are in a region with decreased drift velocity. Only at the tail the drift velocity is around $25 \%$ increased.

(f) At $t=1.48 \mathrm{~ns}$ there are around $1.0 \times 10^{5}$ electrons left in the gas gap. The drift velocity is lowered for all those electrons. At the minimum it is only half the value of the drift velocity at the applied electric field strength.

\subsubsection{Effective Townsend Coefficient}

In this section we investigate the value of the parameter that determins the avalanche multiplication during the evolution of an avalanche in the gas gap of a Timing RPC: the effective Townsend coefficient $\alpha_{\text {eff }}(r, z, t)=\alpha(E(r, z, t))-\eta(E(r, z, t))$. Fig. 7.9 shows the value of $\alpha_{e f f}(r, z, t)$ at different times and at the different grid points given by the $r$-and $z$-coordinate. Again we simulated an avalanche that was started by a single electron at the position $r=0, z=0$ and at $t=0 \mathrm{~ns}$. A positive effective Townsend coefficient means that on average the number of electrons in the corresponding grid point will increase. A negative effective Townsend coefficient means that on average the number of electrons will decrease. The value of $\alpha_{e f f}(r, z, t)$ at the applied electric field strength $E_{0}$ we call $\alpha_{0}$.

(a) At $t=0.95 \mathrm{~ns}$ the avalanche has almost reached the anode and consists of around $1.9 \times 10^{7}$ electrons. The deformations in the distribution of the effective Townsend coefficient in the gas gap are very large. At the tip (tail) of the electron cloud the increase is more than $100 \%(50 \%)$ as compared to $\alpha_{0}$, in the center the effective Townsend coefficient is below zero.

(b) At $t=1.0 \mathrm{~ns}$ the first electrons have reached the anode. We find that now the effective Townsend coefficient reaches a value of three times $\alpha_{0}$. In the center of the electron distribution, where most of the $3.0 \times 10^{7}$ electrons are situated, the value of $\alpha_{\text {eff }}(r, z, t)$ is below zero.

(c) At $t=1.1 \mathrm{~ns}$ the avalanche has grown to about $6.1 \times 10^{7}$ electrons. Up to now at most positions the value of $\alpha_{e f f}(r, z, t)$ was still positive. Now we find a region in front of the anode where $\alpha_{e f f}(r=0, z \rightarrow g, t)<-1000 / \mathrm{cm}$.

(d) At $t=1.24 \mathrm{~ns}$ most electrons are situated in the region of very low effective Townsend coefficient in front of the anode. In the largest fraction of this region 

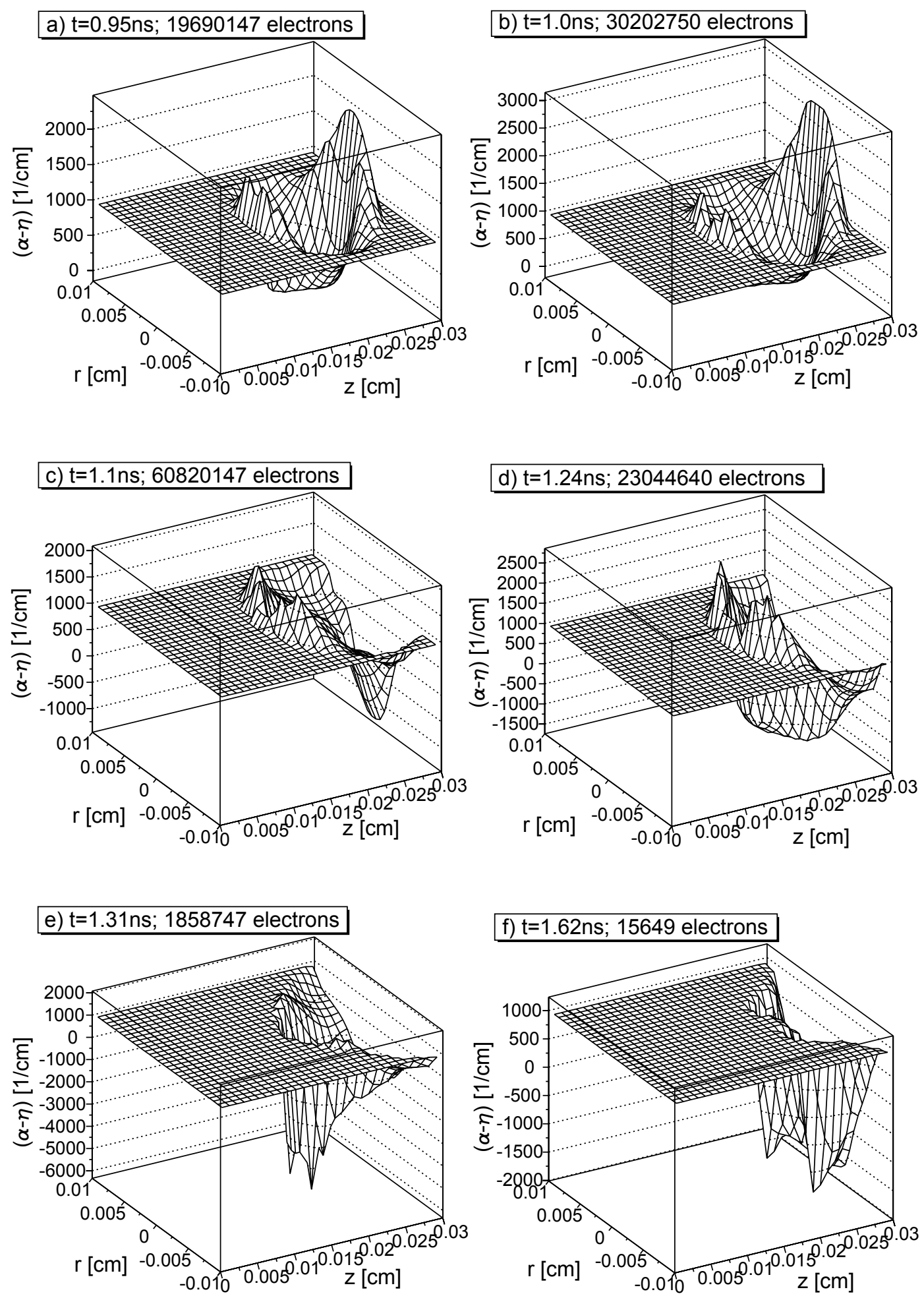

Figure 7.9: Effective Townsend coefficient at the positions where electrons are situated in the gas gap. 
the value of $\alpha_{e f f}(r, z, t)$ is negative. The minimum value is around $-1500 / \mathrm{cm}$. Accordingly, the number of electrons has dropped to $2.3 \times 10^{7}$. However, in the region further away from the anode, we still find a region of strong multiplication with an effective Townsend coefficient up to 2.5 times as large as $\alpha_{0}$. As was mentioned earlier, in this region the electron density is small so that the effect on the avalanche growth at this stage is small.

(e) At $t=1.31 \mathrm{~ns}$ the number of electrons has dropped to $1.9 \times 10^{6}$ electrons. Almost everywhere the effective Townsend coefficient is much lower than $\alpha_{0}$. The minimum value reaches $-6000 / \mathrm{cm}$ !

(f) At $t=1.62 \mathrm{~ns}$ the few electrons left in the gas gap undergo strong attachment. The minimum value of $\alpha_{e f f}(r, z, t)$ is around $-2000 / \mathrm{cm}$.

\subsubsection{Longitudinal Electric Field}

The value of the $z$-component of the electric field $E_{z}(r, z, t)$ that is sensed by the electrons in the gas gap is the parameter that determines the velocity with that the electrons in the avalanches reach the anode. Also this value can directly be compared to the values that were obtained using the 1.5-D model. Fig. 7.10 shows the value of $E_{z}(r, z, t)$ at different times at the different grid points given by the $r$-and $z$-coordinates. Again we simulated an avalanche that was started by a single electron at the position $r=0$, $z=0$ and at $t=0 \mathrm{~ns}$. We show the value of the space charge field. The total longitudinal field can be calculated from that by adding the applied external electric field $E_{0}=93.3 \mathrm{kV} / \mathrm{cm}$.

(a) At $t=0.57 \mathrm{~ns}$ the avalanche consists of around $1.7 \times 10^{5}$ electrons. The field deformations are small. The field is increased at the tip and tail of the electron cloud and decreased at its tail.

(b) At $t=0.95 \mathrm{~ns}$ the avalanche consists of around $2.7 \times 10^{7}$ electrons. At the maximum the longitudinal field is almost $50 \%$ higher than the applied electric field and at the minimum it is about $40 \%$ lower.

(c) At $t=1.0 \mathrm{~ns}$ the avalanche has grown to around $4.1 \times 10^{7}$ electrons and the first electrons reach the anode. The maximum longitudinal space charge field is about the same order of magnitude as the applied electric field. At the minimum it is about $40 \%$ lower.

(d) At $t=1.05 \mathrm{~ns}$ the longitudinal electric field drops dramatically in front of the anode. At the minimum it is almost $60 \%$ lower than the applied electric field. The avalanche has $6.7 \times 10^{7}$ electrons. 
a) t=0.57ns; 170674 electrons

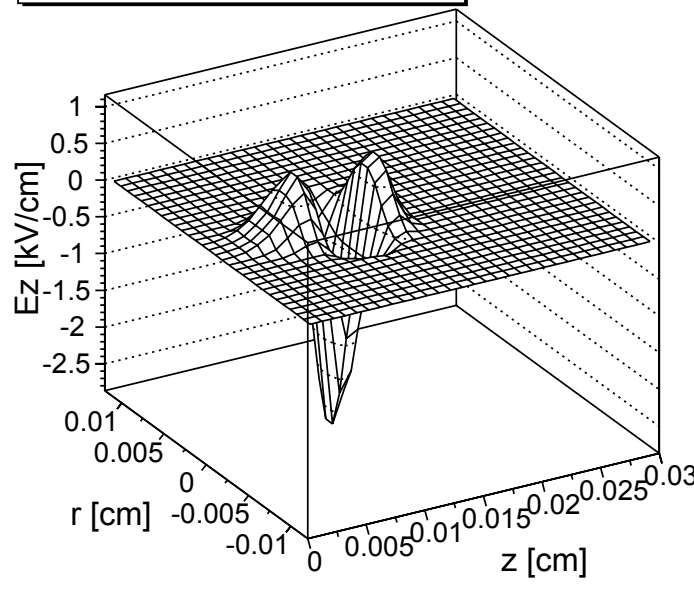

\section{c) t=1.0ns; 40761458 electrons}

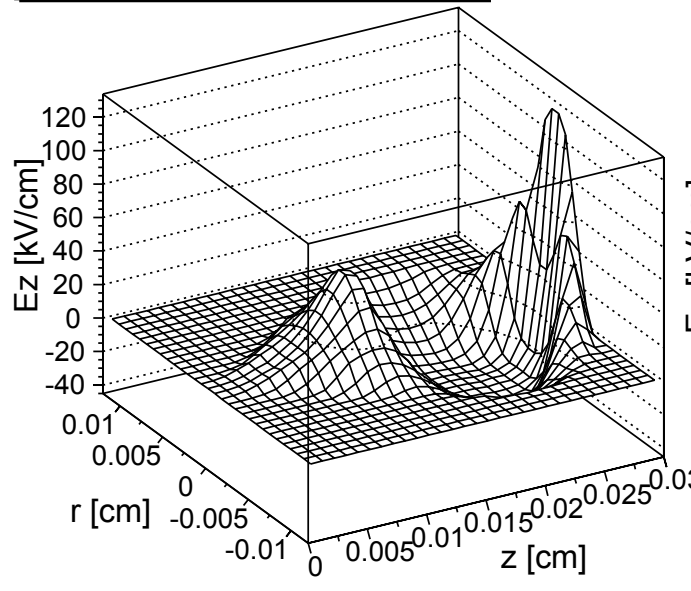

e) $t=1.14 n s ; 56189097$ electrons

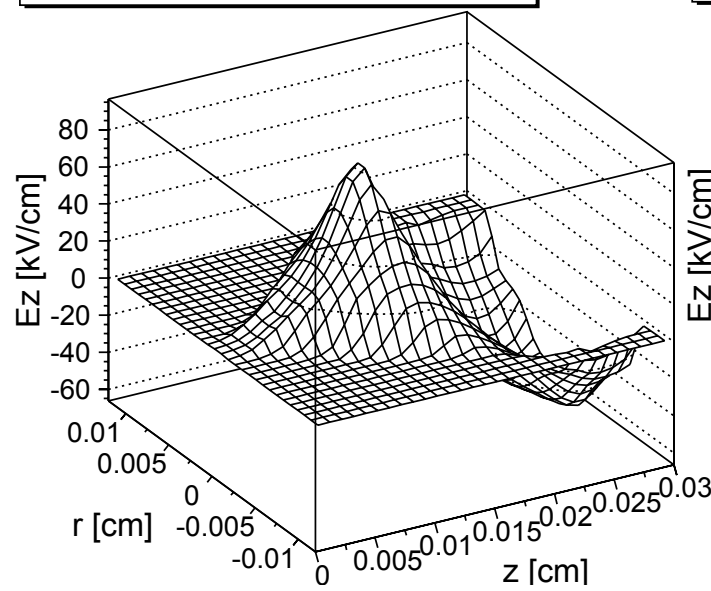

\section{b) t=0.95ns; 26465899 electrons}

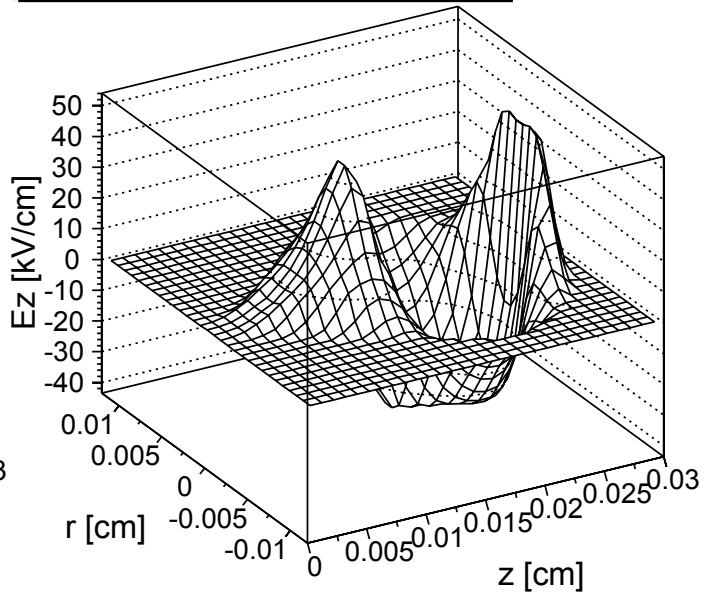

d) t=1.05ns; 66992735 electrons
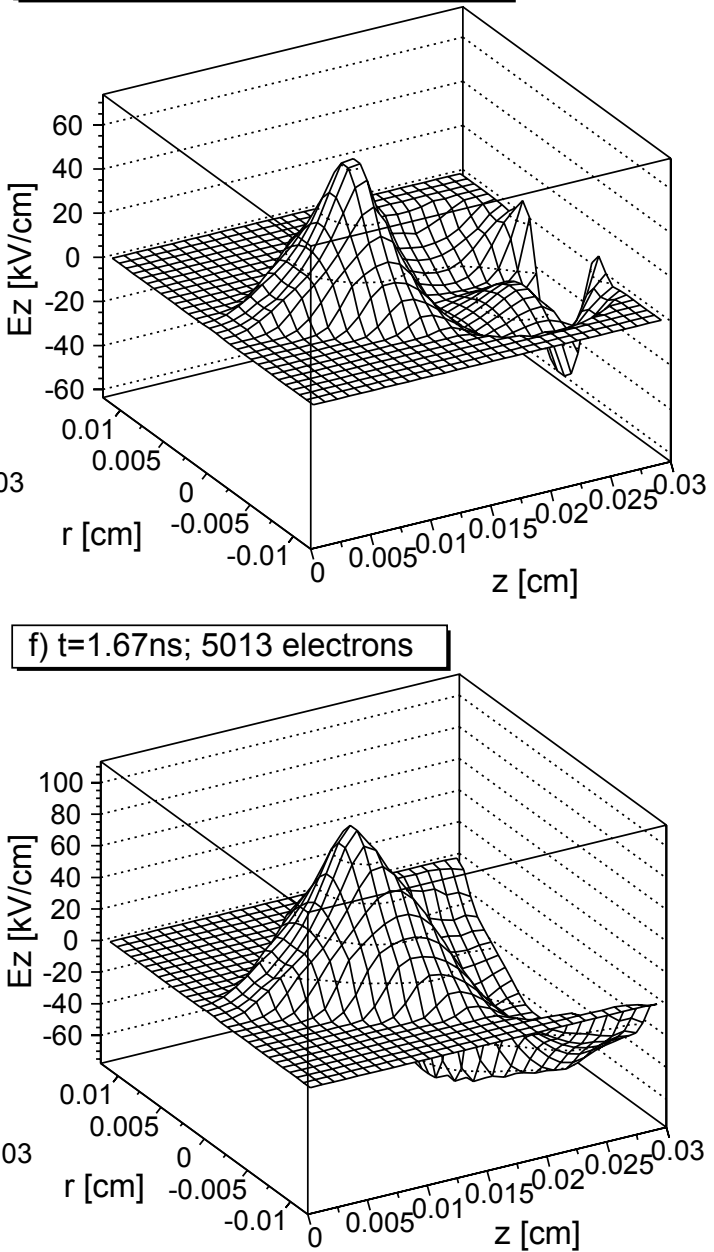

Figure 7.10: $z$-component of the space charge field $E_{z}(r, z, t)$ in an avalanche in a $0.3 \mathrm{~mm}$ gap Timing RPC filled with the standard gas mixture and with an operating voltage of $2.8 \mathrm{kV}$. The avalanche was started by one electron at $(r=0, z=0)$. 
(e,f) At $t>1.1 \mathrm{~ns}$ the region of decreased longitudinal field in front of the anode grows. At the minimum the field is about $60 \%$ lower. At the final stage all free electrons have either left the gas gap or got attached; the field deformations are now only due to the ions.

The longitudinal field behaves similarly to the total electric field. Again we also investigate this parameter at the positions where electrons are actually situated. In Fig. 7.11 the longitudinal electric field is set zero at all other positions in the gas gap.

(a) In the initial phase of the avalanche (at $t=0.81 \mathrm{~ns}$ ), where the electron cloud has not yet reached the anode, the electrons are distributed both in regions with increased and lowered longitudinal electric field. If compared to Fig. 7.2 a we see that at the tip, where the field is increased dramatically, the electron density is large while at the tail, where the field is also increased, there are only a few electrons. Accordingly, the $z$-component of the electron drift velocity will be increased at the tip and the tail of the electron cloud while it is decreased in the large region at the center.

(b) At $t=1.0 \mathrm{ns,} \mathrm{where} \mathrm{the} \mathrm{avalanche} \mathrm{has} 3.9 \times 10^{7}$ electrons, the first electrons reach the anode. The maximum longitudinal field is increased dramatically at the tip of the electron distribution. At the tail, where there are not a lot of electrons, it is also increased. At the center of the electron cloud the longitudinal field is decreased in a large region.

(c,d) At later stages the longitudinal field is lowered almost everywhere, where we find electrons. At the tail of the electron cloud there is a region where the longitudinal electric field sensed by the electrons is increased by around $10 \%$.

(e) The electrons then all enter the region of decreased longitudinal electric field in front of the anode.

(f) As was observed in Fig. 7.2f the electrons disappear fastest in the center of the avalanche around $r=0$. At $t=1.67 \mathrm{~ns}$ we find electrons only at the regions further 'outside' at larger values of $r$. Here the field is lowered by up to $60 \%$.

The development of the longitudinal electric field is very similar to what we obtained with the 1.5-D model (for example, see Fig. 6.6).

\subsubsection{Radial Electric Field}

The value of the $r$-component of the electric field $E_{r}(r, z, t)$ that is sensed by the electrons in the gas gap is the parameter that determines the radial spread of the electron cloud on top of the transverse diffusion. Fig. 7.12 shows the value of $E_{r}(r, z, t)$ at 

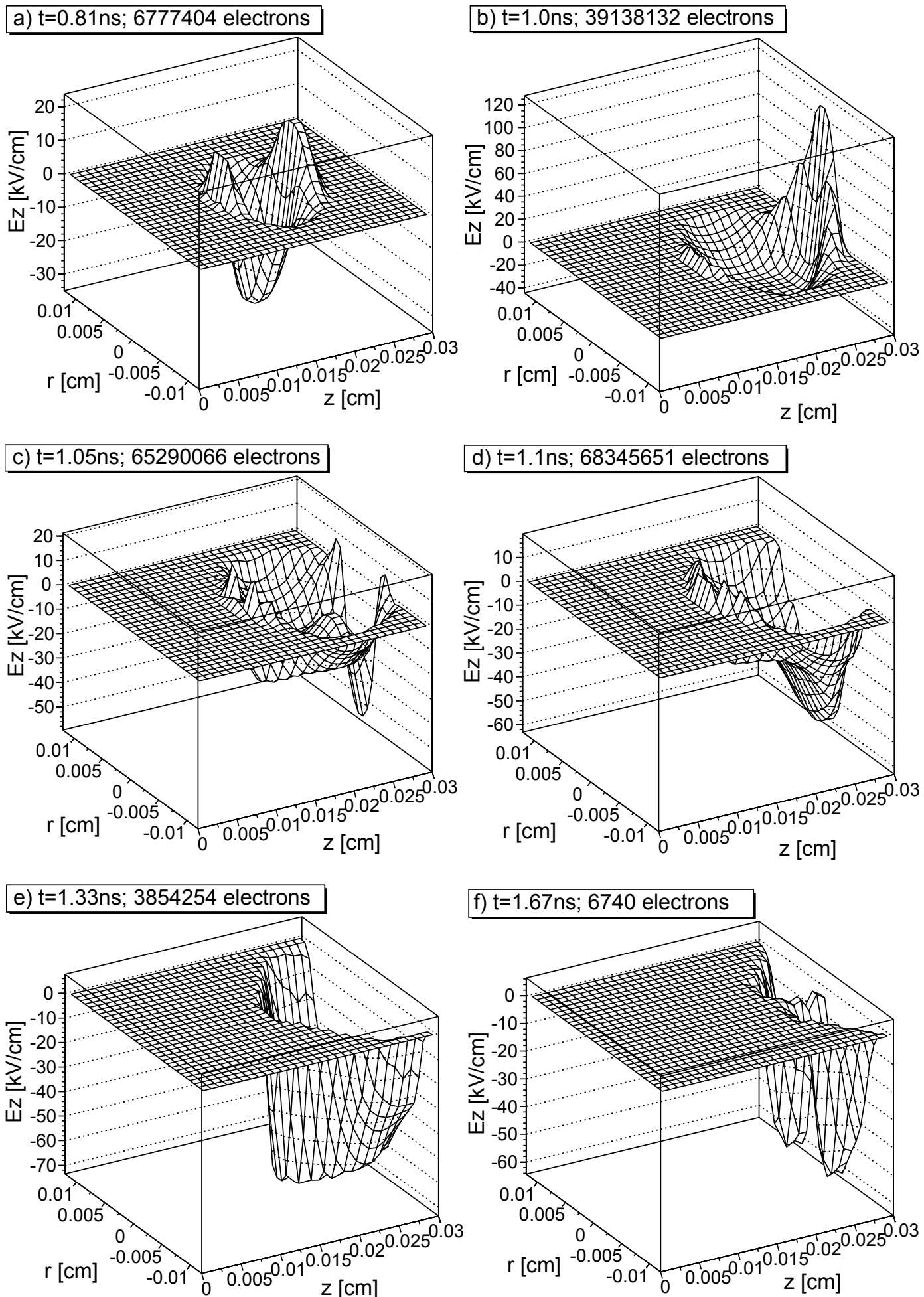

Figure 7.11: $z$-component of the space charge field. The field is calculated only at the points where electrons are situated. 
different time steps and at the different grid points given by the $r$ - and $z$-coordinates. Again we simulated an avalanche that was started by a single electron at the position $r=0, z=0$ and at $t=0 \mathrm{~ns}$.

(a) At $t=0.81 \mathrm{~ns}$ the avalanche consists of around $4.4 \times 10^{6}$ electrons. The radial component of the electric field has values of up to $10 \%$ of the applied longitudinal electric field. A positive value means that electrons that sense this field will be accelerated towards larger values of $r$. As expected from the total charge distribution encountered in the avalanches (see Fig. 7.5), the radial field repels electrons from the center of the avalanche at $r=0$ at the tip of the avalanche, while it attracts electrons towards the center at the tail. Since at the tip of the avalanche the electron density is largest, the radial space charge effect is a very important effect.

(b) At $t=0.95 \mathrm{~ns}$ the maximum radial field reaches about $40 \%$ of the applied electric field.

(c) At $t=1.0 \mathrm{~ns}$ the avalanche has grown to around $3.4 \times 10^{7}$ electrons. The first electrons reach the anode. The maximum radial space charge field is about $50 \%$ of the applied electric field.

(d) At $t=1.05 \mathrm{~ns}$ the distribution of the radial electric field strength starts to change in the region in front of the anode $(z \rightarrow 0.03 \mathrm{~cm})$. Here the maximum radial space charge field reaches values of about $20 \%$ of the applied electric field, while in the center of the gas gap it it is about $60 \%$ of the applied electric field.

(e) At $t=1.14 \mathrm{~ns}$ value of the radial field in front of the anode has decreased. The radial field now attracts electrons to the center everywhere.

(f) At $t=1.52 \mathrm{~ns}$ there are only $1.8 \times 10^{5}$ free electrons left in the gas gap. The value of the radial space charge field is around zero at $z \rightarrow 0.03 \mathrm{~cm}$. Elsewhere the maximum can exceed the applied longitudinal electric field.

Again we show a plot, where the radial electric field is calculated only at positions where we find electrons (Fig. 7.13) in order to view more clearly the radial field that is sensed by the electrons.

(a) $t=0.81 \mathrm{~ns}$ : At the early stages of the avalanches, where no electrons have yet reached the anode, the electrons are attracted (repelled) to (from) the center of the avalanche at $r=0$ at the tip (tail) of the avalanche, as we stated previously.

(b) $t=1.0 \mathrm{~ns}$ : The first electrons reach the anode.

(c) $t=1.1 \mathrm{~ns}$ : As many free electrons have left the gas gap, the density of positive ions now is larger than the density of electrons. As mentioned previously, the field now attracts electrons to the center everywhere. 
a) $\mathrm{t}=0.81 \mathrm{~ns} ; 4359134$ electrons

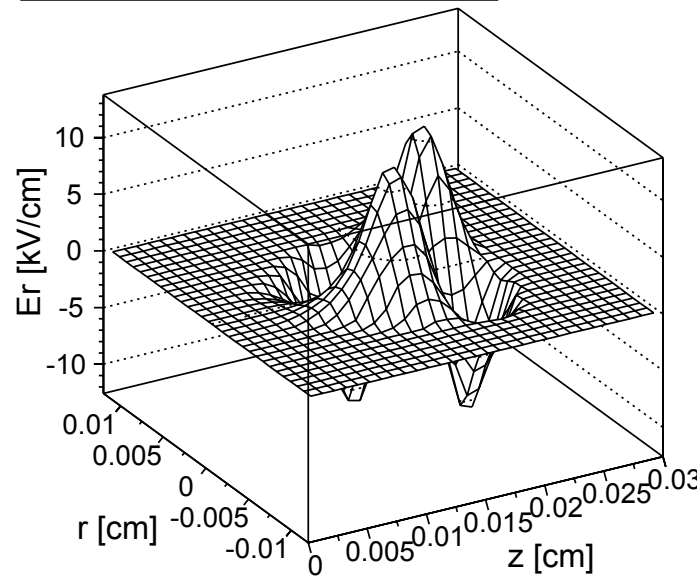

c) t=1.0ns; 33912307 electrons

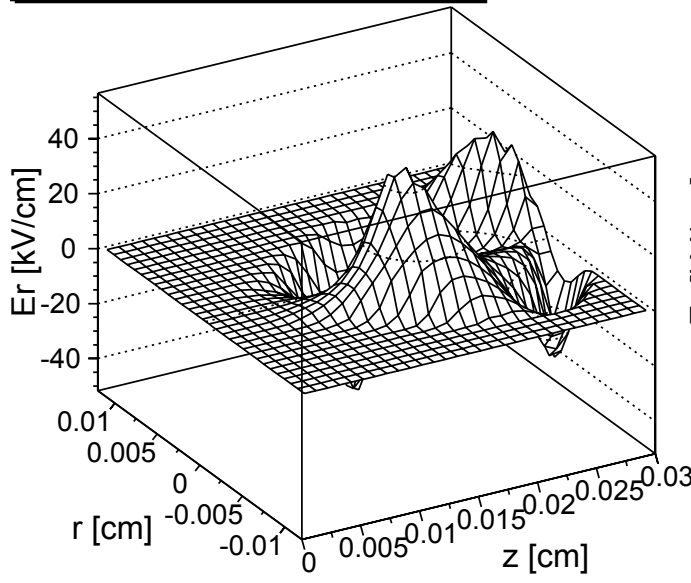

e) $t=1.14 n s ; 56207098$ electrons

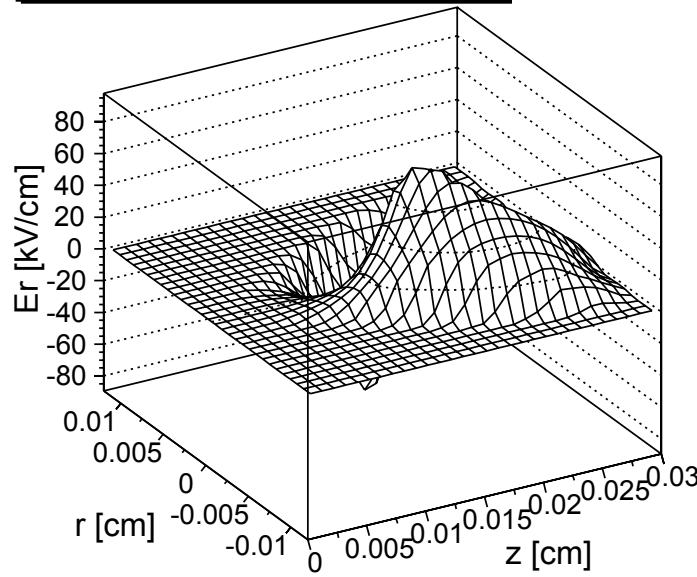

\section{b) t=0.95ns; 21399807 electrons}

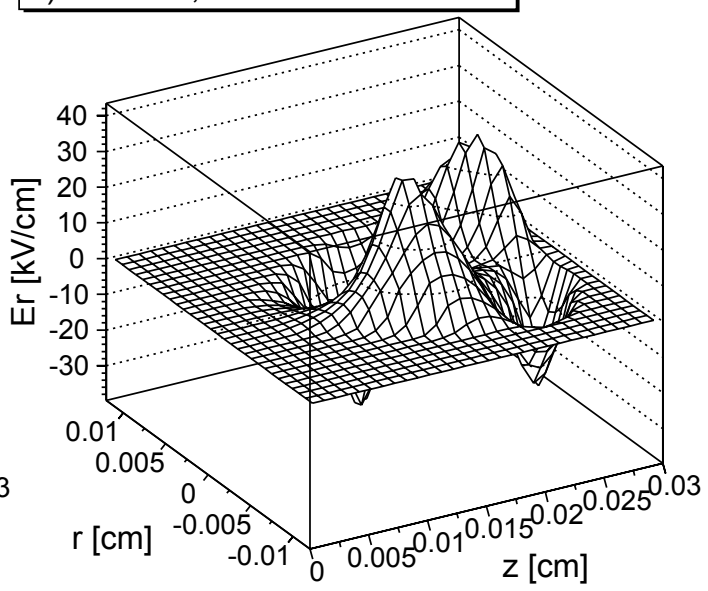

\section{d) t=1.05ns; 57256845 electrons}
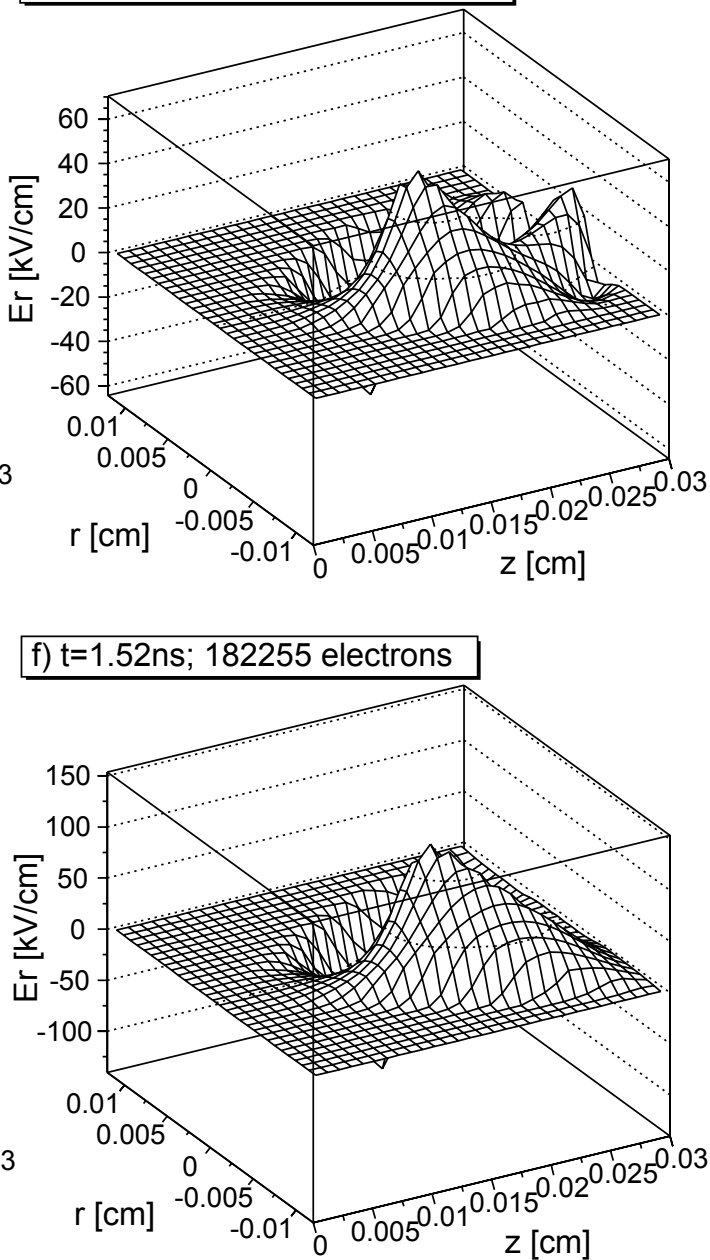

Figure 7.12: $r$-component of the space charge field $E_{r}(r, z, t)$ in an avalanche started by one electron at $(r=0, z=0)$ in a Timing RPC filled with the standard gas mixture and with an operating voltage of $2.8 \mathrm{kV}$. 

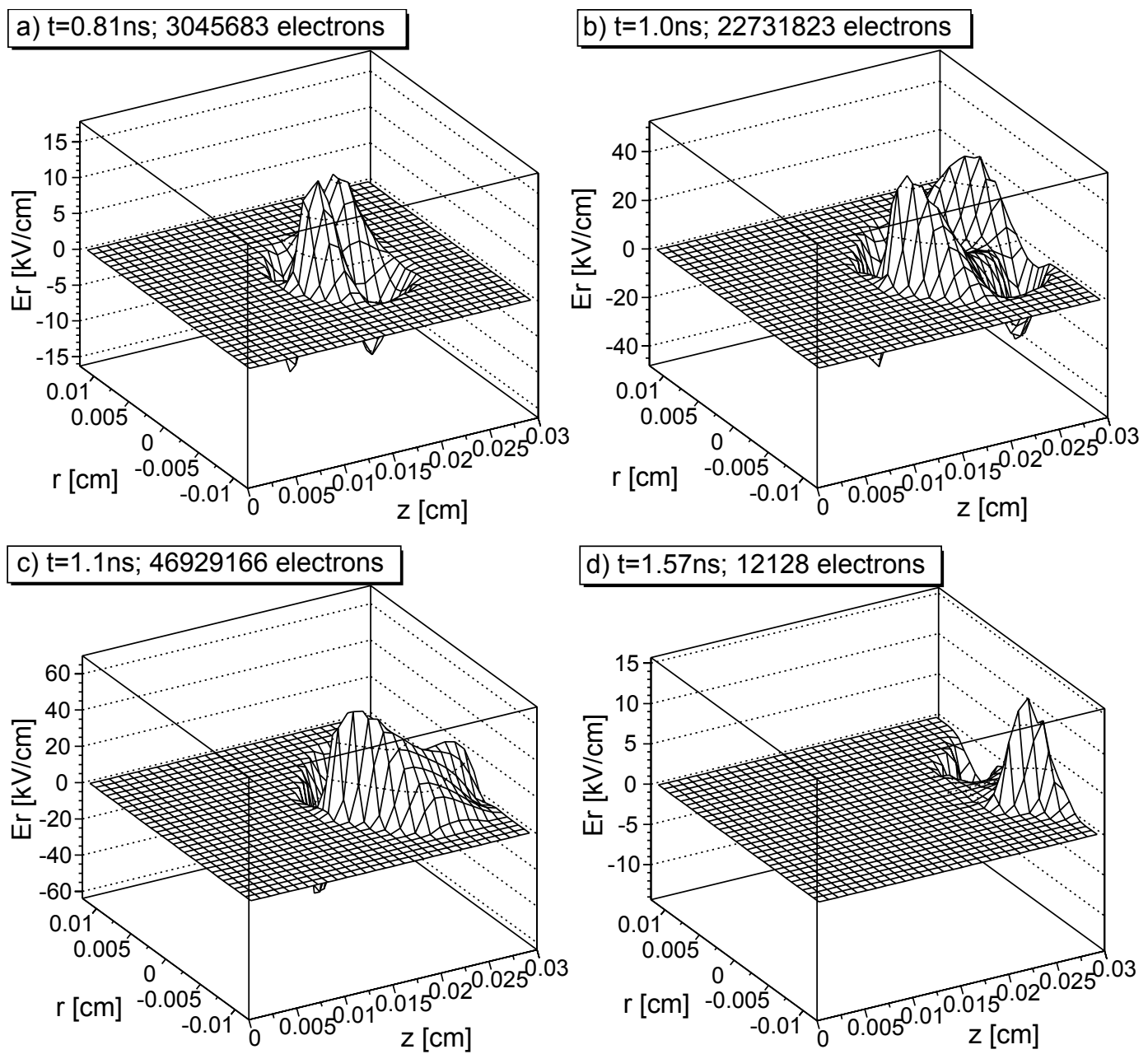

Figure 7.13: $r$-component of the space charge field. The field is set zero at the points where no electrons are situated.

(d) $t=1.57 \mathrm{~ns}$ : The value of the radial field component is decreasing.

Generally we observe that the radial space charge field reaches values that are comparable to the longitudinal space charge field. The electron cloud is blown up transversely due to the repulsive radial space charge fields at the tip where the electron density is largest. At a later stage, when most electrons have left the gas gap or got attached, the radial space charge field has the opposite sign.

\subsubsection{Radial Drift Velocity}

The value of the $r$-component of the electric field $E_{r}(r, z, t)$ determines the radial spread of the electron cloud in an avalanche on top of the transverse diffusion. Fig. 

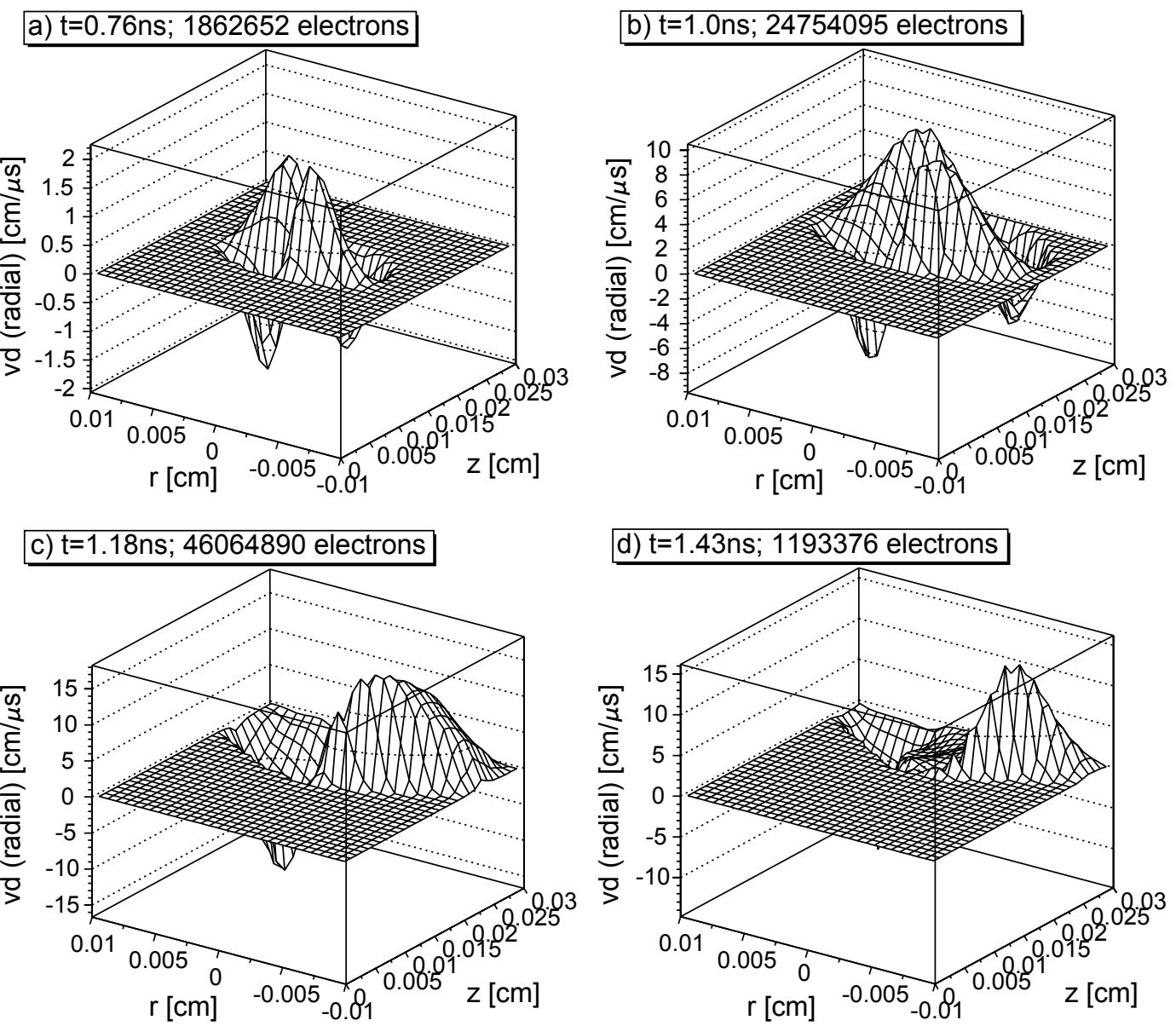

Figure 7.14: $r$-component of the radial component of the drift velocity $\left(v_{D}\right)_{r}(r, z, t)$ in an avalanche started by one electron at $(r=0, z=0)$ in a Timing RPC filled with the standard gas mixture and with an operating voltage of $2.8 \mathrm{kV}$.

7.14 shows the value of the radial component of the drift velocity $\left(v_{D}\right)_{r}(r, z, t)$ at different time steps and at the different grid points given by the $r$ - and $z$-coordinates. We simulated an avalanche that was started by a single electron at the position $r=0$, $z=0$ and at $t=0 \mathrm{~ns}$. We will frequently compare $\left(v_{D}\right)_{r}(r, z, t)$ to the driftvelocity of an electron at the applied electric field strength $v_{0}=v_{D}\left(E_{0} / p\right)$.

(a) At $t=0.76 \mathrm{~ns}$ the avalanche consists of around $1.9 \times 10^{6}$ electrons. The radial component of the drift velocity has values of up to $10 \%$ of the value of $v_{0}$. A positive value leads to the electrons being repelled towards larger values of $r$. As expected from the radial field distribution (see Fig. 7.12), the electrons drift away from the center of the avalanche at $r=0$ at the tip of the avalanche, while the electrons drift towards the center at the tail. 
(b) At $t=1.0 \mathrm{~ns}$ the maximum radial drift velocity reaches about $50 \%$ of the value of $v_{0}$.

(c) At $t=1.18 \mathrm{~ns}$ the avalanche has grown to around $4.6 \times 10^{7}$ electrons. Many electrons have already left the gas gap. The maximum radial drift velocity is about $75 \%$ of the value of $v_{0}$. The electrons drift towards the center of the avalanche at $r=0$ almost everywhere.

(d) At $t=1.43 \mathrm{~ns}$ the maximum radial drift velocity is still around $75 \%$ of the value of $v_{0}$. The remaining electrons in the regions with larger values of $r$ are drifting towards the center of the avalanche at $r=0$.

Since at the tip of the avalanche the electron density can be very large, the radial spread of the avalanche due to the space charge fields on top of the diffusion can become very large here.

\subsection{Avalanches in Pure Isobutane}

In this section we present results on the detailed simulation of avalanches in pure isobutane. Since isobutane is not electronegative, the attachment coefficient is zero for all values of the electric field strength and the effective Townsend coefficient equals the Townsend coefficient. Assuming a large gas gain, we expect that the Townsend coefficient is approximately zero at all positions in the gas gap where the space charge field decreases the total electric field strength such that it approaches zero. Since in Timing RPCs filled with the standard gas mixture $\mathrm{C}_{2} \mathrm{~F}_{4} \mathrm{H}_{2} / \mathrm{i}-\mathrm{C}_{4} \mathrm{H}_{10} / \mathrm{SF}_{6}(85 \%, 5 \%, 10 \%)$ we have seen that a large fraction of the electrons in the final stage of the avalanches get attached forming a negative ion, we expect a very different behaviour of the avalanches in pure isobutane.

We investigate the same detector geometry as in section 7.2 , the only difference being the gas mixture. The applied voltage is $2.6 \mathrm{kV}$, leading to an electric field of around $E_{0}=8.67 \mathrm{kV} / \mathrm{mm}$ in the gas gap. The pressure is $1013 \mathrm{mbar}$ and the temperature $296.15 \mathrm{~K}$. We simulate an avalanche that is started by a single electron at the position $r=0, z=0$ and at $t=0 \mathrm{~ns}$. We will show plots of the different parameters at different time steps and at the different grid points given by the $r$ - and $z$-coordinates. The charge induced by this avalanche is $40 \mathrm{fC}$ and the total signal charge is around $1 \mathrm{pC}$.

\subsubsection{Early Stage of the Avalanches}

Fig. 7.15 shows the values of different parameters at time $t=1.94 \mathrm{~ns}$ and at the different grid points given by the $r$ - and $z$-coordinates. The avalanche consists of 

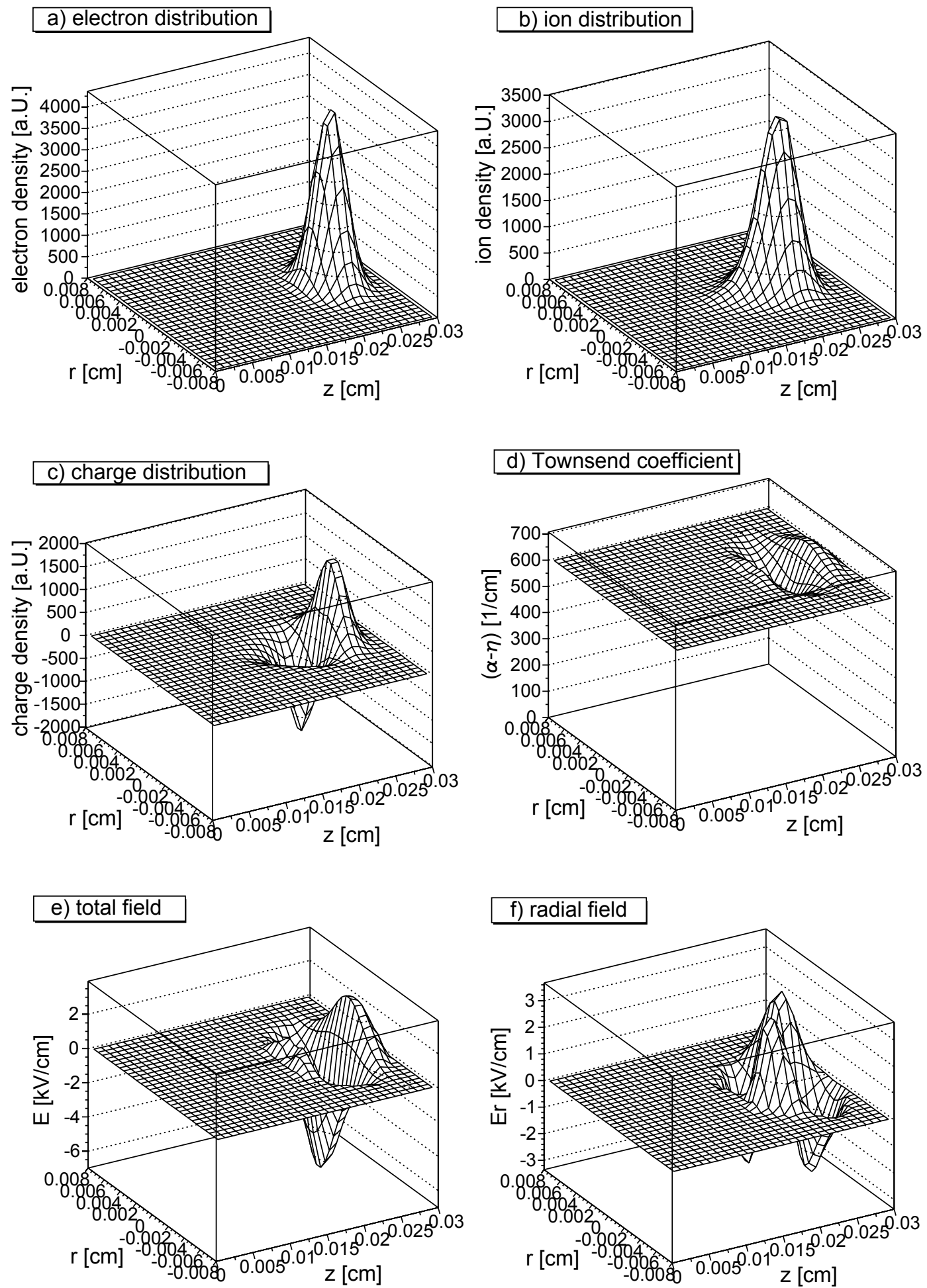

Figure 7.15: Several parameters in an avalanche in pure isobutane. $t=1.94 \mathrm{~ns}, 5.2 \times$ $10^{5}$ electrons. 
around $5.2 \times 10^{5}$ electrons and the first electrons just reach the anode. The drift velocity is around $v_{0}=v_{D}\left(E_{0}, p\right)=0.12 \mathrm{~mm} / \mathrm{ns}$, which is only $60 \%$ of the drift velocity of the avalanches in section 7.2 .

(a) The electron distribution is roughly Gaussian.

(b) The ion distribution represents an approximate exponential avalanche growth combined with Gaussian diffusion.

(c) The plot shows the density of negative charge: At the tip of the avalanche it is positive due to the majority of electrons here. At the tail it is negative due to the amount of positively charged ions that stay behind .

(d) At this stage the Townsend coefficient is altered by up to $10 \%$ at some positions in the gas gap.

(e) The total electric field strength sensed by the electrons and contributed by the avalanche space charge is about $3 \%$ of the applied electric field strength $E_{0}$.

(f) The same is true for the radial electric field strength sensed by the electrons. As before, the radial electric field repels electrons from the center of the avalanche at the tip of the charge distribution, while it attracts electrons towards the center at the tail.

The space charge effect is not very strong at this stage of the avalanche.

\subsubsection{Later Stages of the Avalanches}

Fig. 7.16 shows the values of the parameters at time $t=2.37 \mathrm{~ns}$. The avalanche consists of around $3.1 \times 10^{6}$ electrons at that stage.

(a) Many electrons have already entered the anode. The anode is conductive and the electrons disappear instantly as soon as they leave the gas gap.

(b) The ion density is largest in the center of the avalanche in front of the anode.

(c) The density of the negative charge: Since many electrons have already left the gas gap, this value is negative at most positions.

(d) The Townsend coefficient is increased by around 50\% at the maximum and decreased to around a third of the value at $E_{0}$. The maximum is at the tail of the avalanche. 

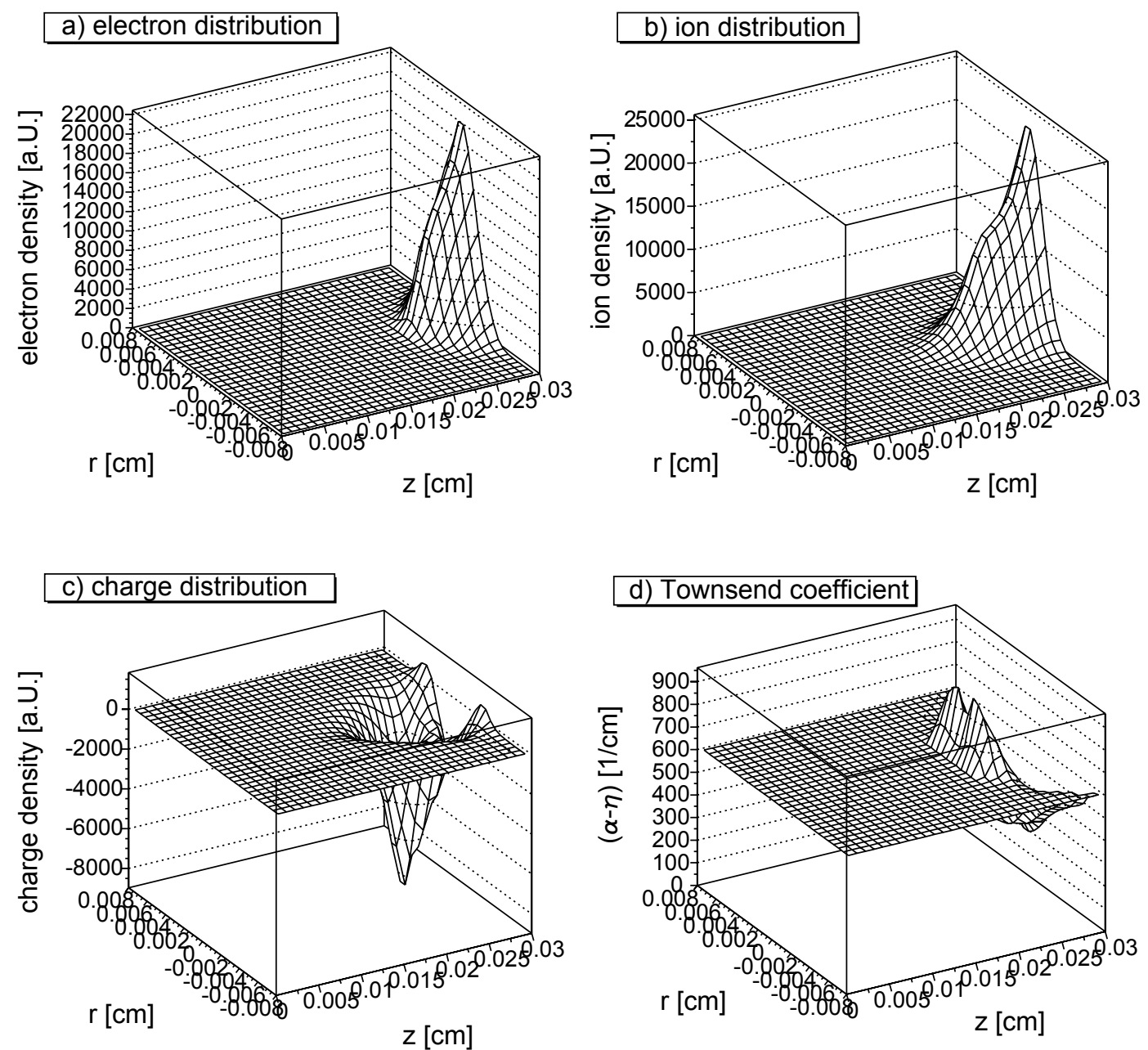

\section{e) total field}
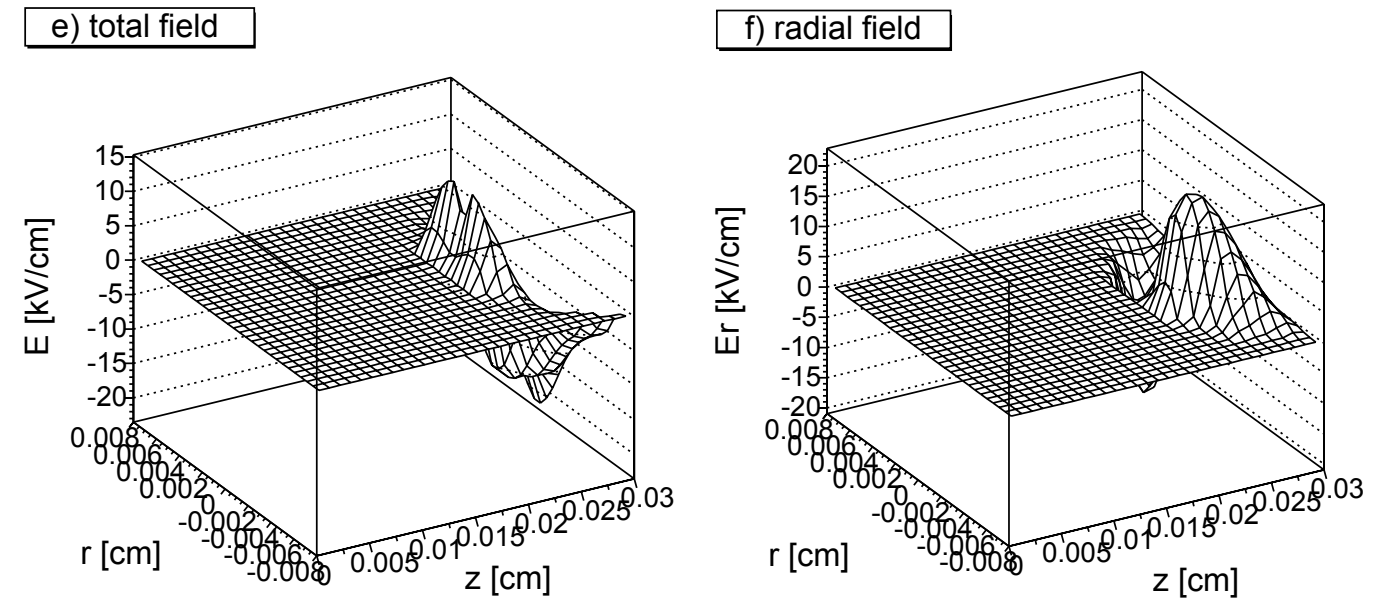

Figure 7.16: Several parameters in an avalanche in pure isobutane. $t=2.37 \mathrm{~ns}, 3.1 \times$ $10^{6}$ electrons. 
(e) The total electric field sensed by the electrons is increased by around $15 \%$ as compared to $E_{0}$ at the maximum. At its minimum it is decreased by around $25 \%$.

(f) The radial electric field strength sensed by the electrons reaches values up to $25 \%$ of $E_{0}$. At this stage of the avalanche it attracts electrons towards the center of the avalanche at most positions in the gas gap.

Fig. 7.17 shows the values of the parameters at time $t=3.13 \mathrm{~ns}$. The avalanche size has decreased to around $4.4 \times 10^{5}$ electrons.

(a) We observe that at the 'outer' positions at larger values of $r$ the electrons have disappeared already while in the center of the avalanche at $r \approx 0$ the electron density is still almost as large as in Fig. 7.16.

(b) The shape of the ion distribution is altered somewhat, because at some positions the amount of ions increases due to a Townsend coefficient that can still have non-zero values at some positions. This happens mainly in the regions a little further from the cathode.

(c) The density of negative charges in the gas gap has negative values everywhere in the gas gap, since the positive ions outnumber the electrons everywhere.

(d) The Townsend coefficient has a value of approximately zero almost everywhere, where we find electrons.

(e) At the minimum the value of the total electric field contributed by the avalanche space charge has the same value as $E_{0}$. These are the positions where the Townsend coefficient approaches zero. The same is true for the drift velocity which is not plotted.

(f) The radial electric field strength sensed by the electrons reaches values up to $35 \%$ of $E_{0}$ and attracts the electrons towards the center of the avalanche at $r=0$.

\subsection{Summary}

We carried out detailed simulations of avalanches started by a single electron in Timing RPCs with $0.3 \mathrm{~mm}$ gas gaps. We find that the radial electric field contributed by the avalanche charges reaches the same order of magnitude as the longitudinal space charge field and as the applied electric field. As a consequence, the radial spread of the avalanche due to the repulsion and attraction of the charges is very large. As the electron clusters drift through the gap, they are blowed up transversely at their tip and 

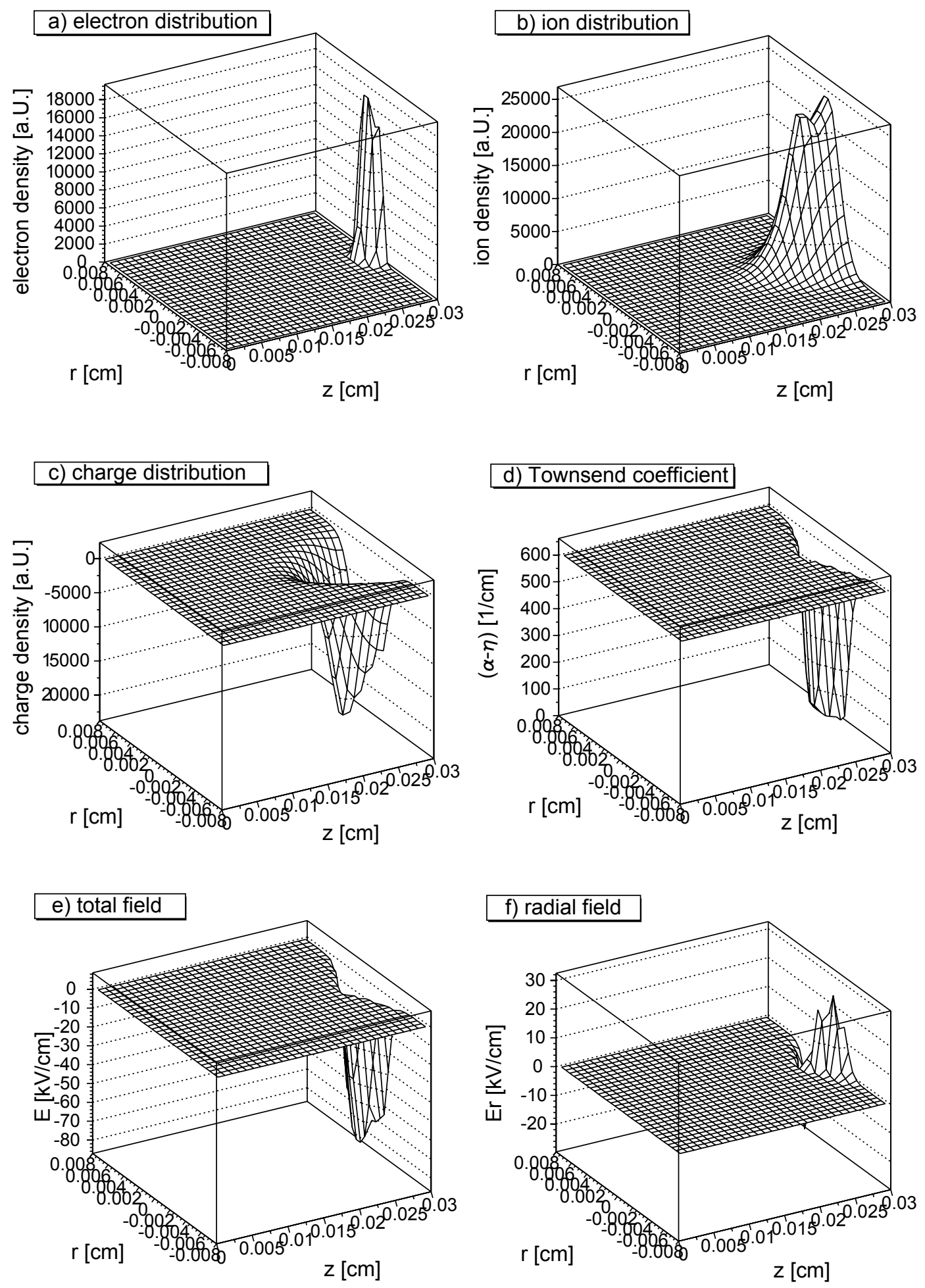

Figure 7.17: Several parameters in an avalanche in pure isobutane. $t=3.13 \mathrm{~ns}, 4.4 \times$ $10^{5}$ electrons. 
contracted at their tail by this effect. At a later stage, where the cluster has reached the anode, the contraction preponderates. In the standard gas the largest part of the electrons get attached at the final stage in the central region. In isobutane regions are formed at that stage, where the propagation and multiplication of the electron clouds is coming to a halt. The electrons can then only be removed if the electric field slowly changes due to the ions drifting away. 


\section{Chapter 8}

\section{Conclusions and Outlook}

Comprehensive summaries of the introduction, the description of the detector physics of RPCs, of the Monte-Carlo avalanche simulation programs and of the results that were obtained with the simulations are given at the end of the corresponding chapters, in sections [1.6, 2.6, 3.5, 5.3, 6.7 and 7.4.

We have applied standard detector physics simulations to RPCs and find good agreement with measurements. While from the simple electric field configuration found in the gas gap of an RPC one might expect a just as simple description of the avalanche propagation, it turns out that the physical processes are very complex. The detailed simulation of the signal development demands for the dynamic calculation of the electric field that is sensed by the electrons in the avalanche and that is contributed by the positive and negative avalanche charges. Especially at the final stages of the avalanche development this space charge field can easily reach the same strength as the applied electric field. We can roughly divide this field into three zones: At the two zones at the tip and at the tail of the electron distribution the total electric field is increased by the space charge field. At the center of the avalanche, where most of the electrons are situated, the total electric field is strongly decreased. Assuming an avalanche in an electronegative gas, we find negative values for the effective Townsend coefficient and thus strong attachment here. At a later stage, when the electron cloud has reached the anode, almost all electrons are situated in that zone of extremely lowered field strength and attachment effects dominate. Moreover, we find that the radial electric field contributions of the space charge are within the same order of magnitude as the applied electric field. Thus the influence of this effect on the radial spread of the avalanches at large gas gain is large.

The main result is that the experimentally observed efficiencies, time resolution and the average avalanche charges can be explained with standard detector physics and the values of the gas parameters as predicted by HEED, MAGBOLTZ and IMONTE. The detector behaviour is well understood. We find that the efficiencies of single gap Timing RPCs with $0.3 \mathrm{~mm}$ gas gap (around $75 \%$ ) are explained by a large primary 
ionization density (about 9.5 cluster/cm) together with a very large effective Townsend coefficient of about $113 / \mathrm{mm}$. Secondary particles produced in the detector frame should not play an important role in the RPC behaviour. The space charge effect indeed suppresses the large avalanche charges predicted by the avalanche statistics, which in the simulations also leads to shapes of the charge spectra very similar to measurements. Contrary to wire chambers, RPCs operate in a strong space charge mode. The experimental result of a first exponential and then linear dependence of the average charges on the high voltage is reproduced by the simulations.

Since the rate capability of RPCs is closely connected to the resistivity of the electrode material, as a next step a careful study of the influence of the charges inside the resistive electrodes on the electric field inside the gas gap could lead to a better understanding of these limitations and might open ways to optimize the detector for high rate applications. The electrostatic potential solutions that would be needed for these investigations are available. 


\section{Appendix A}

\section{Differential Collision Cross Sections}

In this appendix we list theoretical expressions for the differential collision cross sections for charged particles with free electrons, which give the probability that the particle with energy $E$ lose an energy between $E^{\prime}$ and $E^{\prime}+d E^{\prime}$ in the collision. Magnetic moment and spin interactions are included. The constant $C$ is given by $C=2 \pi Z z r_{e} m_{e} c^{2}$, where $Z$ is the atomic number of the material, $z$ is the charge of the incident particle in unit charges, $m_{e}$ is the electron mass, $c$ is the speed of light, $r_{e}=e^{2} / 4 \pi \epsilon_{0} m_{e} c^{2}$ is the classical electron radius and $\epsilon_{0}$ the dielectric constant of vacuum.

\section{A.1 Electron-Electron Scattering}

The collision cross section of electrons with electrons

$$
e^{-}+e^{-} \rightarrow\left(e^{-}\right)^{\prime}+\left(e^{-}\right)^{\prime}
$$

has been calculated by Möller [108] on the basis of the Dirac Theory. When the energy $E$ of the primary particle is large compared with $m_{e} c^{2}$ (therefore $\beta \approx 1$ ), the collision cross section is

$$
\left.\frac{d \sigma}{d E^{\prime}}\right|_{c o l}=\frac{C E^{2}}{\left(E-E^{\prime}\right)^{2}\left(E^{\prime}\right)^{2}}\left[1-\frac{E^{\prime}}{E}+\left(\frac{E^{\prime}}{E}\right)^{2}\right]^{2}
$$

Since one can not distinguish between primary and secondary particle after the collision, Eq. A.1 is interpreted as giving the probability of a collision that leaves one electron in the energy state $E^{\prime}$ and the other in $E-E^{\prime}$. All possible cases are taken into account by letting the energy $E^{\prime}$ vary from 0 to $E / 2$ [15]. 


\section{A.2 Positron-Electron Scattering}

Bhabha [109] has calculated the collision cross section of positrons with electrons

$$
e^{+}+e^{-} \rightarrow\left(e^{+}\right)^{\prime}+\left(e^{-}\right)^{\prime} .
$$

For $E \gg m_{e} c^{2}$ the probability of a collision after which the electron has an energy in $E^{\prime}$ and $E^{\prime}+d E^{\prime}$ is

$$
\left.\frac{d \sigma}{d E^{\prime}}\right|_{c o l}=\frac{C}{\left(E^{\prime}\right)^{2}}\left[1-\frac{E^{\prime}}{E}+\left(\frac{E^{\prime}}{E}\right)^{2}\right]^{2} .
$$

Accordingly the cross section of a collision after which the positron has an energy in $E^{\prime}$ and $E^{\prime}+d E^{\prime}$ is

$$
\left.\frac{d \sigma}{d E^{\prime}}\right|_{c o l}=\frac{C}{\left(E-E^{\prime}\right)^{2}}\left[1-\frac{E^{\prime}}{E}+\left(\frac{E^{\prime}}{E}\right)^{2}\right]^{2} .
$$

The total cross section for a positron-electron collision after which either the positron or the electron has an energy in $E^{\prime}$ and $E^{\prime}+d E^{\prime}$ can be calculated by multiplying Eq. A.2 with

$$
\frac{E^{2}}{\left(E-E^{\prime}\right)^{2}}\left[1-2 \frac{E^{\prime}}{E}+2\left(\frac{E^{\prime}}{E}\right)^{2}\right] .
$$

Again one takes into account all possible cases by letting the energy $E^{\prime}$ vary from 0 to $E / 2$ [15].

\section{A.3 Scattering of Massive Spin 0 Particles off Electrons}

Bhabha [110] has calculated the collision cross section for particles with mass $m$ and spin 0 , e.g.

$$
K^{-}+e^{-} \rightarrow\left(K^{-}\right)^{\prime}+\left(e^{-}\right)^{\prime}
$$

as

$$
\left.\frac{d \sigma}{d E^{\prime}}\right|_{c o l}=\frac{C}{\beta^{2}\left(E^{\prime}\right)^{2}}\left[1-\beta^{2} \frac{E^{\prime}}{E_{\max }}\right]
$$

where $E_{\text {max }}^{\prime}$ is the maximum transferable energy from Eq. 1.6. 


\section{A.4 Scattering of Massive Spin $\frac{1}{2}$ Particles off Electrons}

Bhabha [110] and Massey and Corben [111] have calculated the collision cross section for particles with mass $m$ and spin $\frac{1}{2}$, e.g.

$$
\mu^{+}+e^{-} \rightarrow\left(\mu^{+}\right)^{\prime}+\left(e^{-}\right)^{\prime}
$$

as

$$
\left.\frac{d \sigma}{d E^{\prime}}\right|_{c o l}=\frac{C}{\beta^{2}\left(E^{\prime}\right)^{2}}\left[1-\beta^{2} \frac{E^{\prime}}{E_{\max }}+\frac{1}{2}\left(\frac{E^{\prime}}{E+m c^{2}}\right)^{2}\right] .
$$

\section{A.5 Scattering of Massive Spin 1 Particles off Electrons}

The collision cross section for particles with mass $\mathrm{m}$ and spin 1 was calculated by Massey and Corben [111] and by Oppenheimer et al. [112]. It can be obtained by replacing the square brackets in Eq. A.6 with

$$
\left[\left(1-\beta^{2} \frac{E^{\prime}}{E_{\max }}\right)\left(1+\frac{1}{3} \frac{E^{\prime}}{E_{c}}\right)+\frac{1}{3}\left(\frac{E^{\prime}}{E+m c^{2}}\right)^{2}\left(1+\frac{1}{2} \frac{E^{\prime}}{E_{c}}\right)\right],
$$

where

$$
E_{c}=\frac{m^{2} c^{2}}{m_{e}}
$$

\section{A.6 Collision Cross Section Dependence on the Spin}

For a distant collision, when $E^{\prime} \ll E_{\max }, E^{\prime} \ll E$ and $E^{\prime} \ll E_{c}$, the collision cross sections given by Eqs. A.1, A.4, A.5, A.6 and A.7 all reduce to

$$
\left.\frac{d \sigma}{d E^{\prime}}\right|_{c o l}=\frac{C}{\beta^{2}} \frac{1}{\left(E^{\prime}\right)^{2}}
$$

Thus, at the limit of small values of the transfered energy $E^{\prime}$, the collision probabilities of different kinds of particles become identical and depend only on the energy $E^{\prime}$ 
of the secondary electron and on the velocity $\beta$ of the incident particle. Eq. A.9 can be derived based on relativistic mechanics [15] and represents the Coulomb interaction.

The influence of the spin manifests itself only for very close collisions, when $E^{\prime}$ is comparable to $E$ and $E_{c}$. At high values of $E^{\prime}$ the collision cross section is an increasing function of the spin. This can be seen if we consider, in particular, the case $E^{\prime} \ll E_{\text {max }}, E^{\prime} \ll E$. Then the collision cross section for spin 0 and spin $\frac{1}{2}$ particles is given by Eq. A.9. If $E^{\prime}$ is comparable to $E_{c}$, the collision cross section for spin 1 particles becomes

$$
\left.\frac{d \sigma}{d E^{\prime}}\right|_{c o l}=\frac{C}{\beta^{2}} \frac{1}{\left(E^{\prime}\right)^{2}}\left(1+\frac{1}{3} \frac{E^{\prime}}{E_{c}}\right)
$$

The expression contains an additional term so that it decreases with increasing energy as $1 / E^{\prime}$, whereas Eq. A.9 decreases as $1 /\left(E^{\prime}\right)^{2}$. For $E^{\prime}>3 E_{c}$ the interaction due to the spin exceeds the Coulomb interaction. 


\section{Appendix B}

\section{Überblick}

Widerstandsplattenkammern (im englischen Resistive Plate Chambers oder abgekürzt $R P C s$ ) sind Teilchendetektoren, die in heutigen und zukünftigen Hochenergiephysikexperimenten auf großen Flächen eingesetzt werden. Sie bestehen aus zwei parallelen Elektrodenplatten, die einen gasgefüllten Spalt von wenigen hundert Micrometern bis einigen Millimetern umschließen. Wenigstens eine der beiden Elektroden besteht aus einem Material mit hohem Volumenwiderstand von $10^{7}$ bis $10^{12} \Omega \mathrm{cm}$. RPCs erreichen eine gute Zeitauflösung (bis zu 50 ps) und eine hohe Nachweiseffizienz ( $\approx 99 \%$ für mehrere kombinierte Zähler). Außerdem sind sie technisch sehr einfach aufgebaut.

RPCs wurden zu Beginn der achtziger Jahre von R. Santonico und R. Cardarelli entwickelt [36, 37]. Ihre Funktionsweise beruht auf dem Energieverlust des primären geladenen Teilchens durch Kollisionen mit Gasatomen im Gasspalt des Detektors, wodurch einige der Atome ionisiert werden. Durch eine an die Elektroden angelegte Hochspannung entsteht ein starkes homogenes elektrisches Feld im Gasspalt, in welchem die freien Elektronen zur Anode hin beschleunigt werden. Durch Kollisionen mit weiteren Atomen vermehren sich die Elektronen, und die Bewegung dieser Elektronenlawinen induziert einen Strom auf einer externen Ausleseelektrode. Bei hoher Gasverstärkung von etwa $10^{8}$ ändert sich die Dynamik der Lawinenpropagation. Dann tragen verstärkt Photonen zur Ausbreitung der Lawinen bei: Es entstehen Streamer. Wenn diese Streamer die beiden Elektroden erreichen, kann es passieren, dass ein leitender Kanal entsteht, durch den sich die Elektroden entladen können (Kanalaufbau [38]). An dieser Stelle erklärt sich der Sinn der Resistivität des Elektrodenmaterials: Die Entladung beschränkt sich auf eine kleine Fläche rund um die ursprüngliche Ladungslawine, und ihre Energie bleibt begrenzt; der Streamer löscht sich selbst. Auf dieser Fläche ist der Detektor nicht ansprechbereit, bis die Elektroden lokal wieder aufgeladen sind. Die Zeitkonstante für diesen Prozess kann je nach dem Wert des Volumenwiderstands bis zu eine Sekunde betragen. Durch die Resistivität der Elektroden wird zum einen verhindert, dass ein energiereicher Funken die lokale Elektrodenoberfläche in Mitleidenschaft zieht. Zum anderen bleibt der Detektor auf der restlichen Fläche ansprechbereit. Allerdings führt sie auch zu einer eingeschränkten 
Ratentauglichkeit.

RPCs wurden ursprünglich im Streamermodus betrieben, was zu großen Signalhöhen führt, und die Anforderungen an die Ausleseelektronik und die Genauigkeit des Elektrodenabstandes vereinfacht. Um verbesserte Hochratenfestigkeit und verminderte Alterung der RPCs zu erlangen, wurde der Betrieb im Lawinenmodus populär. Diese Entwicklung wurde möglich durch die Einführung neuer Gasmischungen auf der Basis von $\mathrm{C}_{2} \mathrm{~F}_{4} \mathrm{H}_{2}$ mit geringen $\mathrm{SF}_{6}$-Beimischungen [39]. Während Streamer schwer zu studieren sind, eröffnete der Lawinenmodus die Möglichkeit detaillierter Simulationen der physikalischen Prozesse in RPCs. Als Beispiele für die Verwendung vom Lawinenmodus-RPCs können die beiden am Beschleuniger LHC] am Europäischen Kernforschungszentrum CERN in Genf sich im Aufbau befindenden Experimente AT-

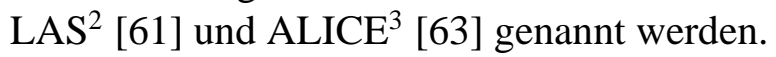

Im Muonensystem von ATLAS werden RPCs mit $2 \mathrm{~mm}$ Plattenabstand betrieben im Lawinenmodus auf einer Fläche von $3650 \mathrm{~m}^{2}$ und mit 355.000 unabhängigen Auslesekanälen verwendet [54]. Muonen mit großen Transversalimpulsen sind unter den vielversprechendsten Signaturen für die Physik der Proton-Proton Kollisionen am LHC. So könnten vier simultan auftretende Muonen auf den Zerfall eines der gesuchten Higgs-Teilchen $H$ hindeuten: $H \rightarrow Z+Z \rightarrow \mu^{+}+\mu^{-}+\mu^{+}+\mu^{-}$. Anforderungen an den verwendeten Detektor sind unter anderem eine Zeitauflösung von etwa $1 \mathrm{~ns}$, was von RPCs leicht erreicht wird. Die verwendete Geometrie wird Trigger RPC genannt. Betrieben im Lawinenmodus erreichen diese Detektoren eine Nachweiseffizienz von 98.5\% für einen Spalt bis zu einer Teilchenrate von einigen $\mathrm{kHz} / \mathrm{cm}^{2}$.

In ALICE werden RPCs mit $0.25 \mathrm{~mm}$ Plattenabstand in Mehrfach-Spalt Konfigurationen [56] auf einer Fläche von $176 \mathrm{~m}^{2}$ mit 160.000 individuellen Auslesezellen zur Flugzeitmessung implementiert [33]. Bei den extremen Teilchenmultiplizitäten, die für zentrale Blei-Blei Stöße am LHC vorhergesagt werden, ist die Teilchenidentifizierung eine wichtige Aufgabe. Um eine Separation von Kaonen und Pionen mit drei Standardabweichungen Genauigkeit zu erreichen, sollte die Zeitauflösung des Detektors 90 ps erreichen, was von den genannten RPCs erreicht wird. Die Leistungsmerkmale dieser Timing RPCs genannten Detektoren sind vergleichbar mit herkömmlichen Detektoren auf der Basis von Szintillatoren, sie bieten jedoch einen Preis pro Kanal, der bis zu einer Größenordnung niedriger ist.

${ }^{1}$ Large Hadron Collider

${ }^{2}$ A Toroidal LHC ApparatuS

${ }^{3}$ A Large Ion Collider Experiment 


\section{B.1 Motivation für die Arbeit}

Trotz des umfangreichen Einsatzes dieser Detektortechnologie sind einige experimentelle Ergebnisse noch nicht genau verstanden worden. Insbesondere hinsichtlich der Erklärung der guten Nachweiseffizienz von Timing RPCs kamen vielerlei Fragen auf. Unter anderem wurde vorgeschlagen, die hohe Effizienz anhand von Begleitelektronen zu erklären, die vom Primärteilchen aus dem Detektorrahmen gelöst werden. Verneint man dies, so muss man von einer hohen Dichte von primären Ionisationszentren, aber auch von einer sehr großen Gasvertärkung ausgehen. Die hohe Ionisationsdichte führt dazu, dass sich Primärelektronen mit einer hohen Warscheinlichkeit nahe an der Kathode befinden. Von hier durchqueren die Ladungslawinen den gesamten Spalt und aufgrund des exponentiellen Wachstums erreichen sie eine ausreichende Größe, um nachgewiesen zu werden. Auch im Falle der hohen Ionisationsdichte muss die Gasverstärkung sehr groß sein, damit möglichst viele Lawinen den gesetzten Schwellwert erreichen. In diesem Falle ist aber ein sehr starker Raumladungseffekt nötig, um die gemessenen kleinen Ladungen um $1 \mathrm{pC}$ und den dazu nötigen Unterdrückungsfaktor teilweise von bis $\mathrm{zu} 10^{7} \mathrm{zu}$ erklären. Der Begriff Raumladungseffekt beschreibt den Prozess der dynamischen Verzerrung des angelegten elektrischen Feldes durch die Ladungsträger in der Lawine. Dieses zusätzliche Feld hat einen starken Einfluss auf die Driftgeschwindigkeit und Multiplikation der Elektronen. Um die großen Werte für die Lawinenladungen wirkungsvoll zu unterdrücken, muss der Raumladungseffekt eine gewisse Stärke haben und das elektrische Feld an den Positionen, an denen sich der Großteil der Elektronen in der Lawine befindet, stark erniedrigen. Dann muss aber das Feld an anderen Positionen durch den gleichen Effekt stark erhöht sein. Viele Autoren lehnen die Möglichkeit ab, dass sich eine Lawine unter diesen extremen Umständen ausbreiten kann, ohne sich an den Stellen erhöhter Feldstärke in einen Streamer umzuwandeln.

Weitere Fragen betreffen die Form der experimentell gemessenen Ladungsspektren. Man beobachtet einen Scheitelpunkt in den Spektren von Trigger RPCs, der zu höheren Spannungen hin ausgeprägter wird. Die Statistik der Primärionisation und der Ladungsmultiplikation sollte jedoch zu einer Form der Spektren führen die monoton zu höheren Ladungen hin abfällt.

\section{B.2 Detektorphysik von RPCs}

Die zur kompletten Beschreibung der Erzeugung und Evolution von Ladungslawinen und Signalen in RPCs benötigten Parameter sind

- die mittlere freie Weglänge zwischen zwei ionisierenden Kollisionen $\lambda$,

- die Zufallsverteilung für die Anzahl der Elektronen pro Cluster, 
- der Townsend-Koeffizient $\alpha(E / p)$,

- der Elektronenanlagerungskoeffizient $\eta(E / p)$,

- die Elektron-Driftgeschwindigkeit $v_{D}(E / p)$,

- der transversale and der longitudinale Diffusionskoeffizienten $D_{T}(E / p)$ und $D_{L}(E / p)$,

- das Potenzial einer Punktladung im Gasspalt einer RPC und

- der Wert der $z$-Komponente $\left.\right|^{\natural}$ des Wichtungsfeldes $\vec{E}_{W}(\vec{r})$ in der zentralen Schicht der genannten Geometrie.

Die Werte von $\lambda$ und die Clustergrößenverteilung können mit dem Simulationsprogramm HEED [25] für ionisierende Teilchen verschiedener Art und Energie berechnet werden. Die Werte von $\alpha(E / p), \eta(E / p), v_{D}(E / p), D_{T}(E / p)$ und $D_{L}(E / p)$ sind Funktionen des elektrischen Feldes $E$ und des Gasdruckes $p$ und können mit den Programmen MAGBOLTZ [76] und IMONTE [77] berechnet werden.

Die Distanz zwischen zwei ionisierenden Kollisionen des Primärteilchens mit den Gasatomen ist exponentialverteilt um $\lambda$. Dann ist die Anzahl der ionisierenden Ereignisse in einem Spalt der Dicke $g$ Poissonverteilt um $\bar{n}=g / \lambda$. Die maximale Effizienz einer RPC ist durch $\epsilon_{\max }=1-\exp (-\bar{n})$ gegeben. Hier ist $\exp (-\bar{n})$ die Warscheinlichkeit dafür , kein Cluster im Gasspalt zu finden. $\epsilon_{\max }$ hängt stark vom verwendeten Gas und von $g$ ab. Die Clustergrößenverteilung hat einen Mittelwert von einigen Elektronen, jedoch ergibt sich auch eine gewisse Warscheinlichkeit, bis zu einige hundert Elektronen in einem Cluster zu finden.

In einem homogenen elektrischen Feld kann die Propagation einer Elektronenwolke durch die Diffusionsbewegung und eine überlagerte konstante Driftbewegung beschrieben werden. Für die Fluktuationen in der Ladungsmultiplikation wird ein Modell von W. Legler [85] verwendet, welches die Statistik der Elektronenlawinen in elektronegativen Gasen bei hohen Feldstärken und bei hoher Gasverstärkung beschreibt. Die Verteilung hängt explizit von den Werten von $\alpha(E / p)$ und $\eta(E / p)$ ab. Weiterhin wird für die Berechnung des Raumladungsfeldes eine analytische Lösung für das Potenzial einer Punktladung in einem unendlich ausgedehnten Plattenkondensator mit drei homogenen dielektrischen Schichten verwendet [3, 4]. Dieses Potenzial kann gut durch die Potenziale einer freien Punktladung sowie einer Spiegelladung, welche sich in der näheren Elektrode befindet, approximiert werden. Schließlich wird der induzierte Strom $i(t)$ von $N(t)$ Einheitsladungen $e_{0}$, die sich mit der Geschwindigkeit $\vec{v}_{D}(t)$ zur Zeit $t$ bewegen, mit Hilfe des Wichtungsfeld-Formalismus berechnet:

\footnotetext{
${ }^{4}$ Die $z$-Achse liegt senkrecht zu dem Detektorplatten. Die Elektronenlawinen breiten sich also parallel zur $z$-Achse in Richtung der Anode aus.
} 
$i(t)=\vec{E}_{w} \cdot \vec{v}_{D}(t) e_{0} N(t)$. Eine Ausleseelektronik kann simuliert werden, indem das Stromsignal mit der Impulsantwort des Systems gefaltet wird.

Basierend auf diesen physikalischen Prozessen werden vier Monte-Carlo Simulationsroutinen vorgestellt, wobei das $1-D$-Modell in [1] diskutiert wird und die $1.5-D$-, 2-D- und 3-D-Modelle Gegenstand dieser Doktorarbeit sind.

Das 1-D-Modell simuliert die longitudinale Ausbreitung der Lawinen entlang der $z$-Achse, welche in etliche Intervalle unterteilt wird. Die Primärcluster werden auf diese Intervalle verteilt und die Elektronen sodann in Richtung der Anode propagiert und anhand der beschriebenen Verteilung multipliziert, wobei die Werte von $\alpha(E / p)$ und $\eta(E / p)$ konstant angenommen werden. Eine Saturation aufgrund eines Raumladungseffektes kann simuliert werden, indem das Wachstum der Lawinen gestoppt wird, sobald diese eine bestimmten Größe erreichen. Dieses Modell wird zur detaillierten Untersuchung von Zeitauflösungen und Effizienzen von RPCs verwendet.

Im 1.5-D-Modell wird der Raumladungseffekt mit eingebunden, indem angenommen wird, dass die Lawinenladungen in transversaler Richtung in Scheiben untergebracht sind, die eine Ladungsverteilung tragen, welche Gaußförmig ist. Die Standardabweichung dieser Verteilungen hängt dabei fest vom transversalen Diffusionskoeffizienten und von der Distanz ab, die die Elektronen gedriftet sind. Wir verwenden die erwähnte analytische Lösung für das Potenzial einer Punktladung in der RPC. Das Integral über diese Lösung und die radiale Ladungsverteilung ergibt das elektrische Feld einer Scheibe mit dieser Ladungsverteilung. Die Summe über alle Scheiben ergibt das elektrische Feld der gesamten Raumladung der Lawine. Die Diffusionskoeffizienten $D_{T}$ und $D_{L}$ werden als konstant angenommen. Der Name "1.5-D-Modell" rührt daher, dass die Lawinenpropagation zwar ebenso wie beim 1-D-Modell nur in einer Dimension, nähmlich longitudinal, simuliert wird, die transversale Diffusion jedoch über die Berechnung des Raumladungsfeldes auch mit berücksichtigt wird. Das beschriebene Modell erlaubt die Berechnung von Ladungsspektren sowie des Einlusses des Raumladungseffektes auf die Signalform.

Das 2-D-Modell erlaubt auch die Simulation des radialen Raumladungseffektes. Unter der Annahme, dass die Lawinen einer Zylindersymmetrie unterliegen, wird der Gasspalt in ein Netz der longitudinalen und radialen Koordinaten $z$ und $r$ geteilt. Die Raumladung ist dann in Ringen der Größe $\delta r$ und $\delta z$ zentriert um die $z$-Achse untergebracht. Das Modell erlaubt die sehr detaillierte Simulation einzelner Lawinen.

Auch ein dreidimensionales Modell (3-D-Modell) wird vorgestellt. Es zeigt sich jedoch, dass die Intervalle sehr klein gewählt werden müssen, und damit die Rechendauer in nicht realisierbare Größen steigt, so dass dieses Modell nicht praktikabel ist. Allerdings bestätigen die Untersuchungen die Annahme einer Zylindersymmetrie, so dass die Verwendung des 2-D-Modells durchaus gerechtfertigt ist. 


\section{B.3 Ergebnisse}

Die Monte-Carlo Simulationen der Effizienzen und Zeitauflösungen von Trigger und Timing RPCs führen zu Resultaten, die sehr Nahe an gemessenen Werten liegen. Tatsächlich wird die gute Effizienz der Timing RPC durch eine hohe Dichte von Primärionisationszentren (etwa $9.5 / \mathrm{cm}$ ) und durch einen hohen effektive Townsend-Koeffizienten (etwa $113 / \mathrm{mm}$ ) erklärt. Werden Raumladungseffekte nicht berücksichtigt, so erhält man wie eingangs besprochen Mittelwerte für die Ladungen, die teilweise sieben Größenordnungen über den experimentell beobachteten liegen. Mit dem 1.5D-Modell unter Berücksichtigung des Raumladungseffektes berechnete Ladungsspektren haben Mittelwerte, die im Vergleich dazu nahe an den Messungen liegen. Die Form der Spektren entspricht sehr genau den Messungen. Die RPC wird im Gegensatz zu Drahtkammern in einem Raumladungsmodus betrieben, welcher sich über einen großen Bereich der angelegten Spannung erstreckt, und die großen Werte der Ladungen wirkungsvoll unterdrückt. Das Wachstum der Lawinen ist nur zu Beginn exponentiell. $\mathrm{Zu}$ einem späteren Zeitpunkt weicht der Signalanstieg aufgrund des Raumladungseffektes von einem exponentiellen ab. Dies wirkt sich schon auf dem Schwellwert-Niveau aus. Die Zeitauflösung wird jedoch nicht vom Raumladungseffekt beeinträchtigt.

Die Korrelation der Lawinenladungen zur Zeit der Schwellwertüberschreitung (Ladung-Zeit-Korrelation) wird in Experimenten verwendet um die Zeitauflösung von Timing RPCs zu verbessern. Sie wird beeinflusst von der Verstärkerelektronik und von intrinsischen Detektoreffekten. Betrachtet man die intrinsische Korrelation, so findet man, dass besonders die Signale mit langsamer Anstiegszeit eine Korrelation zur Ladung zeigen.

Das longitudinale Raumladungsfeld erreicht die gleiche Größenordnung wie das extern angelegte Feld. Elektronenanlagerung spielt eine sehr große Rolle, besonders im Endstadium der Lawinen. Da keine photonischen Effekte in der Simulation enthalten sind, wird erwartungsgemäß das Auftreten von Streamern nicht reproduziert. Ein Teil der Ergebnisse, die mit dem 1.5-D-Modell erhalten wurden, wurde bereits publiziert [5, 6].

Berechnungen mit dem 2-D-Modell ergeben, dass auch das radiale Raumladungsfeld in der Größenordnung des angelegten Feldes liegen kann. Zunächst werden die Elektronenwolken radial an ihrem vorderen Ende (in Richtung der Anode) durch dieses Feld aufgebläht und an ihrem hinteren Ende zusammengezogen. $\mathrm{Zu}$ einem späteren Zeitpunkt, wenn die Elektronenwolke die Anode erreicht hat, zieht das radiale Raumladungsfeld die Elektronenwolke überall zum Zentrum hin zusammen. 


\section{B.4 Schlussfolgerung und Ausblick}

Die Anwendung von Standard Detektorphysik Prozessen auf die Simulation von Ladungslawinen in RPCs führt zu Ergebnissen, die gut mit experimentellen Resultaten übereinstimmen. Obwohl die RPC technisch sehr einfach aufgebaut ist, und obwohl die Feldgeometrie sehr einfach ist, zeigt sich, dass die Prozesse, die während der Ladungslawine ablaufen (Raumladungseffekte), sehr komplex sind.

Eine nähere Untersuchung des Einflusses der abfließenden Ladungen in den resistiven Elektroden auf das elektrische Feld im Gasspalt wäre ein nächster Schritt, um die Ratenfähigkeit des Detektors besser zu verstehen und zu optimieren. Die dazu benötigten elektrostatischen Lösungen sind vorhanden [3]. 


\section{Bibliography}

[1] W. Riegler, R. Veenhof, and C. Lippmann. Simulation of resistive plate chambers. CERN-EP-2002-046, 2002. Nucl. Instr. Meth. A.

[2] Institut für Theoretische Physik, TU Graz, Graz, Austria.

[3] T. Heubrandtner, B. Schnizer, C. Lippmann, and W. Riegler. Static electric fields in an infinite plane condensor with one or three homogeneous layers. CERN-OPEN-2001-074, 2001.

[4] T. Heubrandtner, B. Schnizer, C. Lippmann, and W. Riegler. Static electric fields in an infinite plane condenser with one or three homogeneous layers. Nucl. Instr. and Meth., A 489:439-443, 2002.

[5] C. Lippmann and W. Riegler. Space charge effects and induced signals in resistive plate chambers. presented at the 'RPC 2001' - 6th Workshop on Resistive Plate Chambers and Related Detectors, 26-27 November 2001, Coimbra, Portugal. Nucl. Instr. Meth. A.

[6] C. Lippmann and W. Riegler. Detector physics of resistive plate chambers. presented at the 'IEEE NSS/MIC 2002' - Nuclear Science Symposium and Medical Imaging Conference, November 10-16, 2002, Norfolk, USA. Trans. Nucl. Sci.

[7] Discussions at the 'RPC 2001' - 6th Workshop on Resistive Plate Chambers and Related Detectors, 26-27 November 2001, Coimbra, Portugal.

[8] M. Abrescia et al. Progress in the simulation of resistive plate chambers in avalanche mode. Nucl. Instr. Meth., B (Proc. Suppl.):459-464, 1999.

[9] P. Fonte. High resolution timing of MIPs with RPCs - a model. Nucl. Instr. Meth., A 456:6-10, 2000.

[10] E. Cerron Zeballos et al. Pure avalanche mode operation of a $2 \mathrm{~mm}$ gap resistive plate chamber. Nucl. Instr. Meth., A 396:93-102, 1997. 
[11] K. Hagiwara et al. Review of particle physics. Physical Review D, 66, 2002. Available: http://pdg.lbl.gov.

[12] G. Charpak et al. Nucl. Instr. and Meth., 62:217, 1968.

[13] The LHCb collaboration. LHCb - technical proposal. CERN/LHCC-98-4 LHCC/P4, 1998. Available: http://lhcb-tp.web.cern.ch/lhcb-tp/.

[14] Talks presented about the topic 'vertex detectors in trigger'. 11th international workshop on vertex detectors, Vertex 2002, Hawaii, 2002.

[15] B. Rossi. High Energy Particles. Prentice-Hall, Inc., Englewood Cliffs, NJ, 1952.

[16] H.A. Bethe. Zur Theorie des Durchgangs schneller Korpuskularstrahlen durch Materie (theory of the passage of fast corpuscular rays through matter). Annalen der Physik, 5, 1930.

[17] H.A. Bethe. Z. Physik, 76:293, 1932.

[18] L.D. Landau. J. Exp. Phys. (USSR), 8:201, 1944.

[19] C. Grupen. Particle Detectors. Cambridge University Press, 1996.

[20] E.J. Williams. Rroc. Roy. Soc., A 169:531, 1939.

[21] H.A. Bethe. Molière's theory of multiple scattering. Phys. Rev., 89:1256-1266, 1953.

[22] W.T. Scott. The theory of small-angle multiple scattering of fast charged particles. Rev. Mod. Phys., 35:231-313, 1963.

[23] H.A. Bethe and W. Heitler. Proc. Roy. Soc., A 146:83, 1934.

[24] ALICE TRD - Transition radiation detector technical design report. CERN/LHCC/2001-021, ALICE TDR 9, 2001.

[25] HEED, program to compute the energy loss of fast particles in gases, version 1.01. CERN. Available: http://consult.cern.ch/writeup/heed/main.html.

[26] J. Keuffel. Parallel-plate counters and the measurement of very small time intervals. Phys. Rev., 73:531, 1948.

[27] J. Keuffel. Parallel plate counters. The Review of Scientific Instruments, 20(3):202-208, 1949.

[28] P. Galison. Image and Logic, A material culture of microphysics. The University of Chicago Press, Chicago, 1997. 
[29] V. Parchomchuk, Yu. Pestov, and N.V. Petrovykh. A spark counter with large area. Nucl. Instr. and Meth., 93:269-276, 1971.

[30] Yu. Pestov and G. Fedotovich. A picosecond time of flight spectrometer for the VEPP-2M based on local-discharge spark counter. IYAF-77-78-trans-E, 1978. SLAC-TRANS-184.

[31] Yu. Pestov. Timing below 100 ps with spark counters: Work principle and applications. Ric. Sci. Educ. Perm, 112:604-621, 1998. presented on the 36th International Winter Meeting on Nuclear Physics, Bormio, Italy, 1998.

[32] A. Akimov et al. Ceramic PPC technology and performance. Nucl. Instr. and Meth., A 344:120-124, 1994.

[33] ALICE TOF - Time-of-flight technical design report. CERN/LHCC 2000-012, ALICE TDR 8, 2000.

[34] G. Charpak et al. Investigation of operation of a parallel-plate avalanche chamber with a CsI photocathode under high gain conditions. Nucl. Instr. and Meth., A 307:63-68, 1991.

[35] A. Arefiev at al. Parallel plate chambers: a fast detector for ionizing particles. Nucl. Instr. and Meth., A 348:318-323, 1994.

[36] R. Santonico and R. Cardarelli. Development of resistive plate counters. Nucl. Instr. and Meth., 187:377-380, 1981.

[37] R. Santonico and R. Cardarelli. Progress in resistive plate counters. Nucl. Instr. and Meth., A 263:20-25, 1988.

[38] H. Raether. Electron Avalanches and Breakdown in Gases. Butterworths Advanced Physics Series, Washington, 1964.

[39] P. Camarri et al. Streamer suppression with SF6 in RPCs operated in avalanche mode. Nucl. Instr. and Meth., A 414:317-324, 1998.

[40] P. Fonte, V. Peskov, and B.D. Ramsey. Streamers in MSGC's and other gaseous detectors. ICFA instrumentation bulletin, Fall 1997.

[41] G. Carlino. The RPC trigger system of L3: history and current status. Scientifica Acta, 13:269-280, 1998.

[42] A. Zallo. The BABAR RPC system. Nucl. Instr. Meth., A 456:117-121, 2000.

[43] K. Abe et al. Glass RPC module for the BELLE endcap $\mathrm{K}_{l} / \mu$ detector. Scientifica Acta, 13:281-293, 1998. 
[44] C. Bacci et al. Results from the analysis of data collected with a 50m RPC carpet at yangbajing. Nucl. Instr. Meth., A 546:121-125, 2000.

[45] S. Dusini et al. Design and prototype tests of the RPC system for the OPERA spectrometers. presented at the 'RPC 2001 - 6th Workshop on Resistive Plate Chambers and Related Detectors, 26-27 November 2001, Coimbra, Portugal.

[46] G. Bencivenni et al. Performance of a test prototype for MONOLITH. Nucl. Instr. Meth., A 461:319-323, 2001.

[47] ALICE muon spectrometer technical design report. CERN/LHCC 99-22, ALICE TDR 5, page 145, 1999.

[48] Addendum to the ALICE muon spectrometer technical design report. CERN/LHCC 2000-046, Addendum 1 to ALICE TDR 5, pages 44-46, 2000.

[49] R. Arnaldi et al. Study of resistive plate chambers for the ALICE dimuon spectrometer. Nucl. Phys. B - Proc. Suppl., 78:84-89, 1999.

[50] C. Bacci et al. High altitude test of RPCs for the ARGO YBJ experiment. Nucl. Instr. Meth., A 443:342-345, 2000.

[51] Proc. II. Int. Workshop on the RPC in Particle Physics and Astrophysics, Pavia, 1993.

[52] C. Bacci et al. A hodoscope made of resistive plate chambers to identify muons in a fixed targed beauty hadroproduction experiment. Nucl. Instr. Meth., A 324:83-92, 1993.

[53] R. Cardarelli, A. Di Caiccio, and R. Santonico. Performance of a resistive plate chamber operating with pure CF3Br. Nucl. Instr. Meth., A 333:399-403, 1993.

[54] ATLAS muon spectrometer technical design report. CERN LHCC-97-22, ATLAS TDR 10, CERN, 1997.

[55] CMS muon technical design report. CMS TDR 3, CERN/LHCC 97-32, 1997.

[56] E. Cerron Zeballos et al. A new type of resistive plate chamber: the multigap RPC. Nucl. Instr. Meth., A 374:132-136, 1996.

[57] P. Fonte, A. Smirnitski, and M.C.S. Williams. A new high resolution TOF technology. Nucl. Instr. Meth., A 443:201-204, 2000.

[58] M. Bogomilov et al. The RPC time-of-flight system of the HARP experiment. presented at the 'RPC 2001' - 6th Workshop on Resistive Plate Chambers and Related Detectors, 26-27 November 2001, Coimbra, Portugal. 
[59] E. Keil. The large hadron collider LHC. CERN-LHC-PROJECT-REPORT-079, 1996. Talk given at the 1996 DPF / DPB Summer Study on New Directions for High-Energy Physics (SNOWMASS 96), Snowmass, CO, USA, 25.June 12.July 1996.

[60] The large hadron collider (LHC) project. Available: http://hc-newhomepage.web.cern.ch/lhc-new-homepage.

[61] The ATLAS collaboration. ATLAS - technical proposal for a general-purpose pp experiment at the large hadron collider at cern. CERN/LHCC-94-43 LHCC/P2, 1994. Available: http://atlas.web.cern.ch/Atlas/TP/tp.html.

[62] The CMS collaboration. CMS - technical proposal. CERN-LHCC-94-38 LHCC/P1, 1994.

[63] The ALICE collaboration. A large ion collider experiment - technical proposal. CERN/LHCC-95-71 LHCC/P3, 1995. Available: http://consult.cern.ch/alice/Documents/1995/01/abstract.

[64] A. Akindinov et al. The multigap resistive plate chamber as a time-of-flight detector. Nucl. Instr. Meth., A 456:16-22, 2000.

[65] ALICE TPC - Time projection chamber technical design report. CERN/LHCC 2000-001, ALICE TDR 7, 2000.

[66] Addendum to the ALICE TOF - Time-of-flight technical design report. CERN/LHCC 2002-016, 2002.

[67] ALICE HMPID - High momentum particle identification technical design report. CERN/LHCC/1998-019, ALICE TDR 1, 2001.

[68] The HARP collaboration. Proposal for an RPC TOF system. 2000. Available: http://harp.web.cern.ch/harp.

[69] P. Fonte et al. High resolution RPCs for large TOF systems. Nucl. Instr. and Meth., A 449:295-301, 2000.

[70] M.C.S. Williams et al. The multigap RPC : The time-of-flight detector for the ALICE experiment. Nucl. Instr. Meth., A 478:183-186, 2002.

[71] M. Abrescia et al. The simulation of resistive plate chambers in avalanche mode: Charge spectra and efficiency. Nucl. Instr. Meth., A 431:413-427, 1999.

[72] G. Aielli. Advanced studies on RPCs. Dissertation, Università Degli Studi Di Roma "Tor Vergata", December 2000. 
[73] G. Aielli. Logistic saturated avalance model. presented at the 'RPC 2001' - 6th Workshop on Resistive Plate Chambers and Related Detectors, 26-27 November 2001, Coimbra, Portugal.

[74] F. Sauli. Principles of operation of multiwire proportional and drift chambers. CERN Yellow Report 77-09, 1977.

[75] E.F. Rieke and W. Prepejchal. Ionisation cross-sections of gaseous atoms and molecules for high-energy electrons and positrons. Phys. Rev., A 6:1507, 1972.

[76] S. Biagi, MAGBOLTZ, program to compute gas transport parameters, version 2.2. CERN. Available: http://consult.cern.ch/writeup/magboltz/.

[77] S. Biagi, IMONTE, program to compute gas transport parameters. CERN.

[78] W.W.M. Allison and J.H. Cobb. Relativistic charged particle identification by energy loss. Ann. Rev. Nucl. Part. Sci., 30:253-298, 1980.

[79] HEED example data for minimum ionizing particles, see reference [25].

[80] F. Lapique and F. Piuz. Simulation of the measurement by primary cluster counting of the energy lost by a relativistic ionizing particle in argon. Nucl. Instr. and Meth., 175:297-318, 1980.

[81] Eduardo Gorini. Measurements of drift velocity and amplification coefficient in $\mathrm{C}_{2} \mathrm{H}_{2} \mathrm{~F}_{4}$-isobutane mixtures for avalanche operated RPC. presented at the 'RPC 1997' - 4th Workshop on Resistive Plate Chambers and Related Detectors, October 1997, Napoli, Italy.

[82] V. Golovatyuk. Laser beam studies of RPC behaviour in avalanche mode. presented at the 'RPC 2001' - 6th Workshop on Resistive Plate Chambers and Related Detectors, 26-27 November 2001, Coimbra, Portugal.

[83] G. Schultz. Thesis, Univerity of Strassbourg, 1976.

[84] P. Ramanantsizehena. Thesis, Univerity of Strassbourg, 1979. CRN-HE79-13.

[85] W. Legler. Die Statistik der Elektronenlawinen in elektronegativen Gasen, bei hohen Feldstärken und bei hoher Gasverstärkung (the statistics of electron avalanches in electronegative gases at high electric field strengths and at large gas gain). Z. Naturforschung, 16a:253-261, 1961.

[86] S. Ramo. Currents induced in electron motion. PROC. IRE 27, page 584, 1939.

[87] W. Riegler. Induced signals in resistive plate chambers. Nucl. Instr. and Meth., A 491:258-271, 2002. 
[88] P. Fonte. Applications and new developments in resistive plate chambers. ICFA instrumentation bulletin, 23, Fall 2001.

[89] I. Kitayama et al. Optical observation of discharge in resistive plate chamber. Nucl. Instr. Meth., A 424:474-482, 1999.

[90] A. Semak et al. Properties of discharge in the narrow gap glass RPC. Nucl. Instr. Meth., A 456:50-54, 2000.

[91] R. Cardarelli, R. Santonico, and V. Makeev. The avalanche to streamer transition in RPCs. presented at the 'RPC 1995' - 3rd Workshop on Resistive Plate Chambers and Related Detectors, October 1995, Pavia, Italy.

[92] R. Cardarelli, R. Santonico, and V. Makeev. Avalanche and streamer mode operation of resistive plate chambers. Nucl. Instr. Meth., A 382:470-474, 1996.

[93] P. Fonte. A model of breakdown in parallel-plate chambers. IEEE Trans. Nucl. Science, 43:2135-2140, 1996.

[94] GARFIELD, simulation of gaseous detectors, version 7.04. CERN, 2001. Available: http://consult.cern.ch/writeup/garfield.

[95] ROOT, an object-oriented data analysis framework, version 3.02. CERN, 2001. Available: http://root.cern.ch.

[96] P. Fonte and V. Peskov. High-resolution TOF with RPCs. preprint LIP/00-04, 2000.

[97] A. Akindinov et al. A four-gap glass-RPC time-of-flight array with 90ps time resolution. IEEE Trans. Nucl. Sci., 48(5):1658-1663, 2001. also a CERN note: CERN-EP 99-166.

[98] Mathematica 4.2. Wolfram Research, $2003 . \quad$ Available: http://www.woltram.com.

[99] P. Fonte, Development of large area and of position sensitive timing RPCs. Presented at the 9th Vienna Conference on Instrumentation, Vienna, Austria, February 19-23, 2001.

[100] G. Aielli et al. Performance of a large-size RPC equipped with the final ATLAS front end electronics at X5-GIF irradiation facility. Nucl. Instr. Meth., A 456:77$81,2000$.

[101] A. Blanco et al. A new front-end electronics chain for timing RPCs. IEEE Trans. Nucl. Science, 48(4), 2001. 
[102] P. Fonte. Resistive plate chambers for time of flight. Talk presented at the 'Compressed Baryonic Matter Workshop', May 13-16, 2002, GSI Darmstadt, Germany.

[103] P. Fonte, private communication.

[104] I.N. Bronstein and K.A. Semedjajew. Taschenbuch der Mathematik. Verlag Nauka, Moscow and BSB B. G. Teubner Verlagsgesellschaft, Leipzig, 1985.

[105] W. Blum and L. Rolandi. Particle Detection with Drift Chambers. Springer Verlag, 1993.

[106] M. Atac et al. Self-quenching streamers. Nucl. Instrum. Meth., 200:345, 1982.

[107] G. Aielli et al. RPC ageing studies. Nucl. Instrum. Meth., A 478:271-276, 2002.

[108] C. Moeller. Annalen der Physik, 14:531, 1932.

[109] H.J. Bhabha. Proc. Roy. Soc., A 154:195, 1936.

[110] H.J. Bhabha. Proc. Roy. Soc., A 164:257, 1938.

[111] H.J. Massey and H.C. Corben. Proc. Camb. Phil. Soc., 35:463, 1939.

[112] J.R. Oppenheimer, H. Snyder, and R. Serber. Phys. Rev., 57:75, 1940. 


\section{Danksagung}

Während der Dissertation war ich als "doctoral student” am Europäischen Kernforschungszentrum (CERN) in Genf angestellt. Mein allererster Dank gilt meinem Doktorvater Prof. Dr. Reinhard Stock dafür, daß er die Betreuung der Arbeit übernommen hat. Ein ganz besonderer Dank gilt Dr. Werner Riegler für die intensive Betreuung vor Ort am CERN und die vielen fruchtbaren und kritischen Diskussionen. Ich habe sehr viel gelernt. Ausßerdem danke ich Dr. Paulo Fonte für die interessanten und anregenden Diskussionen in Coimbra und per Email. Natürlich gilt mein Dank auch allen Mitarbeitern der CERN-Gruppe der LHCb-Kollaboration, in die ich integriert war, insbesondere Dr. Burkhard Schmidt und Thomas Schneider. Und schließlich danke ich noch Prof. Dr. Herbert Ströbele für die Bereitschaft, die Arbeit zu begutachten.

Genf, April 2002

Christian Lippmann 


\section{Curriculum Vitae}

$\begin{array}{lll}\text { Persönliche Informationen } & \text { Name: } & \text { Christian Lippmann } \\ & \text { Familienstand: } & \text { ledig } \\ & \text { Staatsangehörigkeit: } & \text { deutsch } \\ & \text { Geburtsdatum: } & 22.02 .1974 \\ \text { Geburtsort: } & \text { Kronberg/Ts. }\end{array}$

\begin{tabular}{|c|c|c|}
\hline Schulbildung & $1980-1984$ & Besuch der Grundschule Glashütten/Ts. \\
\hline & 1984-1986 & $\begin{array}{l}\text { Besuch der Förderstufe der Taunusschule König- } \\
\text { stein/Ts. }\end{array}$ \\
\hline & $1986-1993$ & $\begin{array}{l}\text { Besuch des Gymnasiums der Taunusschule Kö- } \\
\text { nigstein/Ts. }\end{array}$ \\
\hline & & Abschluss: Abitur \\
\hline Zivildienst & $1993-1994$ & $\begin{array}{l}\text { Mobiler Sozialer Dienst, Caritas Sozialstation, } \\
\text { Königstein/Ts. }\end{array}$ \\
\hline Studium & $1996-2000$ & $\begin{array}{l}\text { Studium der Physik an der Johann-Wolfgang- } \\
\text { Goethe Universität Frankfurt }\end{array}$ \\
\hline & 1997 & Vordiplom, Note: "Sehr Gut" \\
\hline & $1999-2000$ & $\begin{array}{l}\text { Diplomarbeit an der Gesellschaft für Schwerionen- } \\
\text { forschung } \mathrm{mbH}(\mathrm{GSI}) \text { in Darmstadt }\end{array}$ \\
\hline & & Titel: "Aufbau und Inbetriebnahme eines Gasquali- \\
\hline & & tätsmonitors für die HADES-Driftkammern” \\
\hline & & Betreuer: Prof. Dr. Herbert Ströbele, Dr. Joachim \\
\hline & & Stroth \\
\hline & 2000 & Diplom im Fach Physik, Note: "Sehr Gut" \\
\hline & 2001 & Beginn der Promotion als "doctoral student" am \\
\hline & & Europäischen Kernforschungszentrum (CERN) in \\
\hline & & Genf. Betreuer: Prof. Dr. Reinhard Stock und \\
\hline & & Dr. Werner Riegler am CERN \\
\hline
\end{tabular}

Sonstiges 1995-1996 Einjährige Weltreise 